Florida International University FIU Digital Commons

$11-4-2016$

\title{
Identification of the Active Odors From Illicit Substances for the Development of Optimal Canine Training Aids
}

Adhly M. Huertas-Rivera

ahuer005@fiu.edu

DOI: 10.25148 /etd.FIDC001232

Follow this and additional works at: https://digitalcommons.fiu.edu/etd

\section{Recommended Citation}

Huertas-Rivera, Adhly M., "Identification of the Active Odors From Illicit Substances for the Development of Optimal Canine Training Aids" (2016). FIU Electronic Theses and Dissertations. 2990.

https://digitalcommons.fiu.edu/etd/2990 


\title{
FLORIDA INTERNATIONAL UNIVERSITY
}

Miami, Florida

\section{IDENTIFICATION OF THE ACTIVE ODORS FROM ILLICIT SUBSTANCES}

FOR THE DEVELOPMENT OF OPTIMAL CANINE TRAINING AIDS

\author{
A dissertation submitted in partial fulfillment of \\ the requirements for the degree of \\ DOCTOR OF PHILOSOPHY \\ in \\ CHEMISTRY \\ by
}

Adhly Marie Huertas Rivera

2016 
To: Dean Michael R. Heithaus

College of Arts, Sciences and Education

This dissertation, written by Adhly Marie Huertas Rivera, and entitled Identification of the Active Odors from Illicit Substances for the Development of Optimal Canine Training Aids, having been approved in respect to style and intellectual content, is referred to you for judgment.

We have read this dissertation and recommend that it be approved.

$\begin{array}{r}\hline \text { Yong Cai } \\ \hline \text { Stewart D'Alessio } \\ \hline \text { Anthony DeCaprio } \\ \hline \text { Jeffrey Joens } \\ \hline \text { Kenneth Furton, Major Professor }\end{array}$

Date of Defense: November 4, 2016

The dissertation of Adhly Marie Huertas Rivera is approved.

Dean Michael R. Heithaus College of Arts, Sciences and Education

Andrés G. Gil

Vice President for Research and Economic Development and Dean of the University Graduate School

Florida International University, 2016 
(C) Copyright 2016 by Adhly Marie Huertas Rivera All rights reserved. 


\section{DEDICATION}

To my EVERYTHING, my family. 


\section{ACKNOWLEDGMENTS}

First and foremost, Dr. Furton, although I started working in the lab short after you were promoted to Dean, you accepted me in the group with no hesitation. I could not imagine myself working in another group but yours. Your passion to forensics and interesting research drove me straight to you. Even though it has been a rough and long path, if I were given the chance to choose over again I wouldn't have changed my decision. The amazing students you have chosen over the years, made this journey a lot better.

The members of my dissertation committee, Yong Cai, Anthony DeCaprio, Stewart D'Alessio, and Jeff Joens, have generously given their time and expertise to better my work. Howard, you have always been there regardless if it is your duty or not, and for that I will forever appreciate you. Thank you for your contribution and your good-natured support. Jessie, for almost three years the $15^{\text {th }}$ of each month was not my favorite day, but I have to admit that is because of you that I am stronger that when I first came. Thank you, because out of this project we built a friendship that I hope never ends. Atilda, and Maggie, I know I have bother you both A LOT, but I do it just because I know you are the best guidance I can have. Thank you for always trying your best, to either answer my questions, or for helping me go above and beyond to complete my paperwork and/or requirements.

I want to thank Peter (Asere) Nuñez and his tribe. Since day one you demonstrated how satisfying is for you to help others, and you definitely did. You gave me all the essentials I needed to make out of this research something valuable, not only you provided me with what you had available at the moment, but without thinking about it twice, you just made a few calls and made possible I had everything needed to complete this project, 
quite simply, I wouldn't have been able to complete all this work without your help and assistance.

Furtonians, you guys are unique and exceptional. Honestly, there are not enough words to describe what you all mean to me. Your unconditional support is unmeasurable; your willingness to help each other is the best tool a team can ask for, and that's exactly what make you all the best. Despite our differences, we always were available for each other and in no time, we were not only lab mates but a diverse family.

My Miami family, Salcedo-Santos y Meza-Salcedo, oh boy, there is nothing more difficult than to leave home to follow your dreams, and there is nothing better than to feel home in a house full of love, caring and fun. Yashi, with no hesitation you open your family circle to me, and now not only I feel part of your family but I'm the luckiest girl for having Amber as my beautiful goddaughter. I hope I can be the best example for her, and to take care of her if at any point you are absent. Thank you for making your friends, my friends, and for your love and support through this long journey, and forever.

Emmanuel, you walked into this craziness I call life halfway through my Ph.D. but you are still a hero for keeping up with me. My stress, mood swings, and days of crying have never (that I know of) make you run away, and for that, I thank you too.

Last but not least, my eternal cheerleaders; my family. Mami, Papi y Maran even after having a bad day when I didn't want to talk, you three still gave me the strength and unconditional support to keep up going regardless of the stones that were on my way. There are not enough words to describe how much your support means when you are 1,014 miles away (yes, I looked it up). 
This project was partially supported by TSWG Task SC-3388C, awarded by the Technical Support Working Group. This project was partially funded through financial support of a Florida International University College of Arts, Sciences \& Education Doctoral Evidence Acquisition Fellowship. 


\title{
ABSTRACT OF THE DISSERTATION \\ IDENTIFICATION OF THE ACTIVE ODORS FROM ILLICIT SUBSTANCES \\ FOR THE DEVELOPMENT OF OPTIMAL CANINE TRAINING AIDS
}

\author{
by \\ Adhly Marie Huertas Rivera \\ Florida International University, 2016 \\ Miami, Florida \\ Professor Kenneth Furton, Major Professor
}

The exploitation of illicit substances, such as drugs and explosives, is on the rise. Special attention must therefore be considered to reduce the transportation and storage of these illicit substances by improving the capability of detection, even when hidden from view. Although analytical methods of detection for both drugs and explosives have improved over time, biological detectors, such as canines, are still commonly used. In comparison to humans, these canines have a larger number of olfactory receptors and a greater olfactory epithelium surface area, providing them with a more enhanced olfaction than that of humans.

The premise for the detection of illicit drugs and explosives is based on the premise that these substances though hidden, will emit volatile organic compounds (VOCs). These VOCs are not often the parent drug or explosive, they are essentially a chemical associated with the source and provide a reliable indication of the illicit substance. Previous successful research has been conducted on the identification of the active odors present in the headspace of cocaine, methamphetamine, and MDMA but instead for marijuana and heroin there have been minimum success. Thus, in the present research a method using headspace 
solid-phase microextraction coupled to gas chromatography-mass spectrometry (HSSPME-GC-MS) was optimized to identify the VOCs makeup of heroin and marijuana to further identify the active odor compound(s) responsible for the alert response of biological detectors (canines). A mixture of acetic acid and acetylsalicylic acid was identified as target odor mimic for heroin by certified detector canines, while a mixture of limonene and caryophyllene was recognized as odor mimic for marijuana by conducting ORTs. The training aids developed successfully mimic the scent of the actual illicit substance and can be used to improve the capabilities of both drug and explosive detection canines.

Additionally, as growing threat of improvised explosives has created a worldwide concern and emphasized the requirement of a greater spectra of canine training aids that covers the complete range of explosives available, a new approach for the creation of training aids for IEDs have been evaluated. The use of a dynamic collection system have proved to be an option to develop fast and reliable canine training aids for IEDs. 


\section{TABLE OF CONTENTS}

CHAPTER

PAGE

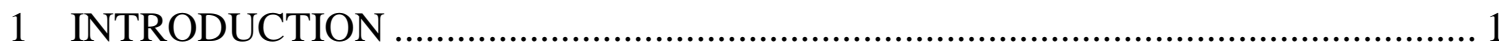

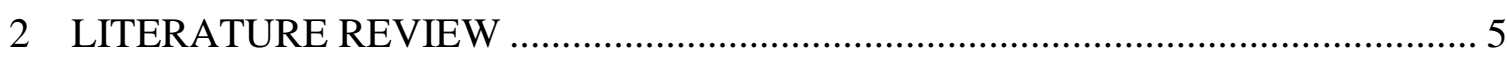

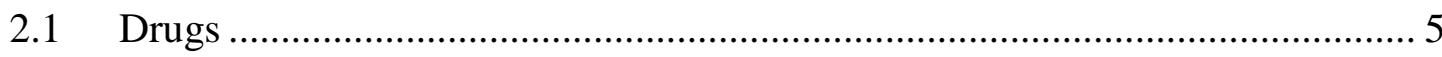

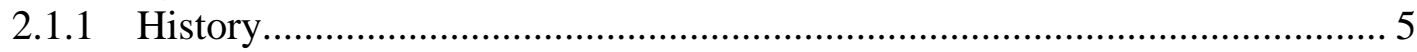

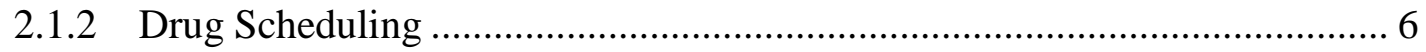

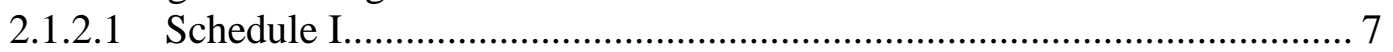

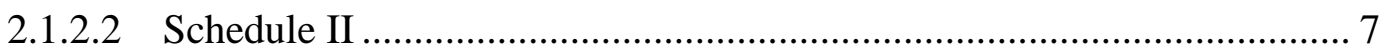

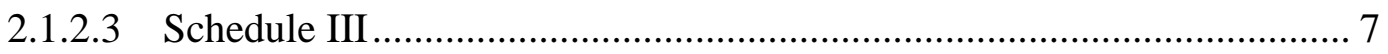

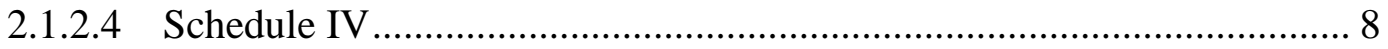

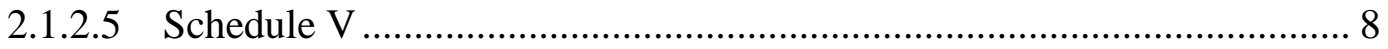

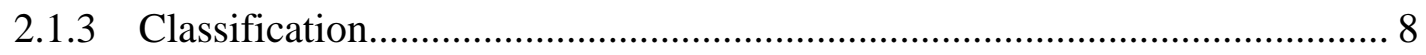

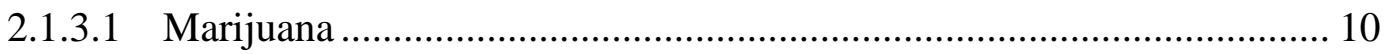

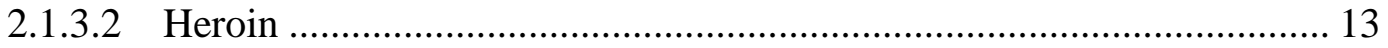

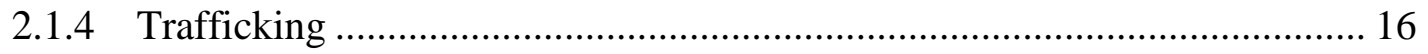

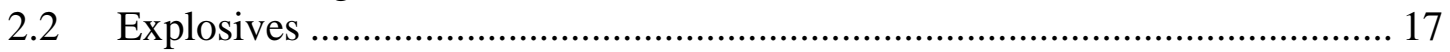

2.2.1 History and classification........................................................................ 18

2.2.2 Triacetone Triperoxide (TATP) (56) ………………............................. 20

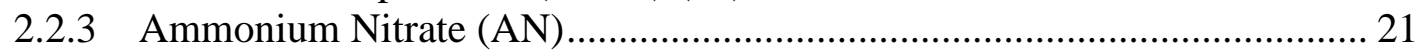

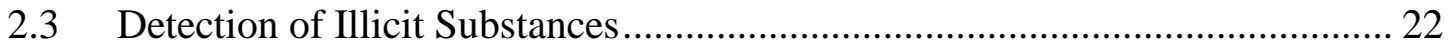

2.3.1 Sampling Techniques........................................................................ 23

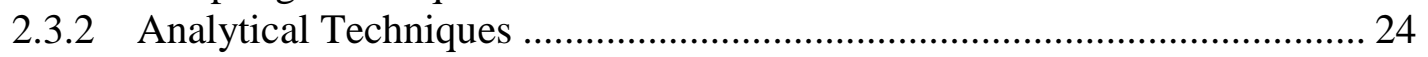

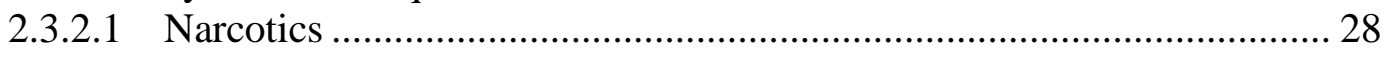

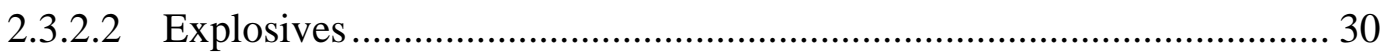

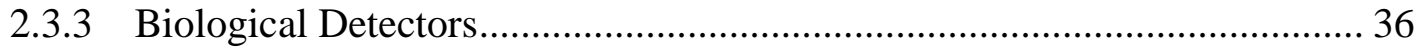

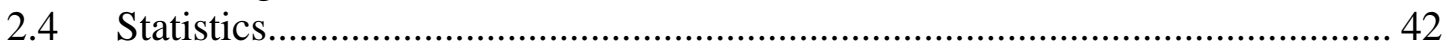

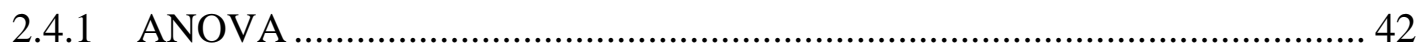

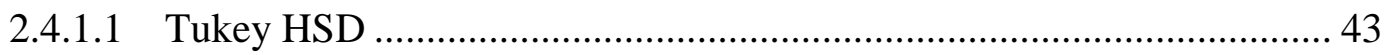

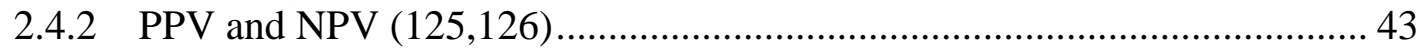

2.4.2.1 Positive Predictive Value (PPV) ....................................................... 43

2.4.2.2 Negative Predictive Value (NPV) ......................................................... 44

3 SIGNIFICANCE OF STUDY AND RESEARCH OBJECTIVES ………………...... 44

4 TASK 1. OPTIMIZATION OF SAMPLING AND ANALYSIS METHOD ............. 47

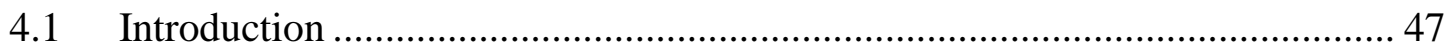

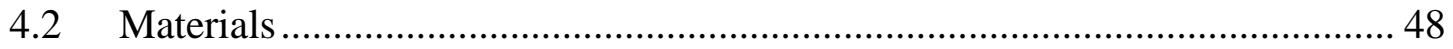

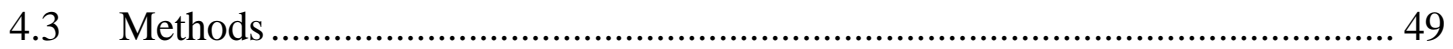

4.3.1 Extraction Method ............................................................................... 49

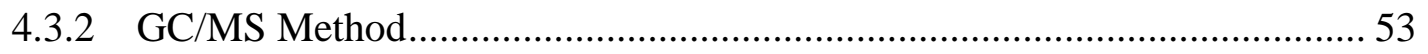

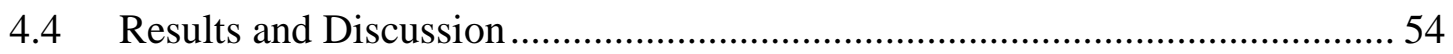

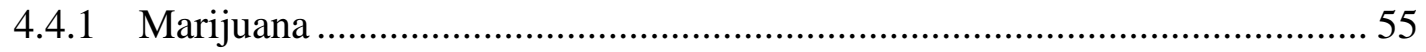




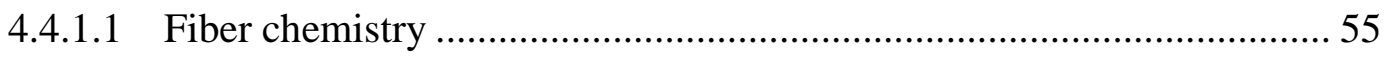

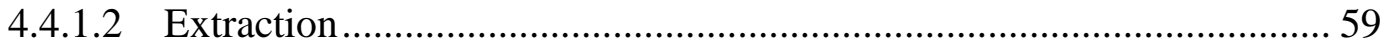

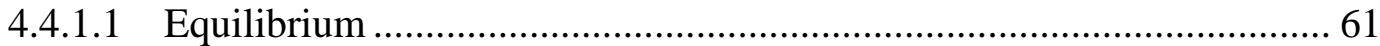

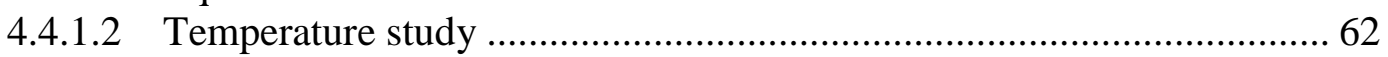

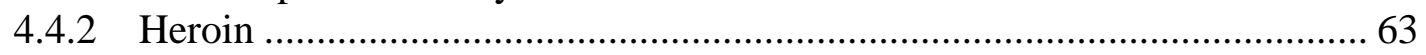

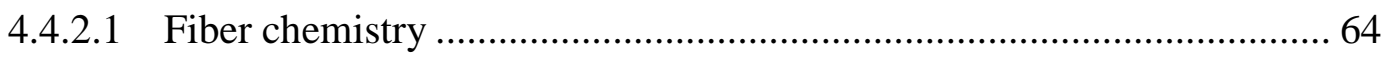

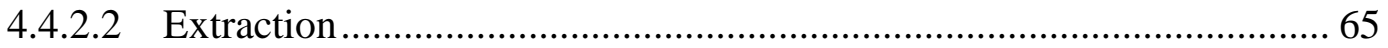

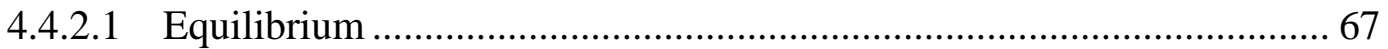

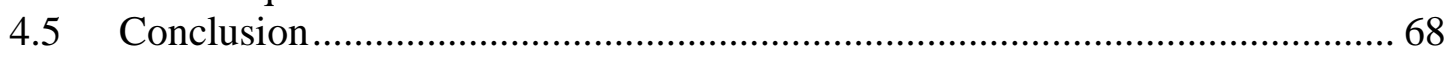

5 TASK 2. DETERMINATION OF VOCs FROM ILLICIT SUBSTANCES ............. 70

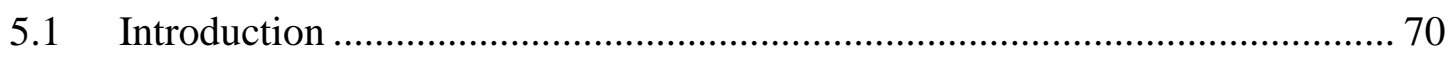

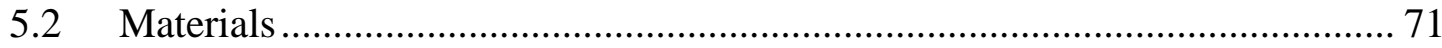

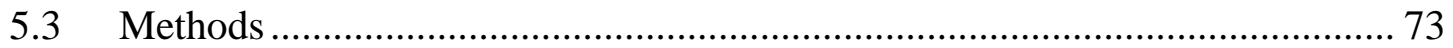

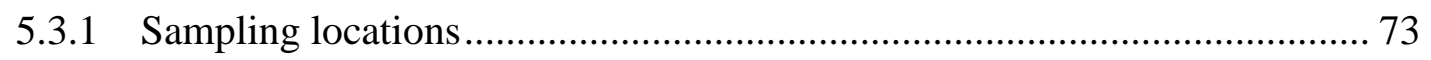

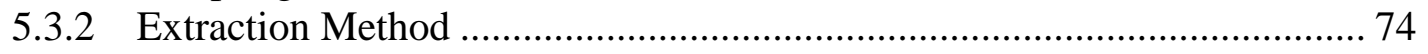

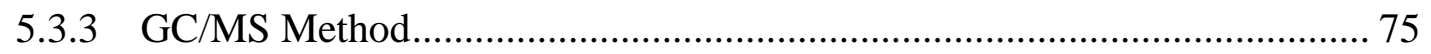

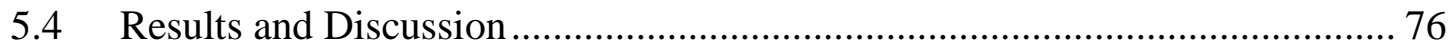

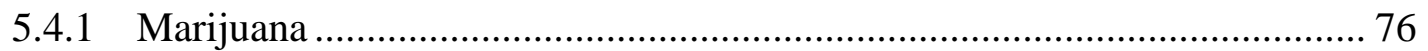

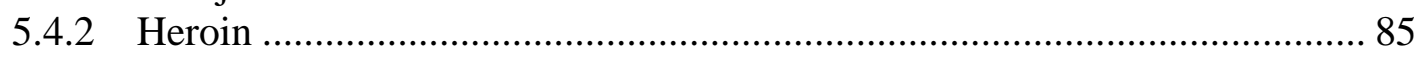

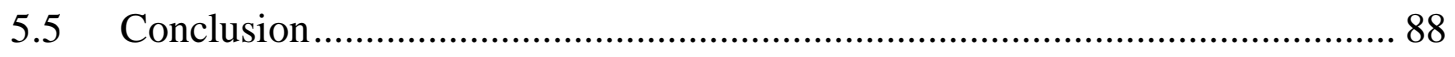

6 TASK 3. ACTIVE ODOR DETERMINATION USING BIOLOGICAL

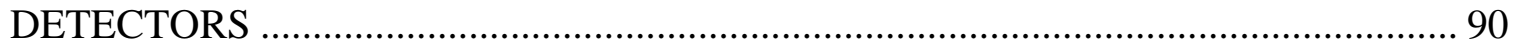

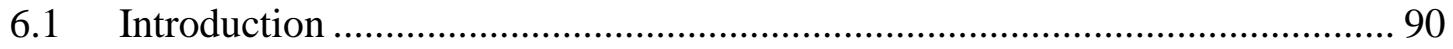

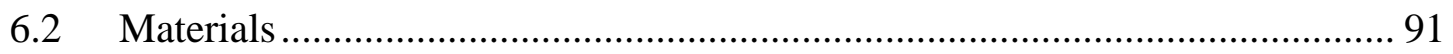

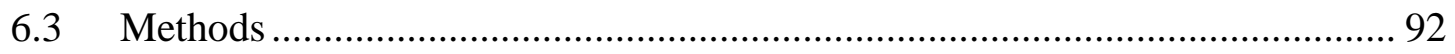

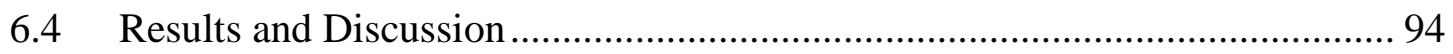

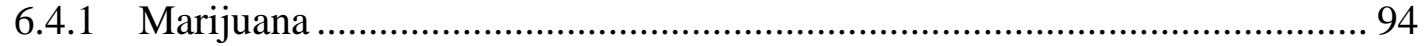

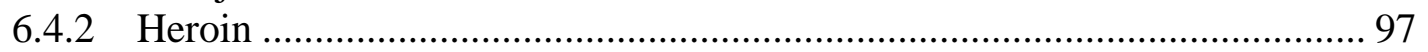

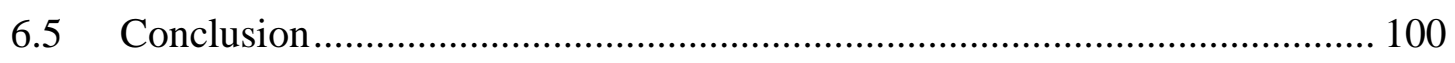

7 TASK 4. FORMATION OF CONTROLLED ODOR MIMIC PERMEATION

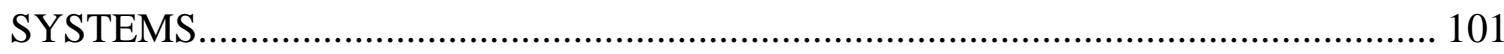

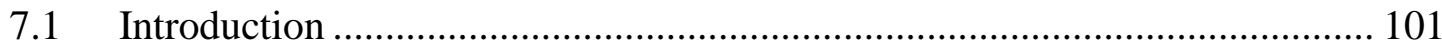

7.2 Materials and Method.......................................................................... 102

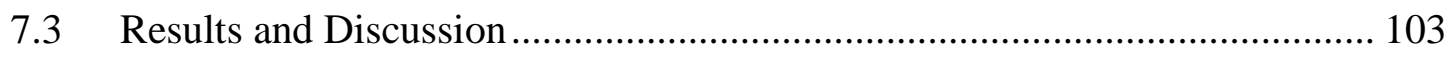

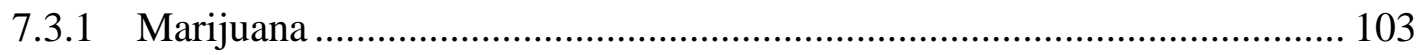

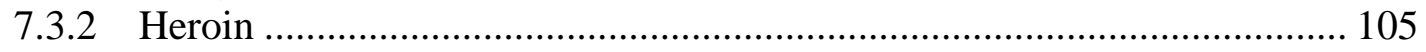

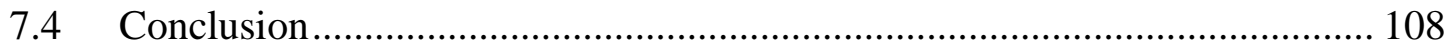

8 TASK 5. FIELD TRIAL VALIDATION OF NEWLY DEVELOPED TRAINING

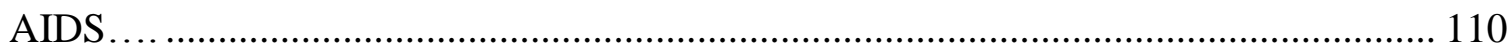

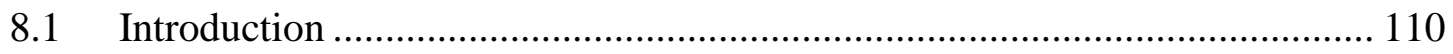

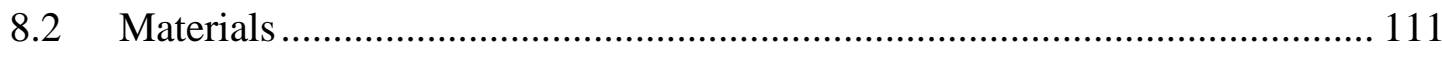

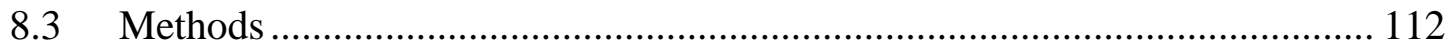




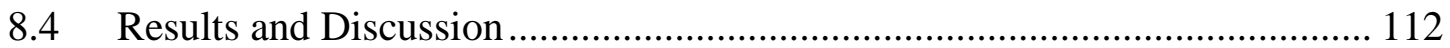

8.4.1 Marijuana ......................................................................................... 112

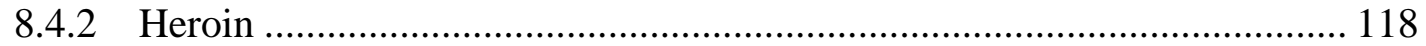

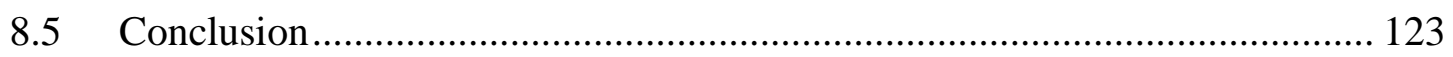

9 TASK 6. TRAINING AIDS DEVELOPMENT ALTERNATIVE FOR NEWLY

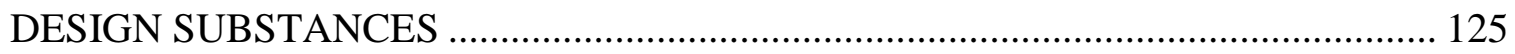

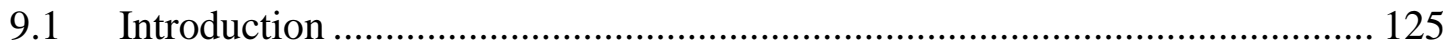

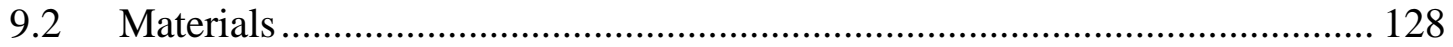

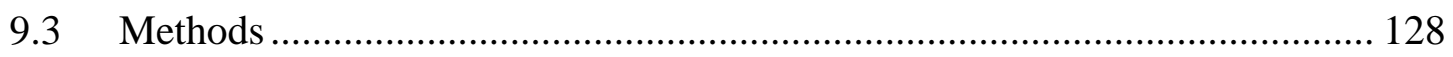

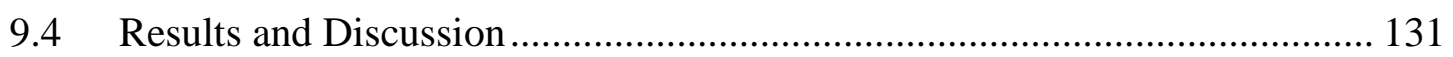

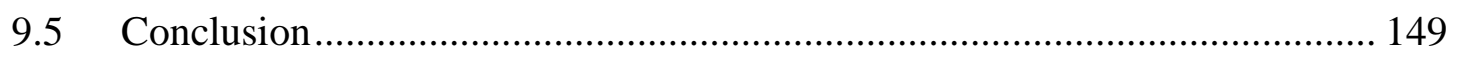

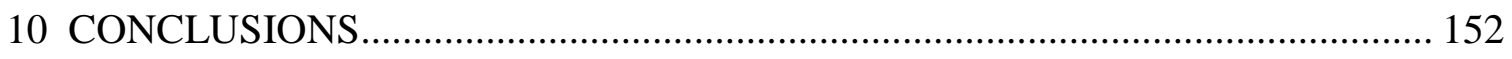

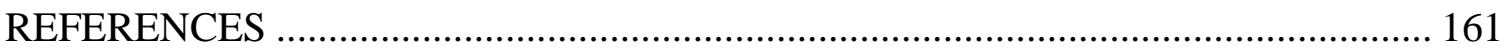

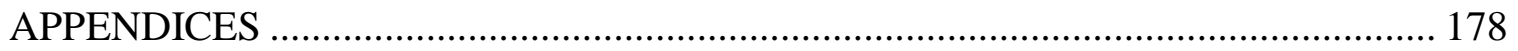

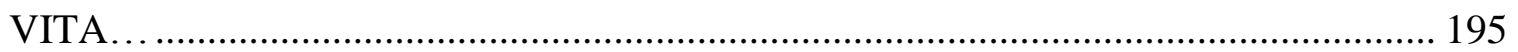




\section{LIST OF TABLES}

TABLE

PAGE

Table 1. SPME Fiber Selection.......................................................................... 48

Table 2. Temperature and Conditioning Recommendations for GC .............................. 50

Table 3. Percentage of Total Peak Area of Each Chemical Family ............................... 59

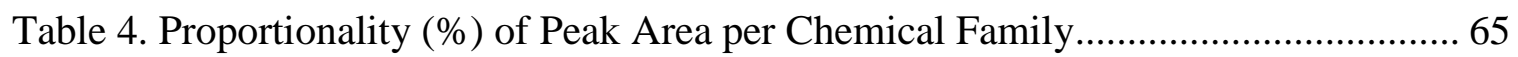

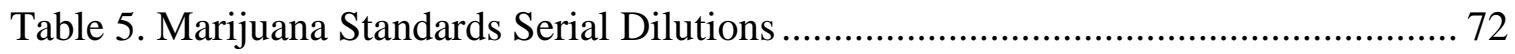

Table 6. Heroin Standards Serial Dilutions ............................................................ 73

Table 7. Optimized Parameters for SPME Extraction ............................................. 74

Table 8. Composition of the Volatiles in Marijuana Samples ....................................... 77

Table 9. Contribution (\%) of Compounds in Marijuana Headspace ............................. 82

Table 10. Certified Canines: Breed, Age and Gender Data ........................................... 92

Table 11. Individual Canine Performance Table on Potential Pseudo Marijuana

Training Aid (Numerator $=$ A, Denominator $=$ No alert, Parenthesis $=$ Interest) $\ldots \ldots \ldots \ldots \ldots . . . . . . .95$

Table 12. Odor Recognition Test (Average): Single-Compound Pseudo Marijuana

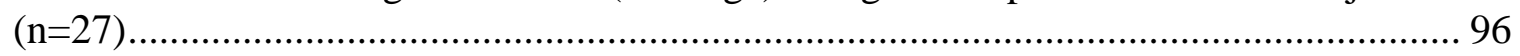

Table 13. Individual Canine Performance Table on Potential Multi-compound Pseudo Marijuana (Numerator=A, Denominator=No alert, Parenthesis= Interest) $\ldots \ldots \ldots \ldots \ldots \ldots \ldots . . . . . . . .97$

Table 14. Individual canine performance table on potential pseudo heroin training aid

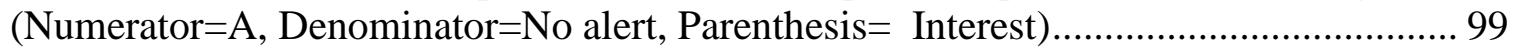

Table 15. Odor Recognition Test (Average): Pseudo Heroin $(\mathrm{n}=32)$........................... 99

Table 16. Individual Canine Performance Table on Pseudo Marijuana Training Aid

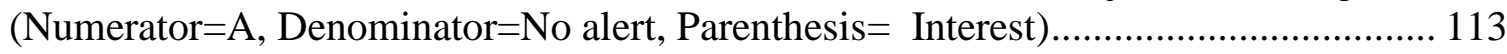

Table 17. Odor Recognition Test (Average): Pseudo Marijuana (n=6)........................ 114

Table 18. Individual Canine Performance Table on 1 g Comestible Products $(n=5)$

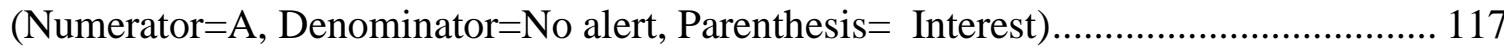

Table 19. Odor Recognition Test (Average): Comestible Products $(\mathrm{n}=5)$.................... 117 
Table 20. Individual Canine Performance Table on Pseudo Heroin Training Aid $($ Numerator $=$ A, Denominator $=$ No alert, Parenthesis $=$ Interest $)$. 118

Table 21. Odor Recognition Test (Average): Pseudo Heroin $(n=12)$. 119

Table 22. Individual Canine Performance Table on Commercially and Comestible Products $(\mathrm{n}=5)($ Numerator $=$ A, Denominator $=$ No alert, Parenthesis= Interest $) \ldots \ldots \ldots \ldots . . . .122$

Table 23. Odor Recognition Test: Commercial and Comestible Products $(n=5) \ldots \ldots \ldots . .123$

Table 24. Target Compound per Explosive Family ................................................. 127

Table 25. GC-MS Methods for Target Compounds Detection .................................... 130

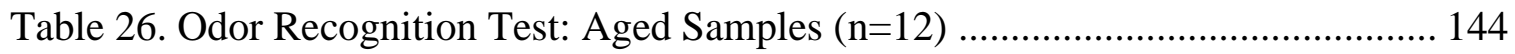

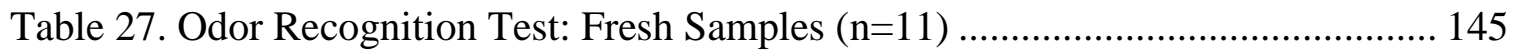

Table 28. Suitable Parameters for the Dynamic Collection of VOCs ......................... 151

Table 29. Chemical Product Physical and Chemical Properties ................................. 178

Table 30. Canine rooster and trials schedule (Marijuana) ........................................ 179

Table 31. Canine ORTs Single Compound Raw Data (Marijuana n=27) .................... 181

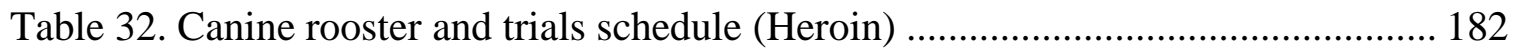

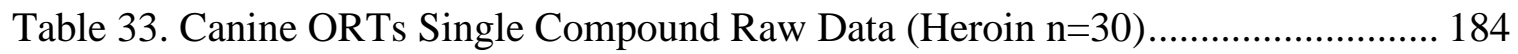

Table 34. Canine rooster and trials schedule (Pseudo Heroin) .................................... 185

Table 35. Canine ORTs Pseudo Heroin Raw Data $(\mathrm{n}=12)$......................................... 187

Table 36. Canine rooster and trial schedule ........................................................... 193

Table 37. Canine ORTs Water Gel Aged Dynamic Collected Samples $(n=12)$............ 194

Table 38. Canine ORTs Water Gel Fresh Dynamic Collected Samples ( $\mathrm{n}=12) \ldots \ldots \ldots . . . . .194$ 


\section{LIST OF FIGURES}

FIGURE

PAGE

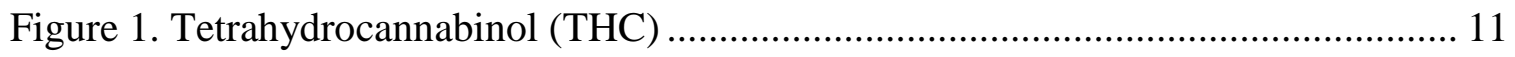

Figure 2. THC binding to cannabinoid receptor (CB1) (30) ....................................... 12

Figure 3. Chemical Synthesis of Heroin (a) Morphine (b) Acetic anhydride (c) Diacetylmorphine (36) .......................................................................................... 14

Figure 4. Opiates binding to opiate receptors (30) ……………………...................... 15

Figure 5. Classification of Explosives ……………………...................................... 19

Figure 6. Triacetone Triperoxide (56) Molecule ......................................................... 20

Figure 7. Ammonium Nitrate (AN) …………………………………………..... 22

Figure 8. SPME Fiber and Holder Assembly ………………..................................... 25

Figure 9. Gas Chromatography- Mass Spectrometer Scheme ………………………...... 32

Figure 10. Mass Spectrum for Limonene A. Base Peak, B. Parent Peak ……….............. 35

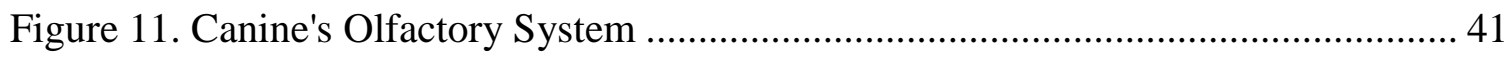

Figure 12. Triplicate of Heroin Samples and Blank Extraction Using SPME.................. 52

Figure 13. Abundance and No. of VOCs Found in Marijuana Headspace Samples Using Different Fiber Chemistries.............................................................................. 57

Figure 14. Abundance of 15, 30, 45, 60, and 90 Extraction Time of $0.5 \mathrm{~g}$ of Marijuana

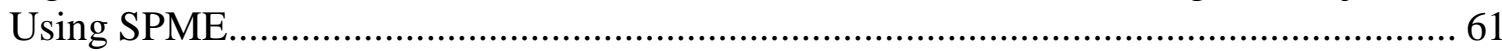

Figure 15. Abundance of 15, 30, 45 and 60 Equilibrium Time of $0.50 \mathrm{~g}$ of Marijuana

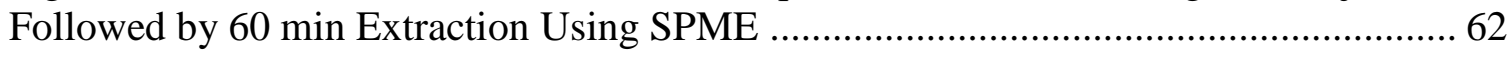

Figure 16. Abundance and No. of Compounds of $0.5 \mathrm{~g}$ Marijuana Samples Extracted

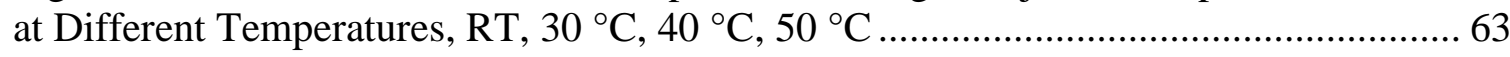

Figure 17. Abundance of a 15, 30, 45, 90 min SPME Equilibrium of $0.50 \mathrm{~g}$ of Heroin

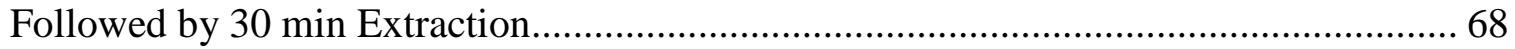

Figure 18. Marijuana HS-SPME Chromatogram (DVB/CAR/PDMS: 15 min Equilibrium, 45 min Extraction) .............................................................................. 79

Figure 19. Compounds Frequency of Occurrence (\%) in Marijuana Samples ( $\mathrm{n}=10)$..... 80 
Figure 20. Relative Abundance of Compounds in Marijuana Samples . 80

Figure 21. Calibration curves for Marijuana Most Frequent Headspace Compounds ..... 83

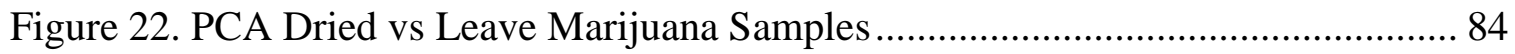

Figure 23. Heroin HS-SPME Chromatogram (DVB/CAR/PDMS: 45 min Equilibrium,

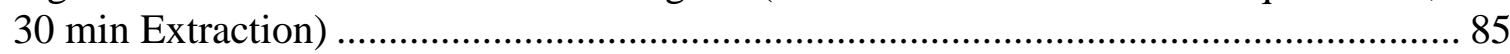

Figure 24. Compounds Frequency of Occurrence $(\%)$ in Heroin Samples $(n=5) \ldots \ldots \ldots . . .86$

Figure 25. Relative Abundance of Compounds in the Headspace of Heroin Samples..... 87

Figure 26. Triplicate of the Headspace of "Black Tar" Heroin Samples .......................... 88

Figure 27. Methodology for Dissipation Rate Study ............................................... 103

Figure 28. Cellulose based training aid mimic for marijuana................................... 104

Figure 29. Alumina Based Training Aid Mimic for Marijuana ................................... 105

Figure 30. (A) Hexanal $10 \mathrm{mmHg} @ 20{ }^{\circ} \mathrm{C}$ (B) Acetic Acid $11.4 \mathrm{mmHg} @ 20{ }^{\circ} \mathrm{C} . . . . .106$

Figure 31. Assymetrical ESIS (Hexanal)-Acetic Acid ............................................ 107

Figure 32. Pseudo Heroin Dissipation Over Time ...................................................... 108

Figure 33. Headspace of Marijuana and Different Comestible Products Containing Limonene (Red) and/or Caryophyllene (Purple) as a Main Chemical Compound......... 116

Figure 34. Headspace of Heroin and Different Commercially Available Products Containing Acetic Acid (Red) as a Main Chemical Compound...

Figure 35. (A). Optimized parameters are selected using the drop down menu on the screen (B). Gauze pad is inserted and clipped in the instrument (C). The instrument is positioned upside down facing the sample for collection ...................................... 130

Figure 36. Ammonium Nitrate Derivatization Product Headspace Using SPME-GC-

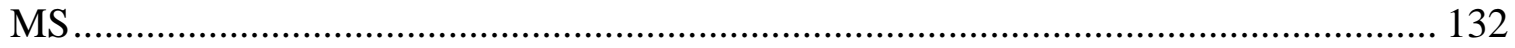

Figure 37. TATP Headspace Using SPME-GC-MS ............................................... 134

Figure 38. Equilibrium Time and Extraction Temperature Experiments for the

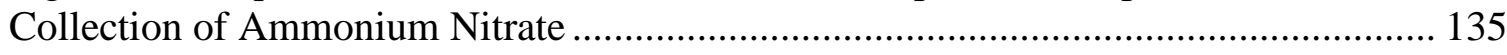

Figure 39. Flow Rate Optimization for AN at 30 seconds ..................................... 136

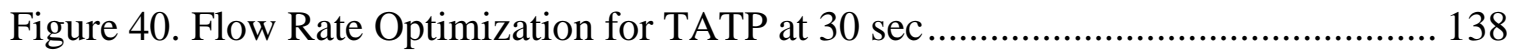


Figure 41. AN Derivative Product: Sampling Time optimization ............................... 139

Figure 42. TATP: Sampling Time Optimization .................................................... 140

Figure 43. AN Carbamate Product: Static Collection of VOC Over Time ................... 141

Figure 44. Static vs. Dynamic of Ammonium Nitrate Derivative ............................... 142

Figure 45. Comparison Between Different Dynamic and Atatic Collection Modes for Carbamate Derivative from AN ......................................................................... 143

Figure 46. Peresistence of Target Volatile Over Time Using Different Containment

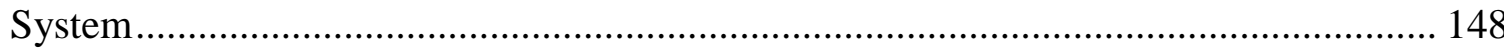

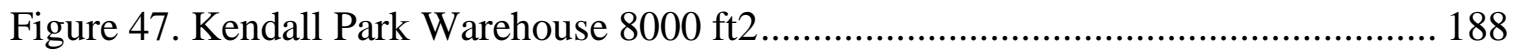

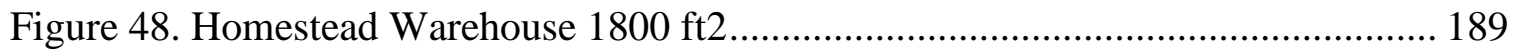

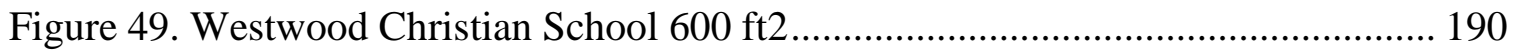

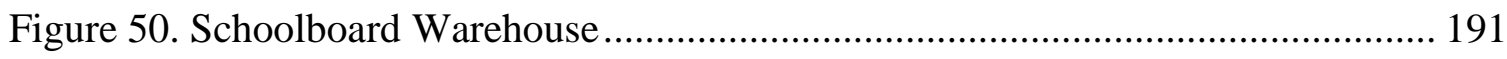

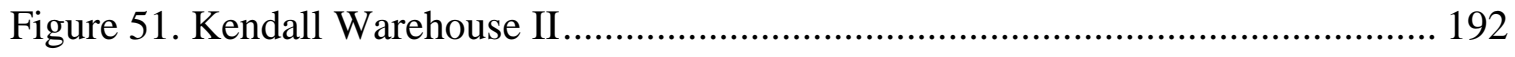




\section{ACRONYMS AND ABBREVIATION}

A

2,4-DNT

$2 \mathrm{E} 1 \mathrm{H}$

$\mathrm{ACN}$

ALS

AN

ANFO

ANOVA

ASA

ASTM

BZA

$\mathrm{CE}$

COMPS

CSA

DEA

DMNB

ECD

ESIS

FDA

FDSS

FN

FO
Analyte

2,4-dinitrotoluene

2-ethyl-1-hexanol

Acetonitrile

Automatic liquid sampler

Ammonium nitrate

Ammonium nitrate/Fuel oil

Analysis of variance

Acetyl salicylic acid

American society for testing materials

Benzoic acid

Capillary electrophoresis

Controlled odor permeation system

Control Substances Act

Drug Enforcement Administration

2,3-dimethyl-dinitrobutane

Electron capture detector

Externally sampled internal standard

Food and Drug Administration

Federal-wide Drug Seizure System

False negative

Fuel oil 


\begin{tabular}{|c|c|}
\hline FP & False positive \\
\hline GC & Gas chromatography \\
\hline GC-MS & Gas chromatography-mass spectrometry \\
\hline HHS & Health and Human Services \\
\hline HSCS & Human scent collection system \\
\hline HSD & Honestly significant difference \\
\hline HS-SPME & Headspace solid phase microextraction \\
\hline IED & Improvised explosives devises \\
\hline IMS & Ion-mobility spectrometry \\
\hline INCB & International Narcotics Control Board \\
\hline IOM & Institute of Medicine \\
\hline IR & Infrared spectroscopy \\
\hline IS & Internal standard \\
\hline LDPE & Low-density polyethylene \\
\hline LSD & Lysergic acid diethylamide \\
\hline $\mathrm{MeCl}_{2}$ & Methylene Chloride \\
\hline MS & Mass spectrometer \\
\hline MSD & Mass selective detector \\
\hline NG & Nitroglycerine \\
\hline NIST & National Institute of Standards and Technology \\
\hline NMR & Nuclear Magnetic Resonance \\
\hline NPV & Negative predictive value \\
\hline NSDUH & National Survey on Drug Use and Health \\
\hline
\end{tabular}




\begin{tabular}{|c|c|}
\hline ORT & Odor recognition test \\
\hline PC & Positive control \\
\hline PPV & Positive predictive value \\
\hline PTFE & Polytetrafluoroethylene \\
\hline RSD & Relative standard deviation \\
\hline RT & Retention time \\
\hline SA & Salicylic acid \\
\hline SPME & Solid phase microextraction \\
\hline STRIDE & $\begin{array}{l}\text { System to Retrieve Information from Drug } \\
\text { Evidence }\end{array}$ \\
\hline TATP & Triacetone triperoxide \\
\hline $\mathrm{THC}$ & $\Delta-9$ tetrahydrocannabinol \\
\hline TLC & Thin layer chromatography \\
\hline $\mathrm{TN}$ & True negative \\
\hline $\mathrm{TP}$ & True positive \\
\hline TPI & Temperature programmable inlet \\
\hline TPSA & Topological polar surface area \\
\hline UPS & United Postal Service \\
\hline USA & United States of America \\
\hline VOCs & Volatile organic compounds \\
\hline
\end{tabular}




\section{INTRODUCTION}

Drug trafficking is a global illicit trade involving the cultivation, manufacture, distribution and sale of substances which are subject to drug prohibition laws $(1,2)$. Although estimates of the extent of illicit drug production, distribution and consumption vary enormously, and are often contingent upon the methodology and political orientation of the observer (3), the continuous rise in drug trafficking over time has generated a growing concern for which detector canines have become an integral part of law enforcement. In fact, dogs contribute more to the execution of law enforcement objectives than sophisticated law enforcement technology (4). Specially trained detection canines (5) are commonly used by law enforcement to detect narcotics, ignitable liquids residues, explosives, currency, human remains, human scent, and by fire investigators to detect the presence of accelerants $(6,7)$. Over the past decade the United Nations Office on Drugs and Crime have presented in the World Drug Report the five most frequent drugs involved in trafficking offenses: marijuana, cocaine, heroin, amphetamines and new psychoactive substances. The present research discuss the development of training aids for two of the most frequently involved drugs in drug trafficking; marijuana and heroin, for canine detection. Training aids are meant to be used as a tool designated to improve the level of detection as they will allow daily trainings, will avoid any health hazard concerns, legal possession paper work, and challenges while been transported. These aids will improve the proficiency of detection which in turn will benefit the law enforcement community by providing a trustworthy tool that will help on the efforts to combat the illegal trafficking of illicit substances. A training aid is composed of non-illicit, non-hazardous chemical compound or a mixture of compounds that mimic the odor of a substance. On the basis of 
previously reported research, it is known that detector canines do not alert to the actual illicit substance but to a compound associated with it (8). Because canines alert response to compounds expelled by substances, the headspace of two controlled substances; heroin and marijuana was evaluated to determine the constituent or signature odor(s) responsible for the alert response of the canine (9). Prior to the evaluation of a substance one must guarantee the use of the most effective method of analysis to ensure an accurate response. Therefore, for the present study different parameters affecting the extraction of volatiles from the headspace of the illicit substances were evaluated. To isolate the compounds found in the headspace of the samples, solid phase microextraction (SPME) was used in conjunction with gas chromatography-mass spectrometry (GC-MS). Solid phase microextraction has been proved as an effective method for extracting volatiles from the headspace of explosives and other materials (9-12) thus it was used to complete this task. Selecting the appropriate SPME fiber coating is of the utmost importance to ensure an appropriate analyte recovery, as different fiber coatings varying in polarity result in different compounds being extracted. As an equilibrium extraction technique, SPME calibration depends on the establishment of an equilibrium between analyte concentrations in the extraction phase and the bulk of the sample (13). Therefore, after selecting the most suitable fiber for the extraction, both equilibrium time and extraction times were studied. Once the optimized parameters for the extraction of volatiles were selected, it was possible to sample a variety of real illicit substances provided by the Drug Enforcement Administration (14).

The characterization of volatile organic compounds (VOCs) from the illicit substances provides a specific information data of those common and/or abundant compounds 
potentially responsible for the alert response of the canines. Predominant compounds found in various types of marijuana and heroin samples were tested in the field using canines. Odor recognition testing (ORT) developed for the determination of the compound(s) were conducted single blind, where neither the canine teams nor the canine trainer knew where the potential mimics were hidden, but the evaluator did know. The study was conducted to eliminate bias from handlers who can skew the results. Upon identification of the active odor signature, optimization of the training aids was performed to ensure the delivery of the target odor to the canine in the most efficient manner.

Additionally, an alternative for the rapid development of training aids for the evolving improvised explosive devices (IEDs) has been evaluated. The frequent use of these devices have developed substantial concern worldwide requiring much more attention to legal chemicals such as ammonium nitrate, which could potentially be used in mixtures to create these devises. The collection system function by means of a vacuum mechanism that will suction volatiles from the material it is exposed to, the volatiles will be absorbed by a gauze pad which could later be presented to detector canines for training. The device has various settings that are meant to be modified depending on the explosive class being analyzed. In this section, the device's settings will be evaluated to determine how the flow rate and sampling time affect the amount of volatile(s) extracted, and to select the most suitable parameters depending on the explosive class.

The primary goal of the present research study aims to support the law enforcement community by developing a training aid for heroin and marijuana. The training aids will serve as a proficiency maintenance tool for the canine teams, as the developed aids are not controlled, avoiding any restrictions to train on daily basis. Additionally, aids are non- 
hazardous materials for safety training, avoiding any health hazards that may adversely affect human and/or canines' health. Once the training aids were optimized, the goal is to increase the number of detection canine teams that can reliably detect concealed drugs and explosives. 


\section{LITERATURE REVIEW}

Despite all efforts from the Drug Enforcement Administration and other law enforcement agencies to control and dismantle drug trafficking organizations, it is still a significant problem nationally (14). In February 2015, a man was sentenced to 10 years and one month in federal prison after trying to distribute approximately $100 \mathrm{~kg}$ of marijuana in 2014 (15). The man not only was a trafficker himself but had also conspired with a group of associates to ship thousands of pounds of marijuana via United Postal Service (16) from the Texas border to Sarasota, Hillsborough, and Polk Counties. Evidence presented revealed that in 2014 , he had arranged the shipment of at least 1,860 pounds of marijuana worth at least $\$ 927,000$ of related drug proceeds back to his suppliers in Texas (2). On the basis of the information provided by the Federal-wide Drug Seizure System (FDSS), marijuana along with heroin are two of the most frequently seized drugs within the jurisdiction of the United States. In July 17, 2015, DEA in collaboration with other government agencies intercepted what was meant to be an import transaction of kilograms

of heroin from Afghanistan to the United States worth thousands of dollars. As a result of the recent increase in supply and decline in cost, heroin is becoming one of the most popular drugs on the market.

\subsection{Drugs}

\subsubsection{History}

Drugs were introduced to the world for their medical and/or spiritual uses rather than recreational, but today their primary use has turned out to be the latter (17). Since the early 19th century when drugs became symbols of youthful rebellion, social upheaval, and 
political dissent, our society has confronted the problem of drug abuse and addiction. Consequently, law enforcement measurements were established. First anti-opium laws in the 1870s, followed by anti-cocaine laws, in the South in the early 1900s and antimarijuana laws, in the Midwest and the Southwest in the 1910s and 20s (18).

Even more strict measurements were taken when the United States enacted additional laws concerning consumer protections and public health. In the early 1900s the nation banned additive drugs with the help of the International Opium Convention who lead international agreements regulating trade (19). The Food and Drugs Act of 1906 was the beginning of over 200 laws concerning public health and consumer protections. However, it was difficult to oversee all the laws at once, thus the nation's problem was not been properly attended. In an attempt to have a more controlled system, President Richard Nixon signed the Controlled Substances Act (CSA) in 1970. This act essentially combines all the preexisting federal drug laws into one single statute. In other words, the purpose of this act is to regulate the manufacture, possession, importation, use and distribution of illegal substances (20).

\subsubsection{Drug Scheduling}

The legislation created five Schedules divided by different qualifications that the substance must meet to be included in each. Although the qualification of the drugs depends on both the potential for abuse and the acceptable medical use in treatment the drug has, what determines the scheduling of the drug is the rate of abuse. As the schedules increase in number, the drug potential of abuse decreases and so are the penalties involved if a subject is detained for illegal possession. Drug Enforcement Administration and the 
Department of Health and Human Services (HHS) are currently the two agencies allowed to determine which substances are included, removed or changed from the various schedules (2). The specific classification of any given drug or other substance is usually a source of controversy, as is the purpose and effectiveness of the entire regulatory scheme.

\subsubsection{Schedule I}

Substances that are part of this category are those that have high potential for abuse. As this drugs have no current medical use in treatment in the United States, no prescriptions are allowed to be written. Schedule 1 drugs are considered to be highly dangerous when used without medical supervision in comparison to those from other schedules. Drugs in this schedule include e heroin, gamma hydroxybutyric acid (GHB), lysergic acid diethylamide (LSD), marijuana, and methaqualone (2).

\subsubsection{Schedule II}

Schedule II drugs are those considered to have high potential for abuse but to a lower degree when compared to Schedule I drugs. Although there is still likely possibilities for its abuse, there are some accepted medical uses in the United States. The abuse of Schedule II drugs leads to physical and/or psychological dependence and it is considered dangerous. Drugs on this schedule include: morphine, phencyclidine (PCP), cocaine, methadone, hydrocodone, fentanyl, and methamphetamine (2).

\subsubsection{Schedule III}

Substances included in this schedule are those considered to have less potential for abuse and thus have accepted medical uses. Additionally, when abused, its misused will lead to moderate to low physical and/or psychological dependence. Drugs in this schedule 
include: products containing less than 90 milligrams of codeine per dosage unit (Tylenol with codeine), Anabolic steroids, codeine, and some barbiturates (2).

\subsubsection{Schedule IV}

Schedule IV drugs are considered those with low potential for abuse relative to the drugs in Schedule III this in turns, have low risk of dependence. Drugs on this schedule include: Xanax, Soma, Darvon, Darvocet, Valium, Ativan, Talwin, Ambien, Tramadol (21).

\subsubsection{Schedule $V$}

Drugs, substances, or chemicals included on this schedule are those with the lowest potential for abuse in comparison with previous schedules. These drugs have a currently accepted medical use in treatment in the United States and consist of preparations containing limited quantities of certain narcotics. Schedule V drugs are generally used for antidiarrheal, antitussive, and analgesic purposes (e.g. cough preparations with less than 200 milligrams of codeine or per 100 milliliters (Robitussin AC), Lomotil, Motofen, Lyrica, Parepectolin) (21).

\subsubsection{Classification}

Generally, a controlled substance is a drug or chemical that when consumed has a detrimental effect on a person's health and well-being. Therefore, all controlled substances' manufacture or possession is regulated by the government through CSA. The Control Substances Act classifies drugs into five categories: narcotics, stimulants, depressants, hallucinogens and anabolic steroids. Narcotics are known for their capabilities to induce dulled senses and alleviate pain but when excessive doses are supplied it can cause stupor, 
coma, or convulsions (22). Today, narcotics are associated with opiates, opioids and their synthetic substitutes, depending on their medical usefulness, abuse potential, safety, and drug dependence profile, opioids can vary in schedules from I-IV (2). In contrast, stimulants, as the name suggests, speed up the body's system. If high-doses are used symptoms such as agitation, hostility, panic, aggression, and suicidal or homicidal tendencies are encountered (2). As many stimulants are known to have legitimate medical uses, these drugs are often scheduled between Schedule II-IV. Depressants are the complete opposite, in fact, these are often referred to as "downers". These drugs are synthetic products that when consumed will put you to sleep, relieve anxiety and muscle spasms. The use of depressants can be fatal, if these drugs are used in high doses or mixed with alcohol or other drugs the heart rate and breathing can decrease enough to cause death (2). Most depressants are controlled substances that range from Schedule I-IV. Hallucinogens can be naturally produced from plants and fungi or synthetically produced in illicit laboratories. This group of drugs is known for their ability to alter human perception and mood. Deaths related to Lysergic acid diethylamide (LSD) overdose is extremely rare however, the side effect after their consumption can result in suicide, accidents, and dangerous behavior, or as a result of the person inadvertently eating poisonous plant material (2). Lastly are the anabolic steroids, which are synthetically produced alternates of the male hormone testosterone. These substances are well-known by athletes or body builders as they are commonly used among them to enhance muscle growth, increase athletic performance, or improve physical appearance (2). However, a small amount of steroids are approved by physicians to treat some patients therefore this category is associated with Schedule III. As it has been described, each class has 
distinguishing properties, but drugs within each class often produce similar effects. Out of all the categories previously mentioned, marijuana (hallucinogen) and heroin (narcotic) are two substances that tend to define the drug problem over time as these are two of the most trafficked and abused drugs (23).

\subsubsection{Marijuana}

Marijuana is the general term used for a natural harvested product derived from the plant Cannabis sativa $L$, and specifically for the leaves and flowering tops of those plants (18). The origin of cannabis is not entirely clear as it has been grown for thousands of years. The reason for its abundance around the world is due to the fact that cannabis seeds can be carried by the wind and in bird droppings (24). What has also complimented the great displacement of the seeds, is cannabis' growing conditions, besides requiring enough sunlight and food for it to flourish, its only necessity is stability in temperature. Cannabis can be grown almost anywhere as long as the temperature is not constantly cold. Human intervention on marijuana growth have also made possible its spread worldwide because cultivators have managed to artificially mimic the perfect conditions to grow the plants in areas where the plant would not have previously existed. Today, it is estimated that there are twelve or possibly more different cannabis strains growing wild in countries that have not culled the plant (24).

Although chemical research on marijuana have taken place for about a 150 years now, it was not until 1970s that the active component responsible for most of its psychoactive activity; delta-9 tetrahydrocannabinol (THC) (Figure 1) was determined (25). 


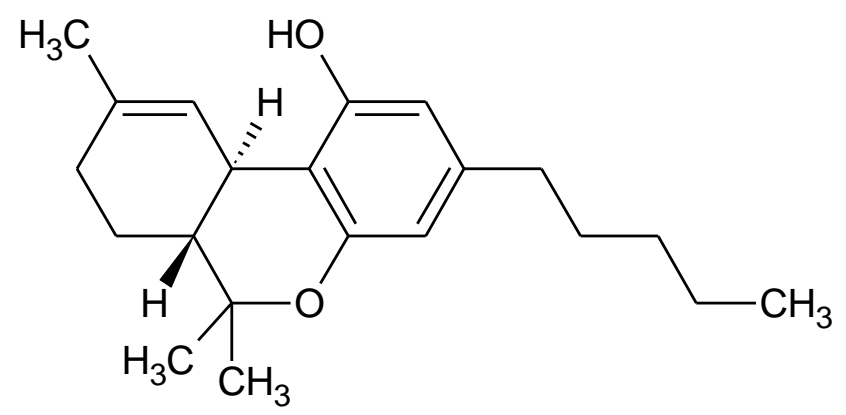

Figure 1. Tetrahydrocannabinol (THC)

Tetrahydrocannabinol is a resin insoluble in water most effective delivery method is inhalation, through this means users can regulate the drug dosage by adjusting the frequency and depth of inhalation (26). Tetrahydrocannabinol can also be consumed orally but absorption takes a longer time since it has to breakdown before getting into the bloodstream. Cannabinoids in marijuana act as an antagonists and attaches to a specific sites in the brain called cannabinoid receptors ( $\mathrm{CB} 1$ and $\mathrm{CB} 2$ ), generating a series of cellular reactions that eventually activates the "high" effect when users consume the drug (27). THC has most binding affinity to CB1 which is found in many areas of the brain, central nervous system, connective tissues, gonads, glands, and related organs whereas $\mathrm{CB} 2$ receptors are mainly expressed on $\mathrm{T}$ cells of the immune system, on macrophages and B cells, and in hematopoietic cells. This is the reason why consumption of cannabis results in significant relief from pain, nausea, or depression while delivering a strong euphoria to lifestyle users (28). 


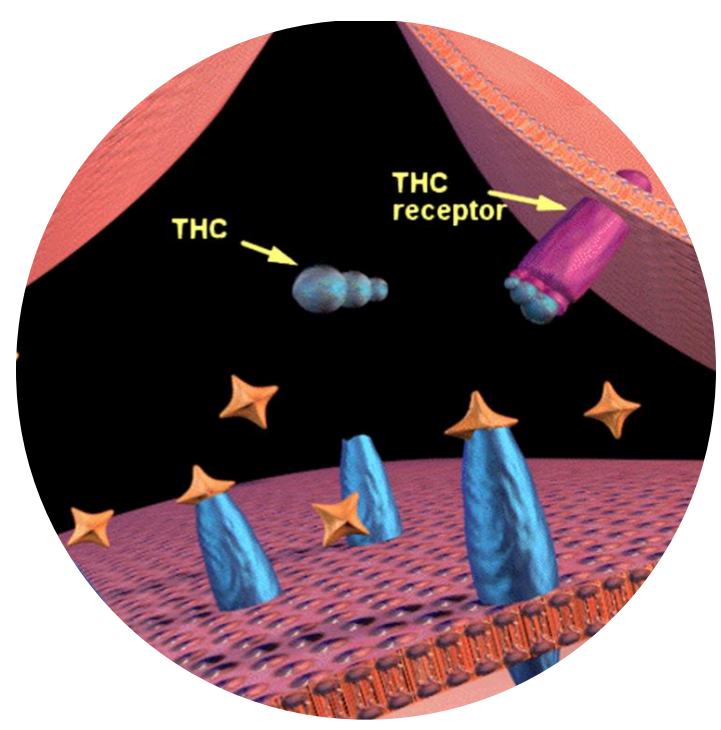

Figure 2. THC binding to cannabinoid receptor (CB1) (30)

Marijuana remains classified as Schedule 1 drug under CSA, even though today, 23 states, the District of Columbia and Guam allow public medical use of marijuana after the Obama Administration sent a memo in October 2009 discontinuing prosecution to those who distribute marijuana for medical purposes in accordance to state law (29). "Scientific data indicate the potential therapeutic value of cannabinoid drugs, primarily $\mathrm{THC}$, for pain relief, control of nausea and vomiting, and appetite stimulation; smoked marijuana, however, is a crude THC delivery system that also delivers harmful substances (30)."

Outdoor marijuana cultivation sites are becoming increasingly common. The Department of Agriculture Forest Service and the Department of the Interior reported the eradication of over 4 million marijuana plants from U.S. lands in 2008. In recent decades, marijuana growers have been genetically modifying their plants to have greater amounts of THC, the main ingredient found in the Cannabis plant. The average potency of tested 
marijuana from federal seizures have doubled from 1983 to 2009 (31). These have become a threat to the environment, public health, national security, transportation and educational success. In fact, the National Survey on Drug Use and Health (NSDUH) indicates that 78\% of the 2.4 million people who began using marijuana in the last year were aged 12 to 20 when the brain has not fully been developed and will therefore affect both educational and life outcomes (32). For these and many other reasons, the current administration together with federal partners, are working hard to reduce the use and trafficking of marijuana, through the development of strategies that are meant to integrate the principles of prevention, treatment, and recovery (33).

\subsubsection{Heroin}

Heroin, a Schedule I drug, is a narcotic synthesized by the acetylation of morphine, a naturally occurring substance that is extracted from a flowering plant called opium poppy. Opium is the product obtained from the poppy plant Papaver somniferum, a plant mainly grown in mountainous areas. After the flower of the poppy plant opens, a flowing juice released from the bulb is let to dry by the wind and subsequently boiled until it becomes raw opium called "black" (23). Black is later refined to a morphine base that can be later transformed to heroin by means of a chemical process (34) described below (Figure 3). Chemically know as diacetylmorphine, heroin was discovered in 1898, initially thought to be the cure for addiction of other opiates (35). 


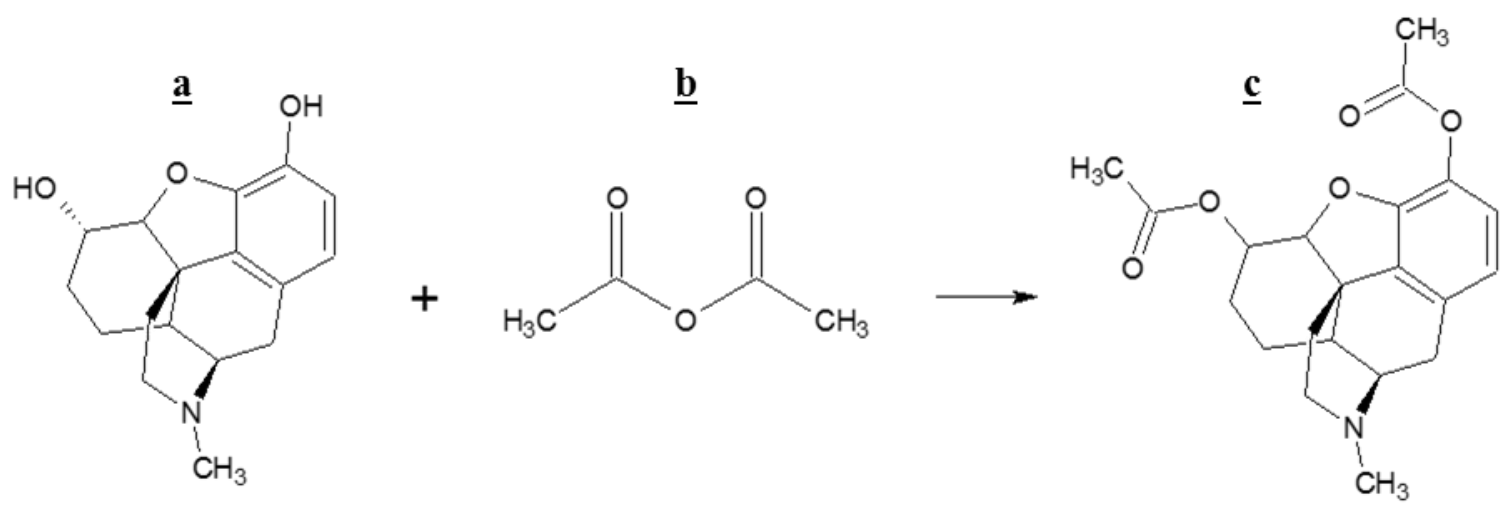

Figure 3. Chemical Synthesis of Heroin (a) Morphine (b) Acetic anhydride (c)
Diacetylmorphine (36)

Heroin appears as a white or brown powder depending on the impurities it may have after it is manufactured or because of the presence of additives. Although this product can be smoked, sniffed or injected, the latter delivery method predominates in efficiency as it has a long-lasting effect in comparison with the rest (23). However, the fear of HIV infection, hepatitis C, septicemia (blood poisoning), endocarditis (inflammation of the heart lining), skin abscesses, or tetanus after sharing needles, have made snorting and smoking a most common way to ingest the substance (37).

The agent responsible for the psychoactive effects in heroin is the parent compound, morphine. Once the heroin is consumed, it is transported to the brain through the bloodstream. In the brain, enzymes will convert heroin to morphine where they also get attached to opioids receptors causing a decrease in body pain, pleasure and/or euphoria (36). These receptors are located in areas of the brain such as part of the cerebral cortex, the VTA, nucleus accumbens, thalamus, brainstem, and even lower in spinal cord (38). The consumption of prescribed and illegal opiates such as heroin, cause euphoric effect, 
analgesia, and sedation after the morphine is produced in the body following heroin metabolism.

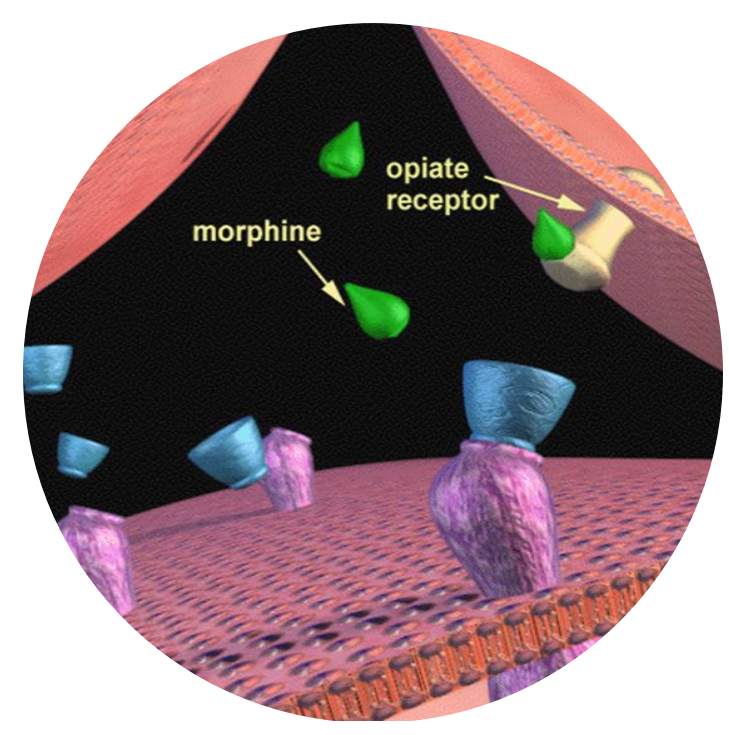

Figure 4. Opiates binding to opiate receptors (30)

Heroin consumption has become more popular over the years. According to the NSDUH, in 2012 about 669,000 Americans reported using heroin, a number that has been on the rise since 2007 (39). Law enforcement reports indicate that the reason for this increment is due to the vast availability in response to higher demand, evidenced by high wholesale purity, low prices, increase levels of abuse and higher number of reported overdose deaths (40). For instance, prescription opioids are typically more expensive than heroin therefore, abusers are switching to heroin as the same effects can be obtained with half the amount and price. The fact that criminal organizations are actively producing and transporting this drug threatens the United States as heroin availability keeps on rise and it is likely to continue increasing in the near term. 


\subsubsection{Trafficking}

Today, marijuana, heroin, alcohol, tobacco, and other drugs continue to have a worldwide medical and social impact. Many researchers deal with getting a more advance explanation(s) for the persistence of illicit drugs, while several advocate a more intrusive, harsher (41). Despite decades of intensified antinarcotics efforts, more drugs are being produced and distributed as a response of high demand due to increase in potency and affordability. In fact, drug illegal market represents one of the world's largest and lucrative business (42). Marketing and trafficking of drugs have become a global phenomenon (43) as regardless of where illicit substances are produced, smugglers find the way to get them into the country (44).

Special attention must be given to the vast number of transportation methods and routes taken to complete a smuggling transaction. For instance, in 2009 it was reported that from January through November, U.S. seizures of illegal drugs in transit exceeded 1,626 tons, which clearly indicates ability of smugglers for transportation (45). Trafficking of illicit drugs is typically conducted using commercials trucks and/or private or rental cars through immense areas of desert and mountainous landfills making challenging the capture of the people conducting the offense. Because of the active work of law enforcement to control this trades, smugglers have opted to change routes declining the amount of seized drugs. Therefore, prompt action needs to be taken to avoid the decrease of seizures but rather cause an increment of it.

Marijuana dominates the drug market in terms of cultivation, production and consumption. Although cannabis is grown all over the world, 55\% of its production correspond to the Americas. Cannabis, including the herb, resin, and oil, ranks first among 
all illicit substances that are seized globally (23). Illicit traffic of cannabis flows mainly from Mexico to the United States and, to a lesser extent from Canada to the United States (23). Heroin, on the other hand, was mainly produced in Afghanistan for as long as to 2008 accounting for more than $90 \%$ of the global production. A prime example of heroin flexibility in production characterized by how its manufacture can be move from one region to another to please the necessity of its demand. Heroin trafficking in the United States have move a long way, for instance, back in 1972 as response of its prohibition in Turkey, Mexican heroin was brought to fill the gap (23).

\subsection{Explosives}

Recently, bomb threats targeting the school system, airports, and other public locations have become the highlight on the news $(46,47)$. In 1995, the United States was shaken when a domestic act of terrorism occurred in Oklahoma City at the Alfred P. Murrah Federal Building; 168 people were killed and many injured, it was later revealed that the bomb consisted primarily of explosive-grade ammonium nitrate fertilizer (48). Moreover, as recent as March of this year, Brussels was also victim of an unfortunate attack by Islamic State where many were killed and injured after attackers detonated what was revealed to be $44 \mathrm{lbs}$ of ammonium nitrate bomb (49). Additionally, later while performing a search in the house linked to the prosecutors, "more than 30 pounds of another high explosive known as TATP was found along with 150 liters of acetone and 30 liters of hydrogen peroxide, both chemicals that can be used to make bombs" (50). This commotion promotes the improvement of new approaches for a faster development of explosives training aids for canine detection. New explosive recipes are frequently released and the analysis to identify 
the active odor (5) of these materials for the development of canine training aids is considered to be time consuming. Collection systems have been developed for the collection of volatiles from different materials through vacuum technology that is further collected into gauze pads. This system has been evaluated as a potential remedy to quickly develop non hazardous, portable, and safe training aids for canine detection teams.

\subsubsection{History and classification}

Explosives were first introduced by mistake when an alchemist produced black powder after confusing a chemical reaction intended to separate gold from silver (51). Nowadays, creating new explosives is completely different, as it has become common to see new recipes more often. The American Society for Testing Materials (ASTM) defines energetic materials (e.g. explosives, propellants, pyrotechnics) as a compound or a mixture of compounds containing fuel and oxidizer that when react release large amount of energy and gas that leads to combustion, explosion and/or detonation (52). Explosives can be classified into six different classes depending on their chemical properties, performance and uses. When divided onto active functional groups classifications are: acid salts, aliphatic nitro, aromatic nitro, nitrate ester, nitramine and peroxide (53). Although explosives can be classified based upon the molecular group they contain, this classification does not give any information regarding the performance of the explosive. A better classification is according to their rate of decomposition and the susceptibility to initiate (54) (Figure 5). Both characteristics depend on how stable the material is when exposed to extreme environmental conditions. For instance, low explosives will burn rapidly while high explosives will undergo detonations. High explosives are further divided 
into primary and secondary explosives. Primary explosives are those unstable materials that will detonate easily when exposed to nonexplosive means such as fire, sparks, heat, pressure, shock, and percussion. The reason for this explosive class to be called primary is due to its ability to denote without the need of an outside energy opposed to secondary explosives a class that does requires assistance to detonate. The fact that primary explosives easily detonate and produce high amount of heat and/or shock, make them a source used to initiate a secondary explosive. Although secondary explosives are less sensitive than primary explosives, these are more powerful than the latter. In fact, some secondary explosives are so stable than even a rifle bullet can go through it and it will only set them on fire without detonating (55). Secondary explosives are also mixed with tertiary explosives to explode, as tertiary explosives are insensitive to shock and can not reliable detonate otherwise.

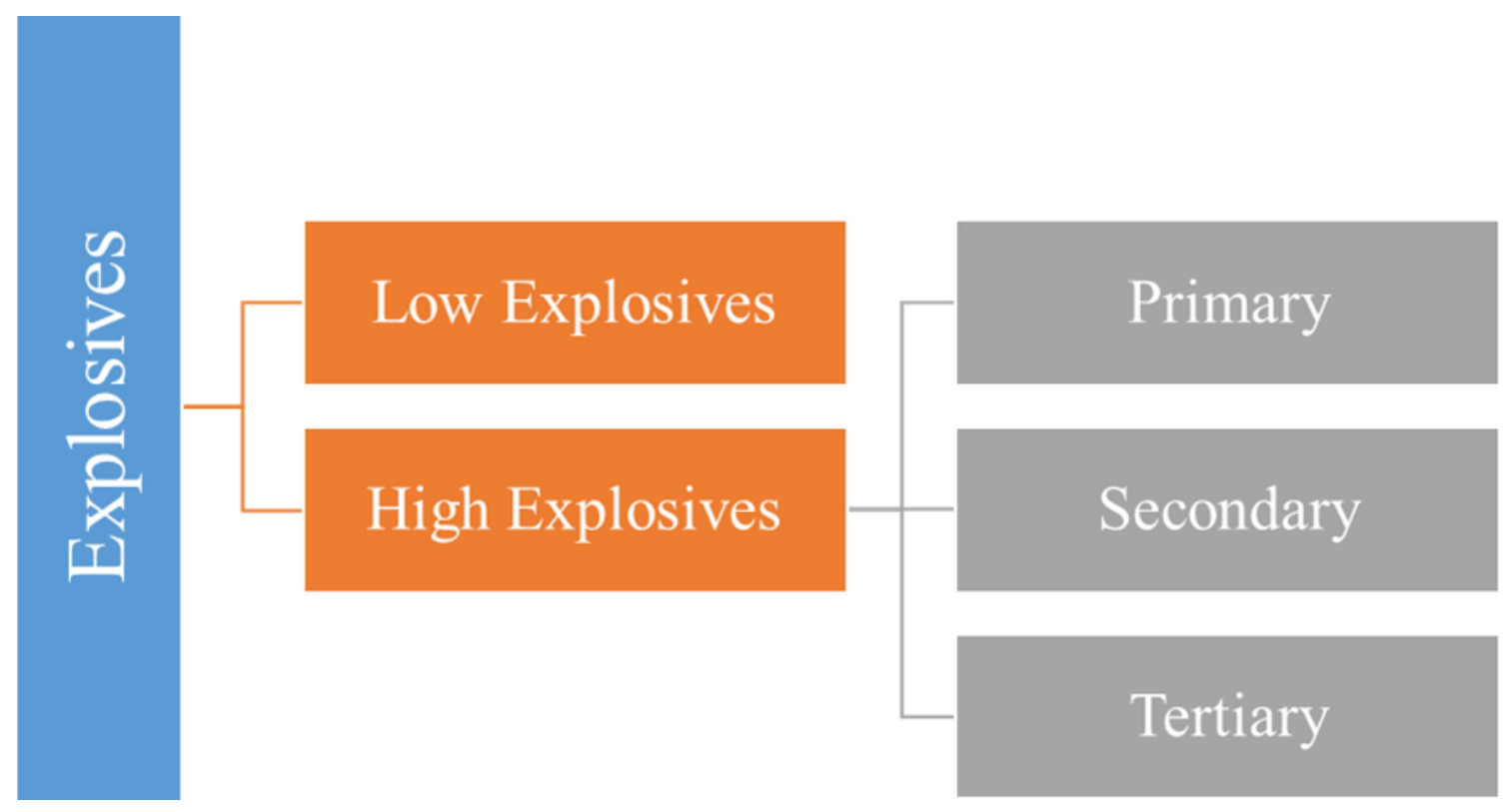

Figure 5. Classification of Explosives 


\subsubsection{Triacetone Triperoxide (TATP) (56)}

Triacetone triperoxide is a primary explosive first synthesized in 1895 by Richard Wolffenstaein (57). This compound was accidentally discovered while Wolffenstaein was working on a project involving hydrogen peroxide treatment on coniine alkaloid. After letting stand a mixture of hydrogen peroxide and acetone for several days, a white crystal precipitate, now recognized as TATP, was discovered. His optimized method, i.e., reaction of acetone with $\mathrm{H}_{2} \mathrm{O}_{2}$ in acidic medium (used to increase TATP yield), is still a common method for obtaining TATP (57). As depicted in Figure 6 TATP structure is based on a cyclic peroxide bridged structure in which out of total of nine ring atoms, six are oxygen atoms (58).

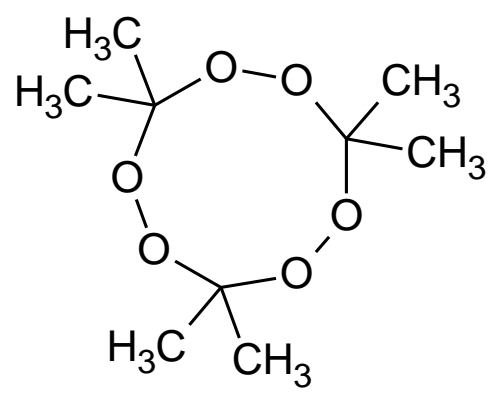

\section{Figure 6. Triacetone Triperoxide (56) Molecule}

Triacetone triperoxide is a very volatile nonhydroscopic colorless crystals which appears white when it is grounded (59). In the first half of the twenty century, TATP found use in several applications including as a substituent of highly toxic mercury fulminate. However, due to its low thermal stability, and its sensitivity to open flames and stress, TATP has not been extensively used industrially or for military applications (60). Conversely, in the past, TATP has been used by terrorists in many bombs attacks from which the most outstanding attempt was by Richard Reid who put the material in his 
sneaker in an attempt to ignite a trans-Atlantic jetliner in 2001 (61). But more recently, back in November 2015, 129 people were killed while 99 were seriously injured after eight attackers set off packed bomb vests containing TATP. However, since TATP does not contain nitrogen it makes it difficult to detect by security scanners (62).

\subsubsection{Ammonium Nitrate (AN)}

Ammonium nitrate (AN) was discovered in 1659 by J.R. Glauber and has become one of the most common and commercially important compounds used to create explosive materials (63). By 1917, 92\% of the explosives produced by British coal productions were based on ammonium nitrate (64). The reduce the cost of the explosives, the industry started adding cheaper compound of AN but it played against them as it reduced impermeability of the product. It was not until the industry improved the packaging of the material, that the product stability increased due to increment on its water resistance. The most notable composition was Ammonium Nitrate Fuel Oil (ANFO), where AN serves as the oxidizing agent for the fuel. This emulsion is waterproof, safer than dynamite, simple and cheap to manufacture (64). Ammonium nitrate is so called common mainly because is the key component in numerous fertilizer, cleaning solutions and industrial explosives. The role of AN in fertilizer is mainly to provide $\mathrm{N}_{2}$ in form of $\mathrm{NH}_{3}$ and nitrate ion while as in explosives it works as the source of oxygen (oxidizer) that will combined with the fuel and release energy (63). As seen in Figure $7 \mathrm{AN}$ is manufactured by neutralization of nitric acid with ammonia. 

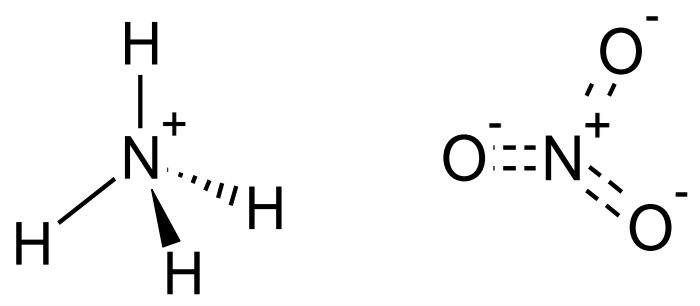

Figure 7. Ammonium Nitrate (AN)

Ammonium Nitrate appears as a solid, dense flakes or grains. It is the least sensitive of military explosives (65) in fact, as a blasting agent ANFO requires a primer to be initiated. As a result of the low cost and cratering effects nowdays, ANFO is the explosive of choice for demolition operations. Although pure AN is stable at room temperature, the explosive character of AN by itself was tragically found through disasters involving AN alone in explosions. The most well known incidents include Upper Silesia (1921), Oppau, Germany (1921), Brest, France (1947), Texas City, USA (1947), Toulose, France (61).

\subsection{Detection of Illicit Substances}

In forensics, different analytical techniques that are known to have a high degree of certainty are used to detect illicit substances in the laboratory and in the field. Major indicators of illicit substances include VOCs and biological markers, the analytical approach undertaken will depend on the purpose of the analysis. The analyst must decide not only how to test but also how many samples to test. In general, when a sampling its being conducted, it is important for the method development to go accordingly with the purpose of the study as it affects the reliability of testing. A well-designed analysis protocol is likely to involve physical separation between the analyst and the sample, the careful 
handling to avoid contaminants, the inclusion of appropriate blank and positive control, and environmental monitoring as it might affect the sampling technique being used.

\subsubsection{Sampling Techniques}

The first technique to be considered when designing a method is the sampling technique. Forensic laboratories use preliminary tests to identify the evidence followed by analytical methods used to provide definitive confirmation. Rapid testing methods usually consist of colorimetric testing. Such color tests are used to indicate the presence of an illicit substance in a bulk material by changing color. These presumptive tests are meant to be used by any personnel in the law enforcement as they have straightforward procedures that can be accomplished by simply following the manufacturer instructions. Examples of this type of test include, marquis reagent, which is one of the most popular color metric reagent used for the identification of opiates. The test is performed by scraping off a small amount of the substance and adding a drop of the reagent (which is initially near colorless). The results are analyzed by perception of the color of the resulting mixture, for example, if heroin is the unknown sample tested, the liquid will turn purple. Another example is Duquenois-levine reagent which is used for the identification of THC in cannabis products including resin and oil (66) it goes from violet to purple color in chloroform layer. Although these methods are quite assertive for each drug, a color test is simply used as a qualitative approach as they do not provide with any information regarding the concentration and purity of the illicit substance. Despite the fact that other methods might be more laborious, the tendency for more accurate and complete results is the extraction of target compounds followed by analytical techniques. 


\subsubsection{Analytical Techniques}

An analytical procedure involves several steps (1) separation, (2) quantification, and (3) data analysis, each of which must be executed correctly in order to obtain accurate and reproducible results (67). Prior to the separation of the sample, a sample preparation is often required to isolate the analyte, especially when working with trace levels of target analytes present in complex matrices. Nowadays, traditional sample preparation methods are often been avoided by scientists due to a number of drawbacks being substantially decreased or even eliminated by best practices and most recent developments. Problems associated to these traditional methods include but are not limited to the use of solvents, particularly a threat to the environment, and multistep procedures that often result in the loss of analyte during the process, which turned into a major source of error, affecting the quality of the analysis.

New developments have recently been applied to analyze forensic samples. An ideal sample preparation technique should be solvent-free, simple, inexpensive, selective, and compatible with a wide range of separation methods and applications (67). Headspace SPME has been demonstrated to be advantageous in identifying trace volatile components from forensic specimens without having to resort to long extraction times, heating of samples or using dynamic flow (headspace stripping) which can dramatically alter ratios of odor signature chemicals recovered (5). In this study, solid phase microextraction (SPME) was applied as the extraction technique to isolate volatile organic compounds (9) from illicit substances because not only it offers all of the characteristics previously mentioned, but also it is a portable technique perfect for field sampling as it is essential to complete this study. 


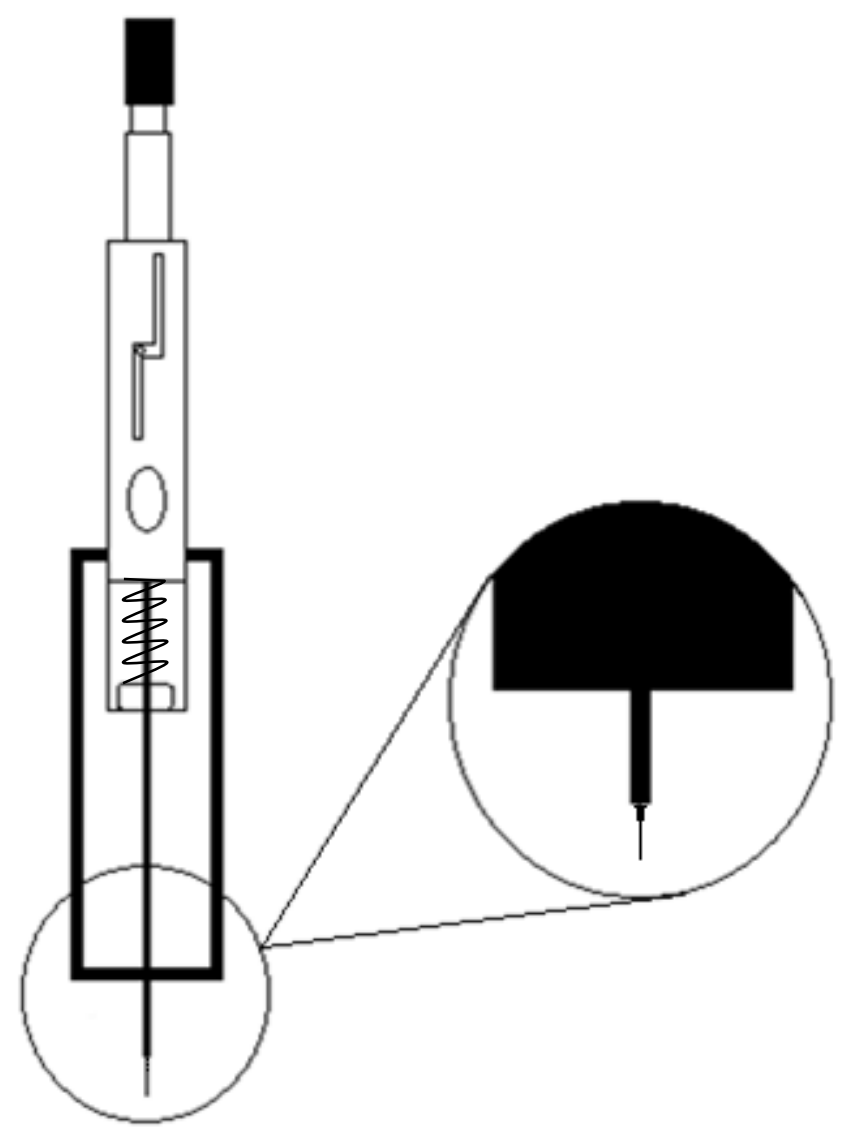

Figure 8. SPME Fiber and Holder Assembly

Solid-phase microextraction involves the use of a modified syringe that includes a polymer coating on a fused silica fiber. The fiber is attached to a metal rod, and it is protected with the metal sheath that covers the fiber when it is not in use. When the fiber is ready to be used, it can be pushed using the plunger and lock in place by sliding the plunger retaining screw through the Z-slot, this mechanism will exposed the fiber so that the extraction is performed. The extraction process involved in SPME consists of a twostage process, the first one being the partition of analytes between the sample and the fiber coating, followed by the desorption of the analytes from the fiber to the analytical 
instrument for separation and quantification (68). The most important step of this process is the adsorption or absorption of the analytes into the fiber. Thus, the selection of the fiber coating is essential to perform a suitable extraction. The affinity of the compounds to the coating will greatly depend on the analyte(s) contained in the sample therefore, a proper study and selection of the coating must always be performed.

Solid phase microextraction is a multistep equilibrium technique. The analyte recovery from samples can be done by any of two approaches (e.g. direct or headspace), depending on the type of compound(s) to be analyzed. The fiber can be either expose directly to the liquid sample or simply above the sample. Although SPME provides different techniques that could be performed, when studying VOCs, the extraction is limited to be studied through headspace method. For volatile compounds, once they are dissociated from their matrix they are easily the release into the headspace where they are extracted for analysis (67).

The theory behind how headspace solid phase microextraction (HS-SPME) works consists upon an equilibrium based on a three-phase system that includes the sample, the headspace above the sample, and the fiber coating. During extraction, analyte migrate between all three phases until equilibrium is reached. The equations that summarize this process are:

$$
\begin{gathered}
K_{h s}=\frac{C_{h}}{C_{s}} \quad \begin{array}{l}
\mathrm{K}_{\mathrm{hs}} \text { is defined as the distribution constant between the } \\
\text { concentration of the analyte in the headspace phase } \\
\left(\mathrm{C}_{\mathrm{h}}\right) \text { and the sample matrix }\left(\mathrm{C}_{\mathrm{s}}\right)
\end{array} \\
K_{f s}=\frac{C_{f}}{C_{s}} \quad \begin{array}{l}
\mathrm{K}_{\mathrm{fs}} \text { is defined as the distribution constant between the } \\
\text { concentration of the analyte in the fiber coating }\left(\mathrm{C}_{\mathrm{f}}\right) \\
\text { and the sample matrix }\left(\mathrm{C}_{\mathrm{s}}\right)
\end{array}
\end{gathered}
$$


The mass of the analyte (n) absorbed/adsorbed is then expressed as:

$$
n=\frac{K_{f s} V_{f} C_{0} V_{s}}{K_{f s} V_{f}+K_{h s} V_{h}+V_{s}}
$$

Equation 1

Where $\mathrm{C}_{0}$ is the initial concentration of the analyte in the matrix, and $\mathrm{Vf}, \mathrm{V}_{\mathrm{h}}$, and $\mathrm{V}_{\mathrm{s}}$ are the volume of the fiber coating, the headspace and the matrix, respectively. The three terms in the denominator express the capacity of the analyte in each phase. In general, this equation states that as long as the volume of fiber coating, headspace or sample are kept constant, the analyte extracted will be independent of the location of the fiber in the sample (12). Therefore, there is no difference between how the sample is extracted, directly or headspace. The selection of the extraction technique utilized will then depend on the type of sample been analyzed. Since is the purpose of this study is to evaluate the volatiles present in illicit substances, headspace sample is the sampling method chosen.

A variety of fiber chemistries are commercially available, and they need to be considered especially when sampling unknowns. The fibers vary in the fiber coating, film thickness, polarity, and absorption/adsorption mechanism. The manufacturer provides recommendations for general applications, but further analysis of the fiber chemistry must be considered as the chemical compounds extracted will greatly depend on the affinity they have with the selected fiber coating. Molecules can be associated to the coatings by means of polarity, as polar analytes will be attracted to polar phases. Therefore, polarity can help enhance the affinity of the coating for polar analytes by selecting a polar coating rather than a non-polar. Thus, the greater the polarity of the polymer coating, the more polar 
compounds can be analyzed at low concentrations. However, even though all fibers are bipolar at some degree, is the overall coating of the fiber what determines the polarity. Now, when a fiber is classified as bipolar it is mainly due to the extraction mechanism, adsorption. Adsorption type fibers generally contain pores or high surface areas where the analytes interact. These pores allow the extraction and retention of small and midsized analytes usually until either energy is applied or they are displaced by a solvent (69). In contrast, absorbent fibers' ability to retain an analyte, will merely depend on the fiber capacity determined by its thickness, as there is no competition involved but instead how fast the analytes migrate in and out the coating. Therefore, to improve the sensitivity of the extraction technique one must consider to increase the fiber thickness or length when working with large volume samples thus increasing the fiber capacity.

\subsubsection{Narcotics}

Forensic drug analysis rely on different test ranging from presumptive tests like spot tests, immunoassay, previously discussed, to detection techniques such as infrared spectroscopy (IR), or separation techniques like, high-performance liquid chromatography (HPLC), capillary electrophoresis (CE), thin-layer chromatography (TLC), GC, and a combination of these (70). The analytical tools and methods outlined here are quite dissimilar in design, type of sample addressed, and results produced. For instance, IR induces vibration excitation to create a unique fingerprint of covalently bonded atoms but limitations arises as the molecular weight of the compound will not be known nor the location of the functional group in the chemical compound. Additionally, IR is not recommended for the analysis complex mixtures as IR is best when a pure substance is 
analyzed as all the bands will assigned to a single molecule (71). HPLC have been widely used in the field of drug analysis however, since it is best coupled to UV, this technique is limited to those compound that have a UV absorbing chromophore. HPLC is a separation technique where samples is dissolved in a liquid mobile phase and is separated by means of the interactions with the stationary phase contained in the column. If a solid samples is subject to be analyzed, it must be dissolved and filtered to be injected, while depending on the case, liquid samples can be directly injected. Capillary electrophoresis (CE) is a special case of using an electrical field to separate the components of a mixture through the "opposite attract" principle, positive charged molecules will be attracted to the anode, while negative charged molecules will be attracted by the cathode (72). Nevertheless, this technique is limited by the amount of sample injected as it can be easily saturated. Thin layer chromatography is another separation technique used consisting on a stationary and mobile phase. The solid phase is a thin solid support that usually consists of Alumina or Silica while the mobile phase is a solvent that moves through capillary action right through the solid phase (73). Limitations associated with this technique is that is only a qualitative analysis that does not reflect the difference between enantiomers and some isomers. Although they all are able to uncover forensically relevant information, depending on the goal of analysis, it may or may not be suitable to fit the purpose. Therefore, special attention must be considered to the species been evaluated and/or the physical and chemical characteristic to select the best analytical that will allow a better discrimination and characterization of material evidence. 


\subsubsection{Explosives}

The existing technologies for explosives detection can be separated into two categories, bulk detection and trace detection (74). Methods for detecting trace explosives include chemical techniques such as ion mobility spectrometry (75), GC-ECD/MS, or optical techniques like surface acoustic wave. Ion mobility spectrometry for example, is a separation technique typically implemented as a stand-alone detector that is used at the airport to detect trace amounts of explosives. This separation technique will separate ionized molecules in the gas phase through a drift tube containing an electric field and is connected to a detector (76). However, this technique's selectivity is moderate in comparison to others and it may contain radioactive source (77). Surface acoustic wave on the other hand, is less specific and in the presence of other chemicals, this technique stands unreliable (77). On the other hand, bulk detector techniques ranges from X-Rays, neutrons, electromagnetic imaging, such as IR or microwave and electro-magnetic such as NMR. These methods identify chemicals based on characteristic absorption of particular wavelengths of light. Depending on the purpose of analysis is the technique to be considered for evaluation. All of these techniques have been well described in the literature. Therefore, this dissertation will only describes in more detail the technique that will be applied for the evaluation of VOCs from the headspace of several samples.

Nowadays, simplicity and speed of chemical analysis are attractive characteristics for all analytical procedures. Over the past few years, SPME have shown to be an excellent alternative for sample introduction in GC. Not only this combination reduces the timeresponse delivery but also the costs of operation in numerous domains (78). Perhaps its high efficiency and easy coupling with selective and sensitive detectors, gas 
chromatography remains a frequently selected separation technique (78). The key component for the development of training aids for canine detection has been the identification of the VOCs (signature odor(s)) released from the parent substances. It is known canines alert to a chemical(s) component in the headspace of the samples and not the parent drug itself however, the question is still which component in the headspace the canines alert to. Although several analytical techniques have previously been used, gas chromatography (GC) combined with mass spectroscopy (MS) is considered the "gold standard" for scientific analysis as it allows chemists to propose the identity of chemical that constitute the headspace of the samples which could therefore help us decipher what is triggering detector dogs' response. Additionally, the specificity for volatiles and thermal stable compounds that GC provides, makes it a suitable technique for this study. Gas chromatography theoretical basis was first describes by Martin and Synge in 1942 while GCs first application goes back 64 years, when James and Martin (79) separated fatty acid mixtures using gas- liquid partition chromatography $(79,80)$. GC has become an important tool for several applications including medical research, forensic investigations, and laboratories working on food control or environmental pollution (81). This technique is primarily used as a separation technique for the identification and quantification of volatile compounds that have boiling points up to about $350{ }^{\circ} \mathrm{C}(82)$. 


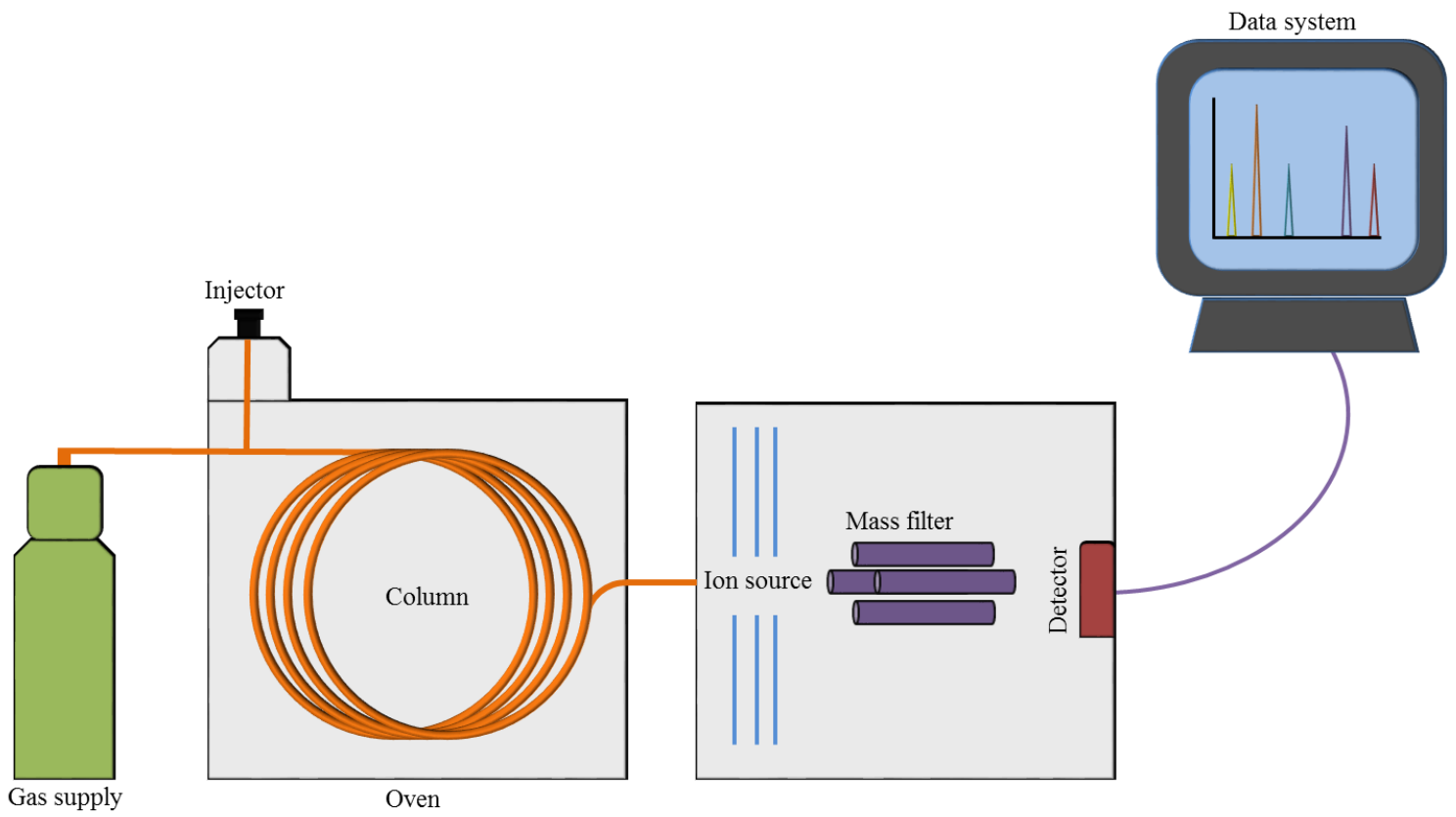

Figure 9. Gas Chromatography- Mass Spectrometer Scheme

As detailed in Figure 9, the GS-MS consists of a number of units: gas supply, sample injector, the column located in the oven, the ion source, mass filter and detector located in the MS system, and finally, the appropriate display unit (computer). With GC it is possible to sample gas, liquid or solid materials by injecting the sample into the heated injection port often by a microsyringe. Essentially, in gas chromatography there is a physical separation of sample components by means of a stationary phase and a mobile phase. When using SPME, the analytes are extracted from the sample onto the coating of the SPME, then fiber is manually injected through the heated injector port. The compounds are then thermally desorbed from the fiber, where it is evaporated in a stream of gas which allows the components to elute through the column (stationary phase) by the carrier gas (mobile phase). Is the affinity of the compounds with the stationary phase in the column what will 
dictate the velocity at which the compounds will travel through, allowing for separation and identification of the components in the mixture being analyzed. While compounds travel through the column, they get separated from each other by means of mass to charge ratio $(\mathrm{m} / \mathrm{z})$ and will further emerge as individual sample bands that can be detected and measured. The detector will provide an electrical response directly proportional to the solute as it leaves the column (83). Thus, the elution time, also known as retention time, can be measured and further used to identify the analytes. The area under the elution curve is also important as it can be used to determine the mass of the analyte eluted. Consequently, is the area under the elution curve and the retention time, the two parameters necessary for the qualitative and quantitative analysis of the compounds in the sample (83).

The carrier gas used as the mobile phase must be inert with respect to the stationary phase in the column and the components of the sample (81). Helium is the most commonly used carrier gas as it is nonreactive, nontoxic and not explosive providing good efficiency and analysis times $(84,85)$. When the sample is introduced through the injector it goes through a capillary column that can easily overloaded which could create overlapping in the chromatogram. To avoid this from happening, by automatically controlling the split valve in the injector, the sample can be splitted at a ratio selected and specified by the analyst. In other words, only part of the sample will enter the column while the rest will be discarded from the chromatograph system. What is referred as split ratio, is essentially a function of the ratio of gas flows through the GC's injector, all controlled by pressure/gas flows in the injector (86). For instance, with a split ratio of 1:10, out of 10 parts injected only one will get to the column. Since capillary columns can be easily overload the use of split injection is usually the best approach to make. But when trace analysis is been 
conducted, splitless injection is usually what is used as the volume of the samples is smaller and will risk the detection of the analyte. On the contrary, when splitless injection is used, then the sample will completely go into the column for separation. Additionally, the column must also be considered to ensure an efficient analysis as it can affect the quality of separation of the compounds in a mixture. The type, length, and diameter, of the column directly affect the basis of separation. For example, the type of column will impact the diameter of the column which in turn influences the volume of analyte that could be injected, therefore it must be adjust towards a selection that avoids compromising the chromatogram effectiveness. Moreover, the length of the column affect the retention time of the components in a mixture but instead of negatively affecting the separation of it, improves it. Finally one last consideration is the GC detector, after compounds in a mixture are separated in the column, as they leave the column they are identified by a detector, these detectors are classified in two groups: universal and selective. The universal detector could be used to detect a variety of organic compounds, while the selective detector, as the name implies, will be sensitive to a group of compound (81). The most common mass spectrometer detector associated with GC is mass selective detector (MSD). Mass selective detector provides a "fingerprint" of the molecules, i.e., its mass spectrum (Figure 10) (87). Neutral molecules from the samples will reach the spectrometer where they will be bombarded by high-energy electron beam in the ionization source to produce charged ions that could be detected. The ions formed will be further separated down a tube by an electric field, according to the $\mathrm{m} / \mathrm{z}$ ratio, and will then get to the collector where the produced signal is later recorded in a data system. The positive ions will be detected while any neutral species are undetected. 
A typical mass spectra will look like Figure 10, the $\mathrm{x}$-axis is mass to charge ratio and the $y$-axis is the abundance. As depicted in the figure the sample is limonene from which the molecular mass is indicated by the molecular peak or parent peak 136, while the base peak is the one with the greatest abundance. The percent abundance of the other peak will be relative to the base peak $(100 \%)$. The peak right next to the parent peak represents M+1 which is explained by the possibility of having a small fraction (about $1.1 \%$ ) of all carbon atoms in nature are actually the ${ }^{13} \mathrm{C}$ rather than the ${ }^{12} \mathrm{C}$ isotope (88).

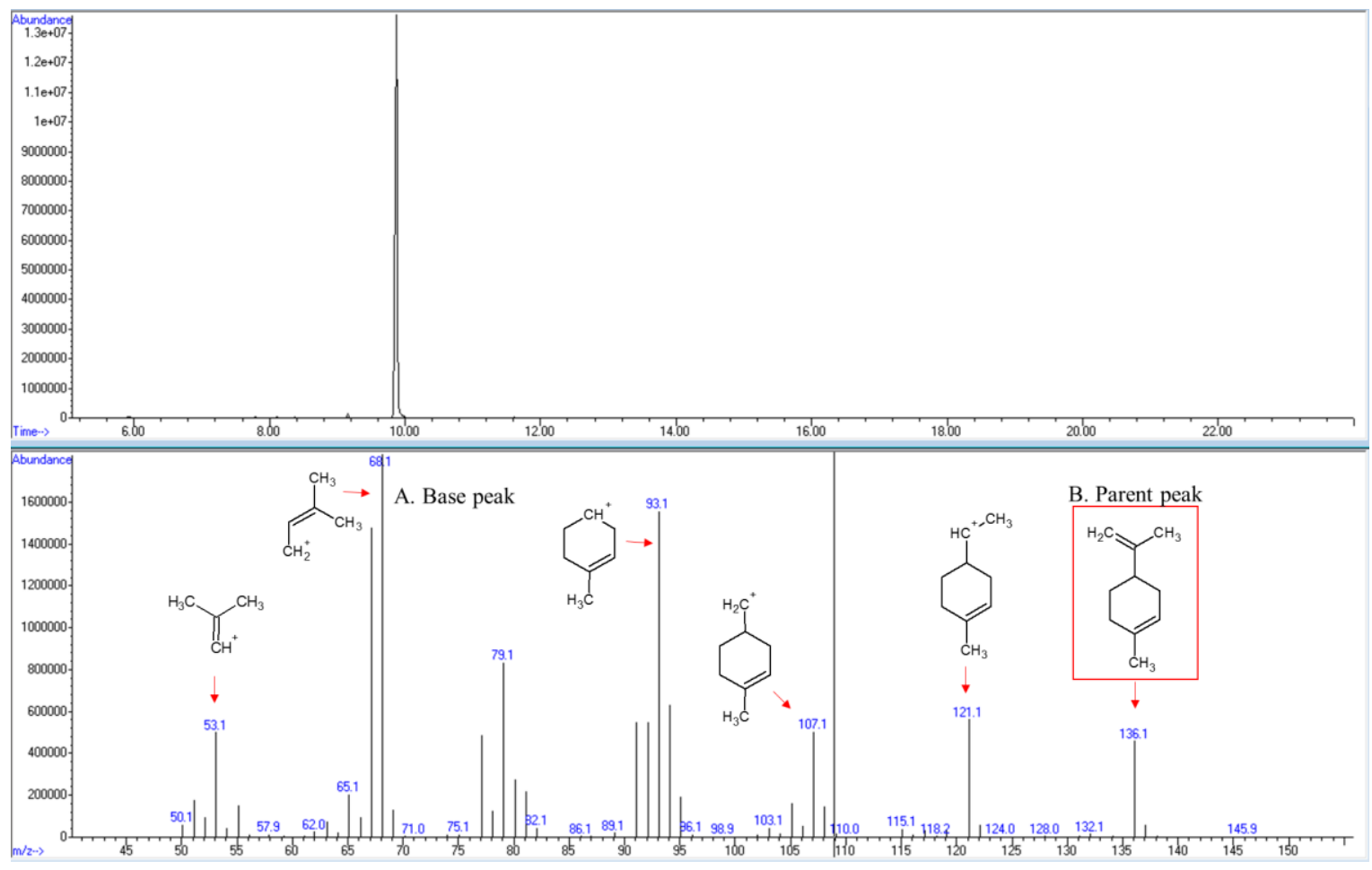

Figure 10. Mass Spectrum for Limonene A. Base Peak, B. Parent Peak 


\subsubsection{Biological Detectors}

The ability of biological detectors (such as canines) have been proven over time as they have demonstrated search, discrimination, and detection capabilities by using VOCs released by different materials or human specimens as target compounds. Different biological detectors have previously been used for a vast number of searches, for example, canaries were originally used to detect carbon dioxide in mines (89), mammals such as rats have shown to have capacity to detect explosives (90), and insects such as Drosophila melanogaster have shown ability to detect volatiles associated with illicit substances (91). Although inexpensive in price, these biological detectors have shown limitation in comparison to canines. Perhaps canaries have shown drawbacks in terms of training process, while because rats are relatively small it takes longer to search a large perimeter area. Additionally, under bad weather such as winter, most insect cannot survive, vanish or freeze to protect themselves from these conditions. However, canines are not only the most widely known biological detectors, but they are the most widely used creatures as they are able to search larger scenes for the presence of VOCs, there are not known challenges in the training process and retrieval aspects, and they are prepared to work in rain, snow, extreme heat, and difficult environments $(7,92)$.

Canines have been used for hunting and protection since ancient times. But later in history British, German and American military forces benefited from canine/soldier teams as dogs were trained for duties such as sentry, scout or patrol, messenger, or mine dog (93). However, it was not until a century ago, when fingerprints along with detection canines were recognized as detection tools to be employed by law enforcement officials (94). The

first criminal investigations where canine use was involved dates back to 1888, when the 
English used bloodhounds in an endeavor to catch a well known serial killer, Jack the Ripper. Few years later, in 1893 the state Supreme Court of Alabama (US) admitted that 'dogs may be trained to follow the tracks of a human being with considerable certainty and accuracy' (95). From this point until now, canine's search performance have be presented in court, along with other evidence, as a means to connect the perpetrator to the crime. Nowadays, they have been adapted to a variety of searches including tracking for missing persons or disaster victims $(16,96,97)$, and detection of various substances including narcotics $(98)$, explosives $(8,99)$, land mines $(96)$, and others. Nevertheless, published media such as court cases, press articles and online communications (100-102) have challenged the reliability of detector dogs due to limited reviewed research. In response to this matter, the Scientific Working Group on Dog \& Orthogonal detector Guidelines (SWGDOG) (94) was created, narrowing these concerns and scientifically validating that canines can be used to reliably detect the odors to which they are trained $(8,94,103,104)$. The training of detection canines initially involves obedience commands such as sit, stay, and search initiative. Once the canine is acclimated to the basics, the trainer will incorporate the use of a toy, which is generally a kong or a pipe, toys that will not hold any odor and is not subject to contamination. After the canine familiarizes with the toy the trainer starts developing the initiative of search by hiding the toy for the canine to search, once found it is given as a recompense. Only after this is mastered, the canine is imprinted to the odors they are supposed to detect by hiding the odors with the toy that the canine already know, if found he/she will be rewarded. Is through the association of the toy and the drugs that canines learn to distinct an illicit substance from anything else around. Once detection teams are ready, is a matter of consistency in training what will allow a reliable and highly 
effective performance (105). Therefore, SWGDOG suggests "the canine team shall spend an average of 4 hours per week in routine training to maintain the proficiency level of the team" (106). Additionally, to validate canine teams proficiency it is recommended that teams certified annually. An example is IFRI/NFSTC Detector Dog Team Certification, through this certification teams will get additional layer of credential that can be submitted as evidence that the canine team satisfactorily meet and passed all applicable standards. This certification mimic a real scenario where teams will do searches in a variety of places that can potentially be source of investigation (e.g. vehicles, a room, and/or box and luggage). Multiple amounts of real samples covering the range of drugs/explosives that canines are trained to alert, will be hidden. Teams must proof accuracy and reliability in locating their target odor by alerting at least to $90 \%$ of the hides. In court, determination of the reliability of the detection team should be based primarily on the certification records and secondarily on the regular training records (107).

Training canines for detection requires the use of aids to mimic a real search scenario; alternatives for these aids can be divided in three categories, which differ by the source where the odor is released. The first type is the actual illicit substance (i.e., marijuana, heroin, MDMA, methamphetamine or cocaine) regardless of the challenges these provide, they are the most widely used type of training aid today and are still required when certifying a detection team $(94,108)$. To obtain these aids law enforcement requires obtaining a license, strict security for storage, handling precautions, and others. Difficulties presented to obtain this type of aid have led to the development of alternatives that are easier to acquire and avoid the rest of the issues. The second type include the training aids that are designed to provide an equivalent scent by still using the actual illicit substance 
but incorporates an adsorbent material such as silica, petrolatum or cotton to which the odor is retained (108). These training aids avoid all sources of issues that the first type brings, but produces inconsistent results (109-111). The third type are known as pseudos, as they have a complete different arrangement that the original or the second category but mimic the scent of the actual illicit substance by using a compound in liquid and powder form to replicate the odor of the real material. Pseudos are the main focus of this project and have been produced using different technologies one of which is known as controlled odor permeation system (COMPS). This system is defined as a storage assembly that includes the use of a polymer bag inside a non-permeable package that will preserve the odor and will avoid contamination (112). However, the purpose of the permeable polymer bag is to control the odor that is released from the aid. An absorbent material (e.g. cellulose, alumina) is used to retain the target chemical characterizing the illicit substance and the mixture is transferred to a polymer bag that is heat-sealed. The dissipation rate desired can be manipulated by selecting different polymer bags varying in thickness while the nonpermeable package can be selected based on preservation and least contamination capacities.

Their highly sophisticated nose allows them to be a reliable operational tool for the law enforcement, as analytical instruments remain unable to replicate their effectiveness. The olfactory abilities of canines are distinguished mostly for their capability to detect target VOCs in the presence of significant background chemicals or even when hidden from view. Although is not well understood, is the canine sense of smell responsible for their high selectivity and sensitivity of their function. Research have found that the surface area of the canine olfactory epithelium is directly related to the number of receptor present, 
in general, canines have approximately 1300 genes compared with 900 in humans (113) making canines olfactory functions superior than humans' and other species. The difference in receptor number, accounts for the canines' ability to detect odors reported 10,000 to $1,000,000$ million times below the detection threshold of humans (114). For instance, Nehaus (115) compared human and dog's sensitivity to butyric acid and it was found dog's were 100,000 to $1,000,000$ times more sensible than man (115). But when it comes to compare canines different breeds, there is also variability which explains the selection of large dogs (e.g. German shepherd, Labrador) so common. Short-nosed dogs are subject to respiratory problems, albino dogs may have partial impairment of smell, while small breed dogs have less olfactory area and brain, which may reduce their capacities of olfaction (116).

The genetics behind how the smell mechanism works have been explained in different peer-reviewed articles and can be visualized in Figure 11. Olfaction begins when odorant drives into an animal's nasal cavity through the nostrils by sniffing mechanism (117). The volume of inhaled air is around $30 \mathrm{ml} / \mathrm{sec} /$ nostril equating to approximately $3.6 \mathrm{~L} / \mathrm{min}$ of sampled air (114). When searching, the dog inhales in short sharp breath at a frequency of 5-8 Hz, equivalent to $300-480$ breaths per minute $(114,117)$. The bones of the muzzle create a canal that runs all the way to the floor of the nose. This canal has olfactory cells and cells bundles that connect to the olfactory lobe located in the frontal part of the brain (116). Is via this canal that the air goes through and odorants dissolved in the mucus (surrounding the canal) are transported by receptors in direction to the lungs and smell-sensitive cells in the epithelial surface (118). The density of these smell sensitive cells (olfactory receptor cells) differs between species, and changes during the lifetime of the animal. The younger 
the animal, the more dense the cells therefore, more sense of discrimination to smell (119). Through a complex cascade of reactions in the body of the olfactory sensory neurons (OSNs), the chemical signal represented by the odorants are converted to electrical signal conveyed by the axons to the olfactory bulb in the brain (120). The fact that canines possess such a highly developed nasal cavity with much more olfactory cells in comparison to humans, makes them be considered to be one of nature's best chemical trace detectors (121).
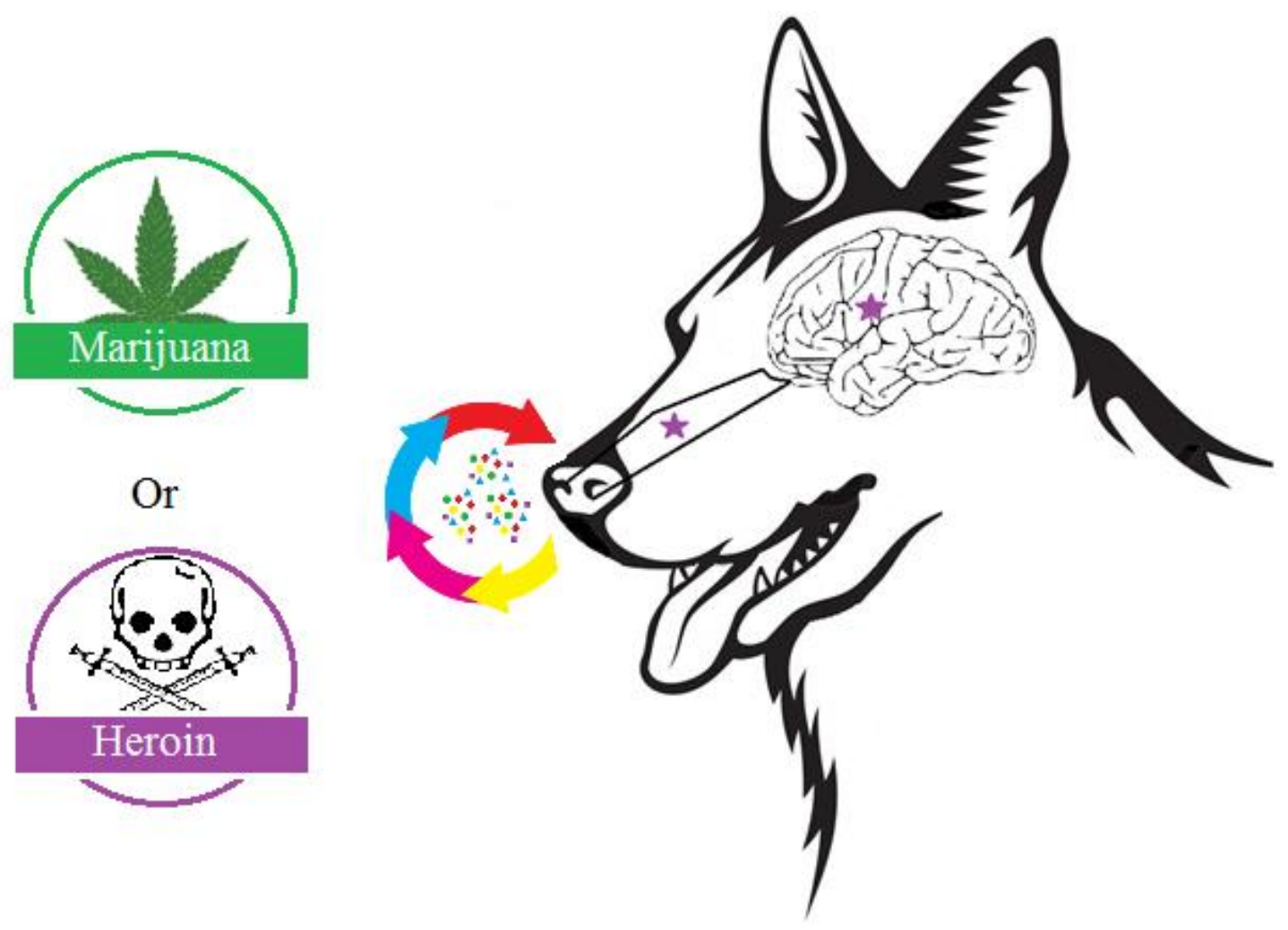

Figure 11. Canine's Olfactory System 


\subsection{Statistics}

The organization, analysis, and interpretation of the collected data were studied utilizing statistics. Universal statistical methodologies were applied to determine whether there were significantly differences among data especially when optimizing the extraction method and further when to determine occurrence of compounds in the headspace of the different illicit substances. Additionally, a compliment the results obtained from canine odor recognition tests, positive predictive (PPV) value and negative predictive values (NPV) were evaluated.

\subsubsection{ANOVA}

Sir Ronald Fisher pioneered the development of analysis of variance (ANOVA) for analyzing results of agricultural experiments (122). Today, ANOVA is one of the statistical techniques most widely used by investigators in all experimental sciences. The test is used to determine whether means and variance obtained from experimental data are significantly different from each other. To conduct this test the sums of squares and variances are considered (123). Although, ANOVA is a simple test to run, special attention is needed when selecting the appropriate ANOVA for different experimental designs, to examine whether data adhere to the modeling assumptions, and to interpret the results correctly.

One way ANOVA was performed when only one variable was been considered in an experiment where 2 or more treatments and multiple replicates were obtained. On the other hand, two-way ANOVA was performed when two variables were been considered and each variable contained different treatments. Two-way ANOVA enables one to examine

individual factors and their interactions (124). In general, ANOVA was used to determine 
any significant difference for the treatments been evaluated in the optimization process of this project.

\subsubsection{Tukey HSD}

When the data meet the assumption of homogeneity of variances (null hypothesis, $F_{c}$ $>F_{t}$ ) after running ANOVA a post-hoc must be performed to obtain a more specific result. Tukey honestly significance difference (HSD) test is considered a post-hoc test. Tukey HSD test is used to identify where in the set of data the significant difference is, as for example, ANOVA will only tell you whether there is a significant difference or not, but does not identify where the difference occurred between groups.

\subsubsection{PPV and NPV $(125,126)$}

Positive Predictive Value (PPV) and Negative Predictive Value (NPV) were calculated for the analysis of the results of the canine trials.

\subsubsection{Positive Predictive Value (PPV)}

The PPV value is the percentage of canines that alerted when in fact there was something hidden. This probability will essentially tell us how many of the alerts were correct alerts. If the number obtained is high the it suggest the training aid hidden is as good as the actual illicit substance.

$$
P P V=\frac{T P}{T P+F P}
$$

TP- True positive, FP- False positive 


\subsubsection{Negative Predictive Value (NPV)}

The NPV value is then the percentage of canines that did not alert when in fact anything was hidden. The percentage will tell us how many of the no alert were correct. If the value obtained is close to 100 then it suggest canines' successful performance.

$$
N P V=\frac{T N}{T N+F N}
$$

Equation 3

TN- True negative, FP- False negative

\section{SIGNIFICANCE OF STUDY AND RESEARCH OBJECTIVES}

The drug trade poses a threat to the United Stated national security. Since its establishment in 1973, the DEA, in coordination with other federal, state, local, and foreign law enforcement organizations has been responsible for the collection, analysis, and dissemination of drug-related intelligence (14). The System to Retrieve Information from Drug Evidence (STRIDE) provides DEA with all the information regarding the drug seizure data. For many years, marijuana and heroin are consistently among the top five drugs seized by the DEA (2014:163,295 lbs of marijuana and 2,244 lbs of heroin). These data reinforce the enormity of the problem the country faces, and the need of a tool capable to satisfy canine teams detection training and that is safe, cost effective, and easy to obtain.

Improvised explosives devises (IEDs) also posses a threat to national security. The extent and frequency of its use has created a worldwide concern and emphasized the requirement of a greater spectra of training aids that cover a wider range of currently available explosives. High sensitive methods that have potential to alleviate this issue are observed in the area of remote detection of explosives $(44,127)$. In remote detection, the 
personnel performing explosives screening maintain a safe distance from the item being screened, but the screening equipment does not (128).

The present dissertation discusses the results of laboratory and field work used to satisfy the development of new training aids, and to optimize a new method for the ease of newly developed substances canine training aids. The tasks followed to complete this project are listed below:

1. The optimization of sampling and analysis method of marijuana and heroin samples

a. Evaluation of different fiber chemistries followed by statistical analysis to support suitability of selection

b. Evaluation of SPME parameters such as extraction time, equilibrium time and temperature

2. Analysis of different illicit substances to determine the VOCs that constituting the headspace of the samples

a. Evaluation of a variety of samples provided by the law enforcement

b. Statistical analysis of data collected to determine frequency of compounds found in the headspace of all samples

3. Field trial using certifies detection canines

a. Determination of the active odorant based on frequency appearance 
i. Odor recognition test: Single compounds field evaluation

ii. Odor recognition test: Mixture of compounds field evaluation

4. Formation of controlled odor permeation system (COMPS)

a. Evaluation of different sorbent materials

b. Evaluation of different polymer bag thickness

5. Optimization of newly developed training aids

a. Instrumental analysis of odor dissipation over time

b. Odor recognition test: Proof of concept

6. Training aid alternative for explosive detection canines

a. Evaluation of IED materials

b. Optimization of collection system

c. Field trial evaluation of newly developed training aids 


\section{TASK 1. OPTIMIZATION OF SAMPLING AND ANALYSIS METHOD}

\subsection{Introduction}

The use of solid phase microextraction (SPME) as a tool for any investigation requires the optimization of the extraction technique to recover and guarantee extraction efficiency for all the analytes. To characterize the volatile organic compounds of illicit substances at a given time, headspace analysis was performed. Different parameters known to influence the effectiveness of extraction of the analytes were studied: fiber chemistry, equilibrium time, extraction time, and temperature. Selecting the appropriate SPME fiber coating for the extraction of volatiles from the headspace is of importance as it depends on the volatiles' physical and chemical characteristics, such as analyte size and polarity. This tasks aims to determine the effect of the analyte polarity and molecular size in relationship to fiber polarity and type. Thus, different fiber chemistries commercially available were used to extract different samples which results were later compared statistically. Statistical analysis was performed to select the best fiber coating on the basis of peak area, number of compounds extracted, and chemical families obtained per sample. In SPME, a small amount of the extracting phase associated with a solid support is placed in contact with the headspace of the sample for a period of time. If the time is long enough for the abundance of compounds to reach plateau, it suggests that equilibrium has been established between the sample and the extraction phase. As part of this task, the time required for the sample to reach equilibrium as well as the amount of time required for the fiber to be exposed to the headspace to achieve the best extraction or reach its maximum capacity was determined. Temperature is also a significant parameter to consider as it affects the kinetics 
of the extraction process. Thus, different extraction temperatures were also evaluated to complete the identification of the parameter that will allow an accurate response.

\subsection{Materials}

Optimization procedures were performed using an Agilent Technologies 6890N Network GC System coupled to a 5973Network MS, equipped with MSD ChemStation Software (ver 02.01.1177). Out of a number of fiber coatings commercially available, a variety of coatings was selected as to have a representation of a broad range of fiber chemistries. The selection varied in not only polarity but also mechanism of analyte uptake, and thickness.

Table 1. SPME Fiber Selection

\begin{tabular}{lllll}
\hline Fiber Coating & Thickness & Color & Abs/Ads & Polarity \\
\hline PDMS & $100 \mu \mathrm{m}$ & Red & Absorbent & Non Polar \\
PA & $85 \mu \mathrm{m}$ & White & Absorbent & Polar \\
PEG & $60 \mu \mathrm{m}$ & Purple & Absorbent & Polar \\
PDMS/DVB & $65 \mu \mathrm{m}$ & Blue & Adsorbent & Bipolar \\
DVB/CAR/PDMS & $55 / 30 \mu \mathrm{m}$ & Gray & Adsorbent & Bipolar \\
\hline
\end{tabular}

All silica fibers and manual SPME holders used for the extraction of volatiles from illicit substances were purchased from Supelco (Bellefonte, PA): Polydimethylsiloxane (PDMS, $100 \mu \mathrm{m}$ ), Polyethylene glycol (PEG, $60 \mu \mathrm{m})$, Polyacrylate (PA, $85 \mu \mathrm{m})$, Divinylbenzene/Carboxen/ Polydimethylsiloxane (DVB/CAR/PDMS, 50/30 $\mu \mathrm{m}$ ), Polydimethylsiloxane/Divinylbenzene (PDMS/DVB, $65 \mu \mathrm{m})$. For each extraction time and equilibrium time analysis, samples were sealed in $10 \mathrm{~mL}$ screw-top clear vials with 
polypropylene hole caps and PTFE/silicone septa (Supelco, Bellefonte, PA). When conducting the temperature study, samples were placed in a Isotemp Digital Dry Baths/ Block Heater (Fisher Scientific, Pittsburg, PA).

\subsection{Methods}

The extraction efficiency obtained with the SPME method can be affected by the fiber type, extraction time and temperature, and equilibrium time. Before the evaluation of the best extraction method to analyze the headspace of the different samples, a comprehensive literature search was performed. A variety of published articles were evaluated in regards to GC/MS methods for the analysis of headspace of both heroin and marijuana samples. Although the objective of the researchers in the past has not been the same as the one previously stated, the GC/MS have certainly been used to evaluate these substances.

\subsubsection{Extraction Method}

\section{Marijuana}

Samples of marijuana were obtained through law enforcement personnel registered under the control substance act of 1970 through DEA. This permit allows individuals to possess narcotics for a variety of listed purposes. Marijuana samples are usually evidence that can further be destroyed but instead are evaluated and classified in the laboratory to be potentially used for research, canine training, pharmacies, teaching institution, among others. All marijuana samples consisted of an uniformly physical characteristics, dried, green, and plant alike. An average of $0.500 \mathrm{~g}$ was weight into a brand new $10 \mathrm{~mL}$ screwtop clear vial. Samples were only allowed to be tested for no more than $2 \mathrm{hrs}$. Therefore, the time limits evaluated were $15 \mathrm{~min}$ from each other up to $90 \mathrm{~min}$. To evaluate the factors 
influencing the HS-SPME such as fiber chemistry, equilibrium and extraction time, a Fisher Scientific ${ }^{\mathrm{TM}}$ Big-Digit Time/Stopwatch was used to keep track of the analysis time. Samples were tested in triplicates and a blank was included for control purposes. To maintain a record of the environmental conditions before each set of samples was extracted, temperature and humidity were recorded using a HHF81 4-in-1 anemometer, hygrometer, light meter and thermometer (Omega Engineering, Inc., Stamford, CT, USA). Prior to any extraction, new fibers were conditioned according to the manufacturer's instructions (Table 2) by inserting them into the GC injector port at the recommended temperature and for the indicated amount of time depending on the fiber chemistry. An extraction procedure was established with the initial conditions equilibrium time of one hour. Upon completion, SPME fibers were injected through the septa in the vial hold in place using a clamp. The SPME fibers were exposed to the headspace of the samples by pushing the plunger and exposing the fiber to volatiles in the headspace for an hour (time randomly selected to keep uniformity through the analysis).

Table 2. Temperature and Conditioning Recommendations for GC

\begin{tabular}{lll}
\hline Fiber Coating & Conditioning Temp & Time (hr) \\
\hline PDMS & $250{ }^{\circ} \mathrm{C}$ & 0.5 \\
PA & $300^{\circ} \mathrm{C}$ & 2 \\
PDMS/DVB & $250{ }^{\circ} \mathrm{C}$ & 0.5 \\
DVB/CAR/PDMS & $270{ }^{\circ} \mathrm{C}$ & 1 \\
\hline
\end{tabular}

After selecting the most suitable fiber coating for the extraction technique, variables known to influence the extraction of VOCs were evaluated; extraction time, equilibrium time and temperature. These factors can easily be controlled and optimized to obtain the 
highest possible extraction efficiency. For instance, while different extraction time (independent variable) were analyzed, other factors were held constant (controlled variables). The experimental design first consisted on evaluating the best extraction technique. Different extraction times were evaluated: 15, 30, 45, 60 and 90 mins. An average of $0.500 \mathrm{~g}$ was weight into a brand new $10 \mathrm{~mL}$ screw-top clear vial and immediately after, labeled triplicate samples and blank vials were sealed. A controlled equilibrium time of 30 min was selected for the study. The SPME fibers were injected through the septa of the vials and hold in place using a clamp attached to a clamp holder after the $30 \mathrm{~min}$.

Triplicate samples and a blank were extracted for different times, previously mentioned. Upon completion, analysis of variance (ANOVA) was used to evaluate statistical significant difference between the number of compounds extracted, the peak area (abundance), and chemical families of the volatiles extracted depending on time.

Once the most suitable equilibrium time was determined, it was used as a controlled variable while different extraction times were evaluated: 15, 30, 45, 60 and 90 mins. An average of $0.500 \mathrm{~g}$ was weight into a brand new $10 \mathrm{~mL}$ screw-top clear vial and immediately after, labeled triplicate samples and blank vials were sealed. Samples were equilibrates for five different time periods. The SPME fibers were injected through the septa of the vials and hold in place using a clamp attached to a clamp holder for the time determined to be suitable for the extraction of VOCs.

\section{Heroin}

Heroin samples were also obtained through law enforcement personnel registered under the control substance act of 1970 through DEA. All heroin samples consisted of 
equal physical characteristics: powdered, brown/tan color. An average of $0.230 \mathrm{~g}$ was weight and transferred to a brand new $10 \mathrm{~mL}$ screw-top clear vial. Samples were tested in triplicates and a blank was included for control purposes. Time and environmental conditions such as temperature and humidity were recorded. Prior to perform any extraction, the fibers were conditioned according to the manufacturer's instructions (Table 2) by inserting them into the GC injector port at the recommended temperature for as much time as was suggested. An extraction procedure was established with the initial conditions equilibrium time of one hour. Upon completion, SPME fibers were injected through the septa in the vial hold in place using a clamp. SPME fibers were exposed to the headspace of the samples by pushing the plunger and exposing the fiber to volatiles in the headspace for an hour (time randomly selected to keep uniformity through the analysis).

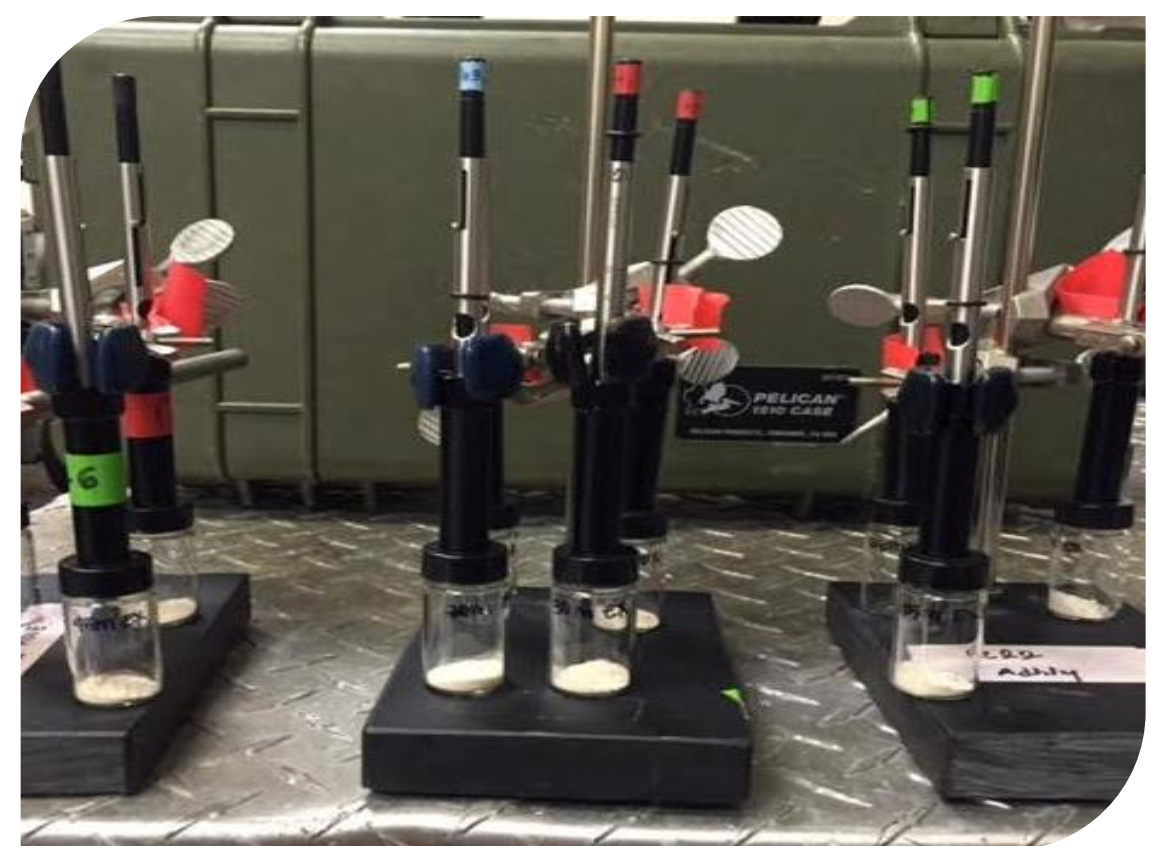

Figure 12. Triplicate of Heroin Samples and Blank Extraction Using SPME 
The equilibrium study and extraction study were performed once the most suitable fiber chemistry was determined. Different equilibrium times were evaluated: 15, 30, 45, 60 and 90 mins. An average of $0.230 \mathrm{~g}$ was weight into a brand new $10 \mathrm{~mL}$ screw-top clear vial and immediately after, labeled triplicate samples and blank vials were sealed. Once samples were let equilibrate for different amount of times, $30 \mathrm{~min}$ extractions were performed (controlled variable). Extractions were performed by injecting the SPME fiber through the septa of the vials and hold in place using a clamp attached to a clamp holder for the time been studied. Once the equilibrium time was determined, the same process was performed to determine a suitable extraction time.

\subsubsection{GC/MS Method}

The GC used in the experiment was equipped with a temperature programmable inlet (TPI) injector. The GC was fitted with a $30 \mathrm{~m}$ x $0.250 \mathrm{~mm}$ i.d. HP-5MS column coated with a $0.25 \mu \mathrm{m}$ film of stationary phase (Agilent Technologies, Santa Clara, CA, USA). The injector was equipped with a $2 \mathrm{~mm}$ i.d. liner (Supelco, Bellefonte, PA, USA) (in order to obtain better peak shapes) and was operated in splitless/split mode with a splitless time of $1 \mathrm{~min}$.

\section{Marijuana}

The column temperature was held at $40^{\circ} \mathrm{C}$ for $3 \mathrm{~min}$ after the injection, then programmed at $10^{\circ} \mathrm{C} / \mathrm{min}$ to $250^{\circ} \mathrm{C}$. Helium was used as a carrier gas at a constant linear velocity of $35 \mathrm{~cm} / \mathrm{s}$. To determine the concentration of the compounds in the sample a set of known concentration standards was analyze to further create a standard calibration

curve. To analyze the series of standards across a range of concentrations (5 ppm- 100 
ppm) an automatic liquid sampler (ALS) method was created. The GC-MS data acquisition started after the elution (solvent delay $5 \mathrm{~min}$ ) of methylene chloride $\left(\mathrm{MeCl}_{2}\right)$ (used solvent). To avoid any source of contamination and/or carry over, syringe was pre-watched twice before $1 \mu \mathrm{L}$ liquid standard was injected.

\section{Heroin}

The column temperature was held at $45^{\circ} \mathrm{C}$ for $5 \mathrm{~min}$ after the injection, then programmed at $15^{\circ} \mathrm{C} / \mathrm{min}$ to $130^{\circ} \mathrm{C}$ where it was held for $5 \mathrm{~min}$. After that temperature increased $25^{\circ} \mathrm{C} / \mathrm{min}$ to $160^{\circ} \mathrm{C}$, where it was held for $0.5 \mathrm{~min}$ and later kept increasing $25^{\circ} \mathrm{C} / \mathrm{min}$ to its final temperature $250^{\circ} \mathrm{C}$. Helium was used as a carrier gas at a constant linear velocity of $34 \mathrm{~cm} / \mathrm{s}$. To determine the concentration of the compounds in the sample a set of known concentration standards was analyze to further create a standard calibration curve. To analyze the series of standards across a range of concentrations (200 ppm- 375 ppm) an automatic liquid sampler (ALS) method was created. The GC-MS data acquisition started after the elution (solvent delay $1.80 \mathrm{~min}$ ) of acetonitrile (ACN) (used solvent). To avoid any source of contamination and/or carry over, syringe was pre-watched twice before $1 \mu \mathrm{L}$ liquid standard was injected.

\subsection{Results and Discussion}

Different fiber chemistries ranging from adsorbent to absorbent coatings, polar to nonpolar in a range of coating thickness were analyzed to determine the best coating for the extraction of analytes under study. There are 4 major criteria commonly considered when selecting the proper fiber coating. These are molecular weight/size of an analyte, the polarity, the analyte concentration level, and the complexity of the sample matrix (129). In 
this task, the analysis was focused on the weight of the analytes as well as the polarity associated to chemical families in relation with the fiber coating. The molecular weight of an analyte determines how rapidly it can move in and out of the fiber phase coating and through the sample (129). These two variables (weight and speed) are said to be inversely proportional. For instance, the smaller the analyte is, the faster it travels, making challenging the retention of it. However, the larger the analyte is, the longer it takes for it to move, thus the longer it takes for it to reach equilibrium. Polarity also plays an important role in this matter. The selection of a suitable fiber will also depend on the polarity of the compound(s) of interest. Ideally, if the analyte is polar, you will use a polar fiber to be more selective, not only to extract polar analytes but also to repel non-polar analytes.

\subsubsection{Marijuana}

\subsubsection{Fiber chemistry}

Marijuana is a naturally harvested product, which makes the process of characterizing the headspace a challenge as there is a dramatic variation of compounds from plant to plant. Therefore, the study of the variation of volatiles composition of various marijuana samples must be assessed, as there is little information on it. Previous research have suggested that the chemicals responsible for the odor released from marijuana are known as terpenes, which are generally non polar and often volatile. However, if only a non-polar fiber is considered then the information obtained may be limited. It is important that we also to consider the fact that in nature, terpenes occur predominately as hydrocarbons, alcohols and their glycosides, ethers, aldehydes, ketones, carboxylic acids and esters. For that 
reason, it is not ideal to use a selective fiber but rather one that would extract the greatest amount of compounds, considering both polar and non-polar volatiles. 


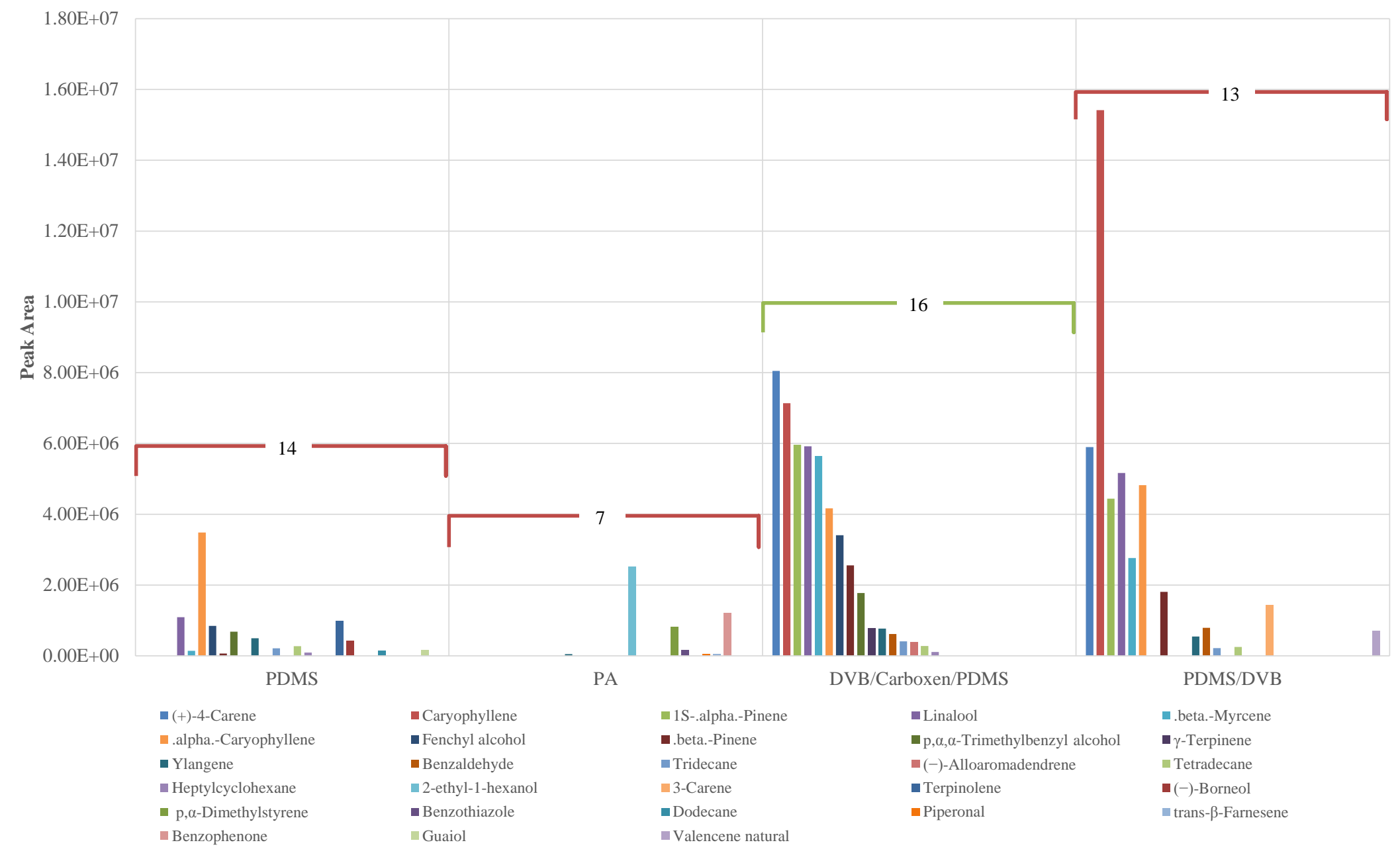

Figure 13. Abundance and No. of VOCs Found in Marijuana Headspace Samples Using Different Fiber Chemistries 
The responses for the fiber coatings yielding the highest and lowest responses have been determined on the basis of the peak area (abundance), the response was complemented with the number of compounds extracted per fiber and chemical families extracted over time. A total of 28 compounds were consistently found in at least $66.6 \%$ of the samples while extracting using different SPME fibers varying in polarity and sorbent process. However, it is important to know that no all compounds were detected in all fibers and conditions. The compounds that were detected belong to the following families: terpenes (18 compounds), alkanes (4 compounds), ketones ( 1 compound), alcohols (52), and aromatics (3 compounds).

The DVB/CAR/PDMS fiber extracted the highest amount followed by PDMS/DVB while the PDMS and PA fibers were least efficient in terms of sample diversity. When samples were extracted using PA less than $50 \%$ of the compounds extracted with DVB/CAR/PDMS were extracted while 13\% difference in extracted compounds was found between PDMS and DVB/CAR/PDMS as they have a more similar chemistry that PA. Additionally, as depicted on the Table 3 the extracted compounds focused on the terpines with over $70 \%$ of the total compounds and $90 \%$ of the total peak area. The present of terpenes was expected as it has been previously reported that this chemical family predominates in the headspace of plants. The relative percentage of the different chemical families extracted using DVB/CAR/PDMS and PDMS/DVB is shown in Table 3. Both fibers favored the same chemical families except for alcohols; terpenes, alkanes, and aromatic. 
Table 3. Percentage of Total Peak Area of Each Chemical Family

\begin{tabular}{lcccc}
\hline & PDMS & PA & DVB/CAR/PDMS & PDMS/DVB \\
\hline Terpenes & 84.57 & 19.01 & 93.44 & 97.08 \\
Alkanes & 7.92 & 0.00 & 1.64 & 1.09 \\
Ketones & 0.00 & 24.77 & 0.00 & 0.00 \\
Alcohols & 7.51 & 51.85 & 3.65 & 0.00 \\
Aromatics & 0.00 & 4.38 & 1.27 & 1.83 \\
\hline
\end{tabular}

The slight difference between those compounds extracted with DVB/CAR/PDMS but not with PDMS/DVB can be explained by the additional polymer coating included in DVB/CAR/PDMS; carboxen. Carboxen allows for a broader spectrum of boiling points as low boiling point compounds can be extracted while DVB-PDMS layer allows the extraction of those with high boiling points leading the extraction of a greater amount of compounds. Over $60 \%$ of the compounds have boiling point higher than $165^{\circ} \mathrm{C}$ while $40 \%$ have boiling point that goes as low as $64{ }^{\circ} \mathrm{C}$ a compound that was extracted by DVB/CAR/PDMS and not PDMS/DVB. ANOVA showed no significant difference between DVB/CAR/PDMS and PDMS/DVB however, DVB/CAR/PDMS was selected to be used as it provides with a good representation of the volatile profile and it satisfactorily extracts compounds previously highlighted in other studies $(75,130)$.

\subsubsection{Extraction}

Extraction time 15, 30, 45, 60 and 90 minutes were evaluated to determine the time where both number of compounds and abundance (peak area) of peaks was favorable to obtain a represerntative profile of volatiles in marijuana headspace. 
The number of compounds extracted was different for all the extraction times that were analyzed except for 60 and 90 minutes which, were also the extraction times that greater amount of compounds were extracted. To determine whether there was or not significant difference between the abundance of those compounds that were commonly extracted ANOVA was performed. The comparison of 60 and 90 minutes showed no significant different in number of compounds nor abundance of the peaks. Greater than $60 \%$ of the compounds' peak area have stayed or decrease in abundance while the rest have increased but not significantly. The fact that peak area does not significantly change after 60 minutes is indicative of compounds that have reached equilibrium therefore do not increase in abundance as the amount of analyte extracted onto the fiber coating is at a maximum. The peak areas were represented in a line graph and it reflected the behavior of how the abundance changed overtime. As depicted after 60 minutes, the peak area (abundance) reached plateau which is indicative that equilibrium have been reached. 


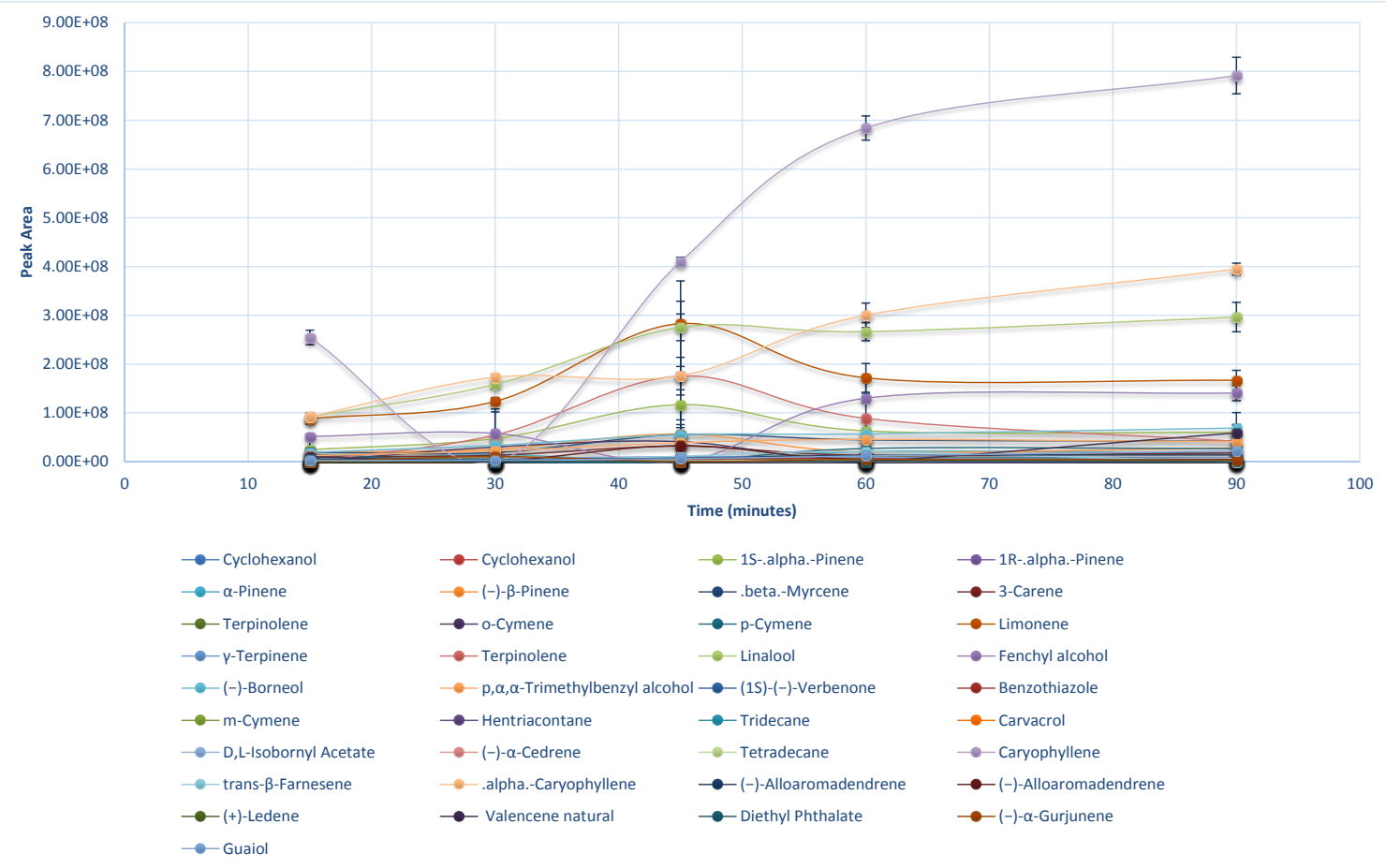

\section{Figure 14. Abundance of 15, 30, 45, 60, and 90 Extraction Time of $0.5 \mathrm{~g}$ of Marijuana Using SPME}

Upon completion of the analysis, it was concluded there was no significant difference between 60 and 90 minutes equilibrium time $(\mathrm{F}=0.035<\mathrm{Fcrit}=4.06)$.

\subsubsection{Equilibrium}

Different equilibrium times were studied to determine which was the most feasible to obtain a representative volatile profile for marijuana samples. Starting from 15 minutes, additional times were evaluated varying in 15 from each other for up to 60 minutes. The reason for the limit to be 60 minutes is due to restrictions for sampling. When samples were equilibrated for various times individually, the number of compounds obtained was different from each other. The greatest amount of compound was obtained in 15 and 45 minutes. Out of those compounds in common, $35 \%$ of the compounds increased in 
abundance while greater than $60 \%$ of the compounds either stayed or decreased in abundance indicating that the majority have reached equilibrium by 15 minutes as the abundance have reached the maximum capacity onto the fiber.

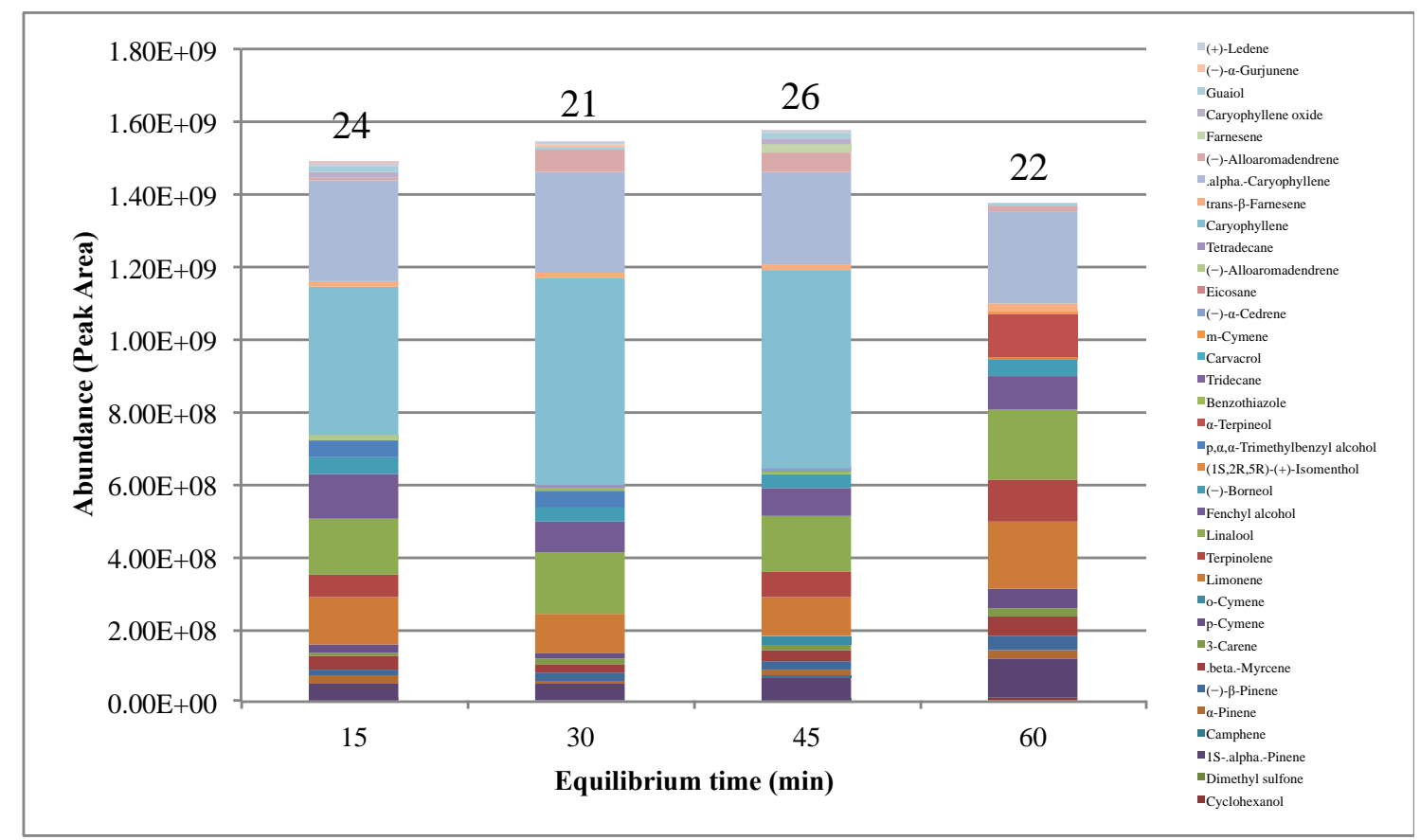

Figure 15. Abundance of 15, 30, 45 and 60 Equilibrium Time of $0.50 \mathrm{~g}$ of Marijuana Followed by 60 min Extraction Using SPME

\subsubsection{Temperature study}

After analyzing triplicates of a marijuana sample, the number and abundance of compounds collected was greater for those extractions that were performed at higher temperatures. Although higher abundances were obtained when extraction were performed at $50{ }^{\circ} \mathrm{C}, 30 \%$ of terpenes (7/23 total terpenes found in the sample used for optimization) were being compromised. Based on previous research (Hood, et al., Lai, et al.) terpenes are 
likely to be responsible for marijuana odor thus, the effectiveness of these compounds' extraction must be considered for further analysis.

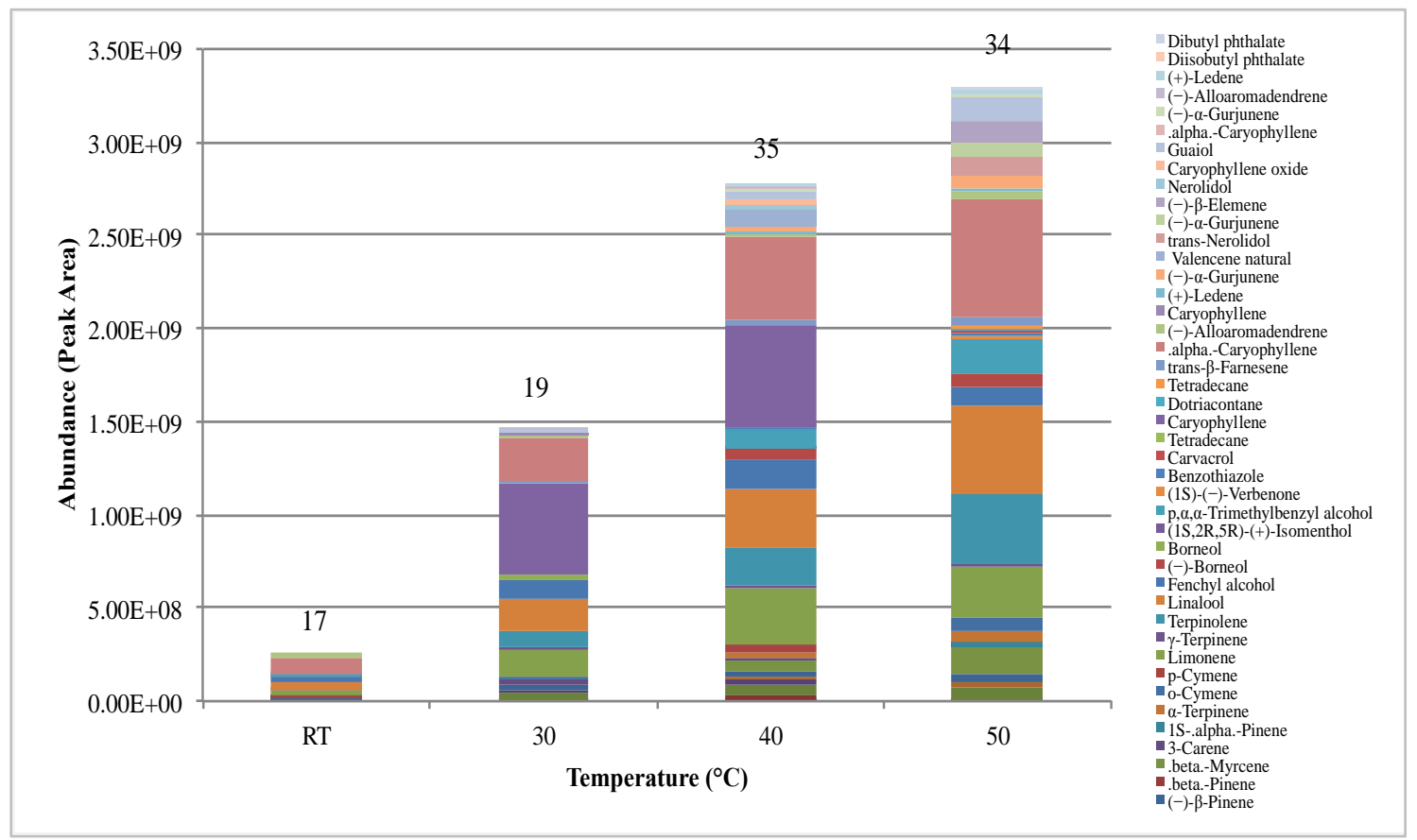

Figure 16. Abundance and No. of Compounds of $0.5 \mathrm{~g}$ Marijuana Samples Extracted at Different Temperatures, $\mathrm{RT}, 30{ }^{\circ} \mathrm{C}, 40{ }^{\circ} \mathrm{C}, 50^{\circ} \mathrm{C}$

\subsubsection{Heroin}

Based on articles that have been published (9), it is known that the major volatile found in the headspace of this illicit substance is acetic acid, a polar compound that's found in a variety of comestible products. However, the fiber selection could not be focused only on getting the best of this compound but rather in selecting a coating capable to extract the greatest amount of compounds and peak area (abundance) representative of the headspace of the sample. 


\subsubsection{Fiber chemistry}

A variety of fiber chemistry of diverse polarity and sorption (adsoption/absorption) process were assayed in order to evaluate their effect on heroin volatiles compounds extracted by SPME. A total of 12 compounds were tentatively identified in this study, although not all of them were detected with all fiber and conditions tested. Identified compounds belonged to the following chemical families: acids (52), amides (1 compounds), ketones (1 compound), alcohols (1 compound), alkanes (3 compounds), isocyanate (1 compound).

PDMS/DVB extracted the highest total amount of volatile compounds followed by DVB/CAR/PDMS. The total amount of compounds extracted with PEG and PA was lower. More specifically, when samples were extracted using PEG and PA, they extracted 45 and $22 \%$ less of the amount extracted with PDMS/DVB under the same extraction conditions. Additionally, the extracted compounds focused on acids and amides, 80 and 20\% respectively for PEG, and 57 and $14 \%$ respectively for PA, rather than giving a broad spectrum of chemical families. Therefore, absorbent/polar fibers were discarded for further detailed consideration about individual compounds due the specificity of chemical families being excluded from extraction leading to lower amount of compounds being extracted in comparison to adsorbent/bipolar fibers.

The relative percentages of the different chemical families extracted by DVB/CAR/PDMS and PDMS/DVB is shown in the table below. Both fiber chemistries favored the same chemical families; acids, amides, ketones, alcohols, and alkanes. Although PDMS/DVB showed higher proportionality for ketones, alcohols and alkanes is was not significantly different from what DVB/CAR/PDMS was able to extract. 
Table 4. Proportionality (\%) of Peak Area per Chemical Family

\begin{tabular}{lcccc}
\hline & PEG & PA & DVB/CAR/PDMS & PDMS/DVB \\
\hline Acids & 96.62 & 94.47 & 88.41 & 87.48 \\
Amides & 3.38 & 4.28 & 6.85 & 1.99 \\
Ketones & 0.00 & 0.00 & 0.75 & 1.10 \\
Alcohol & 0.00 & 0.58 & 3.00 & 4.78 \\
Alkanes & 0.00 & 0.00 & 0.99 & 4.65 \\
Isocyanate & 0.00 & 0.66 & 0.00 & 0.00 \\
\hline
\end{tabular}

Garcia-Esteban et. al. stated that carboxen is a porous carbon with a high surface area (around $1200 \mathrm{~m}^{2} \mathrm{~g}^{-1}$ ) which has been described as adequate for extraction of low boiling point compounds, although it allows the adsorption of a wide range of compounds due to the presence of different types of pores (micro-, meso-, and macropores). The fact that DVB/CAR/PDMS has a DVB-PDMS layer allows this fiber to also extract high boiling point compounds. However, the boiling point of the compounds extracted ranged between $112-287{ }^{\circ} \mathrm{C}$, considered to be high boiling points what can explain the aspect of no significant difference been found when comparing number and abundance (peak area) when using PDMS/DVB and DVB/CAR/PDMS. Thus, both fibers tested, PDMS/DVB and DVB/CAR/PDMS, provides a similar volatile compounds profile for heroin, and both satisfactorily extracts compounds previously highlighted in other studies.

\subsubsection{Extraction}

In practice, one of the variables affecting the migration of compounds into the headspace is the time sample is let sit to reach equilibrium, if the contents inside the sample vial are left long enough, the relative concentrations of a compound between the two phases 
(sample and headspace) will reach a steady value (or equilibrium) (131). To determine the suitable amount of time the sample must be left sit to reach equilibrium before the extraction, an equilibrium test was performed. To evaluate how the equilibrium time will influence the extraction of the samples a controlled variable research design was followed. One variable (equilibrium time) known as the independent variable was manipulated, while the dependent variable was kept constant.

Extraction time 15, 30, 45, 60 and 90 minutes were studied to determine the most suitable parameter to extract volatiles from heroin. Studied times were chosen for practical purposes, in order to identify the shorter extraction time giving rise to a representative volatile profile of heroin within the range of time assigned to conduct the sampling.

Except for two compounds (acetic acid and 2-ethyl-1-hexanol) the volatiles extracted from heroin samples were not consistent. Although the number of compounds did not follow a trend over time, for the most part 30, 60 and 90 minutes shared similar numbers of extracted compounds (no significantly different). The reason for such a variety of numbers could be explain by the difference in volatility and affinities for the fiber coating. Those compounds with longer equilibration time should increase their proportion with longer extraction e.g., phosphates. However, those achieving equilibrium in shorter times, rather than increase in abundance, will decrease with longer extraction time, and therefore, decrease in proportion e.g., acetic acid.

Peak area is also a variable been considered in this study. When ANOVA was performed to determine whether there was significant difference between those common compounds that were consistently extracted no significant difference was encountered. 
Therefore, for time convenience purposes, 30 minutes extraction was concluded to be feasible for studying volatiles expelled from heroin samples.

\subsubsection{Equilibrium}

After analyzing triplicates of samples provided by a licensed source it was noticeable that the compounds extracted per sample were not consistent at any point except for acetic acid. Therefore, acetic acid was the compound selected as representative of those volatiles in the headspace of heroin. Five different equilibrium times were evaluated, each increasing consecutively by 15 minutes. The time at which the area under the curve becomes constant or no longer increases was considered to be the equilibration time. The graph of abundance (peak area) suggests that adsorption reached equilibrium after 60 minutes of exposure to DVB/CAR/PDMS. Although clear to the naked eye, analysis of variance (ANOVA) was performed to determine whether or not there was any significant different between the equilibrium times tested. In fact, there was a difference. Therefore, Tukey's HSD test was performed and the difference was established to be located between 60 and 90 minutes where is shown as a significant drop in the graph. 


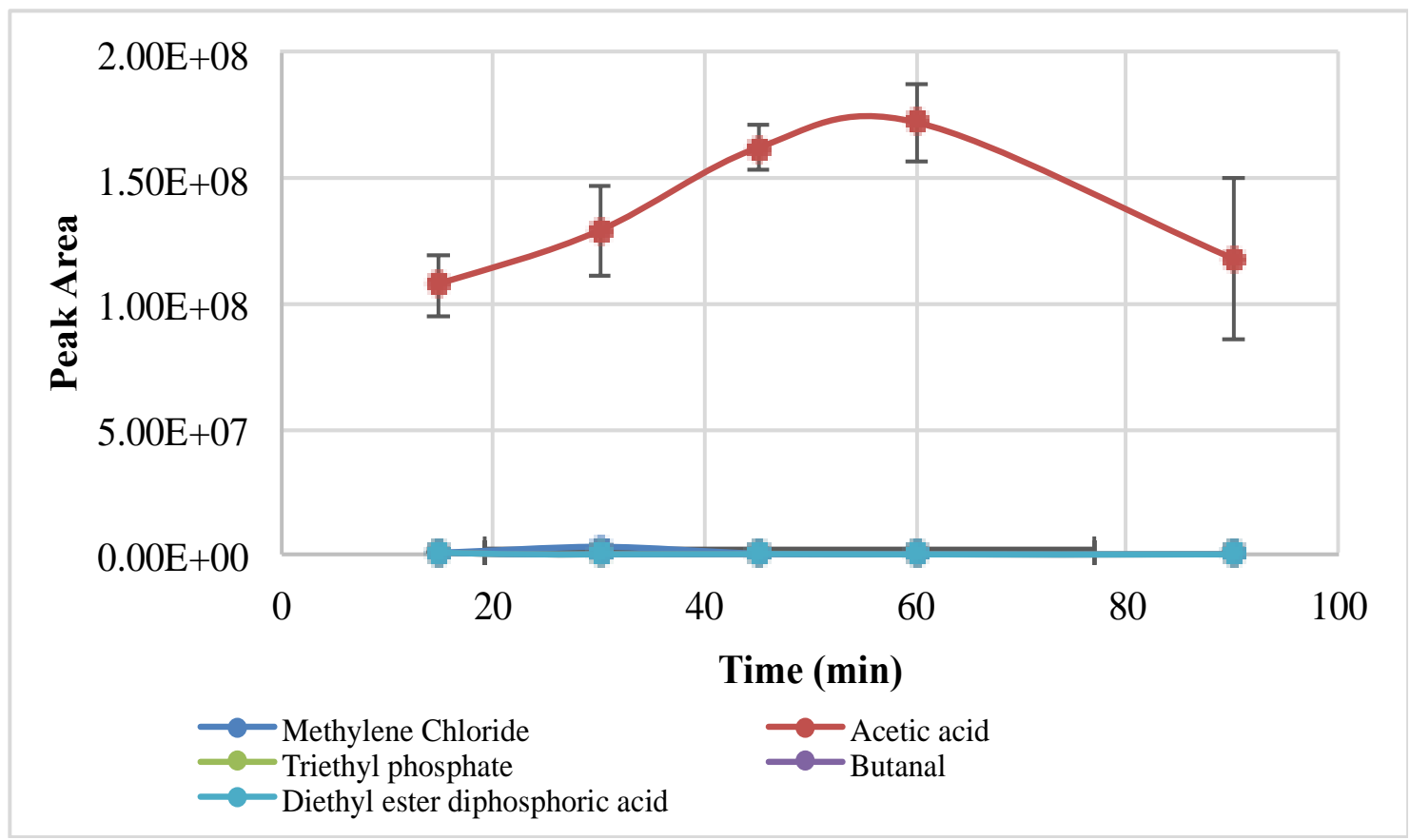

Figure 17. Abundance of a 15, 30, 45, $90 \mathrm{~min}$ SPME Equilibrium of $0.50 \mathrm{~g}$ of Heroin Followed by 30 min Extraction

\subsection{Conclusion}

Parameters affecting the recovery of VOCs from illicit substances (132) using SPME were investigated. The goal was to optimize SPME experimental procedure so as to obtain high extraction conditions within a reasonable time. The SPME method has been successfully developed to extract VOCs from marijuana and heroin samples. Coupled with GC-MS, this SPME method showed high sensitivity and satisfactory reproducibility.

The SPME technique enables a simple and rapid measurement, which would be applied for quantification of VOCs from different illicit substances. Different parameters (fiber chemistry, extraction time, equilibrium time, and temperature) known to affect the VOCs recovery from unknown samples were investigated. Additionally, significant 
difference among different conditions was determined by conducting ANOVA, if significant difference was encountered, then Tukey's HSD test was performed.

Fiber chemistries varying in polarity and thickness were evaluated to identify the fiber with the potential of characterizing the VOCs present in the headspace of marijuana and heroin samples. Number of compounds, abundance and chemistry families extracted were considered to select the best. The 30/50- $\mu \mathrm{m}$ DVB/CAR/PDMS fiber gave the best combined sensitivity to establish the VOC makeup of both illicit substances headspace. Most important experimental parameters (speed, sensitivity, accuracy, and precision) are determined largely during extraction. Therefore, different extraction times were evaluated from which 60 and 30 min for marijuana and heroin respectively were considered as the most suitable considering, number of compounds and variety of chemical families extracted at a reasonable time. Equilibrium time; the time after which the amount of analyte extracted remains constant, have also being considered in this study (12). After completing a throughout analysis, 15 and $45 \mathrm{~min}$ were selected as suitable time for samples of marijuana and heroin to reach equilibrium. Additionally, temperature is another parameter that can affect the extraction of the analyte in HS-SPME. By raising temperature, the partition constant of the analyte is increased in favor of the gaseous sample; therefore, partitioning of the analytes between the sample and headspace can reach equilibrium more quickly (133). Different temperatures were evaluated, including room temperature, $30^{\circ} \mathrm{C}$, $40{ }^{\circ} \mathrm{C}$ and $50{ }^{\circ} \mathrm{C}$. While increments in temperature were in fact improving the number of compounds been extracted at higher temperatures terpenes were been comprised which lead the selection of $40{ }^{\circ} \mathrm{C}$. 


\section{TASK 2. DETERMINATION OF VOCS FROM ILLICIT SUBSTANCES}

\subsection{Introduction}

The odor associated with the alert by detector canines, are suspected to be VOCs expelled by the substance rather than the drug itself $(134,135)$. For instance, in the case of cocaine, MDMA and methamphetamine, field test simulating real search scenarios have been conducted and have demonstrated the effectiveness of detector canines alert to chemical compounds identified as the dominant odor; methyl benzoate, piperonal, and benzaldehyde respectively $(5,134)$. However, the idea of one active odor per drug cannot be generalize, it is not known whether it is one chemical, several, or the entire VOC profile is what establishes the canine's olfactory "match" that causes an alert response (135). Furthermore, although theories suggest that canines alert to the most volatile chemical in the headspace, to date, there are not peer-reviewed studies to support this theory. In fact, previous laboratory studies on heroin training development suggested the most volatile compound in heroin headspace as potential training aid, but to no success, as it was revealed that it contained an odor similar to that of vinegar, acetic acid found in many comestible products. Therefore, the first step towards the development of training aids for detection canines is to characterize the VOCs present in the headspace of marijuana and heroin samples. Although headspace of both substances have been previously analyzed $(130,136)$, developing training aids to achieve an universal odor chemical profile requires a better understanding of the VOCs associated with the samples. It is known that illicit substances in general expel many VOCs, but a different approach needs to be considered in order to narrow down the number of compounds found in the headspace in an effort to determine the volatile(s) required to develop a training aid for detection canine. Therefore, 
chemical families and volatiles frequency of occurrence were identified as potential compounds to be used as training aid. The optimized SPME/GC-MS methods for the headspace analysis of marijuana and heroin samples respectively were used to achieve this goal.

\subsection{Materials}

Samples of marijuana and heroin were obtained through law enforcement personnel registered under the control substance act of 1970 through DEA. This act allows individuals to possess narcotics for a variety of listed purposes including canine training. Samples were allowed to be analyzed for a maximum of $2 \mathrm{hr}$, time frame considered when optimization was performed.

Samples and standards

\section{Marijuana}

Individual terpenes ( $\alpha$-pinene, $\beta$-pinene, 3 -carene, limonene, linalool, fenchyl alcohol, borneol, $\beta$-caryophellene, alloaromadendrene and caryophellene oxide) were obtained from Sigma (Sigma-Aldrich, Co., St. Louis, MO, USA). A single standard of the eleven terpenes was prepared in Fisher Scientific Optima ${ }^{\mathrm{TM}}$ methylene chloride (Fisher Scientific, Fair Lawn, NJ, USA) and was used for this study as a primary stock solution at concentrations ranging from 5-100 ppm. Working standards were prepared by serial dilutions in $5 \mathrm{ml}$ PYREX ${ }^{\mathrm{TM}}$ EZ Access ${ }^{\mathrm{TM}}$ Class A volumetric flasks (Fisher Scientific, Fair Lawn, NJ, USA). 


\section{Table 5. Marijuana Standards Serial Dilutions}

\begin{tabular}{ll}
\hline $\mathrm{ppm}$ & Stock solution $(\mu \mathrm{L})$ \\
\hline 5 & 2.5 \\
10 & 5 \\
25 & 12.5 \\
50 & 25 \\
75 & 37.5 \\
100 & 50
\end{tabular}

*After stock solution aliquot was added to the volumetric flask, solvent (methylene chloride) was used to complete up to the mark

\section{Heroin}

Acetic acid was obtained from Fisher (Fisher Scientific, Fair Lawn, NJ, USA) and was 99.7\% pure. A stock solution was prepared in Fisher HPLC grade acetonitrile $99.9 \%$ pure (Fisher Scientific, Fair Lawn, NJ, USA) and was used for this study as a primary stock solution at the concentrations ranging from 200-375 ppm. Working standards were prepared by serial dilutions in $5 \mathrm{ml}$ PYREX ${ }^{\mathrm{TM}}$ EZ Access ${ }^{\mathrm{TM}}$ Class A volumetric flasks (Fisher Scientific, Fair Lawn, NJ, USA). 
Table 6. Heroin Standards Serial Dilutions

\begin{tabular}{ll}
\hline ppm & Stock solution $(\mu \mathrm{L})$ \\
\hline 200 & 100.0 \\
225 & 112.5 \\
250 & 125.0 \\
275 & 137.5 \\
300 & 150.0 \\
325 & 162.5 \\
350 & 175.0 \\
375 & 187.5 \\
\hline
\end{tabular}

*After stock solution aliquot was added to the volumetric flask, solvent (methylene chloride) was used to complete up to the mark

\subsection{Methods}

\subsubsection{Sampling locations}

Solid phase microextraction fibers are very fragile and will break with the slightest impact therefore, they were transported in their original box inside a 1150 Pelican case. Another important aspect to consider when performing field sampling is to preserve the integrity of the samples in the time between when the sample is collected and the instrumental analysis. Thus, to avoid any source of contamination, SPME fibers were protected using polytetrafluoroethylene (PTFE) sealing caps from Sigma (Sigma-Aldrich, Co., St. Louis, MO, USA). Larroque et al., studied the fiber capacity for preservation of samples after extraction have been performed, and it was concluded that fibers can be stored up to 2 days before use (137). All samples were therefore desorbed soon after they were collected. Since samples were collected locally, the time between the collection and 
desorption of the samples was limited to 25-30 min which satisfies the time frame established for full recovery of the sample. Finally, although environmental conditions were not always similar to controlled room conditions, humidity and temperature was always monitored.

\subsubsection{Extraction Method}

Triplicate samples were weight, transferred, and sealed in brand new $10 \mathrm{~mL}$ screw-top clear vials with polypropylene hole caps and PTFE/silicone septa (Supelco, Bellefonte, PA). The extraction method was optimized as specified in Table 7.

Table 7. Optimized Parameters for SPME Extraction

\begin{tabular}{lll}
\hline Drug & Equilibrium (min) & Extraction (min) \\
\hline Marijuana & 15 & 60 \\
Heroin & 45 & 30 \\
\hline
\end{tabular}

Once samples were prepared for extraction, they were all let sit to reach equilibrium for the already established amount of time (marijuana $15 \mathrm{~min}$, heroin $45 \mathrm{~min}$ ) known to be suitable for a good representation of what the headspace of the drugs contain. To have a set control of the time samples needed to be ready for extraction a Fisher Scientific ${ }^{\mathrm{TM}}$ Traceable $^{\text {TM }}$ Multi-Colored Timer was used (Fisher Scientific, Fair Lawn, NJ, USA). Once equilibrium time was reached, using the adjustable needle gauge the manual holder was set to $1 \mathrm{~cm}$. SPME fiber was then injected in the samples through the septa, hold in place using a stand, and exposed to the headspace of the sample by pushing plunger of the holder and 
locking it in place through the Z-slot securing the fiber in the exposed position during the extraction time.

\subsubsection{GC/MS Method}

The GC used in this experiment was equipped with a temperature programmable inlet (TPI) injector. The GC was fitted with a $30 \mathrm{~m}$ x $0.250 \mathrm{~mm}$ i.d. HP-5MS column coated with a $0.25 \mu \mathrm{m}$ film of stationary phase (Agilent Technologies, Santa Clara, CA, USA). The injector was equipped with a $2 \mathrm{~mm}$ i.d. liner (Supelco, Bellefonte, PA, USA) (in order to obtain better peak shapes) and was operated in splitless/split mode with a splitless time of $1 \mathrm{~min}$.

\section{Marijuana}

The column temperature was held at $40^{\circ} \mathrm{C}$ for 3 min after the injection, then programmed at $10^{\circ} \mathrm{C} / \mathrm{min}$ to $250^{\circ} \mathrm{C}$. Helium was used as a carrier gas at a constant linear velocity of $35 \mathrm{~cm} / \mathrm{s}$. To determine the concentration of the compounds in the sample a set of known concentration standards was analyze to further create a standard calibration curve. To analyze the series of standards across a range of concentrations (5 ppm- 100 ppm) an automatic liquid sampler (ALS) method was created. The GC-MS data acquisition started after the elution (solvent delay $5 \mathrm{~min}$ ) of methylene chloride $\left(\mathrm{MeCl}_{2}\right)$ (used solvent). To avoid any source of contamination and/or carry over, syringe was pre-watched twice before $1 \mu \mathrm{L}$ liquid standard was injected. 


\section{Heroin}

The column temperature was held at $45^{\circ} \mathrm{C}$ for 5 min after the injection, then programmed at $15^{\circ} \mathrm{C} / \mathrm{min}$ to $130^{\circ} \mathrm{C}$ where it was held for $5 \mathrm{~min}$. After that temperature increased $25^{\circ} \mathrm{C} / \mathrm{min}$ to $160^{\circ} \mathrm{C}$, where it was held for 0.5 min and later kept increasing $25^{\circ} \mathrm{C} / \mathrm{min}$ to its final temperature $250^{\circ} \mathrm{C}$. Helium was used as a carrier gas at a constant linear velocity of $34 \mathrm{~cm} / \mathrm{s}$. To determine the concentration of the compounds in the sample a set of known concentration standards was analyze to further create a standard calibration curve. To analyze the series of standards across a range of concentrations (200 ppm- 375 ppm) an automatic liquid sampler (ALS) method was created. The GC-MS data acquisition started after the elution (solvent delay $1.80 \mathrm{~min}$ ) of acetonitrile (ACN) (used solvent). To

avoid any source of contamination and/or carry over, syringe was pre-watched twice before $1 \mu \mathrm{L}$ liquid standard was injected.

\subsection{Results and Discussion}

\subsubsection{Marijuana}

The volatile components of marijuana samples have been studied as potential means for the detection of the illicit substance by detector canines using already optimized extraction method (Table 7). Previous research has suggested great potential of evaluating different marijuana samples for the application in drug monitoring (138). Local detection canines are weekly trained to detect using dry marijuana samples. To have a more control setting to search for the headspace of marijuana, samples varying in their harvested origin, but equal composition, have been evaluated. The idea is to narrow down the pool of compounds with the greatest frequency of occurrence and relative abundance in the 
headspace of dry marijuana samples to be used as potential training aids for detection canines.

An average from triplicate samples $(n=10)$ obtained from different sources in South Florida are presented in Table 8, as depicted, the variation in headspace composition among samples, was not significant. Previous research have evaluated the headspace of marijuana samples varying in countries and in composition including leaf, stem, and seed parts, and in age ranging from fresh to dry and/ or moldy and no "geographic" trend among samples was found $(136,139,140)$. According to Farooqi and Shukla (1999) the use of growth regulators, or plant hormones, can stimulate plant growth and terpene biosynthesis resulting in beneficial changes in terpenes quality and quantity (141). Although there are several growing conditions used to cultivate marijuana, there is still potential to determine key compounds that could be use to develop a training aid.

Table 8. Composition of the Volatiles in Marijuana Samples

\begin{tabular}{ccccccccccc} 
& \multicolumn{1}{c}{ PERCENTAGE } \\
\cline { 2 - 10 } & A & B & C & D & E & F & G & H & I & J \\
\cline { 2 - 10 } MONOTERPENES & 10.5 & 10.5 & 10.5 & 10.5 & 10.5 & 10.5 & 10.5 & 10.5 & 10.5 & 10.5 \\
SESQUITERPENE & 31.6 & 31.6 & 42.1 & 42.1 & 42.1 & 42.1 & 26.3 & 36.8 & 26.3 & 26.3 \\
OTHER & 42.1 & 42.1 & 31.6 & 47.4 & 31.6 & 36.8 & 31.6 & 36.8 & 42.1 & 36.8 \\
\hline
\end{tabular}

To get the greatest possible training aid for detector canines, its important to be aware of how different methods being used to produce marijuana affect the terpene content of the finished product. From the samples evaluated, the majority of the compounds found in marijuana samples were in fact, terpenes, constituting greater than $50 \%$ of the headspace 
of the samples (Figure 18). The majority of the compounds found in marijuana samples were found to be terpenes, constituting greater than $50 \%$ of the headspace of the samples (Figure 18). This fact agrees with other studies previously published (75,130,142-144). An average of $10.5 \%$ of the terpenes found in marijuana where monoterpenes, the class of terpenes containing 10 carbon molecules $\left(\mathrm{C}_{10} \mathrm{H}_{16}\right)$, while $34.7 \%$ of the terpenes were sesquiterpenes the class composed of fifteen carbon molecules $\left(\mathrm{C}_{15} \mathrm{H}_{24}\right)$. In general, terpenes are emitted by many species of vegetation and are best known as the compounds responsible for the pleasant odor of pine forests (145). Although, terpenes are commonly found in plants, a study have successfully shown that marijuana terpenes are distinguishable from other common plants that could conceivably be confused with Cannabis (146). Therefore, is not just the appearance of terpenes that makes marijuana have a unique odor, but rather a combination of them. In fact, the same theory have been suggested for cocaine, after a controversial study demonstrated that landscaping flowers such as snapdragon and petunia flowers, were known to release methyl benzoate, the signature odor of cocaine questioning thus canines capabilities $(147,148)$. However, Cerreta et al., proved the ability of detection canines to distinguish between the odor of cocaine and the odor of snapdragon flowers by conducting canine odor recognition tests (ORTs) (149). Detection canines did not alert to any of snapdragon flowers presented, while all canines evaluated alerted to the positive control (cocaine). 


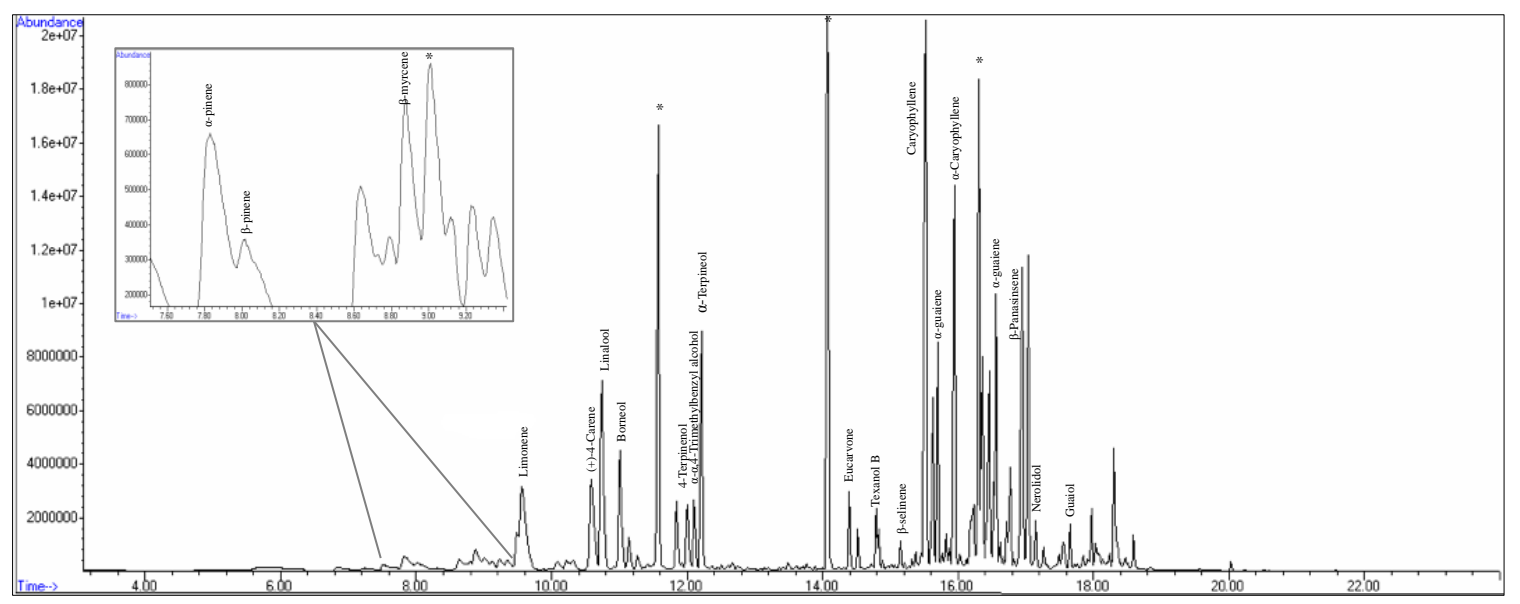

Figure 18. Marijuana HS-SPME Chromatogram (DVB/CAR/PDMS: 15 min Equilibrium, 45 min Extraction)

An average of 32 compounds were identified from the headspace of marijuana, from which only 16 compounds ( $\alpha$-Pinene, (-)- $\beta$-Pinene, $\gamma$-Terpinene, $p, \alpha, \alpha$-Trimethylbenzyl alcohol, $\alpha$-Terpineol, Guaiol, Limonene, 3-Carene, Linalool, (-)-Alloaromadendrene, Benzaldehyde, $\beta$-Myrcene, Fechyl alcohol, Benzothiazole, Caryophyllene, $\alpha$ Caryophyllene) were commonly extracted $75 \%$ or more of the time. The common appearance of these compounds in the headspace samples must not be the only fact to be considered as the goal is to develop a training aid that mimics the odor of the illicit substance. Therefore, the relative abundance of compounds in the headspace is also been studied as another parameter for the potential development of the training aid.

Benzaldehyde, $\beta$-myrcene, fechyl alcohol, benzothiazole, caryophyllene, and $\alpha$ caryophyllene were found in every (100\%) marijuana samples while, limonene, 3-carene, linalool, and (-)-alloaromadendrene were found in most, but not all of the samples (83\%). 


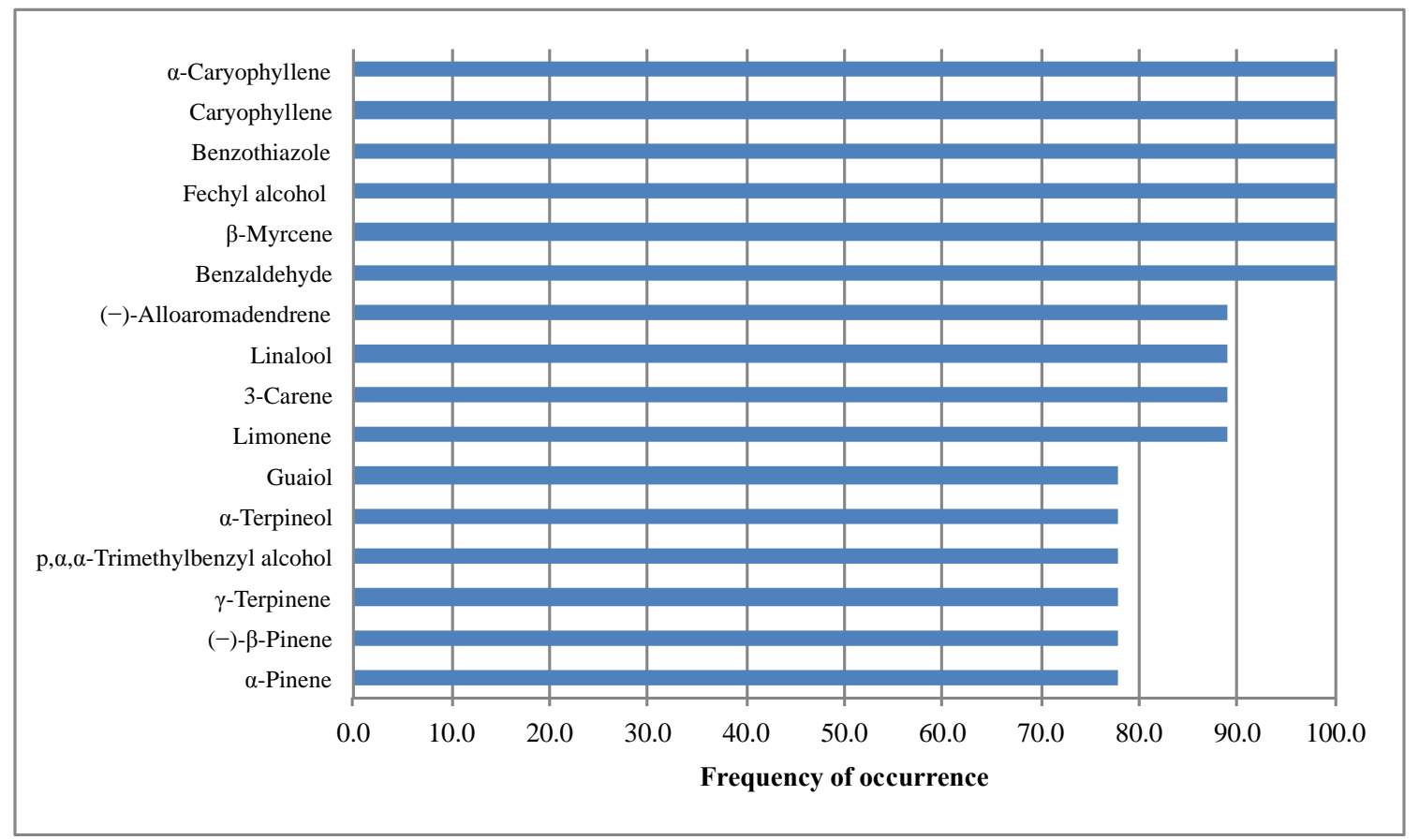

Figure 19. Compounds Frequency of Occurrence (\%) in Marijuana Samples $(n=10)$

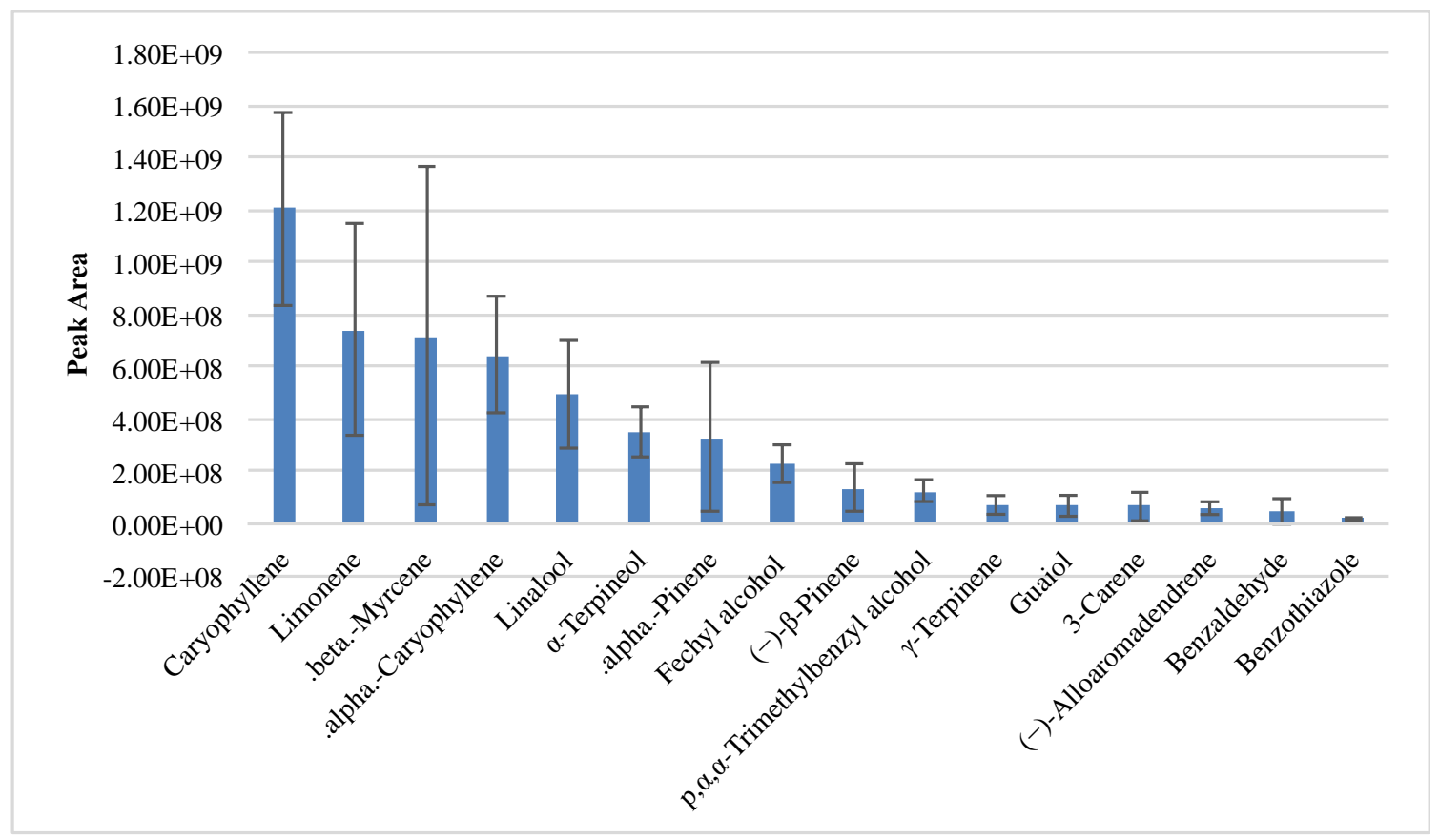

Figure 20. Relative Abundance of Compounds in Marijuana Samples 
When we then consider the abundance of these compounds in the headspace, one notices that caryophyllene and limonene are the compounds that contribute the most to the headspace (Figure 20). The error bars have been included in this figure as an indication of the variability of abundance in the headspace. As depicted, although $\beta$-myrcene and $\alpha$ pinene are compounds widely found in the headspace of the samples, the uncertainty of their quantity exists.

Table 9 shows more in depth information of the composition of terpenes in the headspace of marijuana. For better understanding compounds have been organized in order of average contribution. The relative abundance of caryophyllene is understandable as it is known to contribute larger amounts in dried samples while much less in fresh samples (136). In general, the fact that sesquisterpenes constitute a larger amount of terpenes can be explain on the basis of marijuana composition, as it has been found differences in the headspace depending on whether the sample is fresh, dry or moldy. When marijuana is fresh, monoterpenes predominate in the headspace, but when the sample starts drying over time, sesquiterpenes start increasing as result of thermal rearrangement of certain monoterpenes.

PCA (Figure 21) suggested the existence of two principal clusters of unequal within different sections of a marijuana plant: leaves and dried buds. Although the parts of the plant shared $43 \%$ of the terpenes, when the complete profiles of VOCs are compared, the leaves form a cluster separated from the rest. This highlights the differences between profiles and the reason why canines are strictly trained on dried marijuana. 
Table 9. Contribution (\%) of Compounds in Marijuana Headspace

\begin{tabular}{llllllllllll}
\hline Compounds & $\mathrm{A}$ & $\mathrm{B}$ & $\mathrm{C}$ & $\mathrm{D}$ & $\mathrm{E}$ & $\mathrm{F}$ & $\mathrm{G}$ & $\mathrm{H}$ & $\mathrm{I}$ & $\mathrm{J}$ & Av. \\
\hline Caryophyllene & 30.1 & 23.4 & 23.7 & 16.7 & 29.8 & 20.4 & 29.4 & 26.0 & 22.7 & 35.5 & $\mathbf{2 5 . 7}$ \\
Linalool & 2.5 & 4.3 & 8.0 & 12.9 & 4.0 & 8.7 & 43.5 & 29.6 & 16.7 & 8.9 & $\mathbf{1 3 . 9}$ \\
$\alpha$-Caryophyllene & 15.6 & 14.2 & 12.8 & 9.8 & 15.7 & 11.9 & 13.3 & 12.2 & 11.2 & 20.0 & $\mathbf{1 3 . 7}$ \\
$\beta$-Myrcene & 11.5 & 15.6 & 17.9 & 18.7 & 10.4 & 16.6 & 0.0 & 6.2 & 15.7 & 17.7 & $\mathbf{1 3 . 0}$ \\
Limonene & 12.9 & 14.3 & 13.5 & 8.8 & 13.8 & 12.7 & 0.0 & 6.5 & 6.9 & 6.1 & $\mathbf{9 . 6}$ \\
$\alpha$-Terpineol & 10.6 & 8.4 & 7.4 & 5.0 & 10.0 & 7.5 & 0.0 & 7.3 & 0.0 & 0.0 & $\mathbf{5 . 6}$ \\
$\alpha$-Pinene & 7.7 & 5.6 & 5.7 & 3.5 & 5.9 & 5.0 & 2.1 & 4.9 & 5.4 & 4.2 & $\mathbf{5 . 0}$ \\
Fechyl alcohol & 0.0 & 5.1 & 1.7 & 10.7 & 2.9 & 5.2 & 3.0 & 0.0 & 11.1 & 0.0 & $\mathbf{4 . 0}$ \\
Borneol & 3.2 & 2.7 & 3.3 & 2.2 & 3.7 & 3.0 & 0.0 & 0.0 & 1.8 & 2.0 & $\mathbf{2 . 2}$ \\
p, $\alpha, \alpha$-Trimethylbenzyl alcohol & 0.0 & 0.0 & 2.4 & 4.0 & 0.3 & 2.3 & 3.4 & 1.4 & 3.3 & 0.0 & $\mathbf{1 . 7}$ \\
$\gamma$-Terpinene & 1.7 & 0.4 & 0.0 & 0.7 & 1.8 & 0.9 & 0.0 & 2.4 & 1.9 & 2.2 & $\mathbf{1 . 2}$ \\
Guaiol & 1.2 & 1.6 & 1.7 & 2.6 & 1.0 & 1.7 & 0.8 & 0.4 & 0.0 & 0.4 & $\mathbf{1 . 2}$ \\
(-)- $\beta$-Pinene & 1.0 & 2.2 & 0.0 & 1.2 & 0.0 & 1.1 & 1.3 & 0.8 & 2.1 & 1.2 & $\mathbf{1 . 1}$ \\
3-Carene & 1.0 & 1.0 & 1.0 & 1.0 & 0.0 & 0.9 & 2.2 & 1.1 & 0.5 & 1.5 & $\mathbf{1 . 0}$ \\
(-)-Alloaromadendrene & 0.6 & 0.7 & 0.5 & 2.0 & 0.3 & 1.8 & 0.6 & 0.9 & 0.3 & 0.4 & $\mathbf{0 . 8}$ \\
Benzaldehyde & 0.4 & 0.4 & 0.4 & 0.3 & 0.4 & 0.4 & 0.4 & 0.4 & 0.3 & 0.0 & $\mathbf{0 . 3}$ \\
\hline
\end{tabular}




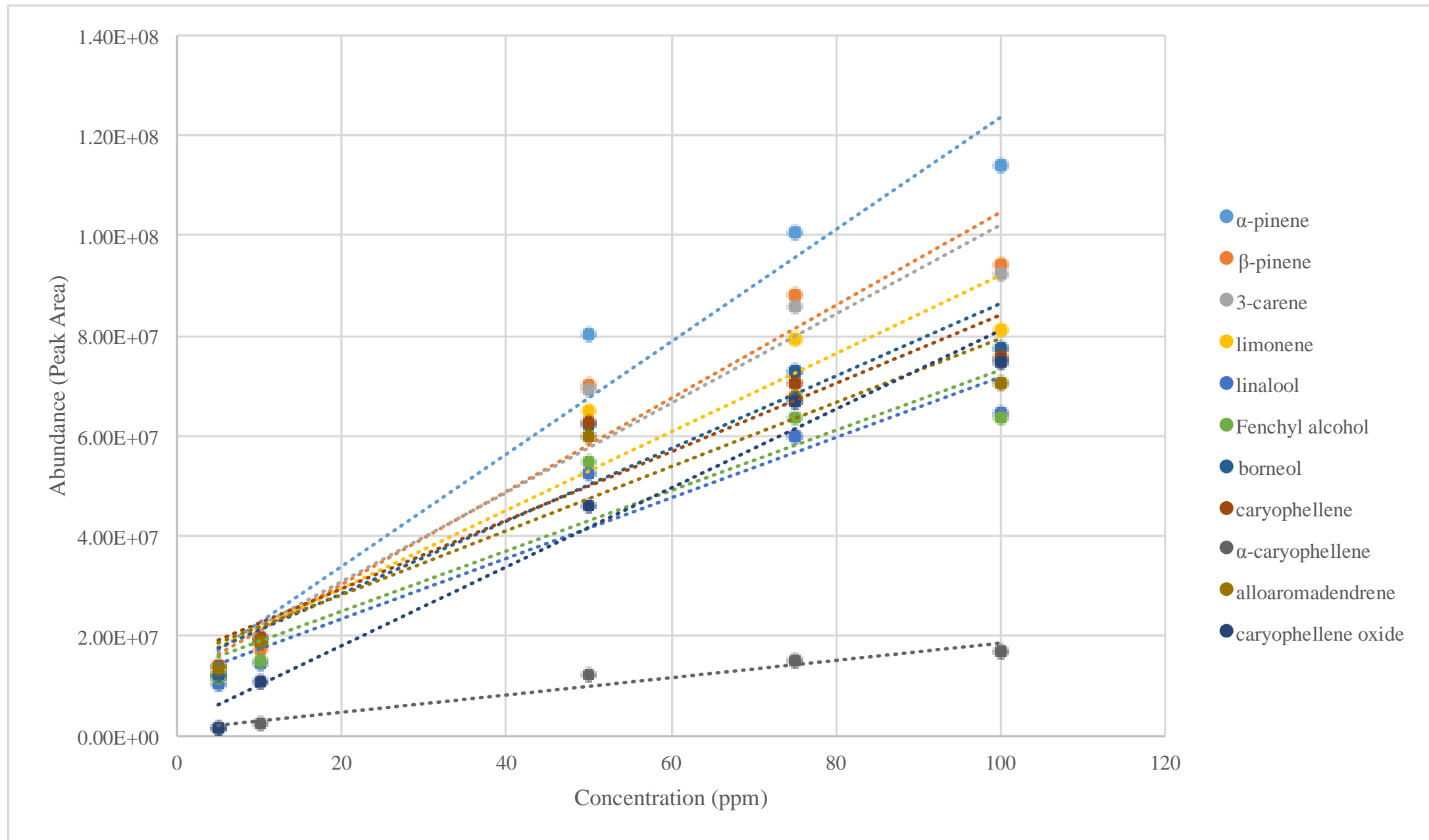

Figure 21. Calibration curves for Marijuana Most Frequent Headspace Compounds 


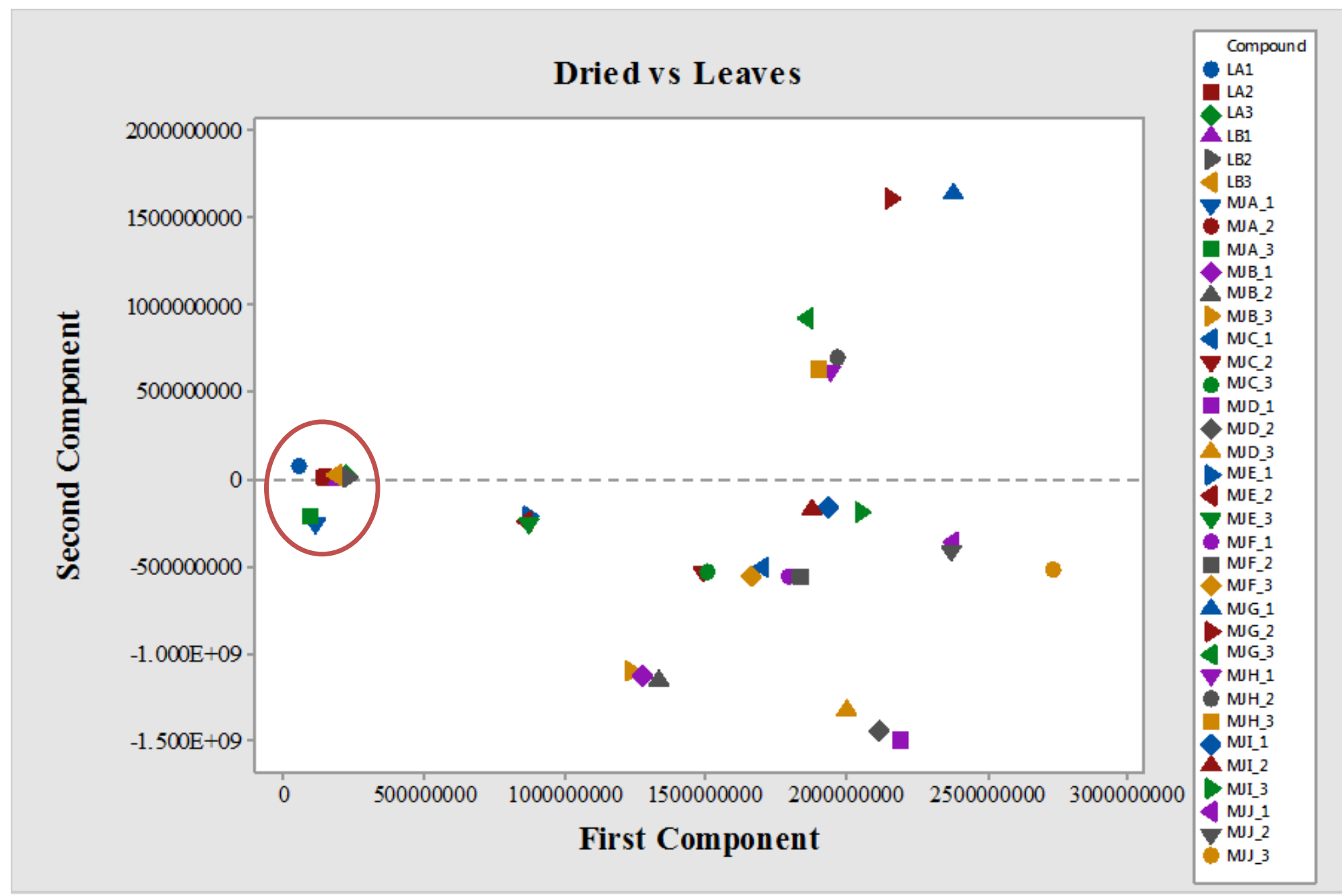

Figure 22. PCA Dried vs Leave Marijuana Samples 


\subsubsection{Heroin}

The importance of characterizing the volatiles associated with heroin comes after the goal to develop an optimal training aid for detector canines. Thus, the composition of volatiles in the headspace of heroin samples has been studied. The figure below presents an example of a chromatogram from one heroin sample from which the VOC profile its been represented. The chromatographic peaks are well-separated, symmetrical, and sharp. The small size $2 \mathrm{~mm}$ i.d. splitless liner in the GC injection port improved the GC resolution compared to the $4 \mathrm{~mm}$ i.d. liner.

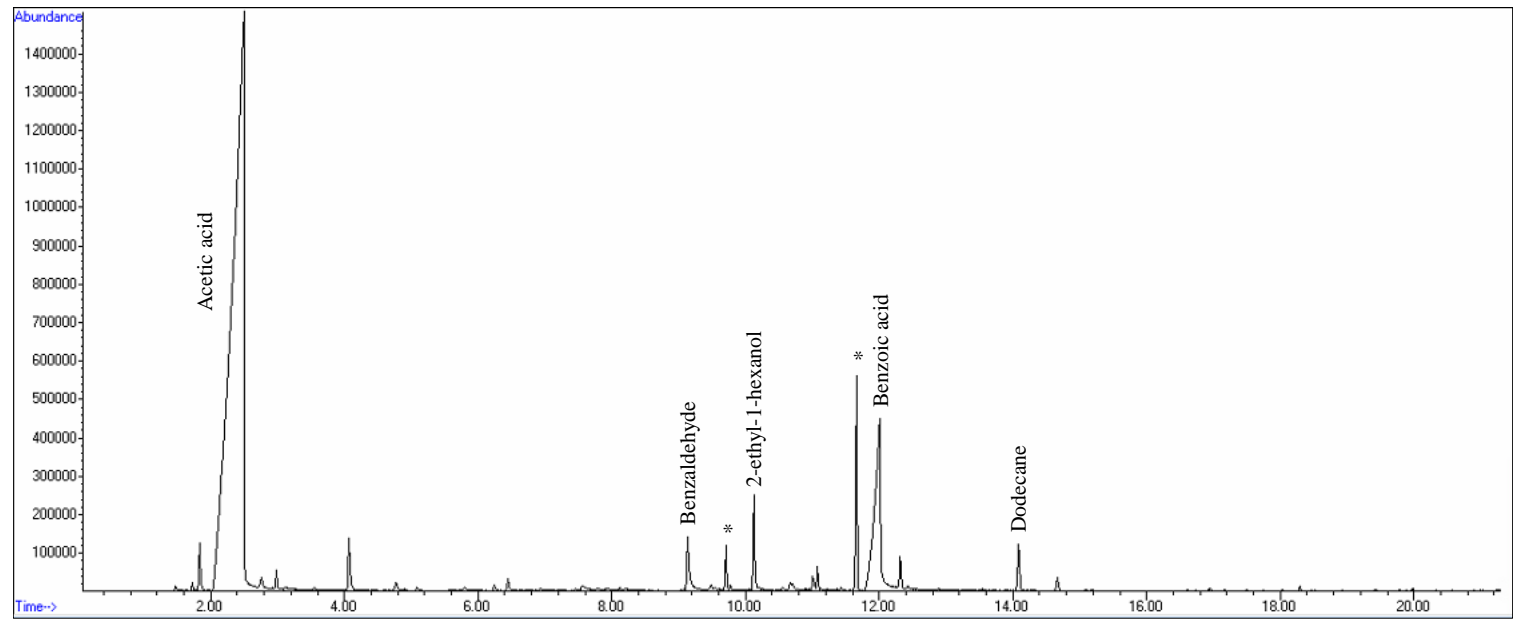

Figure 23. Heroin HS-SPME Chromatogram (DVB/CAR/PDMS: 45 min Equilibrium, 30 min Extraction)

As depicted in the figure below, all the fibers desorbed gave reproducible detection of acetic acid. The presence of acetic acid in the headspace of heroin could be explained by its direct relationship with acetic anhydride. Acetic anhydride is essentially the condensation of two molecules of acetic acid and key precursor chemical used in the illicit manufacture of heroin. Previous research has indicated the reason for the presence of acetic 
acid is due to heroin deacetylation process, which is responsible for its characteristic odor (150). Additionally, we could consider that as an alternative to control, reduce and potentially prevent illicit manufacture and trafficking of narcotic drugs, the precursors used to develop illicit substances, including heroin, have been monitored and reported by International Narcotics Control Board (INCB). As a matter of fact, the number of seizures of acetic anhydride has declined in Afghanistan, possibly due to change of trafficking routes and/or modi operandi used by traffickers. The lack of acetic anhydride has caused the development of alternative synthesis including the use of lower quality precursors such as acetic acid. For instance, Odell et al., published how it is possible to treat morphine with a mixture containing acetic acid and still afford good yields of heroin (34).

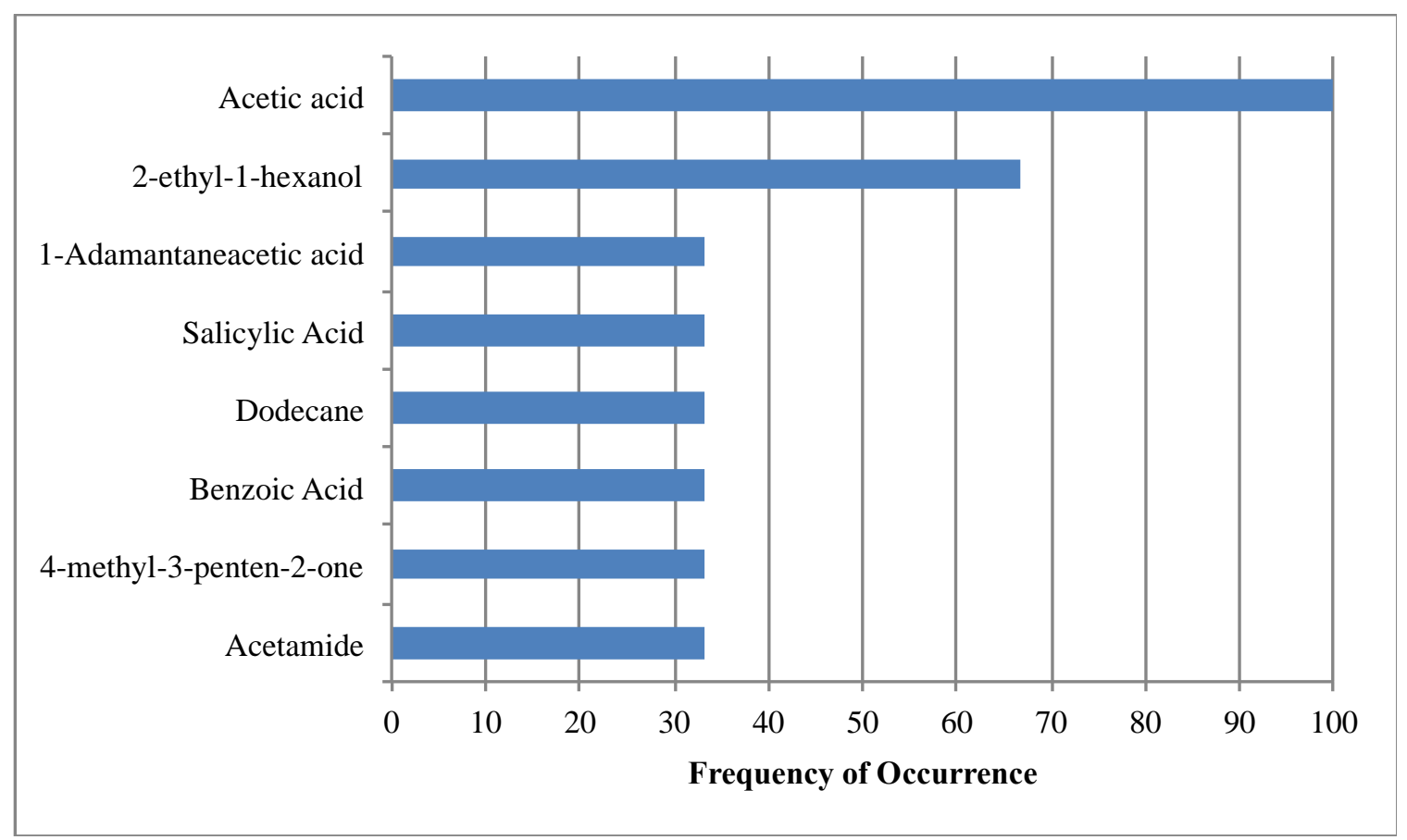

Figure 24. Compounds Frequency of Occurrence (\%) in Heroin Samples (n=5) 
Not only acetic acid is the most frequently occurring compound in the headspace of the samples, but also the most abundant. In fact, acetic acid constitutes $85 \%$ of the abundance of compounds in the headspace. When compared with other types of heroin, such as "black tar"; a lower pure form of heroin (151), the most abundant volatiles is still acetic acid.

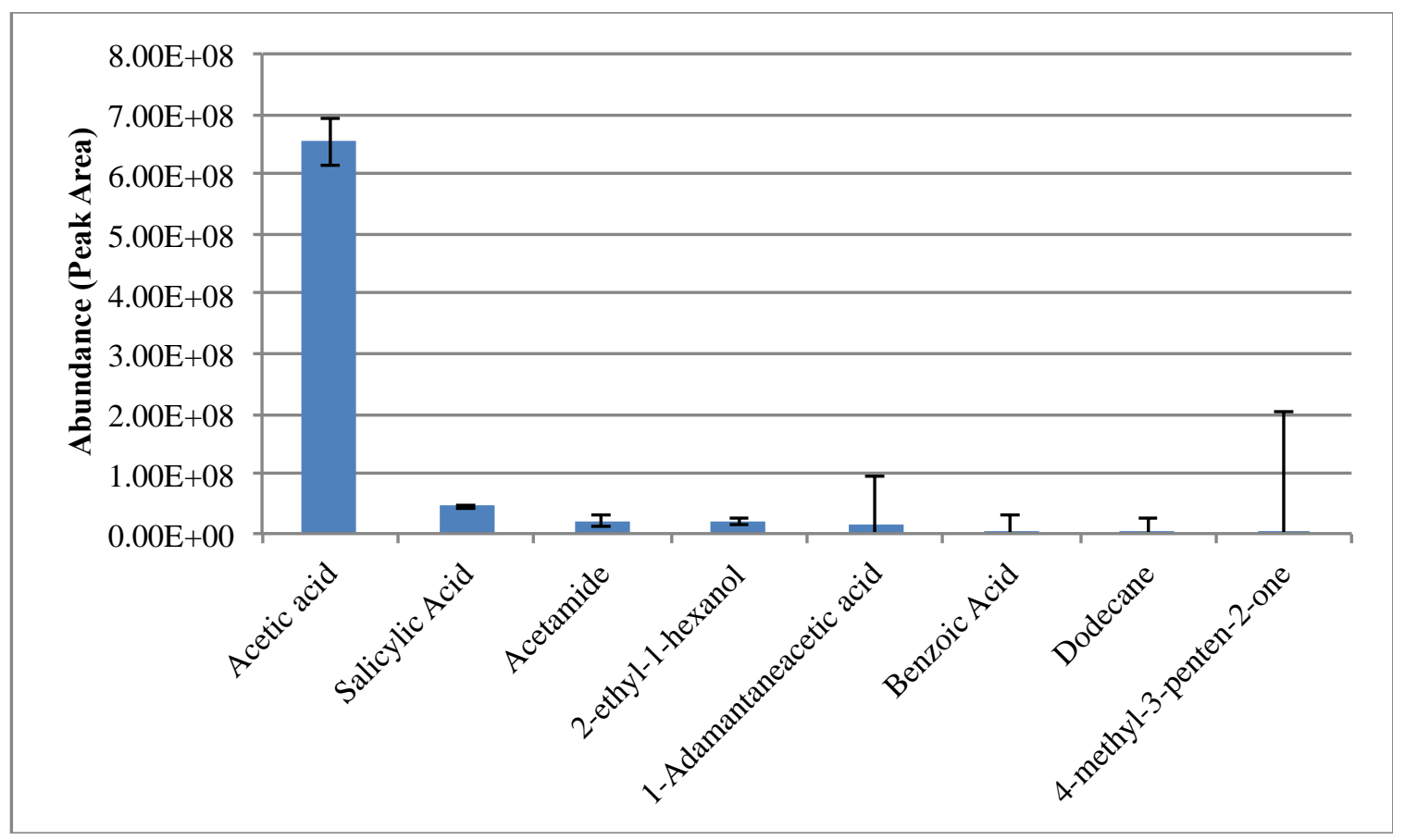

Figure 25. Relative Abundance of Compounds in the Headspace of Heroin Samples 


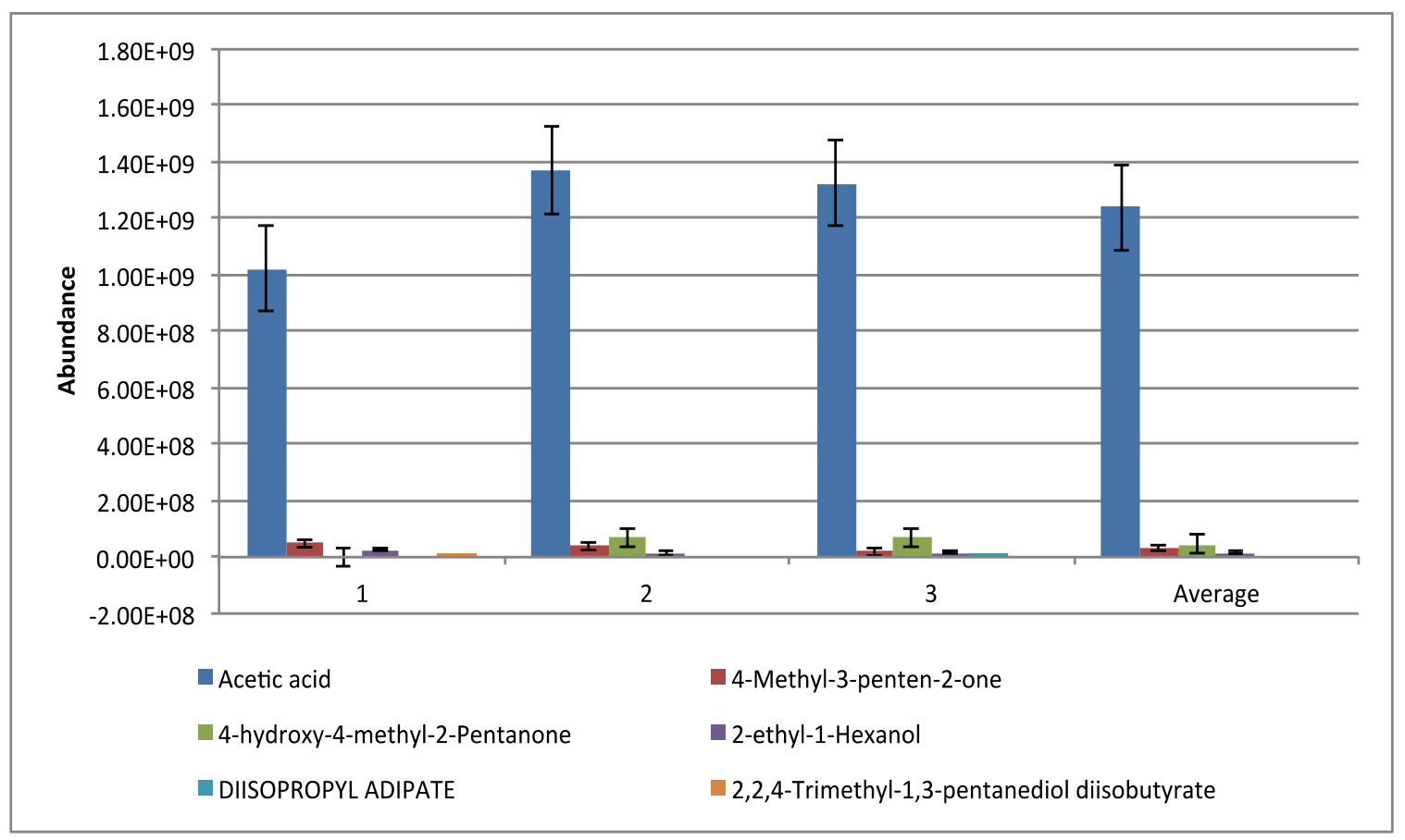

Figure 26. Triplicate of the Headspace of "Black Tar" Heroin Samples

Previous research have evaluated the use of acetic acid as a training aid for detection canines as it has been conjectured that acetic acid is the dominant odor compound in heroin samples, unfortunately, no success was achieved. Only one canine alerted (102) to a gauze spiked with $100 \mu \mathrm{L}$ of acetic acid (9), These results highlights the need of ORTs considering mixture of compounds rather than the only acetic acid.

\subsection{Conclusion}

The headspace of marijuana and heroin samples provided by authorized personnel in South Florida was evaluated to determine VOCs constituting the profile of these substances. Extraction parameters optimized in the previous task were utilized to analyze the samples. Triplicates of fiber (DVB/CAR/PDMS) were injected to the headspace above the sample and to a blank after vials were let sit to reach equilibrium. After extraction was 
completed, one fiber was desorbed through the inlet of the GC-MS while the rest were caped to be preserved. The results show that it is possible to evaluate the volatiles even when time and sample quantity is a limitation, as SPME it is a simple, fast and sensitive technique. Also, it is feasible to obtain promising results without having to alter the sample, as it is a non-destructive, not invasive extraction technique.

As expected, marijuana headspace is composed of terpenes; organic hydrocarbons produced by a wide variety of plants and that are known to be responsible of the scent that is expelled by the product. From the terpenes that were obtained, sesquiterpenes predominated the headspace. Studies have shown that depending on the physical characteristics of the samples evaluated is the type of terpenes that are prone to obtain. In fact, all samples evaluated were dried; sesquiterpenes predominated in the profile. From all the compounds, caryophyllene, $\beta$-myrcene, linalool and limonene dominated not only in frequency of occurrence but also in abundance in the headspace giving of a pointer of what could be use as potential training aids for detection canines.

Additionally, the headspace of heroin samples has been evaluated to determine those compounds that are most frequently found as well as those that are more abundant. Acetic acid greatly predominated in the headspace, although acetic acid was not widely used initially when heroin was first developed, its presence can be explained by the deacetylation of acetic anhydride, common precursor compound used for the synthesis of heroin. Also, the fact that such precursor compounds have been monitored for its trafficking have impulse the use of acetic acid instead. Although its use requires a much more complex synthesis, studies have shown its potential to get high yields of heroin. Finally, regardless of the type of heroin studied, acetic acid prevailed as the most abundant 
compound in heroin, which highlights its importance in heroin, but also the need for further ORTs approaches to address its potential means for the development of detector canine training aids.

These studies should allow the development of training aids which are safer to use, easy to acquire and provide consistent levels of chemicals. Compounds found in the headspace of both illicit substances will be used to potentially determine the compound and/or mixture of compounds responsible for the alert response of canines which is essential to improve their performance and reliability.

\section{TASK 3. ACTIVE ODOR DETERMINATION USING BIOLOGICAL DETECTORS}

\subsection{Introduction}

Different specialized search techniques are often use to locate an object of interest. Depending upon the issues to be addressed investigators may do search themselves or use technical methods such as instrumental detectors and/or trained detector dogs. However, law enforcement has relied on canines to detect narcotics during traffic stops, vehicle checkpoints, ports-of-entry, private homes and businesses, and while searching the luggage of travelers aboard buses, trains, and airplanes (152). Over the years, canines have continuously prove their accuracy and sensitivity to detect illicit substances even in the presence of distractors. Canines have also demonstrated to be fast to work large surface areas, long working cycles, and in any environmental condition (135). Yet, their efficiency greatly depends on the frequency of training hours which is been limited by the facility of which training aids can be obtained. To get access to these substances, local, state, and federal laws require licensing which often mandates background investigation, interviews 
and documentation to acknowledge specific facility requirement to access the stored substances. For instance, the storage must contain regulations that include but are not limited to restricted access, monitored security, biohazard protection, fire protection, theft detection, among others (153). Now, once materials are accessible, there were still several problems such as acquisition cost, transport considerations, permits, knowledge, equipment and training for each person involved. Additionally, the typical training aid supplied is not necessarily pure, as they are generally street drugs some of which have not been previously tested. Sometimes, these substances have not even been stored properly increasing thus the possibility of contamination. The contamination not only could be followed by poor storage, but can also be product of the cutting agents that are often added to dilute the drug but increase the quantity of the product. To provide law enforcement with an alternative to train on a daily basis, sufficing the parameters required to be proficient, marijuana and heroin training aids will be designed as an equivalent of current venues for detection training. These training aids are meant to reinforce current practices by allowing canine teams to easily obtain a product that will provide equivalent characteristics to the illicit substances but without the drawbacks associated with the use of real materials.

\subsection{Materials}

A combination of the most frequently found and most abundant compounds in the headspace of marijuana and heroin: $\alpha$-pinene, $\beta$-pinene, limonene, linalool, and $\beta$ caryophellene, benzoic acid, salicylic acid and acetyl salicylic acid were obtained from Sigma (Sigma-Aldrich, Co., St. Louis, MO, USA). Dukal ® 2x2 sterile gauze pads (Dukal 
Co., Ronkonkoma, NY, USA) were spiked and place in $10 \mathrm{~mL}$ screw-top clear vials with polypropylene hole caps and PTFE/silicone septa (Supelco, Bellefonte, PA,USA).

Trained and certified law enforcement narcotic detector canine teams willing to participate in the study were scheduled for field trials depending on their availability. Name, breed, age and gender were recorded to keep record of participants.

Table 10. Certified Canines: Breed, Age and Gender Data

\begin{tabular}{l|llll}
\hline CANINE NO. & NAME & BREED & AGE & F/M \\
\hline $\mathbf{1 2}$ & Nina & Labrador & 9 & F \\
$\mathbf{1 3}$ & Thor & Shepherd & 5 & M \\
$\mathbf{1 6}$ & Ricco & Malinois & 8 & M \\
$\mathbf{2 1}$ & Sting & Malinois & 6 & M \\
$\mathbf{2 2}$ & Jake & Malinois & 2.5 & M \\
$\mathbf{2 3}$ & Milo & Shepherd & 5 & M \\
$\mathbf{2 4}$ & Apache & Malinois & 7 & M \\
$\mathbf{2 7}$ & Loki & Shepherd & 6 & M \\
$\mathbf{2 8}$ & Doc & Shepherd & 5 & M \\
$\mathbf{2 9}$ & Kobee & Malinois & 2 & M \\
$\mathbf{3 0}$ & Shadow & Shepherd & 2 & M \\
$\mathbf{3 1}$ & Tango & Labrador & 5 & M \\
\hline
\end{tabular}

\subsection{Methods}

To resemble normal operational conditions, trials were performed in warehouses and/or buildings. The Scientific Working Group on Dog and Orthogonal detector Guidelines (94) was followed as it establishes the best practices for the use and evaluation 
of detector canines. SC8 Substance Dogs: Narcotics establishes that when evaluations in Building/room is been performed the room may contain zero to three aids depending upon the size and environmental conditions, and should be $18.6-111.5 \mathrm{~m}^{2}$ (200-1200 sq. ft.) with furniture. Specifics on the buildings/room where the trials were performed can be found in the Appendix section. Training aids (hides) were placed no less than 30 minutes prior to testing. In order to demonstrate the ability to detect the trained odors, positive control (marijuana) and a blank were always included. Although the handlers were advised of the parameter of search, teams did not know the placement of the hides. Handlers were only allowed to reward the canine when they positive alerted to the positive control otherwise alerts were only recorded.

\section{Marijuana Single Compound Training Aid}

Five training aids were prepared by placing double gauze pads into $10 \mathrm{~mL}$ vials using tweezers and spiking $200 \mu \mathrm{L}$ of each standard compound into separate Dukal ${ }^{\circledR}$ gauzes using Eppendorf® Research ${ }^{\circledR}$ plus pipette. For preservation and transportation purposes, vials were sealed using Parafilm Sealing Film (Sigma-Aldrich, Co., St. Louis, MO, USA) which was later removed for the test.

\section{Marijuana Multi-compounds Training Aid}

Five training aids were prepared by placing double gauze pads into $10 \mathrm{~mL}$ vials using tweezers and spiking $200 \mu \mathrm{L}$ of each standard compound into separate Dukal® gauzes using Eppendorf® Research® plus pipette. For preservation and transportation purposes, vials were sealed using Parafilm Sealing Film (Sigma-Aldrich, Co., St. Louis, MO, USA) which was later removed for the test. 


\section{Heroin Multi-compounds Training Aid}

Three training aids were prepared by adding 3:1 ratio of each standard compound into. $10 \mathrm{~mL}$ vials using. For preservation and transportation purposes, vials were labeled and sealed using Parafilm Sealing Film (Sigma-Aldrich, Co., St. Louis, MO, USA) which was later removed for the test.

\subsection{Results and Discussion}

\subsubsection{Marijuana}

It has been previously suggested that terpenes may be responsible for the canine alert of marijuana. Although terpenes are high volatile compounds, and are also the main chemical compounds found in the headspace of marijuana samples, is the combination of these compounds in the headspace, what makeup marijuana's profile unique. Previous research have shown that when canines are presented with a combination of five compounds: $\alpha$-pinene, $\beta$-pinene, myrcene, limonene, and $\beta$-caryophyllene poor alert response is obtained (9). Therefore, fresh single-compound training aids, chosen based on the frequency and abundance in the headspace, were first evaluated. To prepare the training aids, individual gauze pads were placed in $10 \mathrm{~mL}$ vials and were individually spiked with $200 \mu \mathrm{L}$ of each compound (limonene, $\alpha$-pinene, caryophyllene, $\beta$-pinene, and linalool). Additionally, to proof the teams were in good working conditions, a positive control (marijuana) was included as part of the test.

Triplicates of eight canines were evaluated in different scenarios, on different days. The handlers were instructed to have their canines sample the odor in each scenario and identify a response of alert, no-alert, or interest. The change of behavior towards a specific 
location without giving a final response was considered for the study as interest, but was only recorded following the handler's indication. The total number of trials per canines is represented in the denominator of the fraction while the number of alerts was recorded in the numerator. All canines alerted to the marijuana samples regardless of the location and/or environmental conditions, indicating the proficiency of their work.

Table 11. Individual Canine Performance Table on Potential Pseudo Marijuana Training Aid (Numerator=A, Denominator $=$ No alert, Parenthesis $=$ Interest)

\begin{tabular}{ccccccc}
\hline Canine & Limonene & $\boldsymbol{\alpha}$-Pinene & Caryophyllene & $\boldsymbol{\beta}$-Pinene & Linalool & PC \\
\hline $\mathbf{1 2}$ & $1 / 3(1)$ & $2 / 3$ & $0 / 3$ & $2 / 3(1)$ & $1 / 3(1)$ & $3 / 3$ \\
$\mathbf{1 6}$ & $2 / 3$ & $3 / 3$ & $3 / 3$ & $1 / 1$ & $3 / 3$ & $3 / 3$ \\
$\mathbf{2 1}$ & $2 / 3(1)$ & $2 / 3(1)$ & $1 / 3$ & $2 / 3$ & $1 / 3$ & $3 / 3$ \\
$\mathbf{2 2}$ & $2 / 3(1)$ & $1 / 3$ & $2 / 3(1)$ & $1 / 2$ & $1 / 3$ & $3 / 3$ \\
$\mathbf{2 3}$ & $0 / 2(2)$ & $1 / 2(1)$ & $0 / 2(1)$ & $1 / 1$ & $1 / 2$ & $2 / 2$ \\
$\mathbf{2 4}$ & $2 / 3(1)$ & $1 / 3$ & $0 / 3(2)$ & $1 / 3$ & $1 / 3$ & $3 / 3$ \\
$\mathbf{3 0}$ & $0 / 3(2)$ & $0 / 3(1)$ & $1 / 3$ & $0 / 1$ & $0 / 3(2)$ & $3 / 3$ \\
$\mathbf{3 1}$ & $1 / 3(2)$ & $0 / 3$ & $1 / 3(1)$ & $0 / 1$ & $2 / 3$ & $3 / 3$ \\
\hline
\end{tabular}

A combine alert rate considers the alert and interest of all canines evaluated. Canines search for compounds frequently found in marijuana lead to a combined alert rate of $50.0 \%$ or more for all compounds except $\alpha$-pinene. For limonene, canines combined alert rate was $100 \%$, while for $\beta$-pinene, caryophyllene, and linalool canines combined alert rate was $60.0 \%, 57.1 \%$, and $50.0 \%$ respectively. Although this data shows promising results, further evaluation has to be done as with a combination of compounds a more unique makeup of marijuana samples could be mirrored. 
Table 12. Odor Recognition Test (Average): Single-Compound Pseudo Marijuana $(n=27)$

\begin{tabular}{ccccc}
\hline Training Aid & Alert Rate $(\%)$ & Interest Rate $(\%)$ & $\begin{array}{c}\text { No Alert } \\
\text { Rate }(\%)\end{array}$ & $\begin{array}{c}\text { Combined Rate } \\
\text { of Detection }(\%)\end{array}$ \\
\hline Limonene & 57.1 & 42.9 & 0.0 & 100.0 \\
Pos. Control & 100.0 & 0.0 & 0.0 & 100.0 \\
$\boldsymbol{\beta}$-Pinene & 60.0 & 0.0 & 40.0 & 60.0 \\
Caryophyllene & 28.6 & 28.6 & 42.9 & 57.1 \\
Linalool & 16.7 & 33.3 & 50.0 & 50.0 \\
a-Pinene & 42.9 & 0.0 & 57.1 & 42.9 \\
\hline
\end{tabular}

Currently, it is not known whether is one compound, or a mixture of compounds what establishes the canine effective match that stimulate a final response. Therefore, volatiles with the greatest combined alert rate were selected to be further evaluated as multicompound mixtures training aid. Gauze pads spiked with $200 \mu \mathrm{L}(1: 1$ ratio) mixture with limonene such as limonene- $\alpha$-pinene, limonene-caryophyllene, and limonenecaryophyllene- $\alpha$-pinene were presented to canines in order to test their response towards the odor been presented. The canines listed in Table 13 sample the odor in each scenario and a response of alert, no-alert, or interest was recorded. As depicted in the table the mixture with the best average response was $\mathrm{C}$ with a combined alert rate of $75 \%$. 


Table 13. Individual Canine Performance Table on Potential Multi-compound
Pseudo Marijuana
\begin{tabular}{cccccc} 
(Numerator=A, & Denominator= No alert, Parenthesis= & Interest) \\
Canines No. & A & B & C & PC \\
\hline $\mathbf{1 2}$ & $0 / 3$ & $1 / 3$ & $3 / 3$ & $3 / 3$ \\
$\mathbf{3 4}$ & $0 / 2$ & $0 / 2$ & $0 / 2(1)$ & $2 / 2$ \\
$\mathbf{3 1}$ & $0 / 4$ & $1 / 4(2)$ & $2 / 4(1)$ & $4 / 4$ \\
$\mathbf{2 9}$ & $0 / 4$ & $0 / 4$ & $0 / 3(2)$ & $4 / 4$ \\
$\mathbf{2 2}$ & $0 / 3$ & $0 / 3$ & $1 / 3(1)$ & $3 / 3$ \\
$\mathbf{1 3}$ & $0 / 1$ & $0 / 1$ & $1 / 1$ & $1 / 1$ \\
\hline
\end{tabular}

Mixtures of VOCs in controlled relative ratios to simulate the odor profile composition of marijuana have not been evaluated before to test their effectiveness in producing suitable canine training aids. Therefore, an average ratio for limonene and caryophyllene in the headspace of marijuana was calculated and considered for a field trial. After evaluating the profile of ten marijuana samples, based on the compounds abundance in the headspace, a relative ratio of 2:3 limonene:caryophyllene was obtained and considered to develop additional variety of the mimic. Gauze pads were spiked with the controlled ratio mixture to further test the response of the canines after been presented with it. Unfortunately, no canines except for one (No.30) showed interest to the 2:3 ratio mixture.

\subsubsection{Heroin}

Various studies have identified the VOCs released from heroin samples. It has been previously suggested that the training compounds need to be representative of the actual sample of interest (i.e. heroin) and distinguishable from other products, such as comestibles (i.e. vinegar); thus, the use of acetic acid as a single training compound may not be sufficient (154). However, the mixture of compounds in controlled ratios to mimic the odor 
profile of heroin have not been considered to develop a suitable training aid. Therefore, the ORT for this particular task involved the mixture of acetic acid with other compounds that were commonly found in heroin samples. Additionally, although it was not shown in the headspace of heroin through SPME, the presence of salicylic acid confirmed the fact that heroin is commonly known to be cut with additional compounds such as acetyl salicylic to stretch the quantity of the drug sold. Therefore, acetyl salicylic acid was also included as one of the mixtures to be evaluated.

Triplicates of ten canines were evaluated in different locations, on different days. The handlers were instructed to have their canines sample the odor in each scenario and identify a response of alert, no-alert, or interest. The change of behavior towards a specific location without giving a final response was considered for the study as interest, but was only recorded following the handler's indication. The total number of trials per canines is represented in the denominator of the fraction while the number of alerts was recorded in the numerator. All canines alerted to the heroin samples regardless of the location and/or environmental conditions, indicating the proficiency of their work.

All canines successfully alerted to the positive control (heroin) while most of them alerted or showed interest to the 3:1 mixture of acetic acid and acetyl salicylic acid. Although acetyl salicylic acid is difficult to analyze by mass chromatography it has been quietly seen in heroin samples as adulterant. In fact, based on 375 heroin samples investigated, the United Nations Bulletin (2007) revealed that street samples often contain various adulterants, among them aspirin (155). Also, in Florida v. Jardines it was stated that "Instead of smelling heroin, drug-detection dogs alert to acetic acid- an odor shared by vinegar and aspirin that is past its prime" (147). 
Table 14. Individual canine performance table on potential pseudo heroin training aid (Numerator $=A$, Denominator $=$ No alert, Parenthesis $=$ Interest $)$

\begin{tabular}{c|ccccc}
\hline CANINE NO. & BZA & SA & BLANK & ASA & PC \\
\hline $\mathbf{1 2}$ & $2 / 3$ & $3 / 3$ & $0 / 3$ & $3 / 3$ & $3 / 3$ \\
$\mathbf{1 3}$ & $1 / 3(1)$ & $3 / 3$ & $0 / 3$ & $3 / 3$ & $3 / 3$ \\
$\mathbf{1 6}$ & $2 / 3$ & $2 / 3$ & $0 / 3$ & $3 / 3$ & $3 / 3$ \\
$\mathbf{2 1}$ & $2 / 3(1)$ & $2 / 3$ & $0 / 3$ & $3 / 3$ & $3 / 3$ \\
$\mathbf{2 2}$ & $2 / 3$ & $3 / 3$ & $1 / 3$ & $2 / 3$ & $3 / 3$ \\
$\mathbf{2 4}$ & $1 / 3$ & $1 / 3(1)$ & $0 / 3$ & $1 / 3(2)$ & $3 / 3$ \\
$\mathbf{2 7}$ & $0 / 3$ & $0 / 3(1)$ & $0 / 3$ & $0 / 3(3)$ & $3 / 3$ \\
$\mathbf{2 9}$ & $1 / 3$ & $1 / 3(2)$ & $0 / 3$ & $1 / 3(1)$ & $3 / 3$ \\
$\mathbf{3 0}$ & $0 / 3(2)$ & $0 / 3(2)$ & $0 / 3$ & $0 / 3(2)$ & $3 / 3$ \\
$\mathbf{3 1}$ & $2 / 3$ & $1 / 3$ & $0 / 3$ & $2 / 3$ & $3 / 3$ \\
\hline PC = Positive Control, ASA = Acetyl salicylic acid, SA = Salicylic acid, BZA = benzoic acid
\end{tabular}

When an average of responses is consider (Table 15) results suggest great potential for the mixture of 3:1 mixture of acetic acid and acetyl salicylic acid. Out of 32 canines evaluated, $90 \%$ of them either alerted or showed interest to the mixture.

Table 15. Odor Recognition Test (Average): Pseudo Heroin $(n=32)$

\begin{tabular}{ccccc}
\hline Training Aids & Alert Rate $(\%)$ & Interest Rate $(\%)$ & $\begin{array}{c}\text { No Alert } \\
\text { Rate }(\%)\end{array}$ & $\begin{array}{c}\text { Combined Rate of } \\
\text { Detection }(\%)\end{array}$ \\
\hline PC & 100 & 0 & 0 & 100 \\
ASA & 60 & 30 & 0 & 90 \\
SA & 50 & 20 & 20 & 70 \\
BZA & 50 & 10 & 30 & 60 \\
Blank & 0 & 0 & 80 & 0 \\
\hline PC = Positive Control, ASA = Acetyl salicylic acid, SA = Salicylic acid, BZA = benzoic acid
\end{tabular}




\subsection{Conclusion}

Odor recognition tests were used to determine if there was any compound(s) that stimulate an alert response when presented to the canines. Additionally, ORTs have been included to evaluate whether or not the canines evaluated are in adequate condition for the test, as odor that they have been trained and certified on, have been included as positive controls (132). A satisfactory work condition was considered when canines successfully alerted to the positive control.

The use of VOC(s) as training aids has been suggested as canines are known to alert to a chemical associated with the drugs rather than the parent drug (8). However, nothing has been established in regards to the number of compounds or the ratio at which compounds could be use to mimic the scent of an illicit substance. Single compounds and/or mixture of compounds suspected to have great potential to be used as training aids for detection canines were evaluated following SWGDOG SC8 Substance Dogs: Narcotics (106). Negative control (blanks) and positive control (drugs) were made available separately. The ORTs performed throughout this study to determine the active odor in marijuana revealed that there are compounds capable to elicit an alert response from detector canines. These compound were not only found to be frequently present in the headspace of the samples, but were also the most abundant. Table 12 summarizes indoor test for eight canines tested more than once $(n=27)$, on different days and in different locations. Single compound training aids were evaluated from which limonene was the most dominant, making $100 \%$ of the canines alert or show interest to it. However, to have a training aid that represents the headspace of the marijuana samples more accurately, different mixtures involving the use of limonene where also evaluated. As it was previously 
stated the best average response was obtained for mixture $\mathrm{C}$ (1:1 limonene: caryophyllene) with a combined alert rate of $75 \%$.

The same protocol was followed for the evaluation of volatiles potentially responsible for the alert response of heroin by detection canines. Single-compound training aids have already been evaluated therefore, the ORTs for this particular drug was to focus on the evaluation of mixture of compounds. As depicted in Table 15 the mixture of compounds with the best response was for 3:1 acetic acid mixture with acetyl salicylic acid with an average response of $90 \%$. Acetic acid was frequently found to be the most abundant compound in heroin headspace while acetic salicylic acid is known to be commonly used as cutting agent.

\section{TASK 4. FORMATION OF CONTROLLED ODOR MIMIC PERMEATION SYSTEMS}

\subsection{Introduction}

Characteristics of ideal training aids include: inertness for safe handling, poses no health risk to the canine; generation of detectable levels of key odorant(s) with high effectiveness relative to real illicit substance and possesses suitable longevity $(134,156,157)$. By using a combination of a good absorbent material inside a polymer bag, a suitable training aid can be develop. The idea is to include an absorbent material that will retain the liquid chemical while the polymer bag will provide a field deployable system with reproducible source of known amounts of target odors (112), and less exposure to contamination. Low-density polyethylene (LDPE) have been previously shown to allow gradual diffusion of the odor (114) and will therefore be used as the permeable bag containing the compounds identified as signature odor. However, nothing is known about 
the capability of absorbents such as alumina and/or cellulose to retain volatiles that could potentially be used as training aids. Alumina is known for its high porous structure and high surface area-to-mass ratio, as well as great properties like high selectivity, high rate of absorbent, high resistant to thermal stock, etc. Another alternative for alumina is cellulose, also an absorbent material but comprise instead of polysaccharides. Besides being an absorbent material, it is also important to consider temperature and humidity, as they are known to affect the volatility of a compound. Therefore; these two parameters will be controlled and recorded on a daily basis. The purpose of this study is then to evaluate different absorbent materials and to determine how these affect the preservation of target explosive odor samples over time while temperature and humidity is been recorded.

\subsection{Materials and Method}

To determine the most suitable training aid construction for detection canines, two different absorbent materials were evaluated, alumina adsorption 80-100 mesh (Fisher Scientific, Fair Lawn, NJ, USA) and cellulose microcrystalline powder $20 \mu \mathrm{m}$ (SigmaAldrich, Co., St. Louis, MO, USA). Thirty grams of each adsorption material were spiked with a mixture of the limonene and caryophyllene (Sigma-Aldrich, Co., St. Louis, MO, USA) for marijuana mimic, while $145.5 \mu \mathrm{L}$ of acetic acid (Fisher Scientific, Fair Lawn, NJ, USA) and $288 \mathrm{mg}$ of acetyl salicylic acid (Sigma-Aldrich, Co., St. Louis, MO, USA) was added to the adsorption materials for heroin mimic. These amounts were selected based on the ratio found in the headspace of each sample. Upon completion, the mixtures were shaken in their corresponding mixing jars utilizing United Nuclear Ball Mill (United Nuclear Scientific, East Lansing, MI, USA) for 5 min every 30 min for 3 hr to achieve an 
homogeneous mixture of odorant. Once the mixture was ready, it was divided into $32 \mathrm{mil}$ LDPE bags containing $10 \mathrm{~g}$ each, these bags were later triple heat sealed three times using an Midwest Pacific impulse heat sealer (Packco Inc., Rocky Mount, MO, USA). The bags were later hanged of a string in a glass tank (20" x 10" x 12") as depicted in the figure below. Each day, samples were removed from the glass tank and placed in a mason jar for daily headspace analysis and/or weighing.

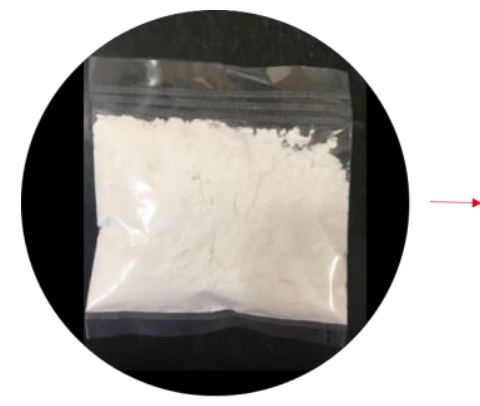

Training aid

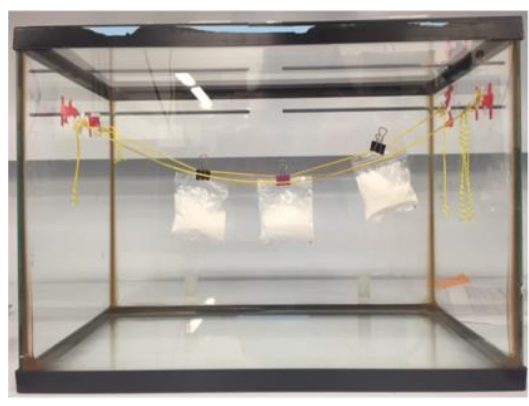

Dissipation setup

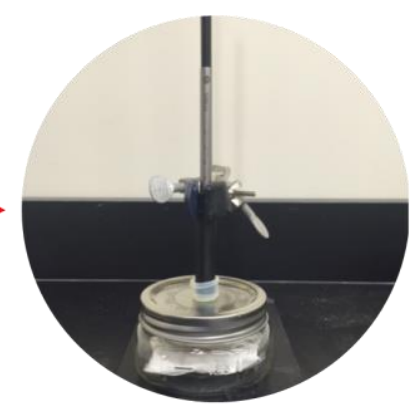

HS-SPME analysis

Figure 27. Methodology for Dissipation Rate Study

\subsection{Results and Discussion}

\subsubsection{Marijuana}

The effective longevity of odor was determined through gravimetric analysis where the mass variation in the COMPS over time was monitored. To develop a COMPS a permeable polymer bag was used to put the material in. In the past, LDPE have shown less variance between the porosity/dissipation rates of the COMPS and was therefore used as the inner bag for the construction of COMPS (103). While if aids were transported to the 
field, the inner bag was then placed inside a non-permeable secondary container (e.g. mason jar) to preserve the odor and to avoid contamination. Previous research have shown that the best secondary containers are mason jars as they provide a reliable sealing which avoid humidity and reduce the dissipation over time in comparison with other packages to 94\% (103). COMPS varying in absorbent material were analyzed over a series of weeks from which the dissipation rate was determined. This study shows how the complexity of a polymer determines the trapping capabilities of a compound over time. In Figure 28 and 29 it is suggested that alumina have the capacity of retaining terpenes better than cellulose which decreases in weight faster in a period of two weeks.

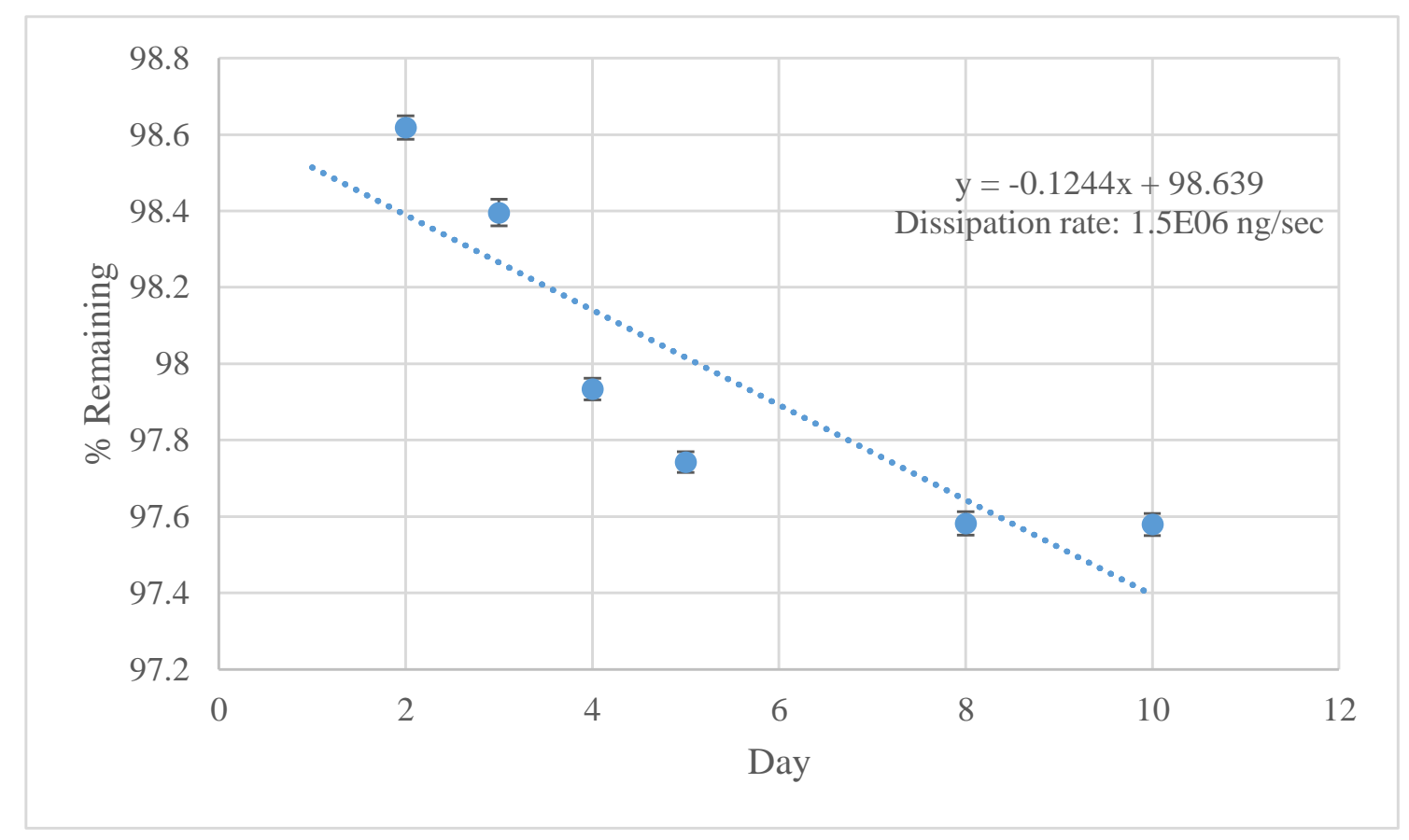

Figure 28. Cellulose based training aid mimic for marijuana 


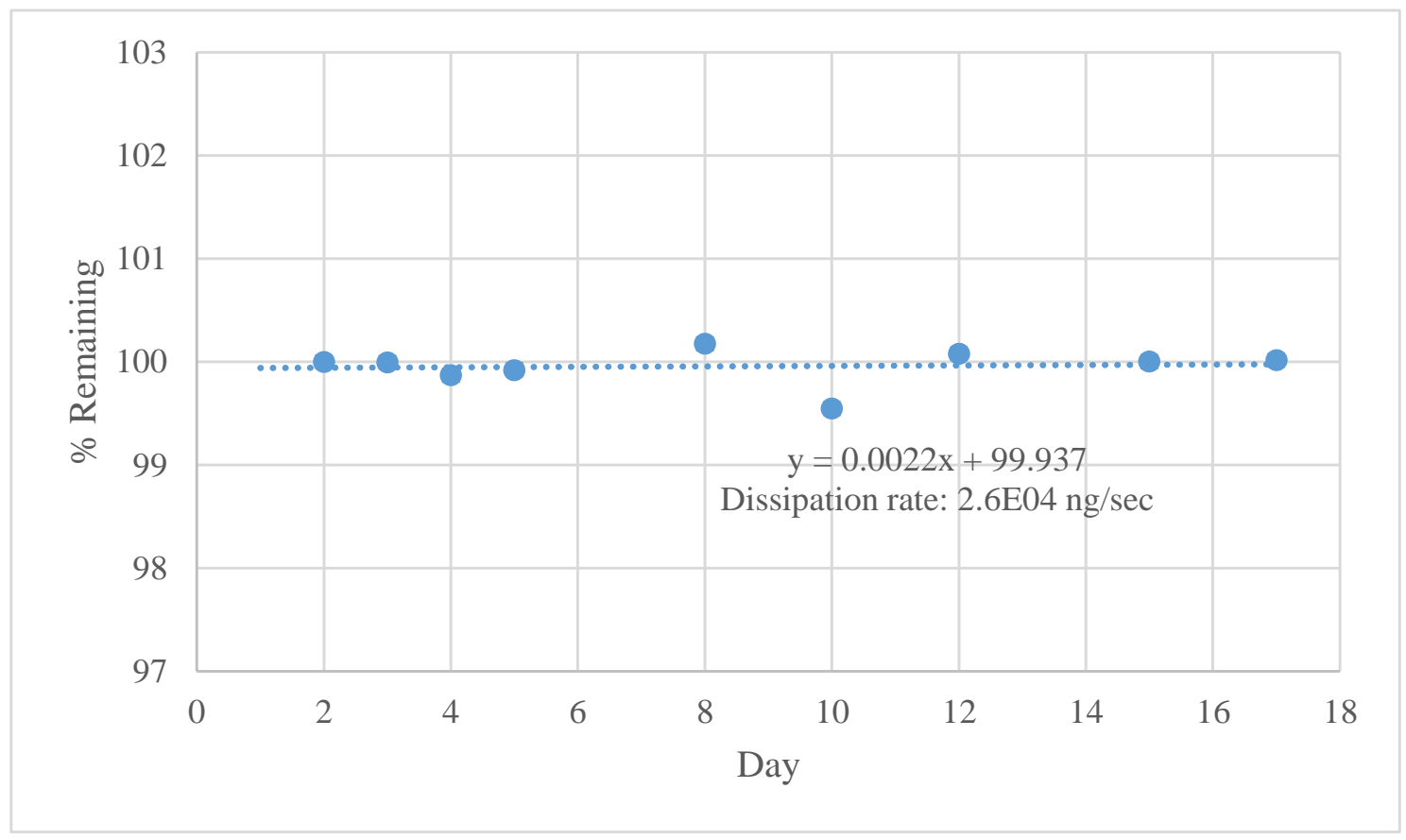

Figure 29. Alumina Based Training Aid Mimic for Marijuana

\subsubsection{Heroin}

When evaluating the weight of the pseudo heroin COMPS, the data was not consistent therefore, a most rigorous approach was considered. The headspace of the samples was now been evaluated, but the data did not followed a trend over time. The reason for such inconsistency could be explained by how environmental conditions or even the frequency at which fibers are used for extraction have a direct impact on the stability of compounds. However, there are sample preparation procedures that can be used to account for the changes that otherwise could not be reduced. One of the most powerful approaches used to reduce uncertainty is the internal standard (IS). An ideal IS will be that one with similar structural characteristics as the analyte and exhibit analogous behavior in the analysis scheme, but most importantly that is not present in the sample (158). To normalize the 
results obtained via HS-SPME MacCrehan have suggested a new approach where the "internal standard" is present on the fiber rather than in the sample itself. This approach is known as externally sampled internal standard (ESIS) and will be used for the dissipation rate study of acetic acid. For the analysis the ratio between the analyte (A) and ESIS (E) will be calculated as follows:

$$
\frac{A}{E}=\frac{\text { peak area of analyte }}{\text { peak area of ESIS }}
$$

\section{Equation 4}

But first, an "external standard" was selected based on the characteristics of acetic acid; hexanal $98 \%$ pure (Sigma-Aldrich, Co., St. Louis, MO, USA) as it contain close geometric structure and vapor pressure (159).

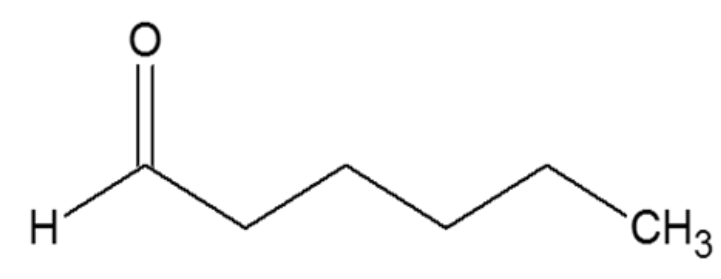

A
VS

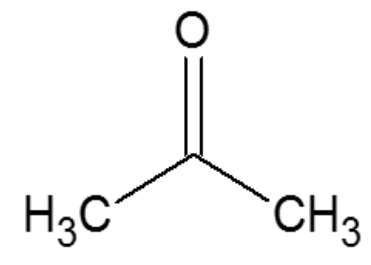

$\mathrm{B}$

\section{Figure 30. (A) Hexanal 10 mmHg @ $20{ }^{\circ} \mathrm{C}$ (B) Acetic Acid $11.4 \mathrm{mmHg} @ 20{ }^{\circ} \mathrm{C}$}

Once the external standard was selected, an optimization of the optimum SPME equilibrium/extraction was performed from which the ideal parameters were found to be 45 min equilibrium followed by $45 \mathrm{sec}$ extraction. Headspace equilibrium of both analyte and ESIS was achieved in the first 45 min of sampling where the ratio kept constantly 
below 1.0 while later over time the increase in ratio was abrupt. This suggest that short times did not allow for fiber absorption competitions while when more time was allowed there was either competition for the sites in the fiber or the detector response was saturated with the high amount of material.

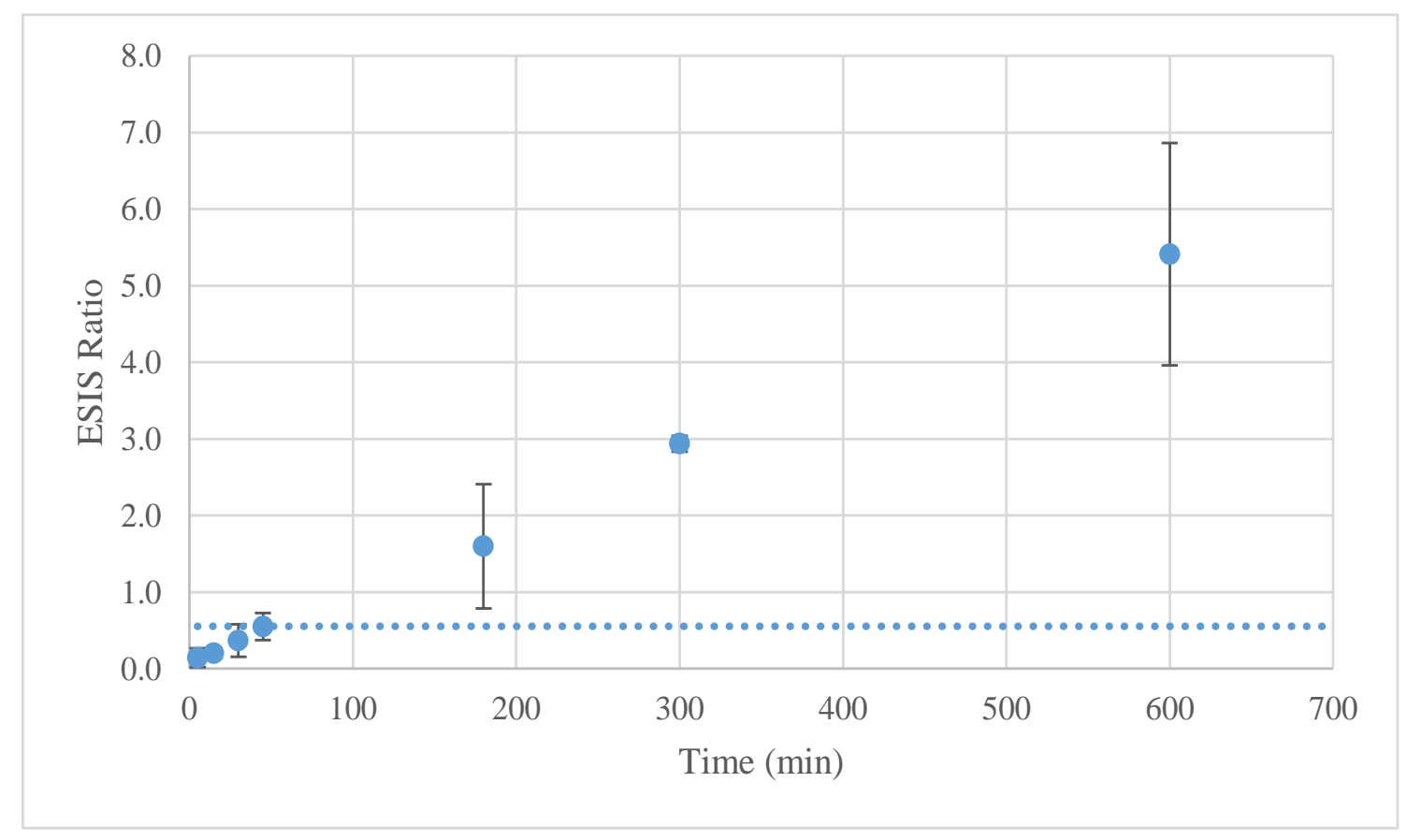

Figure 31. Assymetrical ESIS (Hexanal)-Acetic Acid

Once parameters for the ESIS were established SPME-ESIS was used to compare the amount of odor released by the training aids over time. Overall, triplicates of the training aids were evaluated daily utilizing the same fibers for the period were VOCs were detectable. As depicted in Figure 31, the volatile (160) was slightly released over time. Despite its decrease, the use of ESIS demonstrated a lower relative uncertainty by a factor of 2 when compared to the inconsistency previously obtained when raw SPME/GC/MS was used to determine the dissipation over time. The data collected showed that the odor 
was detected for a period of two weeks. However, as it has been demonstrated that detector canines are more sensitive than an analytical instrument, further field trials must be conducted to determine if in fact, the training aid can be detected by canines for longer periods of time. This way once more it could be demonstrated the capabilities of canines as a detection tool.

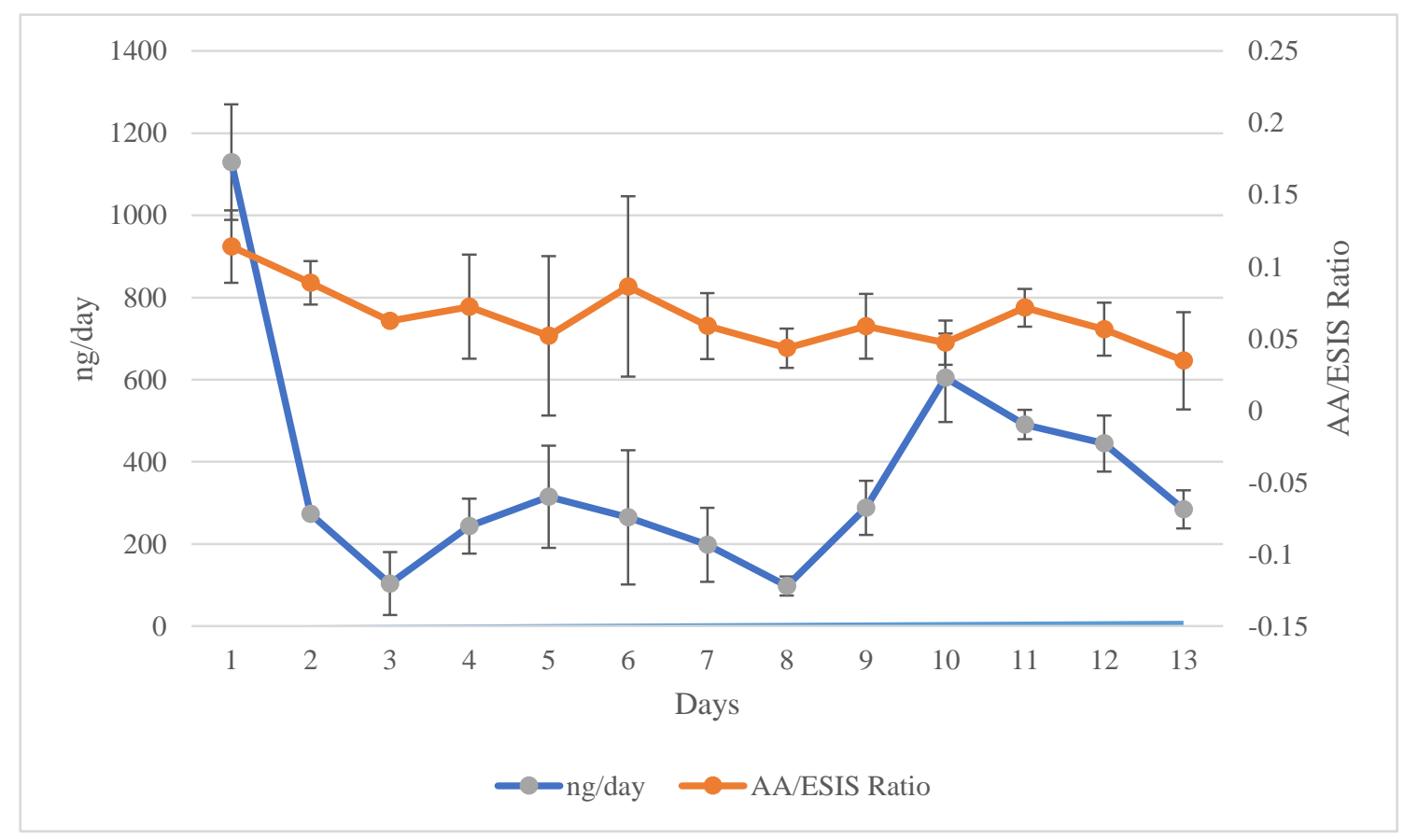

Figure 32. Pseudo Heroin Dissipation Over Time

\subsection{Conclusion}

When a training aid is being developed there are several parameters that need to be considered for the maintenance of potency, efficacy, and functional integrity of a canine. Although previous research have suggest suitable storage parameters such as the absorbent material efficiency, depends on the target chemical been used a training aid. The study of the dissipation rate can be performed using gravimetric analysis, but when data does not 
follow a trend over time, a more precise approach need to be considered. Moreover, as changes in temperature and moisture have a huge impact on both the strength of odors and also how quickly they dissipate, both parameters were monitored over time. Pseudo marijuana compounds were analyzed using gravimetric analysis. Results over time suggest a steady dissipation over a week period when alumina is used as the absorbent material.

On the other hand, pseudo heroin target compounds stability were greatly affected by variations in humidity and temperature impact in the laboratory. Therefore, the headspace of this training aid were analyzed using ESIS (hexanal) which recompense for these changes and a more accurate data set was obtained. Absorbent materials such as cellulose and alumina were evaluated to determine the longevity effect as response to different materials. Cellulose creates a more complex polymer chain, having thus less space between molecules which creates a better trapping source for the target compounds therefore, when compared to alumina, molecules in cellulose were retained for a longer time (2 weeks).

When the training aid are taken to the field, aids must be always stored at room temperature. If exposed to extreme temperatures, the permeation of the odor may not be constant, therefore if training on a day where temperatures are high, the aid will have a faster permeation but when training on low temperatures day, the permeation will be reduced. 


\section{TASK 5. FIELD TRIAL VALIDATION OF NEWLY DEVELOPED TRAINING AIDS}

\subsection{Introduction}

The maximum proficiency level of detector canines greatly depends on the frequency of training. For many years, training has been limited due to a lack of training materials that are easy to acquire, low priced, non hazardous, long lasting, but most important, effective. A prototype of training aid mimic for marijuana and heroin have been developed to alleviate this restrictions. Training aid mimics were developed after thoroughly analyzed the most frequent and abundant VOCs released by each drug. When dealing with training aid mimic, aspects such as integrity, purity and availability of odor must be considered. As indicated by SWGDOG SC8 2007, the best practice to increase persistence and avoid contamination were followed, which include the storage of training aids in separate containers such as stainless steel or glass, preferable with lids to avoid any sort of air exchange. The purpose is now to proof these aids are feasible as training aids for detection canines, which are meant to be used as a universal system to improve and keep consistent work done. Additionally, the fact that training aids may contain compounds that are found in comestible and commercially available products has always been controversial in the court of law therefore, ORTs including these products were conducted to validate training aid mimics are not product that could potentially cause false alerts. Field studies were performed using detection canines that have been previously imprinted to detect real marijuana and heroin. These canines have also proof their capability for detection by certifying annually under SGWDOG's guidelines which implements real scenarios simulations to which teams can be exposed to. Experienced detection canines used in this 
study have previously shown proficient narcotic detection skills at a detection rate of $90 \%$ or higher. Canines that were still in training school or that have obtained less than $90 \%$ detection rate were not allow to participate.

\subsection{Materials}

Training aid mimics were developed following the standard procedure recently developed. Two different absorbent materials were used, alumina adsorption 80-100 mesh (Fisher Scientific, Fair Lawn, NJ, USA) for marijuana training aid mimic and cellulose microcrystalline powder $20 \mu \mathrm{m}$ (Sigma-Aldrich, Co., St. Louis, MO, USA) for heroin training aid mimic. For marijuana training aid, $30 \mathrm{~g}$ of alumina were spiked with a mixture of the limonene and caryophyllene (Sigma-Aldrich, Co., St. Louis, MO, USA). On the other hand, 145.5 $\mu \mathrm{L}$ of acetic acid (Fisher Scientific, Fair Lawn, NJ, USA) and $288 \mathrm{mg}$ of acetyl salicylic acid (Sigma-Aldrich, Co., St. Louis, MO, USA) were added to the adsorption materials for heroin mimic. These amounts were selected based on the ratio found in the headspace of each sample. Upon completion, the mixtures were shaken in their corresponding mixing jars utilizing United Nuclear Ball Mill (United Nuclear Scientific, East Lansing, MI, USA) for $5 \mathrm{~min}$ every $30 \mathrm{~min}$ for $3 \mathrm{hr}$ to achieve an homogeneous mixture of odorant. Once the mixture was ready, it was divided into 3 LDPE bags containing $10 \mathrm{~g}$ each, these bags were later heat sealed three times using an Midwest Pacific impulse heat sealer (Packco Inc., Rocky Mount, MO, USA). The bags were later

transferred to $8 \mathrm{oz}$ Ball® Crystal Jelly Jars with Lids and Bands (Ball, Co., Broomfield, CO, USA). 


\subsection{Methods}

To resemble normal operational conditions, trials were performed in warehouses and/or buildings. The Scientific Working Group on Dog and Orthogonal detector Guidelines (70) was followed as it establishes the best practices for the use and evaluation of detector canines. SC8 Substance Dogs: Narcotics establishes that when evaluations in Building/room is been performed the room may contain zero to three aids depending upon the size and environmental conditions, and should be $18.6-111.5 \mathrm{~m}^{2}$ (200-1200 sq. ft.) with furniture. Specifics on the buildings/room where the trials were performed can be found in the Appendix section. Training aids (hides) were placed no less than 30 minutes prior to testing. In order to demonstrate the ability to detect the trained odors, positive control (132) and a blank were always included. Although the handlers were advised of the parameter of search, teams did not know the placement of the hides. Handlers were only allowed to reward the canine when they positive alerted to the positive control otherwise alerts were only recorded.

\subsection{Results and Discussion}

\subsubsection{Marijuana}

Canine teams from South Florida participated in three different ORTs that varied in location, date and hides included. The handlers were instructed to have their canines search for hides within a specified perimeter, observations including alert, no-alert, or interest were recorded. The change of behavior towards a specific location without giving a final response was considered for the study as interest, but was only recorded following the handler's indication, as they have more experience regarding canines' change of behavior 
when searching. The total number of trials per canines is represented in the denominator of the fraction while the number of alerts was recorded in the numerator. Interests were recorded and reported in the table, in parenthesis.

Table 16. Individual Canine Performance Table on Pseudo Marijuana Training Aid (Numerator $=$ A, Denominator $=$ No alert, Parenthesis $=$ Interest $)$

\begin{tabular}{cccc}
\hline Canine No. & $\mathrm{A}$ & $\mathrm{C}$ & $\mathrm{PC}$ \\
\hline 30 & $0 / 1$ & $0 / 1(1)$ & $1 / 1$ \\
12 & $0 / 1$ & $1 / 1$ & $1 / 1$ \\
22 & $1 / 1$ & $0 / 1$ & $1 / 1$ \\
29 & $1 / 1$ & $0 / 1$ & $1 / 1$ \\
31 & $1 / 1$ & $0 / 1$ & $1 / 1$ \\
21 & $1 / 1$ & $1 / 1$ & $1 / 1$ \\
\hline
\end{tabular}

Note: $\mathrm{A}=30 \mathrm{~g}$ of marijuana mimic on alumina, $\mathrm{C}=30 \mathrm{~g}$ marijuana mimic in cellulose, $\mathrm{PC}=100 \mathrm{~g}$ positive control (marijuana)

As depicted in Table 17, every canine successfully alerted to the positive control (marijuana) which proof the discriminatory proficiency skills of the canines who participated in the study. However, only $66.6 \%$ of the canines alerted to the mixture suspected to be responsible for the alert response of marijuana when alumina was used as an adsorbent material. When this type of study is performed, a $90 \%$ or higher combined rate of detection is preferable to validate the training aid mimic. Therefore, it is required to manipulate the recipe to increase the number of detection rate. The fact that the mimic is for a natural harvested product rather than synthetic explain the fact that is not a straightforward procedure. Although marijuana plants can be identical in color and shape it is not correct to assume all of them have the same odor profile. The fact that there is a 
diverse variation of plants that differ in cultivation procedures might be changing the relative abundance and interaction of the volatiles which in turn might affects the odor profile. Also, note that there are marijuana plant genders, strains and species (24) that are only controlled by the grower, therefore to better understand the difference of the VOCs makeup of marijuana, more information from the origins of the sample is needed.

Table 17. Odor Recognition Test (Average): Pseudo Marijuana (n=6)

\begin{tabular}{ccccc}
\hline Training Aid & Alert Rate $(\%)$ & Interest Rate $(\%)$ & $\begin{array}{c}\text { No Alert } \\
\text { Rate }(\%)\end{array}$ & $\begin{array}{c}\text { Combined Rate of } \\
\text { Detection }(\%)\end{array}$ \\
\hline A & 66.6 & 0.0 & 33.3 & 66.6 \\
C & 33.3 & 16.6 & 50.0 & 50.0 \\
PC & 100.0 & 0.0 & 0.0 & 100.0 \\
\hline
\end{tabular}

Based on the canines responses listed on Table 17 PPV and NPV values were calculated. The PPV value for the marijuana training aid mimic in alumina was $43 \%$. This value means that $43 \%$ of the canine alerts were correct. In contrast the NPV was $60 \%$, meaning that meaning that $60 \%$ of the time a canine did not give a response, when there was nothing hidden thus, the non-response was correct. The quality of the training aid mimic and the proficiency of canines are been dictated by the correct positive alert (PPV). If instead, canines give a correct non-response (NPV) could possibly be caused by the use of contaminated or ineffective training aid mimic (does not contain an odor of interest). A PPV of $43 \%$ indicates that odors from the training aids created in this study were reasonably recognizable to narcotic canines.

The second set of ORTs included the evaluation of comestible products to discard the idea that canines might alert to this sort of products as some contain the target chemical(s) 
used as training aid mimics. As shown in Figure 33, when $1 \mathrm{~g}$ of lemon peel was extracted for 1 min using DVB/CAR/PDMS SPME fiber, limonene was detected to be the greatest contributor of the headspace. When the abundance of the peak in lemon peel was compared to limonene in marijuana results reflected that lemon peel releases $210 \%$ of what marijuana does. Black pepper was also evaluated, as seen in the figure, in this case both limonene and caryophyllene were obtained from the headspace. But when compared to the same terpenes in the headspace of marijuana, the relative abundances are significantly different. Limonene is $55 \%$ and caryophyllene is $12 \%$ of what is found in the headspace of marijuana. 


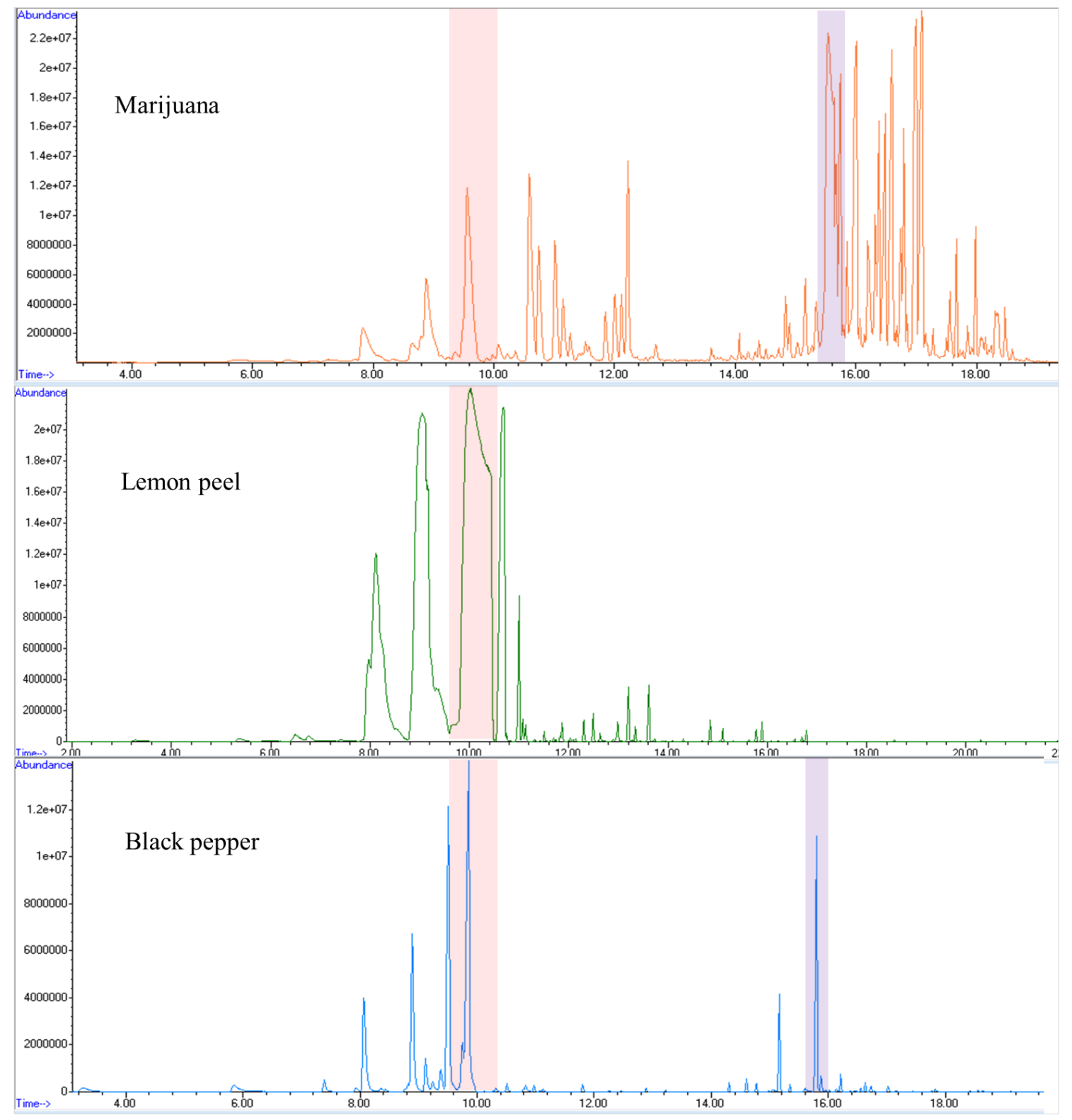

Figure 33. Headspace of Marijuana and Different Comestible Products Containing Limonene (Red) and/or Caryophyllene (Purple) as a Main Chemical Compound

By performing a set of ORT, the discriminatory power of canines was proven. Although substances may share some compounds it does not implicate that this substances will increase the false alert rate when present in a perimeter while searching for illicit materials. Canines are known to discern between a single compounds versus a pool of 
compounds that makes up the headspace of the substance. When comestible products were included as hides for search none of the canines alerted or showed interest.

Table 18. Individual Canine Performance Table on 1 g Comestible Products $(n=5)$ (Numerator $=A$, Denominator $=$ No alert, Parenthesis $=$ Interest $)$

\begin{tabular}{cccc}
\hline Canine No. & PC & Lemon peel & Black pepper \\
\hline $\mathbf{3 1}$ & $1 / 1$ & $0 / 1$ & $0 / 1$ \\
$\mathbf{2 1}$ & $1 / 1$ & $0 / 1$ & $0 / 1$ \\
$\mathbf{1 3}$ & $1 / 1$ & $0 / 1$ & $0 / 1$ \\
$\mathbf{3 0}$ & $1 / 1$ & $0 / 1$ & $0 / 1$ \\
$\mathbf{3 5}$ & $1 / 1$ & $0 / 1$ & $0 / 1$ \\
\hline
\end{tabular}

The significant difference between the odor profiles of the lemon peel, black pepper, and marijuana, and the fact that no alert response or interest was obtained, supports the canines' ability to differentiate between comestible products and marijuana, even if the target odor is present in any samples. Additionally, the fact that canines alerted to the positive control (marijuana) shows how proficient detector canines are when performing their work.

Table 19. Odor Recognition Test (Average): Comestible Products $(\mathbf{n}=5)$

\begin{tabular}{ccccc}
\hline Hide & Alert Rate $(\%)$ & Interest Rate $(\%)$ & $\begin{array}{c}\text { No Alert } \\
\text { Rate }(\%)\end{array}$ & $\begin{array}{c}\text { Combined Rate of } \\
\text { Detection }(\%)\end{array}$ \\
\hline PC & 100.0 & 0.0 & 0.0 & 100.0 \\
Lemon peel & 0.0 & 0.0 & 0.0 & 0.0 \\
Black pepper & 0.0 & 20.0 & 0.0 & 0.0 \\
\hline
\end{tabular}




\subsubsection{Heroin}

Canines from different law enforcement officers in South Florida participated in various ORTs that varied in location, date and quantity of aids hidden. The handlers were instructed to have their canines sample the odor in each scenario and identify a response of alert, no-alert, or interest. The change of behavior towards a specific location without giving a final response was considered for the study as interest, but was only recorded following the handler's indication. The total number of trials per canines is represented in the denominator of the fraction while the number of alerts was recorded in the numerator. All canines alerted to the heroin mimic samples regardless of the quantity, location and/or environmental conditions, indicating the proficiency of their work.

Table 20. Individual Canine Performance Table on Pseudo Heroin Training Aid (Numerator $=$ A, Denominator $=$ No alert, Parenthesis $=$ Interest $)$

\begin{tabular}{cccccc}
\hline Canine No. & $20 \mathrm{~g}$ & $10 / 30 \mathrm{~g}$ & $30 \mathrm{~g}$ & $20 \mathrm{~g}$ & $30 \mathrm{~g}$ \\
\hline 12 & $2 / 2$ & $2 / 2$ & - & - & $1 / 1$ \\
19 & $2 / 2$ & - & - & - & - \\
29 & $2 / 2$ & - & $1 / 1$ & $1 / 1$ & $1 / 1$ \\
31 & $2 / 2$ & - & $1 / 1$ & $1 / 1$ & $1 / 1$ \\
22 & $2 / 2$ & $2 / 2$ & $1 / 1$ & $1 / 1$ & $1 / 1$ \\
30 & $2 / 2$ & $2 / 2$ & $1 / 1$ & - & - \\
27 & $2 / 2$ & & - & - & - \\
21 & $2 / 2$ & $2 / 2$ & - & - & - \\
13 & $2 / 2$ & $2 / 2$ & - & - & $1 / 1$ \\
34 & - & - & - & $1 / 1$ & - \\
24 & - & - & - & $1 / 1$ & - \\
35 & - & - & - & - & $1 / 1$
\end{tabular}


Real heroin was also included as a hide in the search to which all canines successfully alerted. A $90 \%$ or higher combined rate of detection is preferable for novel training mimic and since all of the heroin mimics achieved this response (Table 21), the aids could be accepted as a valid odor to be included along with the drugs mimics that have already been validated.

Table 21. Odor Recognition Test (Average): Pseudo Heroin (n=12)

\begin{tabular}{ccccc}
\hline Training Aid & Alert Rate $(\%)$ & Interest Rate $(\%)$ & $\begin{array}{c}\text { No Alert } \\
\text { Rate }(\%)\end{array}$ & $\begin{array}{c}\text { Combined Rate of } \\
\text { Detection }(\%)\end{array}$ \\
\hline $10 \mathrm{~g}$ & 100.0 & 0.0 & 0.0 & 100.0 \\
$20 \mathrm{~g}$ & 100.0 & 0.0 & 0.0 & 100.0 \\
$30 \mathrm{~g}$ & 100.0 & 0.0 & 0.0 & 100.0 \\
PC & 100.0 & 0.0 & 0.0 & 100.0 \\
\hline
\end{tabular}

Note: $\mathrm{PC}=$ positive control (heroin)

To support the validity of training aid mimic developed PPV and NPV values were calculated based on the canines responses in Table 21 . The PPV values was $100 \%$, in other words, this value indicates that all canines correctly alerted when in fact there was a training aid mimic hidden. The correct positive alert (PPV) value therefore, highlights the efficiency of the training aid mimic as suitable alternative for detection canine. On the other hand, canines gave a 100\% correct non-response (NPV) which also explains the success training aid mimic (does contain an odor of interest) and the fact that if training aids are storage appropriately contamination of these are the least of concerns. A PPV of $100 \%$ indicates that odors from the training aids created in this study were reasonably recognizable to narcotic canines. 
The second set of ORTs included the evaluation of comestible and commercially available products that are known to contain VOCs such as acetic acid and acetic salicylic acid. For instance, acetic acid is commonly used for food preservation as what is known as distilled vinegar which is essentially diluted synthetic acetic acid (160). On the other hand there is aspirin which is synthesized from salicylic acid using the same chemical compound that is used for the synthesis of heroin; acetic anhydride and conversely releases acetic acid to the headspace. The previous statements were confirmed instrumentally by adding $1 \mathrm{~g}$ of each product to a $10 \mathrm{ml}$ glass vial that was later tightly closed with a cap containing a septum. Triplicates extractions were performed for 1 min using DVB/CAR/PDMS fiber as were used for heroin samples. 

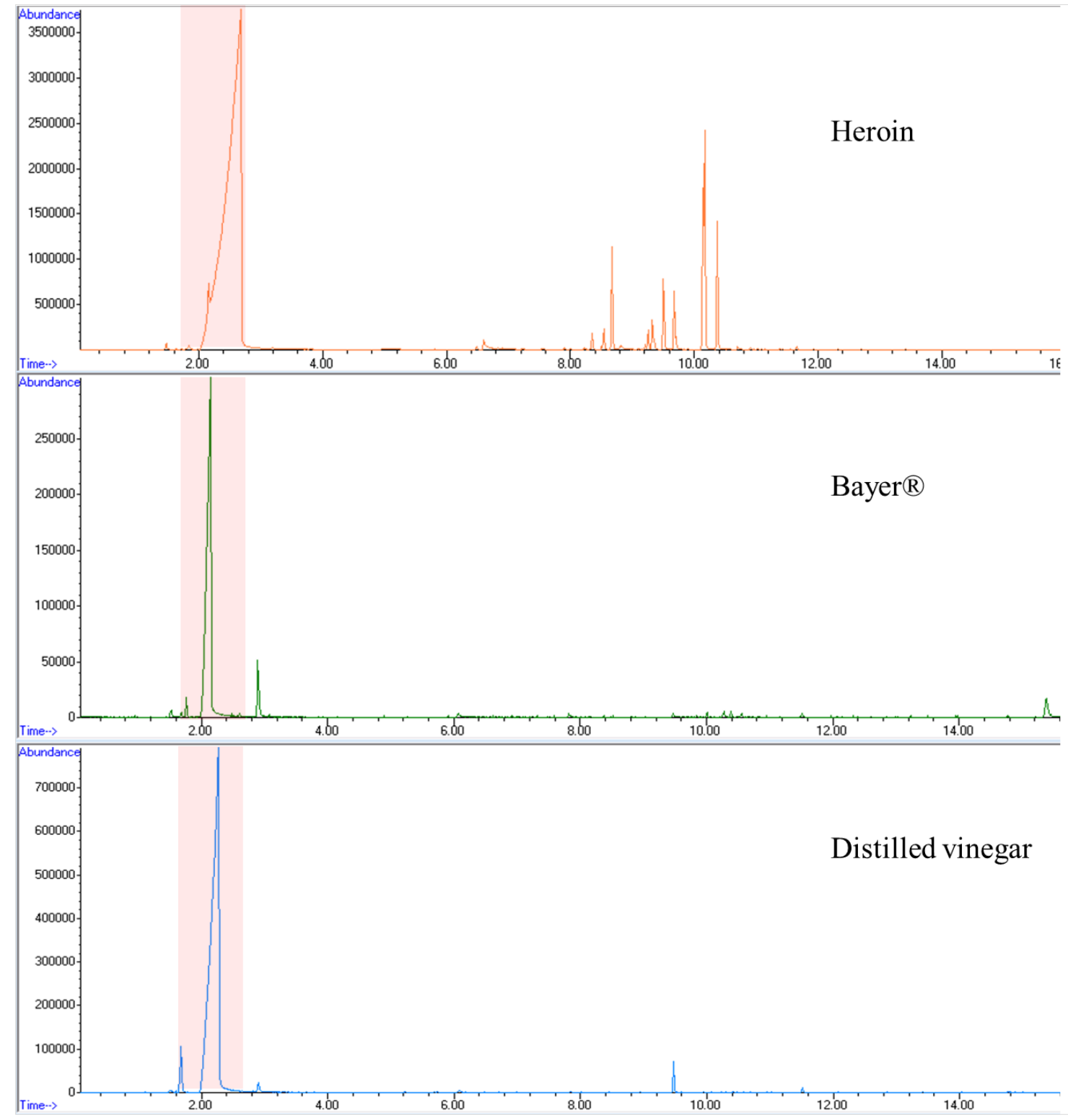

Figure 34. Headspace of Heroin and Different Commercially Available Products Containing Acetic Acid (Red) as a Main Chemical Compound

As depicted in Figure 34, although all the products analyzed had acetic acid in common the percentages in comparison with heroin where significantly different. Bayer ${ }^{\circledR}$ caplets only contained $2 \%$ of the acetic acid contained in heroin while distilled vinegar contained 
$11 \%$ of what heroin has. When ORTs were performed, none of the canines but one showed interest (a quick change of behavior after passing by the hide the first time).

Table 22. Individual Canine Performance Table on Commercially and Comestible Products $(\mathbf{n}=5)($ Numerator $=A$, Denominator $=$ No alert, Parenthesis $=$ Interest $)$

\begin{tabular}{cccc}
\hline Canine No. & PC & Bayer ${ }^{\circledR}$ & Distilled vinegar \\
\hline $\mathbf{3 1}$ & $1 / 1$ & $0 / 1$ & $0 / 1(1)$ \\
$\mathbf{2 1}$ & $1 / 1$ & $0 / 1$ & $0 / 1$ \\
$\mathbf{1 3}$ & $1 / 1$ & $0 / 1$ & $0 / 1$ \\
$\mathbf{3 0}$ & $1 / 1$ & $0 / 1$ & $0 / 1$ \\
$\mathbf{3 5}$ & $1 / 1$ & $0 / 1$ & $0 / 1$ \\
\hline
\end{tabular}

Canines' ability to discriminate between a single odor versus a pool of odor containing traces of the target odor is a subject that is continuously brought when validity of canines performance is debated (119). This set of recognition test suggest that the amount of target odorant, and what make up the headspace of a substance does play an important role in regard to what a canines alerts to. The significant difference between the odor profiles of the Bayer®, distilled vinegar, and heroin, suggests that the pool of odor released from Bayer ${ }^{\circledR}$ or vinegar supports the canines' ability to differentiate between commercial products and heroin, even if the active odor is present in both samples. Thus, the fact that several commercially available product may contain chemicals associated with the target compound(s) in training aids does not implicate that canines will alert to every product that releases it to the headspace. 
Table 23. Odor Recognition Test: Commercial and Comestible Products (n=5)

\begin{tabular}{ccccc}
\hline Hide & Alert Rate $(\%)$ & Interest Rate $(\%)$ & $\begin{array}{c}\text { No Alert } \\
\text { Rate }(\%)\end{array}$ & $\begin{array}{c}\text { Combined Rate of } \\
\text { Detection }(\%)\end{array}$ \\
\hline PC & 100.0 & 0.0 & 0.0 & 100.0 \\
$\begin{array}{c}\text { Bayer@ } \\
\text { Distilled } \\
\text { Vinegar }\end{array}$ & 0.0 & 0.0 & 0.0 & 0.0 \\
\hline
\end{tabular}

\subsection{Conclusion}

Field trial validation was used to establish documented evidence to provide a high degree of assurance of training aid quality and functionality by demonstrating canines consistently produce the desired response. Therefore, field trial validation tests were performed as ORTs to prove how feasible training aids are for detection canines. Training aid mimics are meant to be used as a universal system to improve and keep consistent work done when real drugs are not available. Although limonene and caryophyllene have both been suggested to be two of the most odorous components in marijuana samples (132), when the marijuana training aid mimic, composed of a 1:1 mixture of limonene and caryophyllene, was hidden for certified detection canines to search, $66 \%$ either alerted or showed interest. Since marijuana is a natural harvested product, there are variables that were not considered due to unavailability on origins information. Although plants can be identical in color and shape they may not produce the same odor because of the large diversity of volatile compounds, and their relative abundance and interactions that vary depending on where it is grown and the environmental conditions associated with it (161). In the past a mixture of $50 \mu \mathrm{L}$ each of five compounds: $\alpha$-pinene, $\beta$-pinene, myrcene, limonene, and $\beta$-caryophyllene was presented to canines for detection but no alert was 
accomplished (9). It has also been suggested that caryophyllene could be responsible for the alert response of canines but when this compound by itself was tested, less than $90 \%$ was also obtained. Therefore, modification on the ratios, recipe and compounds that are not terpenes but may contribute to the odor released from marijuana must be considered. Now, different comestible products known to release similar terpenes as marijuana were tested to discard the statement that canines may alert to comestible products since some share the same compounds as the illicit substance. But canines discrimination power is high enough to differentiate between a single compound and a pool of compound containing the target chemical. For this reason, when canines' proficiency was evaluated, none of the canines who participated demonstrated any sort of interest on lemon peel or ground pepper both known to release terpenes.

The same approach was considered for heroin. To validate the training aid mimic for heroin, a set of ORTs varying in the location, date, and amount of training aid, were performed to demonstrate the functionality when presented to canines. Thirty grams of heroin training aid mimic were separated into 3 bags containing $10 \mathrm{~g}$ each. To simulate canine training scenarios, different amounts of heroin training aid mimic were presented to the canines. When searching, detector canines showed no problem in finding the aids regardless of the amount (10, 20 or $30 \mathrm{~g})$ over a 9 week period where the training aids were never changed but kept in a $8 \mathrm{oz}$ jar to avoid any sort of contamination and to preserve the odor. Out of 12 canines, an average of $100 \%$ alerted to the aid as well as the positive control (heroin). This demonstrates that the combination of odorants suggested to be responsible of the alert response from canines have been satisfactory. Also, to demonstrate that the use of a target compound that is shared by commercially available product does not implicate 
that canines will false alert, a second set of ORTs were performed. Bayer ${ }^{\circledR}$ and distilled vinegar were tested for this purpose and no canines alerted to any of them while only one canines showed interest. This proves once again, canines' field accuracy and discrimination power.

\section{TASK 6. TRAINING AIDS DEVELOPMENT ALTERNATIVE FOR NEWLY DESIGN SUBSTANCES}

\subsection{Introduction}

Despite advantages of instrumental detection to date, canines continue to be an essential and primary source for the rapid detection of explosives since there is nothing as yet that compares to canines' olfactory capabilities $(7,8,162)$. The growing threat of improvised explosives has created a worldwide concern and emphasized the requirement of a greater spectra of training aids that cover a wider range of currently available explosives. High sensitive methods that have potential to alleviate this issue are observed in the area of remote detection of explosives $(44,127)$. In remote detection, the personnel performing explosives screening maintain a safe distance from the item being screened, but the screening equipment does not (128). The technique is more commonly applied for explosive scent tracing in landmines and has witnessed a fruitful area of research. The uses of various pumping systems to extract the air above suspected explosive-containing substance has allowed these samples to then be presented to trained canines (90) for detection purposes (163). Based on the biological detector response, responders can then search more thoroughly for security controls. Of importance in this line of work then, is the concept of optimal odor availability in any given situation when performing these sampling methods $(164,165)$. It is crucial to effectively optimize the collection method in 
addition to monitor and measure the amounts of detectable explosive vapors in remote sampling schemes and further yet monitor confinement in terms of dissipation and persistence rates. Very little work has investigated how long a target explosive volatile lasts on the collection medium after the material has been exposed via a non-contact approach to the explosive odor source.

The collection device in this study is a non-commercially available prototype that includes a user-friendly interface, a durable lightweight body, internal power supply and a design to minimize cross contamination. The system includes a leaf attached mechanism, allowing the user to open and close the attachment to hold the gauze pad in place. The device contains a digital display that allows the user to monitor the battery life, control the airflow speed and collection time by navigating the selection menu using a keyboard. The device is a portable vacuum that, when swept over the explosive of interest, draws air toward the device, collecting any volatile organic compounds (VOCs) present onto the adsorbent material. The collection material can then be potentially presented to the canine for training or returned to a laboratory for analysis. The ease of use and portability of the system allows for its deployment in field operations, which yields to the potential expansion of its use in other areas of critical need for homeland security and national interest. The use of a portable non-contact collection system seeks to facilitate the creation of training aids not only for those explosives that are currently available but also for the ever evolving explosive recipes encountered in military operations.

The exact composition of canine training aids is a pivotal area in optimal explosive detection efforts and has thus seen an active growth in research $(56,166-168)$. The target compounds considered unique to identify each explosive family are detailed in (Table 24). 
Depending on the volatility and chemical structure is the ease of collection however, after the collection the persistence of odor is known to change over the time scale of minutes (14). Therefore, dynamic monitoring of these compounds can help determine the dissipation of the scent but also, how different storage containers impact the preservation of odor after having been dynamically collected.

Table 24. Target Compound per Explosive Family

\begin{tabular}{lll}
\hline Explosive family & Target compound & Formula \\
\hline Peroxides & Triacetone triperoxide & TATP \\
Acid salts & Carbamate derivative & AN \\
\hline
\end{tabular}

Characteristics of ideal training aids include: inertness for safe handling, harmlessness to the canine; generation of detectable levels of key odorant(s) with high effectiveness relative to real explosives and possesses suitable longevity with minimal storage considerations $(15,20,21)$. Thus, optimization of the collection method will be assessed in addition to the evaluation of storage containment systems of the VOCs. The purpose of this study is the to optimize the non-contact collection method, to evaluate a variety of storage containment systems, and to determine how these affect the preservation of target explosive odor samples over time. The optimal storage container and preservation time range will be determined based on the least amount of background as well as the maximum odor contained in any given sample after instrumental analysis. 


\subsection{Materials}

Dukal ${ }^{\circledR}$ sterile 4” x 4" gauze pads (Dukal Corporation, Ronkonkoma, NY) were used as the collection material. Compounds used as target chemicals to monitor the persistence over time included: TATP (The Bureau of Alcohol, Tobacco, Firearms and Explosives, ATF) and ammonium nitrate (Acros Organics, Morris, NJ). The derivatizing reagent for AN was n-butylchloroformate (Sigma-aldrich, St. Louis, MO). Three different storage containers will be evaluated to determine which one preserves samples the best, these are: Ziploc ® 1.2 Mil resealable sandwich bags (Johnson \& Son, Inc., USA), 6” x 5.5” ID Barrier foil ziplock bags (Ted Pella, Inc., Redding, CA), and $40 \mathrm{~mL}$ clear glass screw top vials with Polytetrafluoroethylene (PTFE)Silicone septa (Sigma-aldrich, St. Louis, MO). For the extraction of target compounds, the fibers used for solid phase microextraction (SPME) were polydimethylsiloxane/Divinylbenzene (PDMS/DVB) and polyacrylate (PA) (Supelco, Sigma-aldrich, St. Louis, MO).

\subsection{Methods}

Departing from the premise that ammonium nitrate (AN) is an inorganic salt having thus a low volatility, it is fundamental to note that unless derivatization is conducted the compound is not possible to be detected by gas chromatography mass spectrometry (GCMS). Derivatization is the process by which a compound is transformed into a new compound amenable to the analysis. Therefore, for the analysis of $\mathrm{AN}$, a derivatization was performed using n-butylchloroformate as a derivatizing reagent. Sample preparation was needed thus; based on Brown et al. published studies for the analysis of ammonium based explosives using SPME-GC/MS, a solution of ammonium nitrate in water $(1 \mathrm{mg} / \mathrm{mL})$ was 
treated with equal quantity of a solution of sodium hydroxide in water $(1 \mathrm{mg} / \mathrm{mL})$. Upon completion of the sample preparation, Polyacrylate (PA) SPME fiber was exposed for $30 \mathrm{sec}$ to the headspace of the derivatizing reagent followed by the exposure of the fiber to the analyte solution for 20 minutes. After derivatization process was completed, the sample was desorbed in the GC-MS for $5 \mathrm{~min}$.

The Bureau of Alcohol, Tobacco, Firearms and Explosives (ATF) provided three samples of individually sealed peroxide based explosive triacetone triperoxide (56). Each pack included a sniffer tin containing $7 \mathrm{mg}$ of TATP dried on a glass microfiber pad. After the samples were received they were immediately placed in the refrigerator at $2^{\circ} \mathrm{C}$ in order to preserve the integrity of the samples. To ensure the quality of the explosive samples, the headspace of each was analyzed using SPME prior to the sampling with the dynamic collection system. Samples were placed in $8 \mathrm{oz}$ glass jars for the extraction of VOCs from the headspace using SPME. After being placed in jars, samples were allowed to reach equilibrium for only $10 \mathrm{~min}$ as the ATF reported the lifetime of each sample as being approximately $4 \mathrm{hr}$. Immediately after equilibrium was reached, triplicate extractions were performed using 65um PDMS/DVB SPME fibers exposed for 30 min to extract the VOCs from each individual sample along with a blank. According to the standard compound purchased from AccuStandard®, the retention time (RT) for TATP was 11.015 minutes using the developed GC analysis method. Since the compound is not included in the NIST Mass Spectral library, the unique fragmentation pattern characteristic of the compound was used to adequately identify TATP, this pattern includes the following ions: 43, 59, 75, 101 and $117 \mathrm{~m} / \mathrm{z}$. 


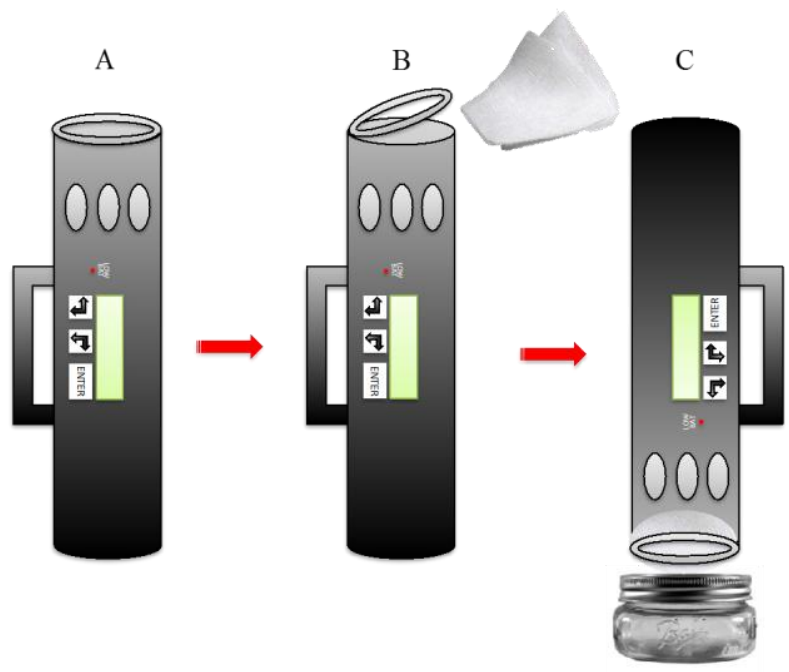

Figure 35. (A). Optimized parameters are selected using the drop down menu on the screen (B). Gauze pad is inserted and clipped in the instrument (C). The instrument is positioned upside down facing the sample for collection

GC-MS Methods

Table 25. GC-MS Methods for Target Compounds Detection

\begin{tabular}{|c|c|c|}
\hline Target Compound & SPME coating & Programming \\
\hline TATP & PDMS/DVB & $\begin{array}{l}\text { The GC injection method was } \\
\text { splitless with a constant helium flow of } \\
1 \mathrm{~mL} / \mathrm{min} \text {. The oven program was set } \\
\text { initially at } 50{ }^{\circ} \mathrm{C}(3 \mathrm{~min} \text { hold }) \text { and } \\
\text { increased to } 180{ }^{\circ} \mathrm{C} \text { at } 8.00{ }^{\circ} \mathrm{C} / \mathrm{min} \text {. The } \\
\text { inlet temperature was held constant at } \\
250{ }^{\circ} \mathrm{C} \text {. }\end{array}$ \\
\hline AN & PA & $\begin{array}{l}\text { The GC method was splitless with a } \\
\text { constant helium flow of } 1 \mathrm{~mL} / \mathrm{min} \text {. The } \\
\text { oven was initially set at } 40{ }^{\circ} \mathrm{C}(3 \mathrm{~min} \\
\text { hold), then increased to } 280{ }^{\circ} \mathrm{C}(2.5 \mathrm{~min} \\
\text { hold) at } 10{ }^{\circ} \mathrm{C} / \mathrm{min} \text {. The inlet } \\
\text { temperature was held constant at } 250{ }^{\circ} \mathrm{C} \text {. }\end{array}$ \\
\hline
\end{tabular}

* All samples were desorbed for $5 \mathrm{~min}$ 


\subsection{Results and Discussion}

Evaluation of explosives using GC-MS

\section{Ammonium Nitrate}

Ammonium nitrate is a salt thus having chemical characteristics not suitable for GCMS. was detectable. First, n-butylchloroformate was loaded on a PA fiber by sampling the headspace through a septa seal vial for 30 seconds followed by the headspace extraction of the sample for $20 \mathrm{~min}$. After the derivatization process was completed, the sample was desorbed in the GC-MS inlet for 5 min. As depicted in Figure 36, the n-butylchloroformate derivative of ammonia is obtained at 9.267 minutes and it has been confirmed directly from its mass spectrum below. 


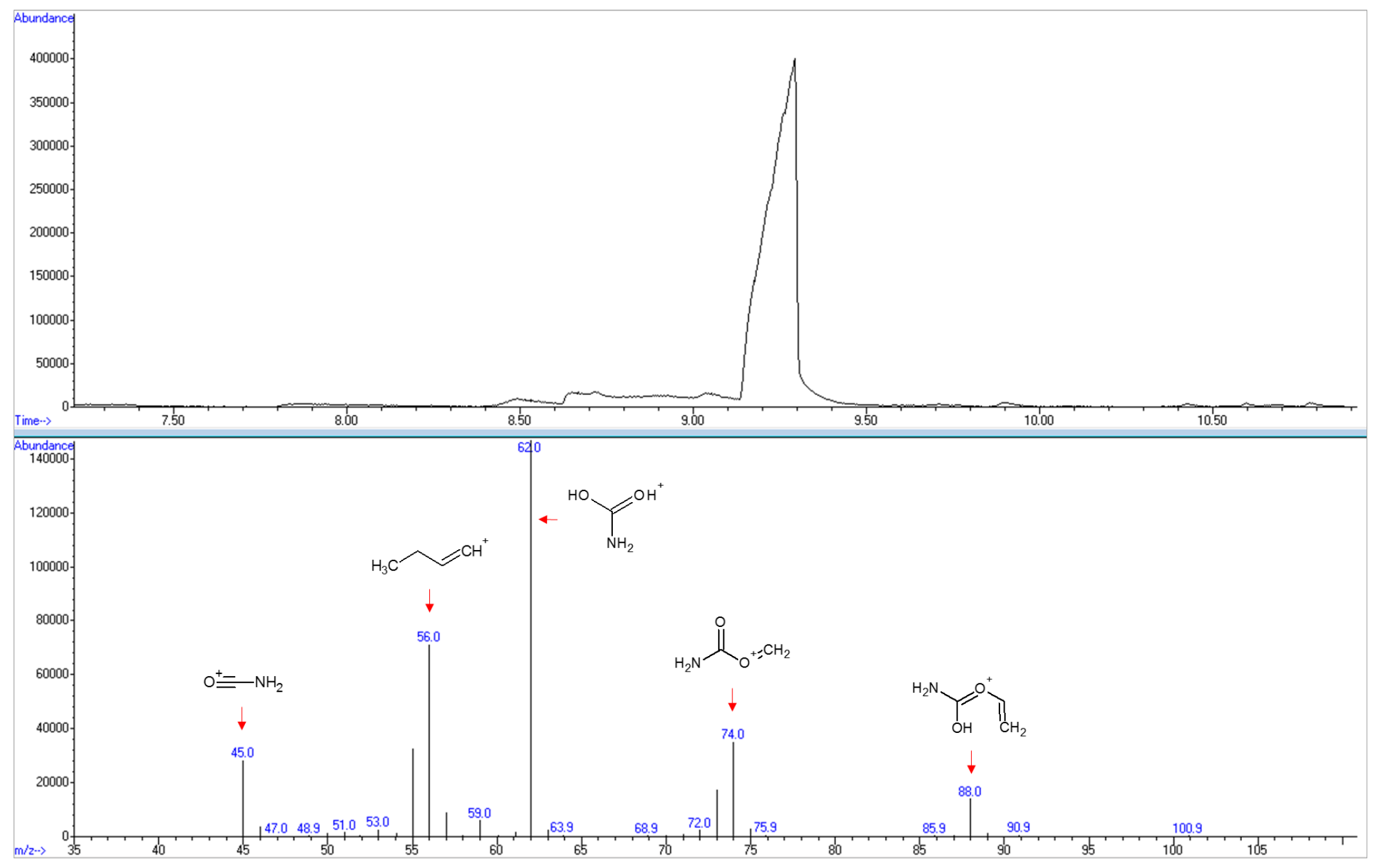

Figure 36. Ammonium Nitrate Derivatization Product Headspace Using SPME-GC-MS 


\section{Triacetone Triperoxide}

To ensure the quality and detection of TATP samples, the headspace of each was analyzed using SPME prior to its dynamic collection. According to the standard compound purchased from AccuStandard $\AA$, the retention time (RT) for TATP was $11.015 \mathrm{~min}$. Since the compound is not included in the National Institute of Standards and Technology (21) Mass Spectral library, the unique fragmentation pattern characteristic of the compound was used to adequately identify TATP, this pattern includes the following ions: 43, 59, 75, 101 and $117 \mathrm{~m} / \mathrm{z}$ (Figure 37). 


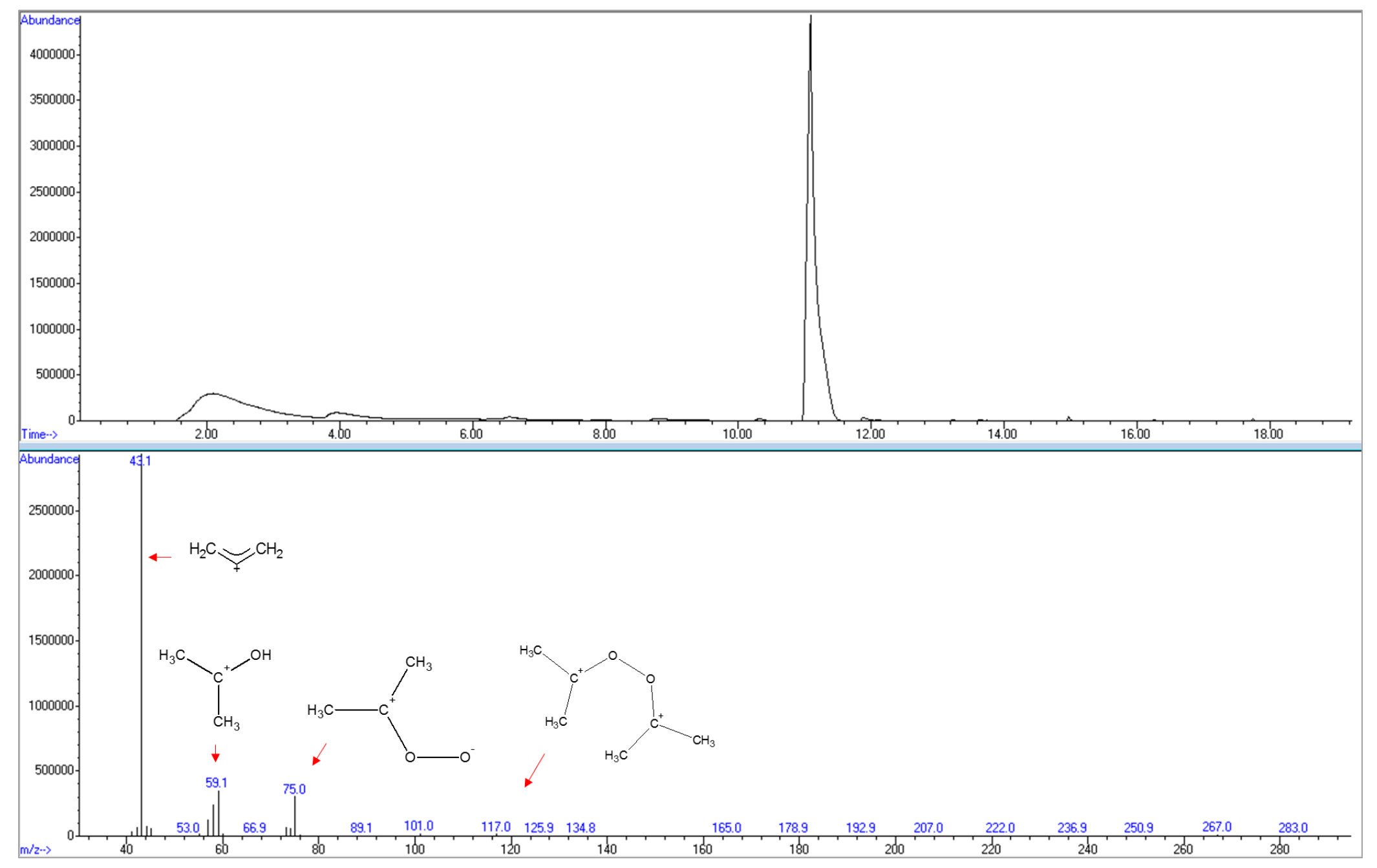

Figure 37. TATP Headspace Using SPME-GC-MS 


\section{Evaluation and Optimization of Flow Rate of the HSCS}

\section{Ammonium Nitrate}

Prior to the collection of ammonium nitrate samples preparation was required to determine the proper way of collecting the sample. A total of four different experiments were performed. Three (3) $\mathrm{mL}$ of ammonium nitrate solution was spiked onto 2"x2" cotton gauze. It was allowed 3 and 30 min to reach equilibrium. Upon completion, the VOCs were collected using different flow rates and collection times. Polyacrylate SPME fiber was exposed for $30 \mathrm{sec}$ to $10 \mu \mathrm{L}$ of n-butylchloroformate followed by the exposure of the fiber to the analyte solution for $20 \mathrm{~min}$ at room temperature and $56^{\circ} \mathrm{C}$. Subsequently the sample was desorbed in the GC-MS inlet for 5min. (Figure 38)

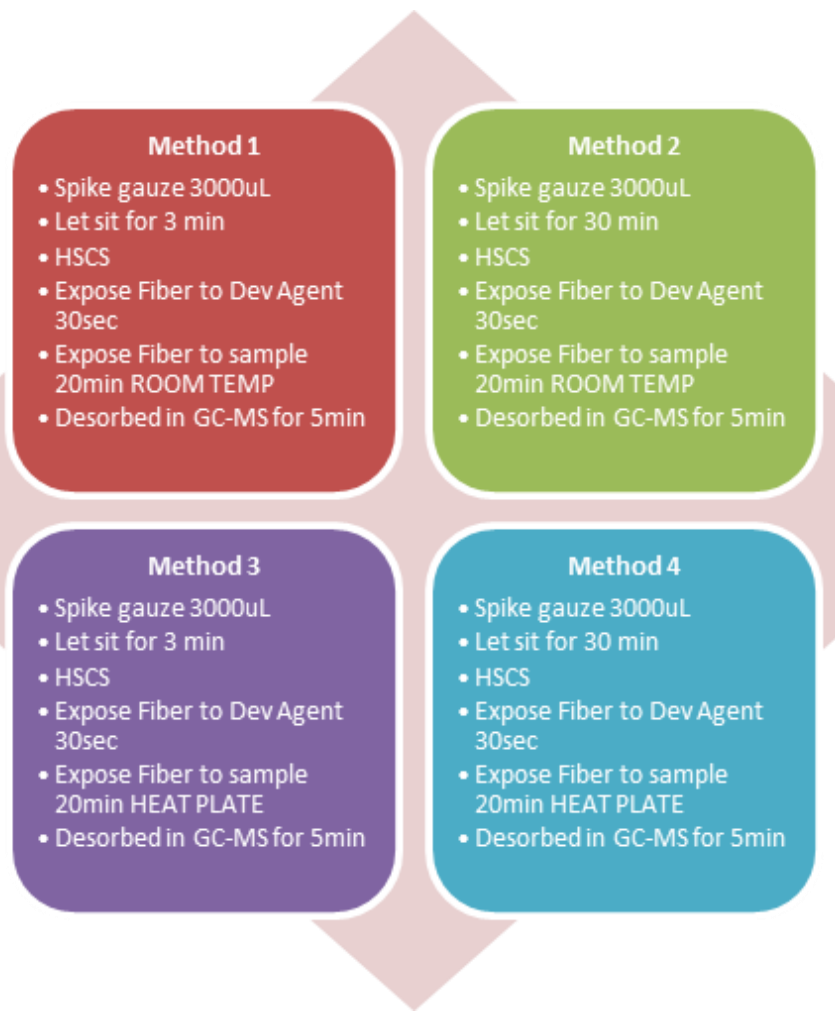

Figure 38. Equilibrium Time and Extraction Temperature Experiments for the Collection of Ammonium Nitrate 
After the analyses were performed, the data collected was analyzed. It is important to note that although the derivative product of ammonium nitrate was detected by SPME-GCMS it was not possible to be quantified. There were inconsistencies that did not allow a reliable calibration curve to be obtained, these discrepancies can be result of instrument sensitivity for this particular compound and/or breakthrough of the VOC target compound across the absorbent material. The parameters that showed promising results are summarized in Method 3 (Figure 38).

Samples were collected onto 4" x 4" cotton gauze pads, stored in $40 \mathrm{~mL}$ glass vials immediately after. As shown in

Figure 39, medium flow rate $30 \mathrm{sec}$ showed the greatest peak area (proportional to abundance). The selection was also based and supported by calculating the relative standard deviation (\%RSD), which represents the extent of variation in relation to the mean of a population. When using medium $30 \mathrm{sec}$ the least \%RSD (55.4\%) is obtained therefore, it was selected to be the optimal flow rate to be use.

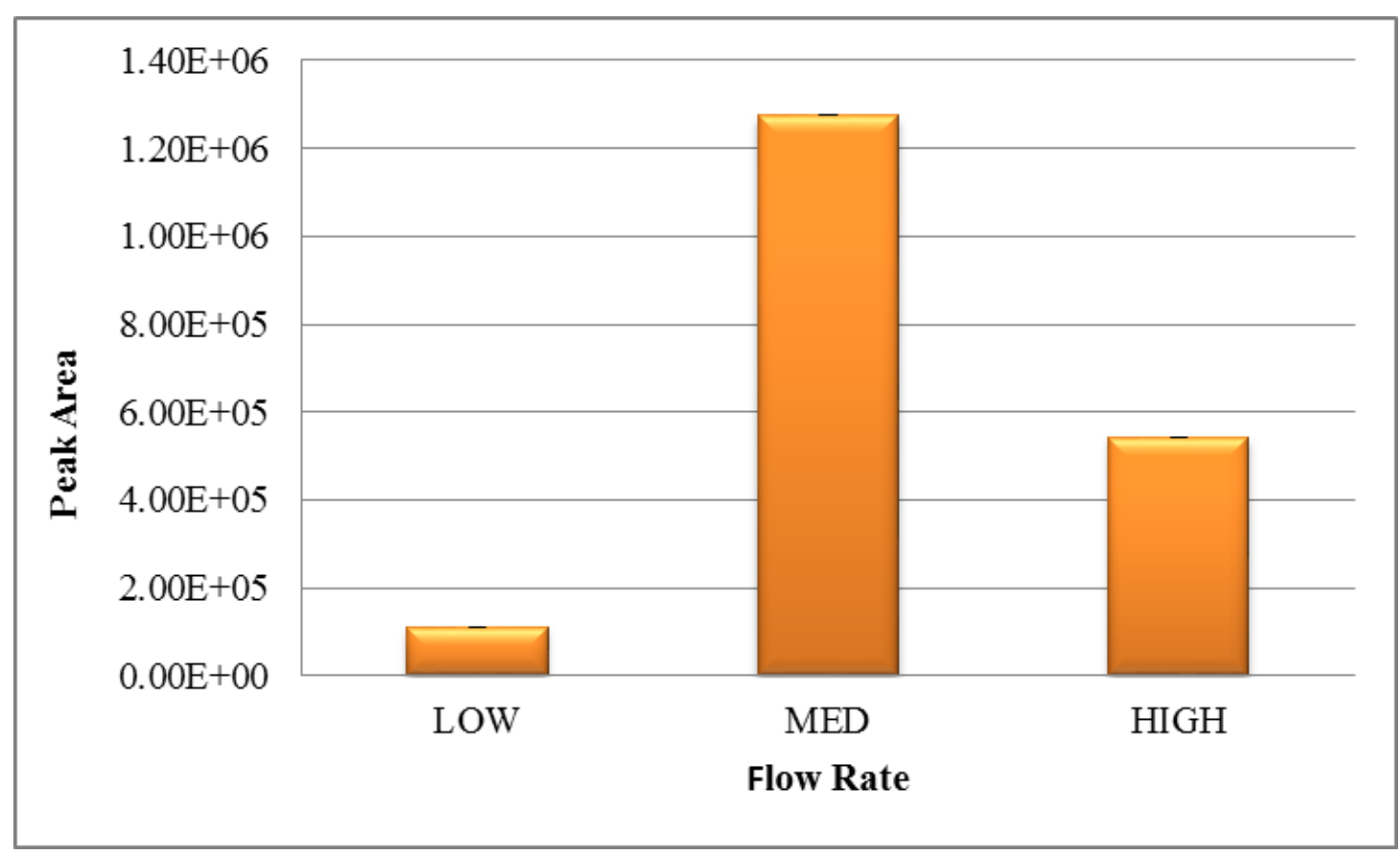

Figure 39. Flow Rate Optimization for $\mathrm{AN}$ at 30 seconds 


\section{Triacetone Triperoxide}

Triacetone triperoxide samples were collected at indoor laboratory conditions $(21.1$ ${ }^{\circ} \mathrm{C}$ with a relative humidity of $52.5 \%$ ). After the data was collected and analyzed, based on peak area (proportional to abundance), it was determined that even though low for 30 seconds was the parameter where most TATP was collected, reproducibility at this setting was poor. As a result, high for $30 \mathrm{sec}$ was considered a better flow rate for the collection of TATP. This is supported by calculating the relative standard deviation (\%RSD), which represents the extent of variation in relation to the mean of a population. As depicted in Figure 40, when samples are collected using medium $30 \mathrm{sec}$, the $\% \mathrm{RSD}$ is the greatest at $98.2 \%$. However, when high flow rate is used the \%RD is less at $21.8 \%$. The high setting was therefore considered to be the most appropriate parameter when compared with the

others. In relation to the actual amount of TATP collected, analysis of variance (ANOVA) showed no significant difference in the amounts collected using different flow rates for 30 seconds. However, the results from the high setting produced the lowest standard deviation and the most reproducible data. 


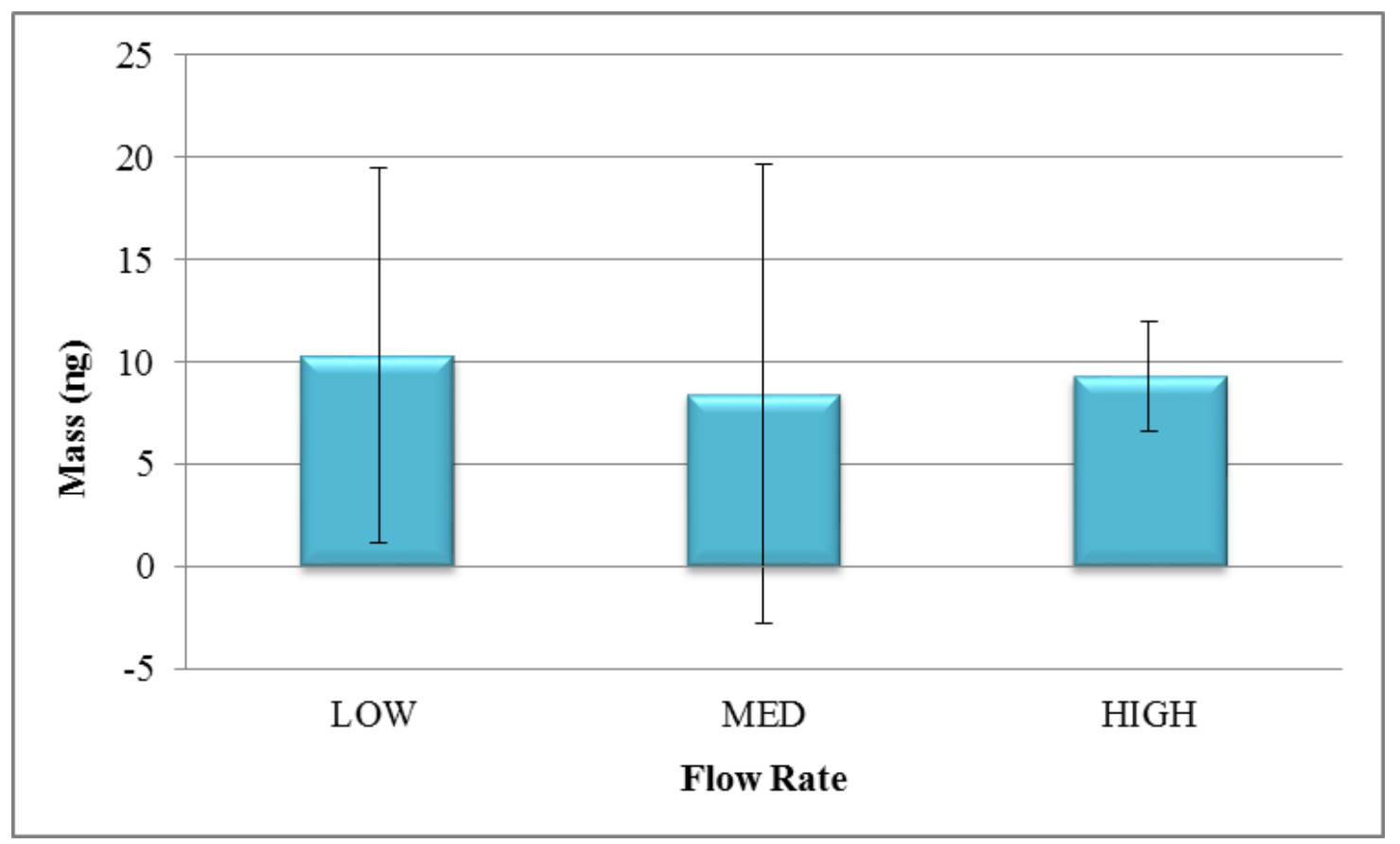

Figure 40. Flow Rate Optimization for TATP at $30 \mathrm{sec}$

Evaluation and optimization of dynamic sampling time

Ammonium Nitrate

For the optimization of the sampling time for AN, a semi-quantitative analysis using the peak area; proportional to the abundance of the target compound, was utilized for comparison purposes, using the best flow rate $30 \mathrm{sec}$ and $60 \mathrm{sec}$ collection time were compared and evaluated to determine the most suitable parameter. In order to select the best parameter for the collection of AN, the greatest peak area of the target VOC collected as well as the reproducibility of the sampling was considered.

According to the analysis on the data collected, there was significant difference between both samples shown below in Figure 41, medium for $60 \mathrm{sec}$ showed to collected more and be more reproducible than medium $30 \mathrm{sec}$. Therefore, medium $60 \mathrm{sec}$ was the 
parameter selected to be most suitable for the collection of carbamate derivative from AN in the headspace of the samples using HSCS.

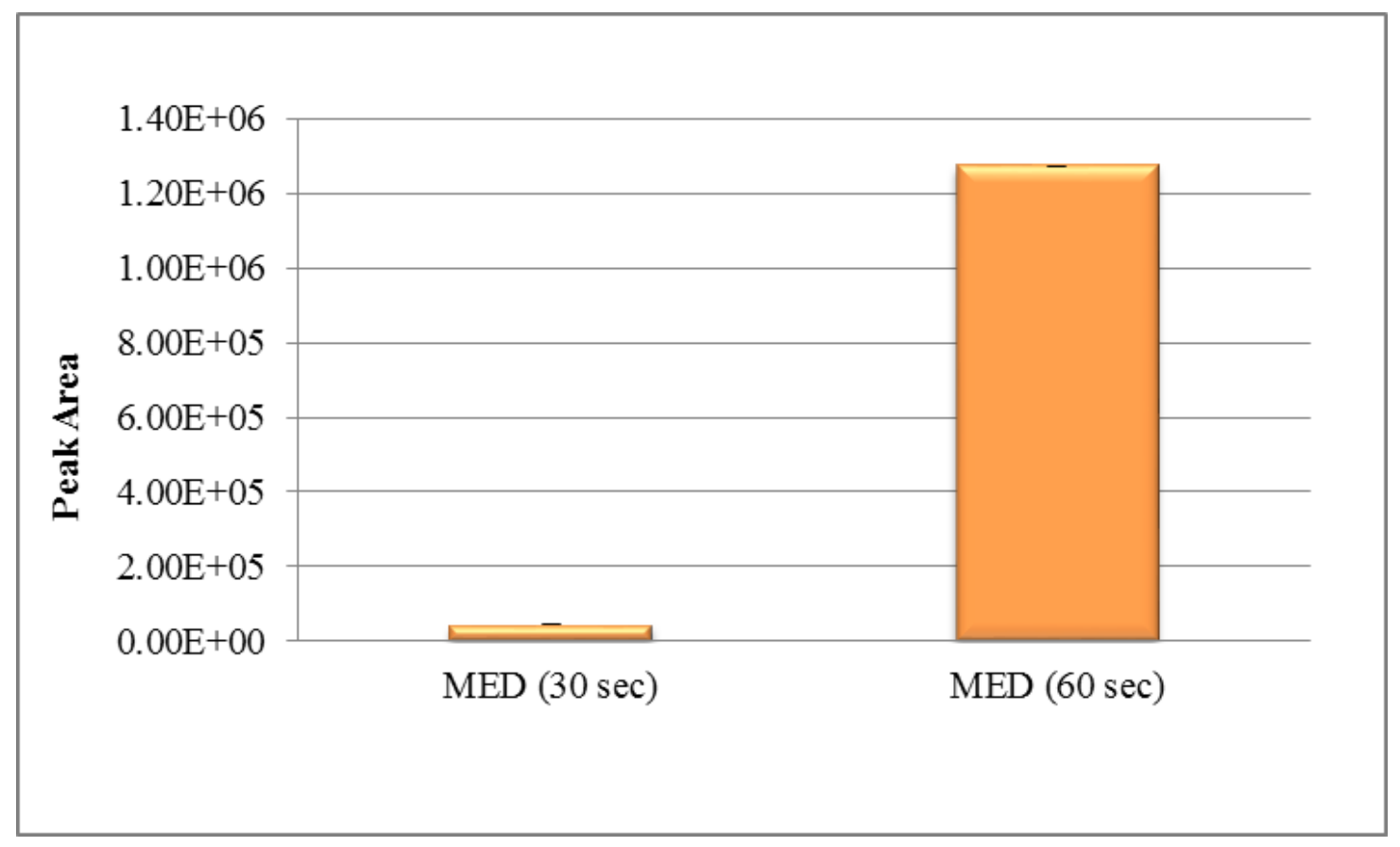

Figure 41. AN Derivative Product: Sampling Time optimization

\section{Triacetone Triperoxide}

For the optimization of the sampling time, a quantitative analysis for the collection of triacetone triperoxide (56) for $60 \mathrm{sec}$ using HSCS was also performed following the same protocol as that for the collection for $30 \mathrm{sec}$.

After the optimal flow rates were selected for each sampling time, a comparison between them was performed. In order to select the best parameter for the collection of TATP, the greatest amount of VOC collected as well as the reproducibility of the sampling was considered. Even though based on the analysis on the data collected, there was no significant difference between both samples shown below in Figure 42, high for $30 \mathrm{sec}$ showed to collect more and with higher reproducible than high collection at $60 \mathrm{sec}$. 
Therefore, high flow rate at $30 \mathrm{sec}$ was the parameter selected to be the most suitable for the collection of TATP odorant using the HSCS.

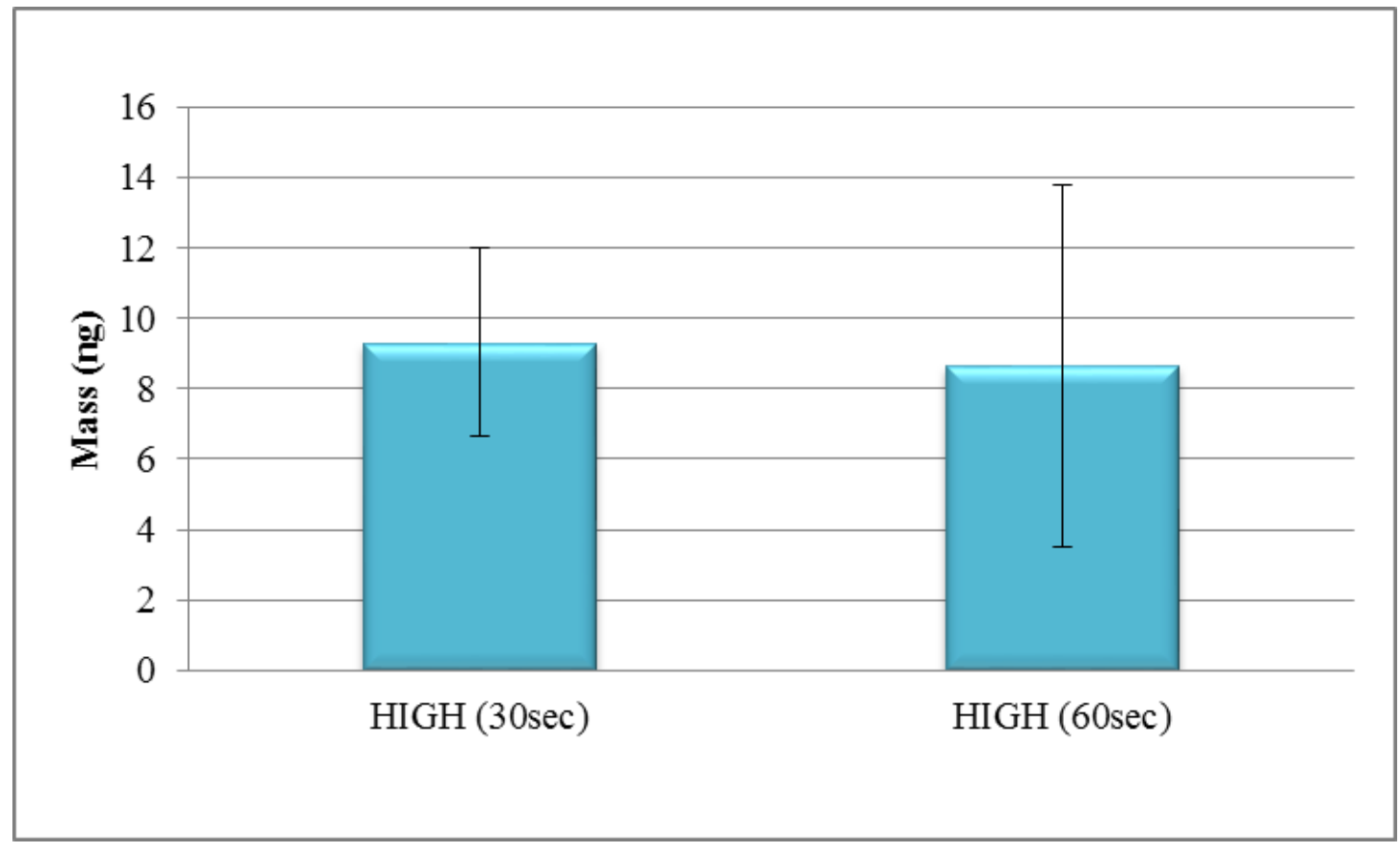

\section{Figure 42. TATP: Sampling Time Optimization}

In an effort to determine the optimal method for the collection of VOCs contained in ammonium nitrate (AN) different static collection times were analyzed. For static collection gauze pads were placed on the HSCS with the collection system powered off. The unit was setup similarly for dynamic collection but instead none of the parameters were used. The times analyzed included $0.5,1,5,15,30$ and $45 \mathrm{~min}$. Upon completion of the sample preparation, Polyacrylate (PA) SPME fiber was exposed for $30 \mathrm{sec}$ to the headspace of n-butylchloroformate $(10 \mu \mathrm{L})$ contained in a $1 \mathrm{ml}$ sealed vial followed by the exposure to the analyte solution $(1 \mathrm{mg} / \mathrm{mL})$ contained in a separate vial for $20 \mathrm{~min}$ at an average of $21.5^{\circ} \mathrm{C}$ with a $47.6 \%$ relative humidity. 
Several static collection times were analyzed for the determination of the most suitable time to be later compared to different collection methods. After the collection of triplicate samples was completed, based on the amount collected and the standard deviation among samples it was determined that the best time for the static collection of AN was 45 min (Figure 43).

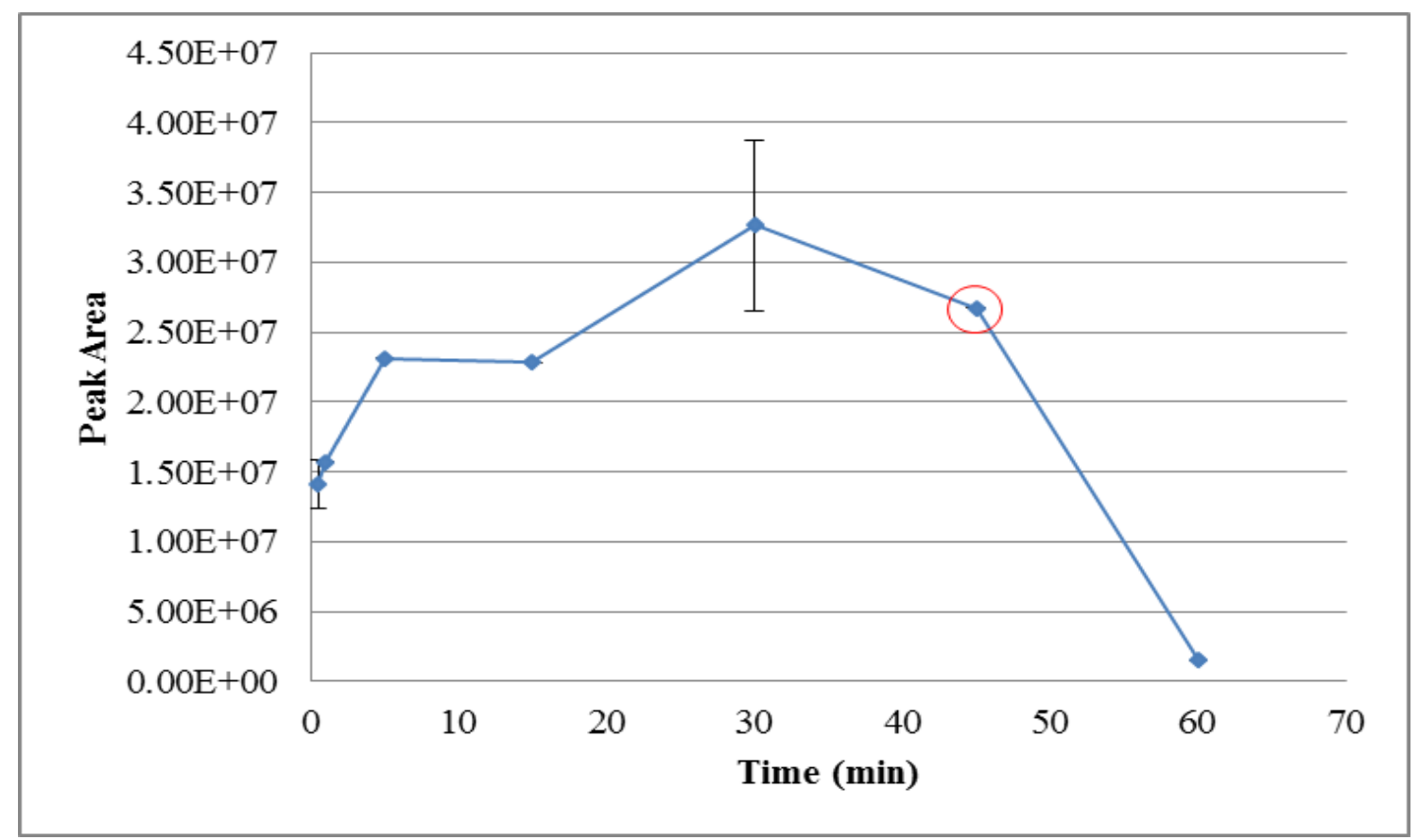

Figure 43. AN Carbamate Product: Static Collection of VOC Over Time

When the sampling time as included in the HSCS (30 and 60 seconds) is analyzed a graph as the one shown below is obtained showing that static collection provides greater collection of the target odorant (Figure 44). 


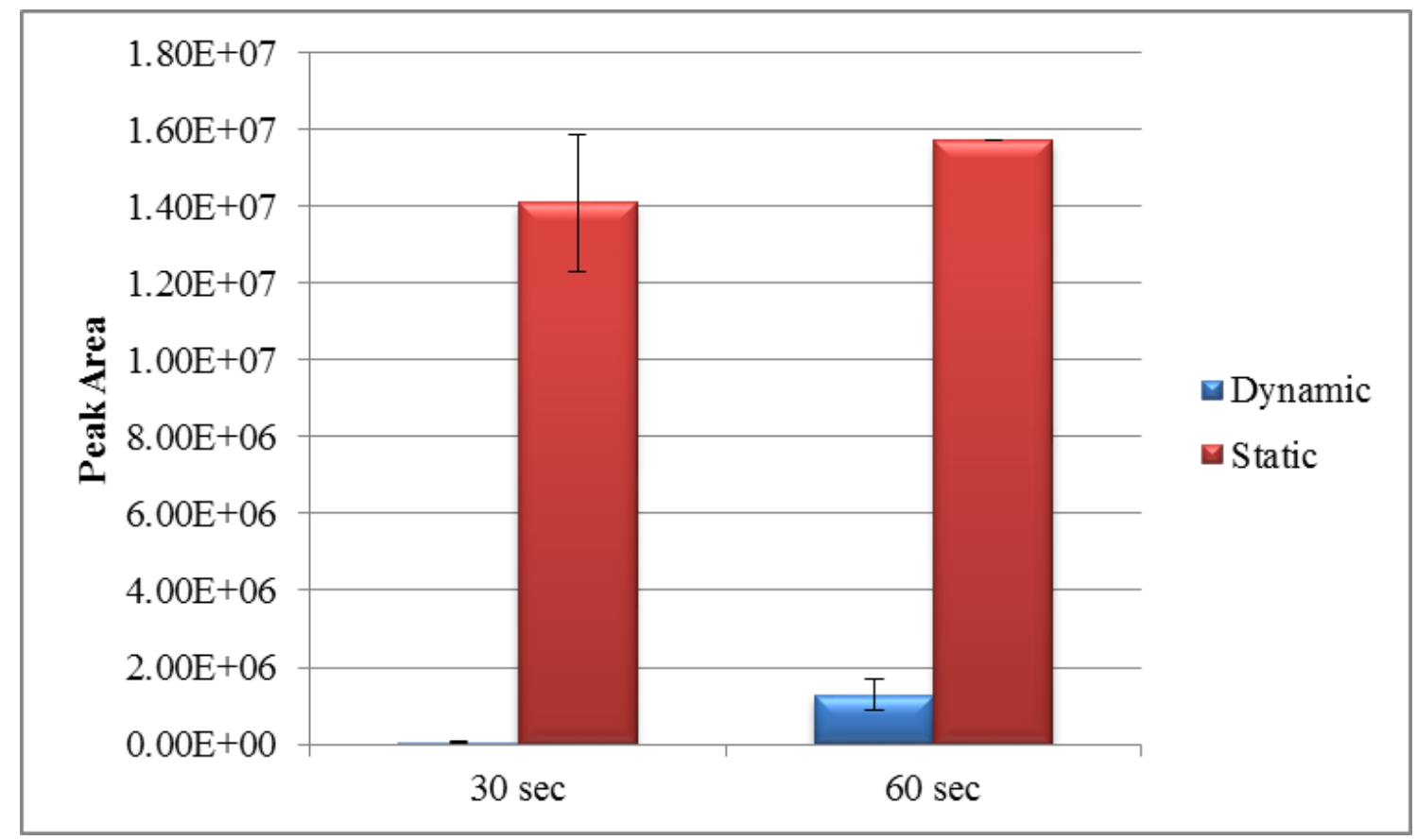

Figure 44. Static vs. Dynamic of Ammonium Nitrate Derivative

When both optimal times are analyzed and compared using different collection methods it can be seen (Figure 45) that each method is able to collect the target AN analyte, however, not in the same proportion. The purpose of this task relies on determining the ideal time for an optimal instrumental response. Therefore, it is concluded that when a gauze pad is placed in close proximity to the odor source for 45 min (static mode), the instrument shows a better response than when dynamic collection mode is performed. 


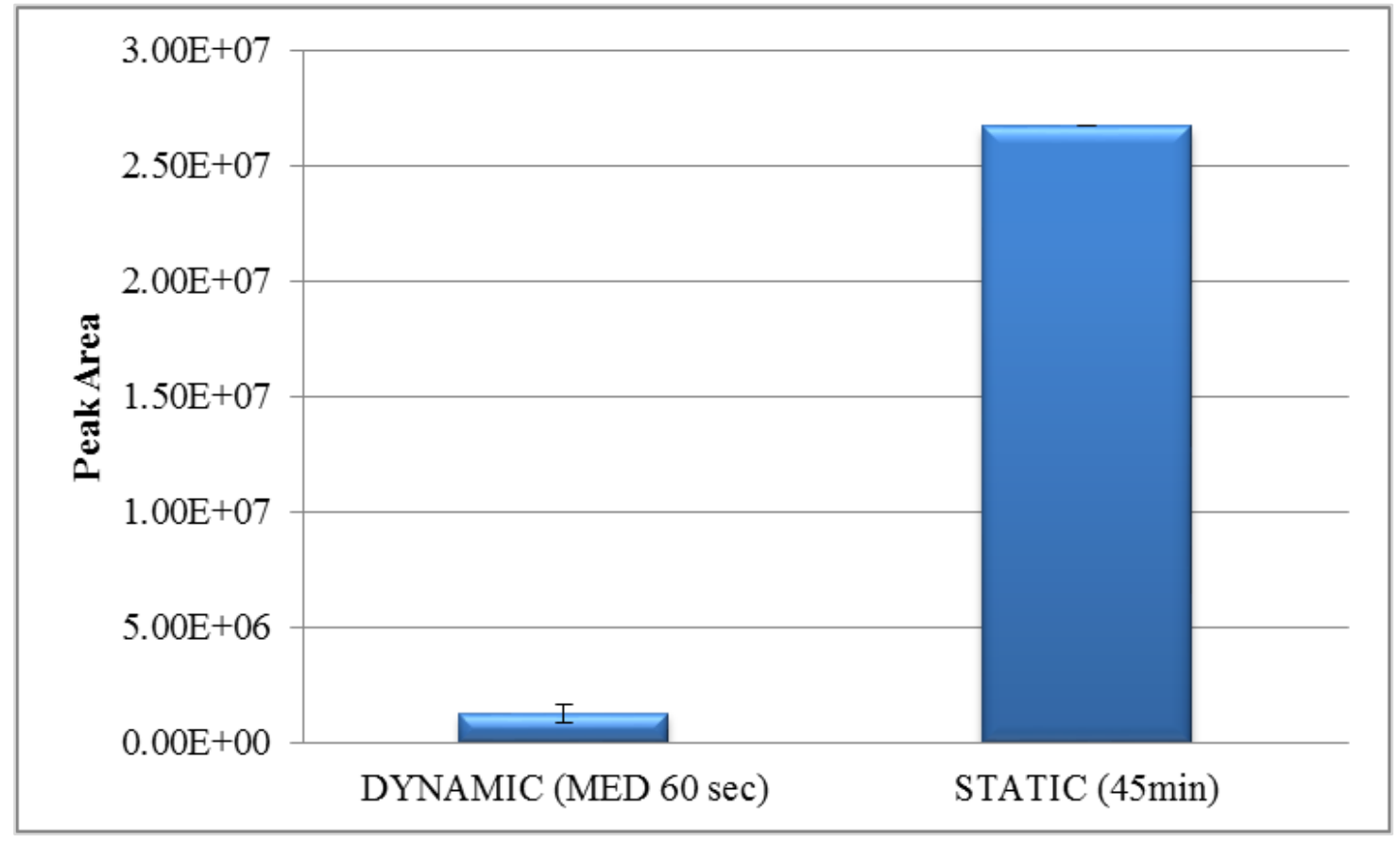

Figure 45. Comparison Between Different Dynamic and Atatic Collection Modes for Carbamate Derivative from AN

Though static collection using the dynamic collection shows greater accumulation of target VOCs, this was as a result of the extended exposure time of $45 \mathrm{~min}$ used versus the maximum time of $60 \mathrm{sec}$ used for collection in the dynamic mode. When comparing static and dynamic modes for the exact same extraction time, dynamic mode outperforms static collection by providing higher amounts of the target VOC.

\section{Field evaluation of dynamically collected VOC}

Field trial were performed, canines were presented to samples that had been aged (collected and preserved) for the specific period of time where volatiles were no longer instrumentally detected. Aged samples were collected two weeks prior to the field trial. 
Water gel was utilized as the material containing $\mathrm{AN}$ as it is an explosive material consisting of an aqueous ammonium nitrate solution. After samples were collected, they were labeled, kept in a box at ambient temperature, stored in glass vials, and sealed with parafilm to guarantee the greatest preservation of volatiles. The purpose of this task was to evaluate canines' ability to recognize the odor even though VOCs were no longer detectable through laboratory instrumentation. This phase included the search of 3 rooms out of which one included a positive control (1 lb. single based smokeless powder) and a blank room. For confirmatory purposes a canine that is certified but not participating in the trial (proofing dog) was used to run the searches areas before the trial to ensure rooms were clear of any unwanted interfering odors. Hides were placed in their designated location after the dice roll was followed and then left for at least $30 \mathrm{~min}$ prior to the beginning of the trial to guarantee the volatiles have been released. Data collected showed $50 \%$ and above combined rate of detection to water gel, while $100 \%$ combined alert rate for the positive control. Note that for the combined alert rate both alerts and interest responses have been considered (Table 26).

Table 26. Odor Recognition Test: Aged Samples $(\mathbf{n}=12)$

\begin{tabular}{ccccc}
\hline Explosive & Alert Rate (\%) & Interest Rate (\%) & $\begin{array}{c}\text { No Alert Rate } \\
(\boldsymbol{\%})\end{array}$ & $\begin{array}{c}\text { Combined Rate of } \\
\text { Detection }(\boldsymbol{\%})\end{array}$ \\
\hline Control & 100.0 & 0.0 & 0.0 & 100.0 \\
Water Gel & 25.0 & 33.3 & 41.7 & 58.3 \\
Blank & 8.3 & 8.3 & 83.4 & 16.7 \\
\hline
\end{tabular}

Fresh samples were collected the day before the trial. After collection, samples were kept in a box at ambient temperature, stored in glass vials and sealed with parafilm until 
the following day. The purpose of this task was to evaluate the performance of certified canine teams after being exposed to samples collected from a water gel using the optimal collection parameters of the collection system. The number of canines used in this trial was 11. One canine from the proficiency test was eliminated due to heat exhaustion which disabled him to complete the trial. Data collected showed $72.7 \%$ combined rate of detection for water gel while $100 \%$ for the positive control indicating canines experience.

Table 27. Odor Recognition Test: Fresh Samples (n=11)

\begin{tabular}{ccccc}
\hline Explosive & Alert Rate (\%) & Interest Rate (\%) & $\begin{array}{c}\text { No Alert Rate } \\
(\boldsymbol{\%})\end{array}$ & $\begin{array}{c}\text { Combined Rate of } \\
\text { Detection (\%) }\end{array}$ \\
\hline Control & 100.0 & 0.0 & 0.0 & 100.0 \\
Water Gel & 63.6 & 14.3 & 27.3 & 72.7 \\
Blank & 54.5 & 0.0 & 45.5 & 54.5 \\
\hline
\end{tabular}

As a complement of what has been already presented, statistical analysis was conducted to calculate the positive predictive value (PPV) and the negative predictive value $(\mathrm{NPV})$.

The PPV gives the percentage of canines that alerted, when in fact, there was an aid present. In other words, what percentage of the alerts was correct? This is calculated by dividing the true positive responses by the addition of the true positives and the false positives.

$$
P P V=\frac{\text { True Positive }}{\text { True Positive }+ \text { False Positive }}
$$


The PPV was calculated based on the canine's responses for each task. The PPV for fresh samples was $57 \%$. This means that $57 \%$ of the time the canine's alert was correct while the other $43 \%$ were false positive. However, for aged samples PPV was calculated to be $77 \%$ which corresponds for those correct alert responses while $23 \%$ of the responses are considered false positive.

On the other hand, the NPV is the percentage of canines that did not alert when there was no aid present. This percentage tells us how many of the no alert responses were correct. This is calculated by dividing the number of true negatives by the addition of true negatives and false positives.

$$
N P V=\frac{\text { True Negative }}{\text { True Negative }+ \text { False Negative }}
$$

NPV percentages were calculated based on the individual canines' response per task. For the fresh samples test the NPV was 63\% accounting for those non responses that were correct. While the percentage calculated for aged samples was $67 \%$. Negative Predictive Values should be as close to $100 \%$ as possible indicating that the canines are not alerting falsely to items that do not pose a threat. High rates of false alert for explosive detection canines are expensive as they result in unnecessary deployments of qualified bomb detection and disposal experts. The high false alert rates observed resulted from the dynamic collection of blank pads, indicating that though the dynamic collection can be effective in collecting VOCs from explosive materials, it may also be contributing substantial amounts of odor found in the atmosphere that may be interfering with canine 
responses to the blanks. Thus, extra precaution must be considered to prevent any crosscontamination (149).

\section{Transfer validation a storage containment system evaluation}

An optimization and in-depth evaluation of storage container type is an important aspect to determine optimized canine training aids for explosive detection. The dissipation of target compounds from different explosives was evaluated for up to a one month. The tendency for a compound to partition into the atmosphere is determined largely by its vapor pressure and must be considered. The vapor pressure can be regarded as a measurement of the maximum achievable amount or solubility of the substance in the vapor of air phase (169). Additionally, the collection material is of importance and must be considered when explaining the dissipation of the target compounds. A comparison of the absorbent material's performance has been previously studied by L.E. DeGreeff et al. (2011) and it has been determined that the breakthrough of compounds through the material during sampling likely plays a role in the amount of compound collected. In the same peerreviewed article, the authors evaluated different sorbent materials from which macroscopically, Dukal® gauze had the most open weave allowing compounds to breakthrough (170). The compounds that pass through the collection material (Dukal@ gauze) will therefore be considered to 'breakthrough'.

Determination of the optimal storage containment for AN was determined based on the peak area which is proportional to the amount of the compound that was present. Since the derivative product is not included in the NIST Mass Spectral library, the unique fragmentation pattern characteristic of the compound was used to adequately identify AN, this pattern includes the following ions: $44,56,62,74,88 \mathrm{~m} / \mathrm{z}$. It is important to note that 
although the derivative product of ammonium nitrate was detected by SPME-GC-MS it was difficult to accurately quantify as a result of the low abundance of volatile derivatized product detected.

L.E. DeGreeff et al. (2011) concluded that the molecular structure as well as the weave of the collection material has a major effect on the ability to trap and release volatile compounds. After a close evaluation of the data in Figure 46, it can be concluded that the trap and release capabilities of a collection material do in fact depend on its physical characteristics, but they also depend on the molecular structure of the compound(s) being collected.

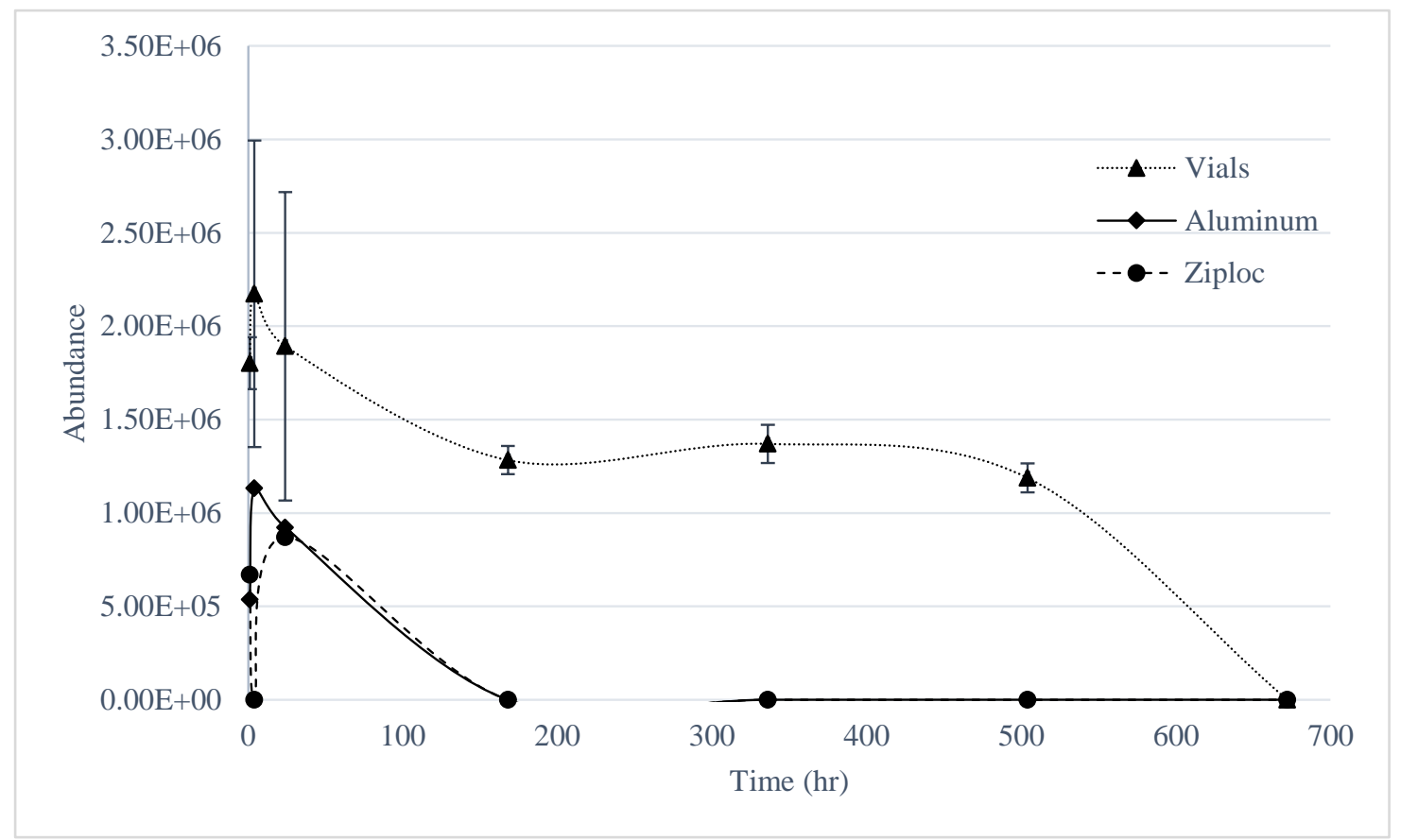

\section{Figure 46. Peresistence of Target Volatile Over Time Using Different Containment System}

Results of the persistence of ammonium nitrate over time are included in Figure 46. As can be seen, the containment that was able to hold the volatile for the longest time was 
the sealed glass vial ( 3 weeks) while, for both aluminum and ziploc bags the volatile did not last longer than one week. The inconsistency of the volatile decrease for the first 4 recorded times can be explain by its lost, since the gauze pads were being exposed to the environment for each extraction, while its increase after $4 \mathrm{hr}$ can be explained by equilibrium, since the time frame given to the volatiles to get accumulated onto the gauze pads was $24 \mathrm{hr}$ which led to greater collection of volatile and impregnation on gauze pads. However, the aluminum bag showed that during the first few hours of analysis, the volatile was only partially released into the headspace of each storage container. This is believed to be as a result of the volatile adsorbing strongly on the cotton gauze and only being partially released over time. However, as more time was allowed, the volatile was released at a greater amount which then later decreased due to dissipation into the atmosphere.

\subsection{Conclusion}

The dynamic collection of target odorants directly from explosive materials have proven to be an alternative for the newly IEDs as training aids developed using this method have shown promising results. Many commonly available materials such as gunpowder, salts, and hydrogen peroxide, can be used as explosive materials in IEDs. Common examples of explosive mixtures are ANFO and TATP both composed of a mixture of fuel and oxidizer, which fulfill the requirements to sustain a reaction as an explosive. But, concerns in regards to the transportation of these materials in a stable form have restricted daily training practices when detection teams are deployed. Additionally, the vast variety of IEDs that are developed recurrently requires a faster method to develop training aids to cover a wider range of explosives available. Therefore, this task focused on the 
optimization of the dynamic collection method of two explosive classes: salts and peroxides.

The dynamic system allows the collection of target volatiles through a suction mechanism onto a gauze pad. The system provides different parameters that are adaptable depending on the explosive family target volatile to be collected. These parameters include the flow rate and sampling time and were therefore evaluated to develop a suitable method per explosive family. As presented on Table 28 the greatest abundance for carbamate product was obtained when the VOC was collected medium for $60 \mathrm{sec}$, while TATP was most suitable collected when high for $60 \mathrm{sec}$ was used. The difference in parameters used for the collection of samples is highly dependent on the medium used to collect a target compound. Dukal® gauzes have been shown to have open weave allowing for compounds to breakthrough depending on the flow and time used for its collection (171). Additionally, Dukal ${ }^{\circledR}$ gauzes are entirely made of cotton, which has a cellular backbone containing free hydroxyl groups capable of retaining polar compounds best (172). Topological polar surface area (TPSA) of a compound is associated with the distribution of polar atoms in a molecule, carbamates have a TPSA of $66.2 \AA^{2}$, this suggests the facility to which this compound will be retained by the medium in comparison with a compounds like TATP $\left(55.4 \AA^{2}\right)(173,174)$. Furthermore, vapor pressure is directly proportional to volatility, the higher the vapor pressure, the higher the volatility. Thus, the vapor pressure is of importance as depending on its magnitude is the facility in which the compound will reach the headspace. In fact, for TATP less sampling time is enough, while AN requires more time to be collected successfully. These results supports the trend reported by Sanchez (2015) where the most volatile compounds required less time to get retained by the 
collection material nitroglycerine $(\mathrm{NG}) \approx(2,4$-dinitrotoluene) 2,4 -DNT < (2-ethyl-1hexanol) $2 \mathrm{E} 1 \mathrm{H} \approx \mathrm{DMNB}$ (2,3-dimethyl-dinitrobutane) (175).

Table 28. Suitable Parameters for the Dynamic Collection of VOCs

\begin{tabular}{ccc}
\hline Target VOC & Flow Rate & Sampling Time \\
\hline Carbamate product & Medium & $60 \mathrm{sec}$ \\
TATP & High & $30 \mathrm{sec}$ \\
\hline
\end{tabular}

From this work, it was also concluded that the dissipation of a target compound is influenced by the container in which samples are save after collection. The carbamate product from ammonium nitrate derivatization was collected for 20 days after collection which highlights the difference a container plays in sample preservation. Overall, the glass vials can retain a the target compounds for the longest time as it is the only non-permeable container, avoiding the fast release of a compound from the container, concentrating the headspace, and avoiding contamination that could potentially mask the compound in the headspace. Since this collection system approach yielded instrumental reliable results, samples certainly have the potential to be used as training aids, not only for those explosives that are currently available but also for the ever evolving improvised explosives encountered in military operations, as long as samples are well preserved.

To validate this new approach ORTs were performed. The PPV value was 57\% meaning that $57 \%$ of the time the canine's alert was correct. However, for aged samples PPV was calculated to be $77 \%$ which corresponds for those correct alert responses while $23 \%$ of the responses are considered false positive. These results highlight the potential use 
of this sort of training aids but requires different collection materials to be considered as it might improved the capacity and longevity of volatiles to be retained.

\section{CONCLUSIONS}

There are several parameters known to affect the extraction of compounds when using SPME technique for the extraction of volatiles. Therefore, the goal for the first task was to evaluate different factors that could help obtain high extraction conditions within a reasonable time. Two different SPME methods have been developed to extract VOCs from marijuana and heroin samples. Coupled with GC-MS, this SPME method showed high sensitivity and satisfactory reproducibility.

Advantages of SPME technique allows for a simple and rapid extraction, which can be later coupled to GC-MS to quantify VOCs from different illicit substances. Parameters such as fiber chemistry, extraction time, equilibrium time, and temperature were investigated an optimized to obtain the best recovery of volatiles from different illicit substances. Upon completion, differences were denoted by performing ANOVA followed by Tukey's HSD if significant difference was encountered.

Fiber chemistries varying in polarity and thickness were evaluated to identify the fiber with the best capacity for characterization of the VOCs present in the headspace of marijuana and heroin samples. The number of compounds, abundance and chemistry families extracted per fiber were considered to select the best. The $30 / 50-\mu \mathrm{m}$ DVB/CAR/PDMS fiber gave the best combined sensitivity to establish the VOC makeup of both illicit substances headspace. Different extraction times were evaluated from which 60 and 30 min for marijuana and heroin respectively, were concluded as the most suitable 
considering, number of compounds and variety of chemical families extracted at a reasonable time. Equilibrium time; the time after which the amount of analyte extracted remains constant, have also being considered in this study (12). After completing a throughout analysis, 15 and 45 min were selected as suitable time for samples of marijuana and heroin to reach equilibrium. Additionally, marijuana samples analyzed in the research were later destroyed therefore, extraction temperature was also considered as it is known to increase the partition constant of the analyte, and can be used to drive the equilibrium in favor of the gas phase. Different temperatures were evaluated, including room temperature, $30{ }^{\circ} \mathrm{C}, 40{ }^{\circ} \mathrm{C}$ and $50{ }^{\circ} \mathrm{C}$. Although increments in temperature were in fact improving the number of compounds been extracted, at higher temperatures terpenes were been comprised which lead to the selection of $40^{\circ} \mathrm{C}$.

After having successfully determined the suitable parameters for the evaluation of the volatiles in the headspace of marijuana and heroin, both substances provided by authorized personnel in South Florida were evaluated. The goal of the second task was to determine VOCs constituting the profile of these substances to later determine the volatiles responsible for the alert response of detection canines. Fibers (DVB/CAR/PDMS) were injected in triplicates to the headspace above the sample and to a blank after vials were let sit to reach equilibrium. Once the extraction was completed, one fiber was desorbed through the inlet of the GC-MS while the rest were caped to preserved the extracted volatiles. Although time and quantity were limitations in this process, SPME provided reliable results, as SPME it is a simple, fast and sensitive technique. Also, it is feasible to obtain promising results without having to alter the sample, as it is a non-destructive, not invasive extraction technique. 
As expected, the majority of the compounds found in marijuana headspace were terpenes; organic hydrocarbons produced by a wide variety of plants and that are suspected to be responsible of the scent released by the product. From the terpenes that were obtained, sesquiterpenes predominated the headspace of the dried samples. Specifically, caryophyllene, $\beta$-myrcene, linalool and limonene dominated not only in frequency of occurrence but also in abundance in the headspace giving of a pointer of what could be used as potential training aids for detection canines.

The headspace of heroin samples was also evaluated to determine those compounds that are most frequently found as well as those that are more abundant to fit the same purpose, the determination of the compounds responsible of the alert rate from detection canines. Acetic acid greatly predominated in the headspace, although acetic acid was not widely used initially when heroin was first developed, its presence can be explained by the deacetylation of acetic anhydride, the common precursor compound used for the synthesis of heroin. However, authorities started monitoring the trafficking of such precursor compound which leading the use of acetic acid instead. Although the use of acetic acid requires a much more complex synthesis, studies have shown its potential to get high yields of heroin. Finally, regardless of the type of heroin studied, acetic acid prevailed as the most abundant compound in heroin, which highlights its importance in heroin, but also the need for additional ORTs to address its potential means for the development of detector canine training aids.

The ORTs goal was to test the dog's ability to recognize some volatile odors presented for search. This practice will then led to the development of training aids that are safer to use, easy to acquire and provide consistent levels of chemicals. Compounds found in the 
headspace of both illicit substances will be used to determine the compound and/or mixture of compounds responsible for the alert response by presenting them to canines. The use of training aids is essential to maintain consistent training, which will in turn improve canines' performance and reliability. Odor recognition tests were used to determine if there was any compound(s) that stimulate an alert response when presented to the canines. Additionally, to evaluate whether or not a canine evaluated is in satisfactory working condition to perform the test, substances that they regularly trained and certified on, were included as positive controls. A satisfactory work condition was considered when canines successfully alerted to the positive control.

The use of $\operatorname{VOC}(\mathrm{s})$ as training aids has been suggested after research that have demonstrated the ability of canines to alert to a chemical associated with the drugs rather than the parent drug itself. However, no research have been able to establish anything in regards to the number of compounds or the ratio at which compounds could be use to mimic the scent of an illicit substance. Therefore, single compounds and/or mixture of compounds suspected to have great potential to be used as training aids due to their frequency in appearance or abundance in the headspace for detection canines were evaluated following SWGDOGs guideline for narcotic detection. In addition to the suspected target compound, negative control (blanks) and positive control (91) were available separately for canines to search. The ORTs revealed that there are compounds capable to elicit an alert response from detector canines. The tested compounds not only were frequently found in the headspace of the samples, but were also the most abundant. Single compounds training aids were evaluated from which limonene was the most assertive, making $100 \%$ of the canines combined alert to it. However, to have a training aid that is more representative of 
the headspace of the marijuana samples, different pool of compounds together with the use of limonene where also evaluated. As it was previously stated the best average response was obtained for mixture C (1:1 limonene: caryophyllene) with a combined alert rate of $75 \%$.

For the determination of volatiles responsible for the alert response of detection canines towards heroin, the same protocol was followed. Single-compound training aids have already been evaluated therefore, the ORTs for this particular drug was focus on the evaluation of mixture of compounds that has not been evaluated before. The mixture of compounds with the best response was for 3:1 acetic acid mixture with acetyl salicylic acid with an average response of $90 \%$. Acetic acid was frequently found in heroin headspace while acetic salicylic acid is known to be commonly used as cutting agent in the preparation of heroin.

The identification of the target odor responsible for alert response is not the only factor that needs to be considered when creating a training aid. When a training aid is been developed there are several parameters that need to taken into account for the maintenance of potency, efficacy, and functional integrity of a canine. Previous research have suggested alternatives for preservation of the training aids however, things such as the absorbent material efficiency, depends on the target chemical been used as target compound. Pseudo marijuana compounds were analyzed using gravimetric analysis. Results over time suggest a steadily dissipation over a week period when alumina is used as the absorbent material. However, when pseudo heroin was evaluated over time, the target volatile compounds stability was greatly affected by variations in humidity and temperature impact in the laboratory. Therefore, a different approach for the study of odor dissipation was 
considered. Pseudo heroin training aid was thus analyzed using ESIS (hexanal) which mechanism recompense for these inconsistencies and a more accurate data set was obtained. For this particular case both cellulose and alumina were evaluated to determine the longevity effect as response to different absorbent materials. Cellulose creates a more complex polymer chain, thus there is less space between molecules creating a better trapping source for the target compounds therefore, when compared to alumina, molecules in cellulose were retained for a longer time ( 2 weeks). It is important to note that regardless of the constitution of the pseudo training aids, when these are taken to the field, aids must not be exposed to extreme temperatures as it will have a faster permeation, but when instead training is conducted in a low temperature scenario, the dissipation of odor will reduce.

After having optimized the mimic training aids for detection canines, a field trial validation was performed to establish documented evidence that will provide a high degree of assurance of training aids quality and functionality by demonstrating canines' ability to produce the desired response consistently. Field trial validation tests were performed as ORTs to proof how feasible training aids are for detection canines. When marijuana training aid mimic, composed of a 1:1 mixture of limonene and caryophyllene, was hidden for certified detection canines to search, $66 \%$ either alerted or showed interest. Modification of the ratios, recipe and compounds that are not terpenes but may contribute to the odor released from marijuana must be considered. For validation purposes, different comestible products known to release similar terpenes as marijuana were tested to discard the statement relating canines' possibility to alert response to comestible products since some share the same compounds as the training aid would. To proof canines' discrimination power is high enough to differentiate between a single compound and a pool 
of compound containing the target chemical ORTs where performed. When canines' proficiency was evaluated, none of the canines who participated demonstrated any sort of interest on lemon peel or ground pepper both known to release terpenes.

To validate the training aid mimic for heroin, a set of ORTs varying in the location, date, and amount of training aid, were performed to demonstrate the functionality when presented to canines. Thirty grams of heroin training aid mimic separated into 3 bags containing $10 \mathrm{~g}$ each were presented to the canines for search. The idea was to simulate canine training scenarios therefore, different amounts of heroin training aid mimic were presented to the canines. Out of 12 canines. none showed difficulty in finding the aids regardless of the amount $(10,20$ or $30 \mathrm{~g})$, an average of $100 \%$ alerted to the aid as well as the positive control (heroin). Not only this demonstrates the capacity of the canines for detection but also exhibits that the combination of odorants suggested to be responsible of the alert response from canines have been satisfactory determined. Additionally, to demonstrate that the use of a target compound that is shared by commercially available product does not implicate that canines will false alert, a second set of ORTs were performed. Bayer ${ }^{\circledR}$ and distilled vinegar were tested for this purpose and no canines alerted to any of them while only one canines showed interest. This validates once again, canines' field accuracy and discrimination power is not compromised by the use of mimic training aids.

Lastly, as the use of IEDs have been the source of national security concern recently, the dynamic collection of target odorants directly from explosive materials have been considered as an alternative for development of training aids mimic. Many commercially available materials such as gunpowder, salts, and hydrogen peroxide, can be used as 
explosive materials in IEDs (e.g. ANFO and TATP). However, the instability of these materials have limited their transportation which directly affect daily training practices when detection teams are deployed. Additionally, the vast variety of IEDs that are been developed recurrently requires a faster method to develop training aids to cover a wider range of explosives available for detector canines to be familiar with. Therefore, this task focus on the optimization of the dynamic collection method of two explosive classes that have unfortunately been used in well know terrorist attacks; Oklahoma City bombing (1995) and Bombings in London (2005).

The dynamic system allows the collection of target volatiles through a vacuum mechanism onto a gauze pad. The system provides different parameters (flow rate and sampling time) that are adaptable depending on the explosive family target volatile to be collected. When the carbamate product from AN was collected the greatest abundance was obtained when the VOC was collected using medium flow rate for $60 \mathrm{sec}$, while TATP was most suitable collected when high flow rate for $60 \mathrm{sec}$ was used. The differences in parameters used for the collection of samples could be explained by the collection medium used to collect, Dukal gauzes have a cellular backbone containing free hydroxyl groups capable of retaining polar compounds best and a great open weave allowing for small molecules to breakthrough. Additionally, the vapor pressure is of importance as depending on its magnitude is the facility in which the compound will reach the headspace. In fact, for TATP less sampling time is enough, while AN requires more time to be collected successfully.

From this section, it was also concluded that the dissipation of target compounds is influenced by the type of container a sample is preserved in. Overall, the glass vials retained 
the target compounds for the longest time as it is the only non-permeable container, avoiding the fast release of the compounds from the container, concentrating the headspace of them, and avoiding contamination that could potentially mask the compounds in the headspace. This new approach yielded instrumental reliable results therefore, samples certainly have the potential to be used as training aids, not only for those explosives that are currently available but also for the ever evolving improvised explosives encountered in military operations.

Validation of this approach was performed through ORTs. Canines were presented to fresh and aged collected samples to determine the feasibility of this technique for the development of training aids for detection canines. The PPV value for the samples freshly collected was $57 \%$ meaning that $57 \%$ of the time the canine's alert was correct. However, for aged samples PPV was calculated to be $77 \%$ which also corresponds for those correct alert responses. Although these results highlight the potential use of this sort of training aids, different collection materials should be considered as a way to improve the capacity and longevity of volatiles to be retained over time. 


\section{REFERENCES}

1. United Nations Office on Drugs and Crime. Drug trafficking. , http://www.unodc.org/unodc/en/drug-trafficking/index.html (accessed on July 15, 2015).

2. Drug Enforcement Admininstration, U.S.Department of Justice. Drugs of Abuse. 2015.

3. International Drug Control Programme. Economic and Social Consequences of Drug Abuse and Illicit Trafficking. 1998. Report No.: 15.

4. Lowy, A. and McAlhany, P. Human Remain Detection with Cadaver Dogs. , http://www.crime-scene-investigator.net/cadaverdogs.html (accessed on July 15, 2016)

5. Lorenzo N, Watson SJ, Harper RJ, Hsu YL, Chow M, Rose S, Furton KG. Laboratory and field experiments used to identify Canis lupus var. familiaris active odor signature chemicals from drugs, explosives, and humans. Analytical and bioanalytical chemistry 2003;376(8):1212-24.

6. Komar D. The use of cadaver dogs in locating scattered, scavenged human remains: preliminary field test results. Journal of Forensic Sciences 1999;44(2):405-8.

7. Harper RJ, Furton KG. Biological Detection of Explosives. In: Yinon J, editor. Counterterrorist Detection Techniques of Explosives. Elsevier; 2007.

8. Furton KG, Myers LJ. The scientific foundation and efficacy of the use of canines as chemical detectors for explosives. Talanta 2001 May 10;54(3):487-500.

9. Macias MS, Harper RJ, Furton KG. A comparison of real versus simulated contraband VOCs for reliable detector dog training utilizing SPME-GC-MS. American laboratory 2008;40(1):16.

10. Pandey SK, Kim KH. Comparative analysis of odorous volatile organic compounds between direct injection and solid-phase microextraction: Development and validation of a gas chromatography mass spectrometry-based methodology. Journal of Chromatography A 2009;1216(28):5436-44. 
11. Strano-Rossi S, Chiarotti M. Solid-phase microextraction for cannabinoids analysis in hair and its possible application to other drugs. Journal of analytical toxicology 1999;23(1):7-10.

12. Pawliszyn J. Applications of solid phase microextraction. 5 ed. Royal Society of Chemistry; 1999.

13. Pawliszyn J. Handbook of Solid Phase Microextraction. Elsevier Science; 2011.

14. United States Drug Enforcement Administration. Intelligence Topics at DEA. , http://www.dea.gov/ops/intel.shtml (accessed on July 15, 2016)

15. Department of Justice U.S.Attorney's Office. Major Marijuana Trafficker Sentenced To More Than 10 Years. MIddle District of Florida. In press 2015.

16. Schoon GAA. The effect of the ageing of crime scene objects on the results of scent identification line-ups using trained dogs. Forensic science international 2005;147(1):43-7.

17. Knox L. Drugs in Ancient Culture: A History of Drug Use and Effects. Ancient Origins 2016 June 9;

18. Drug Policy Alliance. A Brief History of the Drug War., http://www.drugpolicy.org/new-solutions-drug-policy/brief-history-drug-war (accessed on November 24, 2015).

19. Musto, D. F. The History of Legislative Control Over Opium, Cocaine and Their Derivatives. , http://www.druglibrary.org/schaffer/history/ophs.htm (accessed on November 24, 2015)

20. Balster RL, Walsh S. Controlled Substances Act. Encyclopedia of Psychopharmacology. Springer; 2010. p. 354.

21. United State Drug Enforcement Administration. Drug Schedueling., http://www.dea.gov/druginfo/ds.shtml (accessed on November 10, 2015). 
22. Merriam-Webster. Narcotic. , http://www.merriamwebster.com/dictionary/narcotic (accessed on November 10, 2016).

23. Isralowitz R, Myers PL. Illicit Drugs. Greenwood; 2011.

24. Green G. The Cannabis Grow Bible: The Definitive Guide to Growing Marijuana for Recreational and Medical Use. Green Candy Press; 2003.

25. Mechoulam R, Hanus L. A historical overview of chemical research on cannabinoids. Chemistry and physics of lipids 2000;108(1):1-13.

26. Iversen LL. The science of marijuana. Oxford University Press; 2001.

27. Herkenham M, Lynn AB, Little MD, Johnson MR, Melvin LS, de Costa BR, Rice KC. Cannabinoid receptor localization in brain. Proc Natl Acad Sci U S A 1990 March;87(5):1932-6.

28. Rabinski, G. Understanding Cannabinoid Receptors: Why Cannabis Affects Humans. , https://www.whaxy.com/learn/what-are-cannabinoid-receptors (accessed on July 15, 2016).

29. National Conference of State Legislatures. State Medical Marijuana Laws. 2015 Nov 9.

30. Institute of Medicine. Marijuana and Medicine: Assessing the Science Base. National Academies Press; 1999.

31. National Center for Natural Products Research (NCNPR) Research Institute of Pharmaceutical Sciences. Quarterly Report, Potency Monitoring Project. 2010 Jan 12. Report No.: 107.

32. Substance Abuse and Mental Health Services Administration. Results from the 2013 National Survey on Drug Use and Health: Summary of National Findings Survey on Drug Use and Health: Summary of National Findings. 2014. Report No.: 14-4863.

33. Office of National Drug Control Policy. Marijuana: Know the Facts. 2010. 
34. Odell LR, Skopec J, McCluskey A. A 'cold synthesis' of heroin and implications in heroin signature analysis: Utility of trifluoroacetic/acetic anhydride in the acetylation of morphine. Forensic science international 2006;164(2):221-9.

35. Wigal D. The Mystique of Opium in History and Art. Parkstone International; 2004.

36. Fernandez H, Libby T. Heroin: Its History, Pharmacology \& Treatment. Hazelden Publishing; 2011.

37. Interview by Humberto Fernandez: Charon R, (1996).

38. National Institute on Drug Abuse. Localization of opiate binding sites within the brain and spinal cord. , https://www.drugabuse.gov/publications/teachingpackets/neurobiology-drug-addiction/section-iii-action-heroin-morphine/2localization-opiate-binding-si (accessed on July 16, 2016).

39. Rockville M. Results from the 2012 National Survey on Drug Use and Health: Summary of National Findings. Substance Abuse and Mental Health Services Administration; 2013.

40. U.S.Department of Justice. Drug Availability in the United States. ,

41. Gold MS, Eaton DH. Drugs and narcotics in history. JAMA 1996 May 1;275(17):1364-5.

42. Kelly RJ, Maghan J, Serio J. Illicit Trafficking: A Reference Handbook. ABCCLIO; 2005.

43. Lohr S. When the Money Washes Up: Offshore Banking in the Cayman Islands. The New York Times Magazine, 26-52. 3-9-1992.

44. Pastel RL, Sausa RC. Spectral differentiation of trace concentrations of NO2 from NO by laser photofragmentation with fragment ionization at 226 and $452 \mathrm{~nm}$ : quantitative analysis of NO-NO 2 mixtures. Applied optics 2000;39(15):2487-95. 
45. U.S.Department of Justice. Drug Movement Into and Within the United States. , https://www.justice.gov/archive/ndic/pubs38/38661/movement.htm (accessed on June 14, 2016)

46. Cronin M. 2015 Violence and Disruption Statistics: A dramatic scalation in hate speech, threats, and violence. 2016 Jun 2.

47. Klinger A, Klinger A. Bomb Incidents in Schools: An Analysis of 2015-2016 School Year. 2016.

48. Johnson, R. The U.S. Finally Starts Regulating Sales Of Ammonium Nitrate. , http://www.businessinsider.com/oklahoma-city-bombing-ammonium-nitratesales-regulated-2011-8 (accessed on 2 July 2016).

49. Richard, E., Windrem, R., and Connor, T. Tools of Terror: Details of Brussels Bombs Revealed. , http://www.nbcnews.com/storyline/brussels-attacks/toolsterror-details-brussels-bombs-revealed-n544141 (accessed on July 2, 2016)

50. Esposito, R., Windrem, R., and Connor, T. Tools of Terror: Details of Brussels Bombs Revealed. , 2016).

51. Akhavan J. The Chemistry of Explosives. Royal Society of Chemistry; 2011.

52. E2552 - 08. Assessing the Environmental and Human Health Impacts of New Energetic Compounds. ASTM 2014.

53. Harper RJ, Almirall JR, Furton KG. Identification of dominant odor chemicals emanating from explosives for use in developing optimal training aid combinations and mimics for canine detection. Talanta 2005 August 15;67(2):313-27.

54. Akhavan J. The Chemistry of Explosives. Cambridge, GBR: Royal Society of Chemistry; 2004.

55. Akhavan J. Classification of Explosive Materials. The Chemistry of Explosives. Royal Society of Chemistry; 2011. 
56. Oxley JC, Smith JL, Canino JN. Insensitive TATP Training Aid by Microencapsulation. Journal of Energetic Materials 2015;33(3):215-28.

57. Wolffenstein R. Ueber die Einwirkung von Wasserstoffsuperoxyd auf Aceton und Mesityloxyd. Ber Dtsch Chem Ges 1895 May 1;28(2):2265-9.

58. Liebman JF, Greer A. The Chemistry of Peroxides. Wiley; 2015.

59. Federoff BT, Sheffield OE, Kaye SM. Encyclopedia of Explosives and Related Items. New Jersey, Picatinny Arsenal.

60. Commision on Psysical Science MaA. Containing the threat from illegal bombings: An integrated national strategy for marking, tagging, rendering inert and licensing explosives and their precursors. National Academy Press, Washington DC. In press 1998.

61. Belluck P, Chang K. A NATION CHALLENGED: THE INVESTIGATION; Shoes Were a 'Homemade Bomb,' F.B.I. Agent Says. The New York Times 2001 Dec 29.

62. Mosher, D. The homemade explosive used in the Paris attacks is a chemical nightmare. , http://www.techinsider.io/paris-attack-tatp-chemical-bombs-2015-11 (accessed on August 15, 2016).

63. Mahadevan EG. Ammonium Nitrate Explosives for Civil Applications: Slurries, Emulsions and Ammonium Nitrate Fuel Oils. Wiley; 2013.

64. Akhavan J. Introduction to Explosives. The Chemistry of Explosives. Royal Society of Chemistry; 2011.

65. Ledgard J. A Soldiers Handbook, Volume 1: Explosives Operations. Jared Ledgard; 2007.

66. Horswell J. The Practice Of Crime Scene Investigation. CRC Press; 2004.

67. Zhang Z, Yang MJ, Pawliszyn J. Solid-phase microextraction. A solvent-free alternative for sample preparation. Analytical chemistry 1994;66(17):844A-53A. 
68. Wercinski SA. Solid Phase Microextraction: A PRACTICAL GUIDE. CRC Press; 1999.

69. Shirey, R. E. and Mindrup, R. F. SPME-Adsoprtion versus Absorption: Which Fiber is Best for your Application., http://www.sigmaaldrich.com/Graphics/Supelco/objects/8000/7943.pdf (accessed on March 10, 2016).

70. Smith F, Siegel JA. Handbook of Forensic Drug Analysis. Elsevier Science; 2004.

71. Smith BC. The Basics of Infrared Interpretation. Infrared Spectral Interpretation: A Systematic Approach. Taylor \& Francis; 1998.

72. Whatley H. Basic Principles and Modes of Capillary Electrophoresis. Clinical and Forensic Applications of Capillary Electrophoresis. Humana Press; 2001.

73. University of Toronto. Thin Layer ChromatographyTheory-Chemistry Online., http://www.utsc.utoronto.ca/webapps/chemistryonline/production/tlc.php (accessed on March 10, 2016).

74. Techniques CREP, Technology BCS, Studies DEL, Council NR. Existing and Potential Standoff Explosives Detection Techniques. National Academies Press; 2004.

75. Lai H, Corbin I, Almirall JR. Headspace sampling and detection of cocaine, MDMA, and marijuana via volatile markers in the presence of potential interferences by solid phase microextraction-ion mobility spectrometry (SPMEIMS). Analytical and bioanalytical chemistry 2008;392(1-2):105-13.

76. Ewing RG, Atkinson DA, Eiceman GA, Ewing GJ. A critical review of ion mobility spectrometry for the detection of explosives and explosive related compounds. Talanta 2001;54(3):515-29.

77. Fatah AA, Arcilesi RD, McClintock JA, Lattin CH, Helinski M, Hutchings M. Guide for the Selection of Explosives Detection and Blast Mitigation Equipment for Emergency First Responders. U.S. Department of Homeland Security; 2008. Report No.: 105-07. 
78. Ilias Y, Bieri S, Christen P, Veuthey JL. Evaluation of solid-phase microextraction desorption parameters for fast $\mathrm{GC}$ analysis of cocaine in coca leaves. Journal of chromatographic science 2006;44(7):394-8.

79. James AT, Martin AJP. Gas-liquid partition chromatography: the separation and micro-estimation of volatile fatty acids from formic acid to dodecanoic acid. Biochemical Journal 1952;50(5):679-90.

80. Martin AJP, Synge RM. A new form of chromatogram employing two liquid phases: A theory of chromatography. 2. Application to the micro-determination of the higher monoamino-acids in proteins. Biochemical Journal 1941;35(12):1358.

81. Rudnicka J, Buszewski B. The development of Gas Chromatography/Mass Spectrometry and Its Uses in Odor Analysis. Canine Olfaction Science and Law: Advances in Forensic Science, Medicine, Conservation, and Environmental Remediation. CRC Press; 2016.

82. Keulemans AIM. Gas chromatography. 2nd ed. New York: Reinhold Publishing Corporation; 1959.

83. Scott RPW, Perry JA. Introduction to Analytical Gas Chromatography. 2nd ed. CRC Press; 1998.

84. Bielicka-Daszkiewicz K, Voelkel A, Szejner M, Osypiuk J. Extraction properties of new polymeric sorbents in SPE/GC analysis of phenol and hydroquinone from water samples. Chemosphere 2006;62(6):890-8.

85. Sidisky L, Baney G, Stenerson K, Desorcie JL. Carrier Gas Selection for Capillary Gas Chromatography. 2011.

86. Chasteen TG. Split/Spliteless Gas Chromatography Injection. Department of Chemistry Sam Houston State University; 2000.

87. Stashenko E, Martinez JR. Gas Chromatography - Mass Spectrometry. Advances in Gas Chromatography. 2014.

88. Soderberg T. Mass Spectrometry. Organic Chemistry With a Biological Emphasis. University of Minesota; 2012. 
89. Schmidt PL. Companion animals as sentinels for public health. Veterinary Clinics of North America: Small Animal Practice 2009;39(2):241-50.

90. Verhagen R, Cox C, Machangu R, Weetjens B, Billet M. Preliminary results on the use of Cricetomys rats as indicators of buried explosives in field conditions. Mine detection dogs: training operations and odour detection Geneva: Geneva International Centre for Humanitarian Demining 2003;175-93.

91. Marshall B, Warr CG, De Bruyne M. Detection of volatile indicators of illicit substances by the olfactory receptors of Drosophila melanogaster. Chemical senses 2010;35(7):613-25.

92. Leitch O, Anderson A, Kirkbride KP, Lennard C. Biological organisms as volatile compound detectors: a review. Forensic science international 2013;232(1):92103.

93. Dogs for law enforcement. Police Canines in History., http://www.dogsforlawenforcement.org/ (accessed on June 15, 2016)

94. Furton K, Greb J, Holness H. The Scientific Working Group on Dog and Orthogonal Detector Guidelines (SWGDOG). 2010. Report No.: 231952.

95. Clay H. A treatise on the law of evidence, with a discussion of the principles and rules which govern its presentation, reception and exclusion, and the examination of witnesses in court. 2014.

96. Williams M, Johnston JM. Training and maintaining the performance of dogs (Canis familiaris) on an increasing number of odor discriminations in a controlled setting. Applied Animal Behaviour Science 2002;78(1):55-65.

97. Harvey LM, Harvey JW. Reliability of bloodhounds in criminal investigations. Journal of Forensic Sciences 2003;48(4):811-6.

98. Bird RC. Examination of the Training and Reliability of the Narcotics Detection Dog, An. Ky LJ 1996;85:405. 
99. Gazit I, Lavner Y, Bloch G, Azulai O, Goldblatt A, Terkel J. A simple system for the remote detection and analysis of sniffing in explosives detection dogs. Behavior Research Methods, Instruments, \& Computers 2003;35(1):82-9.

100. Audi T. Bones of Contention: Cadaver-sniffing canine's finds are under suspicion. Detroit Free Press. In press 2003.

101. Markon J. Bomb-Dog Trainer Sentenced For Fraud; Md. Man Supplied Government With Ineffective Canines. The Washington Post. In press 2003.

102. District Court of Appeal of Florida SD. Matheson v. State, 870 So.2d 8. 2003.

103. Beltz K. The Development of Calibrants through Characterization of Volatile Organic Compounds from Peroxide Based Explosives and a Non-target Chemical Calibration Compound 2013.

104. Johnston JM. Canine Detection Capabilities: Operational Implications of Recent R \& D Findings. 1999. Institute for Biological Detection Systems.

105. Furton KG, Harper R. Detection of Ignitable Liquid Residues in Fire Scenes Accelerant Detection Canine (ADC) Teams and other Field Tests. Advances in Forensic Science Techniques: Interpretation of Fire Scene Evidence 2004;192.

106. SWGDOG. SWGDOG SC8-Substance Detector Dogs. 2007.

107. IFRI/NFSTC. IFRI/NFSTC Certification Guidelines.

108. Papet LE. Narcotic and Explosive Odors: Volatile Organic Compounds as Training Aids for Olfactory Detection. Canine Olfaction Science and Law: Advances in Forensic Science, Medicine, Conservation, and Environmental Remediation. CRC Press; 2016.

109. Strobel RRN, Noll R, Kury J. Nitromethane K-9 Detection Limit. 2001.

110. Kranz WD, Strange NA, Goodpaster JV. Fooling fido-Chemical and behavioral studies of pseudo-explosive canine training aids. Analytical and bioanalytical chemistry 2014;406(30):7817-25. 
111. Kranz W, Kitts K, Strange N, Cummins J, Lotspeich E, Goodpaster J. On the smell of Composition C-4. Forensic science international 2014;236:157-63.

112. Furton KG, Harper RJ. Controlled Odor Mimic Permeation System. 7-18-2007. Google Patents.

113. Yinon J. Counterterrorist Detection Techniques of Explosives. Elsevier Science; 2011.

114. Harper RJ. Improving the scientific reliability of biological detection of explosives by Canis Familiaris: Active odour signatures and their implications 2005.

115. Neuhaus W. Uber die Riechscharfe des Hundes fur Fettsauren. Journal of Comparative Physiology A: Neuroethology, Sensory, Neural, and Behavioral Physiology 1953;35(6):527-52.

116. Syrotuck WG. Anatomy and Physiology. Scent and the Scenting Dog. Barkleigh Productions Incorporated; 2000.

117. Lawson MJ, Craven BA, Paterson EG, Settles GS. A computational study of odorant transport and deposition in the canine nasal cavity: implications for olfaction. Chemical senses 2012;37(6):553-66.

118. Bainbridge D. The Anatomy of the Canine Nose. Canine Olfaction Science and Law: Advances in Forensic Science, Medicine, Conservation, and Environmental Remediation. CRC Press; 2016.

119. Schoon A, Haak R. How do dogs smell? Odor Perception. K9 Suspect Discrimination: Training and Practicing Scent Identification Line-Ups. Brush Education; 2002.

120. Quignon P, Galibert F. Genetics of Canine Olfaction. Canine Olfaction Science and Law: Advances in Forensic Science, Medicine, Conservation, and Environmental Remediation. CRC Press; 2016. 
121. Settles GS. Sniffers: fluid-dynamic sampling for olfactory trace detection in nature and homeland securityГÇöthe 2004 freeman scholar lecture. Journal of Fluids Engineering 2005;127(2):189-218.

122. Fisher RA. Statistical methods for research workers. Genesis Publishing Pvt Ltd; 1925.

123. Roberts M, Russo R. A Student's Guide to Analysis of Variance. Taylor \& Francis; 2014.

124. Statistics D. Statistical Primer for Cardiovascular Research. Circulation 2006;114:76-81.

125. Mayer D. Essential Evidence-Based Medicine. Cambridge University Press; 2004.

126. DeGreeff LE, Weakley-Jones B, Furton KG. Creation of training aids for human remains detection canines utilizing a non-contact, dynamic airflow volatile concentration technique. Forensic science international 2012;217(1):32-8.

127. Ledingham KWD. The use of lasers to detect strategic and environmentally sensitive materials. Physica Scripta 1995;1995(T58):100.

128. Parmeter JE. The challenge of standoff explosives detection.: IEEE; 2004 p. 3558.

129. Robert Shirey. Selecting the Appropriate SPME Fiber Coating -Effect of Analyte Molecular Weight and Polarity. 2015.

130. Hood LVS, Dames ME, Barry GT. Headspace volatiles of marijuana. 1973.

131. PerkinElmer, Inc. An Introduction to Headspace Sampling in Gas Chromatography., http://www.perkinelmer.com/PDFs/downloads/GDE_Intro_to_Headspace.pdf (accessed on July 13, 2015). 
132. Rice S. Investigating the aroma of marijuana, cocaine, and heroin for forensic applications using simultaneous multidimensional gas chromatography-mass spectrometry-olfactometry Iowa State University; 2015.

133. Heidari HR, Shahtaheri SJ, Golbabaei F, Alimohammadi M, Rahimi-Froushani A. Optimization of headspace solid phase microextraction procedure for trace analysis of toluene. International Journal of Occupational Safety and Ergonomics 2008;14(4):395-405.

134. Vu DT. SPME/GC-MS characterization of volatiles associated with methamphetamine: Toward the development of a pseudomethamphetamine training material. Journal of Forensic Science 2001;46(5):1014-24.

135. Hoffman EM, Curran AM, Dulgerian N, Stockham RA, Eckenrode BA. Characterization of the volatile organic compounds present in the headspace of decomposing human remains. Forensic science international 2009;186(1):6-13.

136. Hood LVS, Barry GT. Headspace volatiles of marihuana and hashish: gas chromatographic analysis of samples of different geographic origin. Journal of Chromatography A 1978;166(2):499-506.

137. Larroque V, Desauziers V, Mocho P. Study of preservation of polydimethylsiloxane/Carboxen solid-phase microextraction fibres before and after sampling of volatile organic compounds in indoor air. Journal of Chromatography A 2006;1124(1):106-11.

138. Green DE. Highly sensitive adaptable procedures for mass spectrometric real-time monitoring of drugs and other compounds. Intra Sci Chem Rep 1970;4:211-21.

139. Cadola L, Broseus J, Esseiva P. Chemical profiling of different hashish seizures by gas chromatography-mass spectrometry and statistical methodology: A case report. Forensic science international 2013;232(1):e24-e27.

140. Osman A, Thorpe JW, Caddy B. Comparison of Cannabis Samples from Different Origins by the Headspace Technique and an Assessment of Chromatographic Traces Using the r-Matrix. Journal of the Forensic Science Society 1985;25(6):427-33. 
141. Farooqi AHA, Samgwan NS, Sangwan RS. Effect of different photoperiodic regimes on growth, flowering and essential oil in Mentha species. Plant Growth Regulation 1999;29(3):181-7.

142. Osman A, Caddy B. Analysis of cannabis using Tenax-GC. Journal of the Forensic Science Society 1985;25(5):377-84.

143. Da Porto C, Decorti D, Natolino A. Ultrasound-assisted extraction of volatile compounds from industrial Cannabis sativa L. inflorescences. International Journal of Applied Research in Natural Products 2014;7(1):8-14.

144. Rather MA, Dar BA, Sofi SN, Tauheeda H, Nasir A, Lone AH. Headspace solid phase microextraction (HS SPME) gas chromatography mass spectrometric analysis of the volatile constituents of Cannabis sativa L. from Kashmir. J Pharm Res 2011;4(8):2651-3.

145. Graedel TE. Terpenoids in the atmosphere. Reviews of Geophysics 1979;17(5):937-47.

146. Goggin C. Characterizing the Odor of Marijuana using Direct Analysis in Real Time Mass Spectrometry. UNIVERSITY OF CALIFORNIA, DAVIS; 2013.

147. Showbotham L. Brief of Amici Curiae, Fourth Amendment Scholars in Support of Respondent, Florida v. Jardines. Loy L Rev 2012;58:1101.

148. Dudareva N, Murfitt LM, Mann CJ, Gorenstein N, Kolosova N, Kish CM, Bonham C, Wood K. Developmental regulation of methyl benzoate biosynthesis and emission in snapdragon flowers. The Plant Cell 2000;12(6):949-61.

149. Cerreta MM, Furton KG. An assessment of detection canine alerts using flowers that release methyl benzoate, the cocaine odorant, and an evaluation of their behavior in terms of the VOCs produced. Forensic science international 2015;251:107-14.

150. Chiarotti M, Fucci N. Analysis of volatile compounds in heroin samples. Forensic science international 1988;37(1):47-53. 
151. Ciccarone D. Heroin in brown, black and white: Structural factors and medical consequences in the US heroin market. International Journal of Drug Policy 2009;20(3):277-82.

152. Flanary DH. Search, Seizure, and Dogs "Attacking K-9 Evidence". 2012.

153. Jezierski T, Ensminger J, Papet LE. Narcotic and Explosive Odors: Volatile Organic Compounds as Training Aids for Olfactory Detection. Canine Olfaction Science and Law: Advances in Forensic Science, Medicine, Conservation, and Environmental Remediation. CRC Press; 2016.

154. Macias MS. The development of an optimized system of narcotic and explosive contraband mimics for calibration and training of biological detectors 2009.

155. United Nations Publications. World Drug Report 2007. 2007.

156. Harper RJ, Almirall JR, Furton KG. Identification of dominant odor chemicals emanating from explosives for use in developing optimal training aid combinations and mimics for canine detection. Talanta 2005;67(2):313-27.

157. Furton KG, Harper RJ, Perr JM, Almirall JR. Optimization of biological and instrumental detection of explosives and ignitable liquid residues including canines, SPME/ITMS and GC/MSn.: International Society for Optics and Photonics; 2003 p. 183-92.

158. Shah VP, Midha KK, Findlay JW, Hill HM, Hulse JD, McGilveray IJ, McKay G, Miller KJ, Patnaik RN, Powell ML. Bioanalytical method validation - a revisit with a decade of progress. Pharmaceutical research 2000;17(12):1551-7.

159. Sigma Aldrich Co. Safety Data Sheet.

160. Lueck E. Acetic acid. Antimicrobial food additives, characteristics, uses, effects. Springer; 1980. p. 167-74.

161. Scientific America. Why do flowers have scent?, www.scientificamerican.com/article/why-do-flowers-have-scent (accessed on July 18, 2016) 
162. Moore DS. Instrumentation for trace detection of high explosives. Review of Scientific Instruments 2004;75(8):2499-512.

163. Fjellanger R, Andersen EK, McLean I. A training program for filter-search mine detection dogs. International Journal of Comparative Psychology 2002;15(4).

164. Lotspeich E, Kitts K, Goodpaster J. Headspace concentrations of explosive vapors in containers designed for canine testing and training: theory, experiment, and canine trials. Forensic science international 2012;220(1):130-4.

165. Hikal WM, Weeks BL. Determination of sublimation rate of 2, 4, 6trinitrotoluene (TNT) nano thin films using UV-absorbance spectroscopy. Journal of thermal analysis and calorimetry 2011;110(2):955-60.

166. Stefanuto PH, Perrault KA, Stadler S, Pesesse R, LeBlanc HN, Forbes SL, Focant JF. GC GC-TOFMS and supervised multivariate approaches to study human cadaveric decomposition olfactive signatures. Analytical and bioanalytical chemistry 2015;407(16):4767-78.

167. Stadler S, Stefanuto PH, Byer JD, Brokl M, Forbes S, Focant JF. Analysis of synthetic canine training aids by comprehensive two-dimensional gas chromatography-time of flight mass spectrometry. Journal of Chromatography A 2012;1255:202-6.

168. Tipple CA, Caldwell PT, Kile BM, Beussman DJ, Rushing B, Mitchell NJ, Whitchurch CJ, Grime M, Stockham R, Eckenrode BA. Comprehensive characterization of commercially available canine training aids. Forensic science international 2014;242:242-54.

169. Dionne BC, Rounbehler DP, Achter EK, Hobbs JR, Fine DH. Vapor pressure of explosives. Journal of Energetic Materials 1986;4(1-4):447-72.

170. Curran AM. The analytical determination of the uniqueness and persistence of the volatile components of human scent using optimized collection methods Florida International University; 2005. 
171. DeGreeff LE, Curran AM, Furton KG. Evaluation of selected sorbent materials for the collection of volatile organic compounds related to human scent using non-contact sampling mode. Forensic science international 2011;209(1):133-42.

172. DeGreeff LE, Furton KG. Collection and identification of human remains volatiles by non-contact, dynamic airflow sampling and SPME-GC/MS using various sorbent materials. Analytical and bioanalytical chemistry 2011;401(4):1295-307.

173. Pubchem. Carbamate. Pubchem.ncbi.nlm.nih.gov (accessed on September 24, 2016)

174. Pubchem. Triacetone Triperoxide. Pubchem.ncbi.nlm.nih.gov (accessed on September 24, 2016)

175. Sanchez CL. Evaluation of the Scent Collection System for Its Effectiveness in Volatile Organic Compound Collection and Use in Canine Training 2015. 


\section{APPENDICES}

Table 29. Chemical Product Physical and Chemical Properties

\begin{tabular}{|c|c|c|c|c|c|}
\hline Compound & CAS Number & $\begin{array}{l}\text { Molecular } \\
\text { Formula } \\
\end{array}$ & $\begin{array}{l}\text { Formula Weight } \\
(\mathrm{g} / \mathrm{mol})\end{array}$ & $\begin{array}{l}\text { Vapor Pressure } \\
25^{\circ} \mathrm{C}(\mathrm{mmHg})\end{array}$ & $\%$ Purity \\
\hline$\alpha$-pinene & $80-56-8$ & $\mathrm{C}_{10} \mathrm{H}_{16}$ & 136.24 & 4.75 & 98 \\
\hline$\beta$-pinene & $18172-67-3$ & $\mathrm{C}_{10} \mathrm{H}_{16}$ & 136.24 & 2.39 & 99 \\
\hline 3-carene & $13466-78-9$ & $\mathrm{C}_{10} \mathrm{H}_{16}$ & 136.24 & 3.72 & 90 \\
\hline Limonene & $5989-27-5$ & $\mathrm{C}_{10} \mathrm{H}_{16}$ & 136.24 & 1.98 & 97 \\
\hline Linalool & $78-70-6$ & $\mathrm{C}_{10} \mathrm{H}_{18} \mathrm{O}$ & 154.25 & 0.016 & 97 \\
\hline Fenchyl alcohol & $1632-73-1$ & $\mathrm{C}_{10} \mathrm{H}_{18} \mathrm{O}$ & 154.25 & 0.069 & 96 \\
\hline Borneol & $464-45-9$ & $\mathrm{C}_{10} \mathrm{H}_{18} \mathrm{O}$ & 154.25 & 0.040 & 99 \\
\hline$\beta$-caryophellene & $87-44-5$ & $\mathrm{C}_{15} \mathrm{H}_{24}$ & 204.35 & 0.013 & $80+$ \\
\hline Alloaromadendrene & $25246-27-9$ & $\mathrm{C}_{15} \mathrm{H}_{24}$ & 204.35 & 0.023 & 98 \\
\hline Caryophellene oxide & $1139-30-6$ & $\mathrm{C}_{15} \mathrm{H}_{24} \mathrm{O}$ & 220.35 & 0.007 & 95 \\
\hline
\end{tabular}


Table 30. Canine rooster and trials schedule (Marijuana)

\begin{tabular}{|c|c|c|c|}
\hline FIU Number & Canine Name & Date & Location \\
\hline 12 & Nina & $2 / 2 / 2015$ & Kendall Warehouse \\
\hline 21 & Sting & $2 / 2 / 2015$ & Kendall Warehouse \\
\hline 13 & Thor & $2 / 2 / 2015$ & Kendall Warehouse \\
\hline 16 & Ricco & $2 / 3 / 2015$ & Homestead Warehouse \\
\hline 22 & Jake & $2 / 3 / 2015$ & Homestead Warehouse \\
\hline 23 & Milo & $2 / 3 / 2015$ & Homestead Warehouse \\
\hline 24 & Apache & $2 / 3 / 2015$ & Homestead Warehouse \\
\hline 21 & Sting & $2 / 3 / 2015$ & Homestead Warehouse \\
\hline 12 & Nina & $2 / 3 / 2015$ & Homestead Warehouse \\
\hline 26 & Chucky & $2 / 25 / 2015$ & Miami Dade Corrections \\
\hline 25 & Shadow & $2 / 25 / 2015$ & Miami Dade Corrections \\
\hline 22 & Jake & $3 / 25 / 2015$ & Schoolboard Warehouse \\
\hline 12 & Nina & $3 / 25 / 2015$ & Schoolboard Warehouse \\
\hline 24 & Apache & $3 / 25 / 2015$ & Schoolboard Warehouse \\
\hline 21 & Sting & $3 / 25 / 2015$ & Schoolboard Warehouse \\
\hline 27 & Loki & $3 / 25 / 2015$ & Schoolboard Warehouse \\
\hline 16 & Ricco & $10 / 7 / 2015$ & Homestead Warehouse \\
\hline 30 & Shadow & $10 / 7 / 2015$ & Homestead Warehouse \\
\hline 24 & Apache & $10 / 7 / 2015$ & Homestead Warehouse \\
\hline 31 & Tango & $10 / 7 / 2015$ & Homestead Warehouse \\
\hline 22 & Jake & $10 / 7 / 2015$ & Homestead Warehouse \\
\hline 16 & Ricco & $11 / 4 / 2015$ & Schoolboard Warehouse \\
\hline
\end{tabular}


Schoolboard Warehouse

23

Milo

$11 / 4 / 2015$

Tango

$11 / 4 / 2015$

Schoolboard Warehouse

31

31

Tango

$3 / 16 / 2016$

Schoolboard Warehouse

30

Shadow

$3 / 16 / 2016$

Schoolboard Warehouse 
Table 31. Canine ORTs Single Compound Raw Data (Marijuana n=27)

\begin{tabular}{|c|c|c|c|c|c|c|}
\hline Canine & Limonene & $\alpha$-Pinene & Caryophyllene & $\beta$-Pinene & Linalool & $\begin{array}{c}\text { Pos. } \\
\text { Control }\end{array}$ \\
\hline 12 & A & A & $\mathrm{N}$ & I & I & A \\
\hline 12 & $\mathrm{I}$ & $\mathrm{N}$ & $\mathrm{N}$ & A & A & A \\
\hline 12 & $\mathrm{~N}$ & A & $\mathrm{N}$ & A & $\mathrm{N}$ & A \\
\hline 13 & $\mathrm{~A}$ & A & $\mathrm{N}$ & A & $\mathrm{N}$ & $\mathrm{A}$ \\
\hline 16 & A & A & A & A & A & A \\
\hline 16 & A & A & A & - & A & A \\
\hline 16 & $\mathrm{~N}$ & A & A & - & $\mathrm{A}$ & A \\
\hline 21 & A & A & $\mathrm{N}$ & A & $\mathrm{N}$ & A \\
\hline 21 & I & I & $\mathrm{N}$ & A & A & A \\
\hline 21 & A & A & A & $\mathrm{N}$ & $\mathrm{N}$ & A \\
\hline 22 & A & $\mathrm{N}$ & A & A & A & A \\
\hline 22 & $\mathrm{I}$ & $\mathrm{N}$ & A & $\mathrm{N}$ & $\mathrm{N}$ & A \\
\hline 22 & A & A & I & - & $\mathrm{N}$ & A \\
\hline 23 & $\mathrm{I}$ & A & $\mathrm{I}$ & I & A & A \\
\hline 23 & $\mathrm{I}$ & $\mathrm{I}$ & $\mathrm{I}$ & - & $\mathrm{N}$ & A \\
\hline 24 & A & $\mathrm{I}$ & $\mathrm{I}$ & $\mathrm{I}$ & A & A \\
\hline 24 & I & $\mathrm{N}$ & I & $\mathrm{N}$ & $\mathrm{N}$ & $\mathrm{A}$ \\
\hline 24 & A & $\mathrm{N}$ & $\mathrm{N}$ & $\mathrm{N}$ & $\mathrm{N}$ & A \\
\hline 25 & $\mathrm{~N}$ & $\mathrm{~N}$ & I & $\mathrm{N}$ & $\mathrm{N}$ & A \\
\hline 26 & $\mathrm{~N}$ & $\mathrm{~N}$ & $\mathrm{~N}$ & $\mathrm{~N}$ & $\mathrm{~N}$ & $\mathrm{~A}$ \\
\hline 27 & I & $\mathrm{N}$ & I & $\mathrm{N}$ & $\mathrm{N}$ & A \\
\hline 30 & I & $\mathrm{N}$ & I & - & $\mathrm{I}$ & $\mathrm{A}$ \\
\hline 30 & I & I & $\mathrm{N}$ & - & I & A \\
\hline 30 & $\mathrm{~N}$ & $\mathrm{~N}$ & $\mathrm{~N}$ & $\mathrm{~N}$ & $\mathrm{~N}$ & $\mathrm{~A}$ \\
\hline 31 & I & $\mathrm{N}$ & $\mathrm{A}$ & - & $\mathrm{I}$ & $\mathrm{A}$ \\
\hline 31 & $\mathrm{I}$ & $\mathrm{N}$ & I & - & $\mathrm{N}$ & A \\
\hline 31 & A & $\mathrm{N}$ & $\mathrm{N}$ & $\mathrm{N}$ & I & A \\
\hline
\end{tabular}


Table 32. Canine rooster and trials schedule (Heroin)

\begin{tabular}{lllll}
\hline FIU Number & Canine Name & Handler & Date & Location \\
\hline 12 & Nina & J. Cardenas & $2 / 10 / 2014$ & Kendall Warehouse \\
13 & Thor & V. Hernandez & $2 / 11 / 2014$ & Kendall Warehouse \\
& & & & \\
16 & Ricco & Russ & $9 / 11 / 2014$ & Homestead Warehouse \\
22 & & & & \\
12 & Jake & J. Cuellar & $3 / 25 / 2014$ & Schoolboard Warehouse \\
24 & Nina & J. Cardenas & $3 / 25 / 2014$ & Schoolboard Warehouse \\
21 & Apache & G. Hernandez & $3 / 25 / 2014$ & Schoolboard Warehouse \\
27 & Sting & B. Swikehardt & $3 / 25 / 2014$ & Schoolboard Warehouse \\
& Loki & A. Sandoval & $3 / 25 / 2014$ & Schoolboard Warehouse \\
21 & & & & \\
13 & Sting & B. Swikehardt & $9 / 9 / 2015$ & Westwood Christian School \\
29 & Thor & V. Hernandez & $9 / 9 / 2015$ & Westwood Christian School \\
30 & Kobee & C. Riddick & $9 / 9 / 2015$ & Westwood Christian School \\
24 & Shadow & D. Cates & $9 / 9 / 2015$ & Westwood Christian School \\
27 & Apache & G. Hernandez & $9 / 9 / 2015$ & Westwood Christian School \\
31 & Loki & A. Sandoval & $9 / 9 / 2015$ & Westwood Christian School \\
22 & Tango & C. Ferland & $9 / 9 / 2015$ & Westwood Christian School \\
12 & Jake & J. Cuellar & $9 / 9 / 2015$ & Westwood Christian School \\
31 & Nina & J. Cardenas & $9 / 9 / 2015$ & Westwood Christian School \\
29 & Robee & C. Riddick & $9 / 20 / 2015$ & Kendall Park Warehouse II \\
& Shadow & D. Cates & $9 / 20 / 2015$ & Kendall Park Warehouse II \\
& Loki & A. Sandoval & $9 / 20 / 2015$ & Kendall Park Warehouse II \\
& & G. Hernandez & $9 / 20 / 2015$ & Kendall Park Warehouse II \\
& Russ & $9 / 20 / 2015$ & Kendall Park Warehouse II
\end{tabular}




$\begin{array}{lllll}13 & \text { Thor } & \text { V. Hernandez } & 9 / 20 / 2015 & \text { Kendall Park Warehouse II } \\ 22 & \text { Jake } & \text { J. Cuellar } & 9 / 20 / 2015 & \text { Kendall Park Warehouse II } \\ 29 & \text { Kobee } & \text { C. Riddick } & 10 / 21 / 2015 & \text { Kendall Warehouse } \\ 16 & \text { Ricco } & \text { E. Russ } & 10 / 21 / 2015 & \text { Kendall Warehouse } \\ 30 & \text { Shadow } & \text { D. Cates } & 10 / 21 / 2015 & \text { Kendall Warehouse } \\ 31 & \text { Tango } & \text { C. Ferland } & 10 / 21 / 2015 & \text { Kendall Warehouse } \\ 21 & & & & \\ & \text { Sting } & \text { B. Swikehardt } & 1 / 13 / 2016 & \text { Miami Dade Public School }\end{array}$


Table 33. Canine ORTs Single Compound Raw Data (Heroin n=30)

\begin{tabular}{|c|c|c|c|c|c|}
\hline Canine & BZA & SA & Blank & ASA & PC \\
\hline 12 & A & A & $\mathrm{N}$ & A & A \\
\hline 13 & I & A & $\mathrm{N}$ & A & A \\
\hline 16 & - & - & $\mathrm{N}$ & $\mathrm{A}$ & A \\
\hline 22 & $\mathrm{~N}$ & A & A & I & A \\
\hline 12 & $\mathrm{~N}$ & A & $\mathrm{N}$ & A & A \\
\hline 24 & $\mathrm{~N}$ & $\mathrm{~N}$ & $\mathrm{~N}$ & I & A \\
\hline 21 & A & A & $\mathrm{N}$ & A & A \\
\hline 27 & $\mathrm{~N}$ & $\mathrm{~N}$ & $\mathrm{~N}$ & I & A \\
\hline 21 & A & A & $\mathrm{N}$ & A & A \\
\hline 13 & A & A & $\mathrm{N}$ & A & A \\
\hline 29 & $\mathrm{~N}$ & I & $\mathrm{N}$ & $\mathrm{N}$ & A \\
\hline 30 & I & I & $\mathrm{N}$ & $\mathrm{N}$ & A \\
\hline 24 & A & A & $\mathrm{N}$ & A & A \\
\hline 27 & $\mathrm{~N}$ & $\mathrm{~N}$ & $\mathrm{~N}$ & I & A \\
\hline 31 & A & $\mathrm{N}$ & $\mathrm{N}$ & $\mathrm{N}$ & A \\
\hline 22 & A & A & $\mathrm{N}$ & A & A \\
\hline 12 & A & A & $\mathrm{N}$ & A & A \\
\hline 31 & A & A & $\mathrm{N}$ & A & A \\
\hline 29 & A & I & $\mathrm{N}$ & I & A \\
\hline 30 & I & I & $\mathrm{N}$ & I & A \\
\hline 27 & $\mathrm{~N}$ & I & $\mathrm{N}$ & I & A \\
\hline 24 & $\mathrm{~N}$ & I & $\mathrm{N}$ & I & A \\
\hline 16 & A & A & $\mathrm{N}$ & A & A \\
\hline 13 & $\mathrm{~N}$ & A & $\mathrm{N}$ & A & A \\
\hline 22 & A & A & $\mathrm{N}$ & A & A \\
\hline 29 & $\mathrm{~N}$ & A & $\mathrm{N}$ & A & A \\
\hline 16 & A & A & $\mathrm{N}$ & A & A \\
\hline 30 & $\mathrm{~N}$ & $\mathrm{~N}$ & $\mathrm{~N}$ & I & A \\
\hline 31 & $\mathrm{~N}$ & $\mathrm{~N}$ & $\mathrm{~N}$ & A & A \\
\hline 21 & I & $\mathrm{N}$ & $\mathrm{N}$ & A & A \\
\hline
\end{tabular}


Table 34. Canine rooster and trials schedule (Pseudo Heroin)

\begin{tabular}{|c|c|c|c|c|}
\hline Canine & Canine & Handler & Date & Location \\
\hline 12 & Nina & J. Cardenas & 02/10/16 & FIU Tech Warehouse \\
\hline 19 & Boss & I. Perez & $02 / 10 / 16$ & FIU Tech Warehouse \\
\hline 29 & Kobee & C. Riddick & $02 / 10 / 16$ & FIU Tech Warehouse \\
\hline 31 & Tango & C. Ferland & $02 / 10 / 16$ & FIU Tech Warehouse \\
\hline 22 & Jake & J. Cuellar & $02 / 10 / 16$ & FIU Tech Warehouse \\
\hline 30 & Shadow & D. Cates & $02 / 10 / 16$ & FIU Tech Warehouse \\
\hline 27 & Loki & A. Sandoval & $02 / 10 / 16$ & FIU Tech Warehouse \\
\hline 21 & Sting & B. Swikehardt & $02 / 10 / 16$ & FIU Tech Warehouse \\
\hline 13 & Thor & V. Hernandez & $02 / 10 / 16$ & FIU Tech Warehouse \\
\hline 31 & Tango & C. Ferland & $02 / 17 / 16$ & Miami Dade School Warehouse \\
\hline 13 & Thor & V. Hernandez & $02 / 17 / 16$ & Miami Dade School Warehouse \\
\hline 30 & Shadow & D. Cates & $02 / 17 / 16$ & Miami Dade School Warehouse \\
\hline 22 & Jake & J. Cuellar & 02/17/16 & Miami Dade School Warehouse \\
\hline 21 & Sting & B. Swikehardt & $02 / 17 / 16$ & Miami Dade School Warehouse \\
\hline 31 & Tango & C. Ferland & 03/09/16 & FIU Vehicles \\
\hline 22 & Jake & J.Cuellar & 03/09/16 & FIU Vehicles \\
\hline 29 & Kobee & C. Riddick & 03/09/16 & FIU Vehicles \\
\hline 34 & & & $03 / 30 / 16$ & Miami Dade School Warehouse \\
\hline 30 & Shadow & D. Cates & 03/30/16 & Miami Dade School Warehouse \\
\hline 29 & Kobee & C. Riddick & 03/30/16 & Miami Dade School Warehouse \\
\hline 31 & Tango & C. Ferland & 03/30/16 & Miami Dade School Warehouse \\
\hline 22 & Jake & J. Cuellar & 03/30/16 & Miami Dade School Warehouse \\
\hline 24 & Apache & G. Hernandez & 03/30/16 & Miami Dade School Warehouse \\
\hline
\end{tabular}




\begin{tabular}{lllll}
12 & Nina & J. Cardenas & $04 / 13 / 16$ & Miami Dade School Warehouse \\
29 & Kobee & C. Riddick & $04 / 13 / 16$ & Miami Dade School Warehouse \\
35 & & & $04 / 13 / 16$ & Miami Dade School Warehouse \\
31 & Tango & C. Ferland & $04 / 13 / 16$ & Miami Dade School Warehouse \\
22 & Jake & J. Cuellas & $04 / 13 / 16$ & Miami Dade School Warehouse \\
13 & Thor & V. Hernadez & $04 / 13 / 16$ & Miami Dade School Warehouse \\
\hline
\end{tabular}


Table 35. Canine ORTs Pseudo Heroin Raw Data $(n=12)$

\begin{tabular}{cccccccc}
\hline Canine No. & 20 & 20 & 10 & 30 & 30 & 20 & 30 \\
\hline 12 & A & A & A & A & - & - & A \\
19 & A & A & - & - & - & - & - \\
29 & A & A & - & - & A & A & A \\
31 & A & A & - & - & A & A & A \\
22 & A & A & A & A & A & A & A \\
30 & A & A & A & A & A & - & - \\
27 & A & A & - & - & - & - & - \\
21 & A & A & A & A & - & - & - \\
13 & A & A & A & A & - & - & A \\
34 & - & - & - & - & - & A & - \\
24 & - & - & - & - & - & A & - \\
35 & - & - & - & - & - & - & A \\
\hline
\end{tabular}




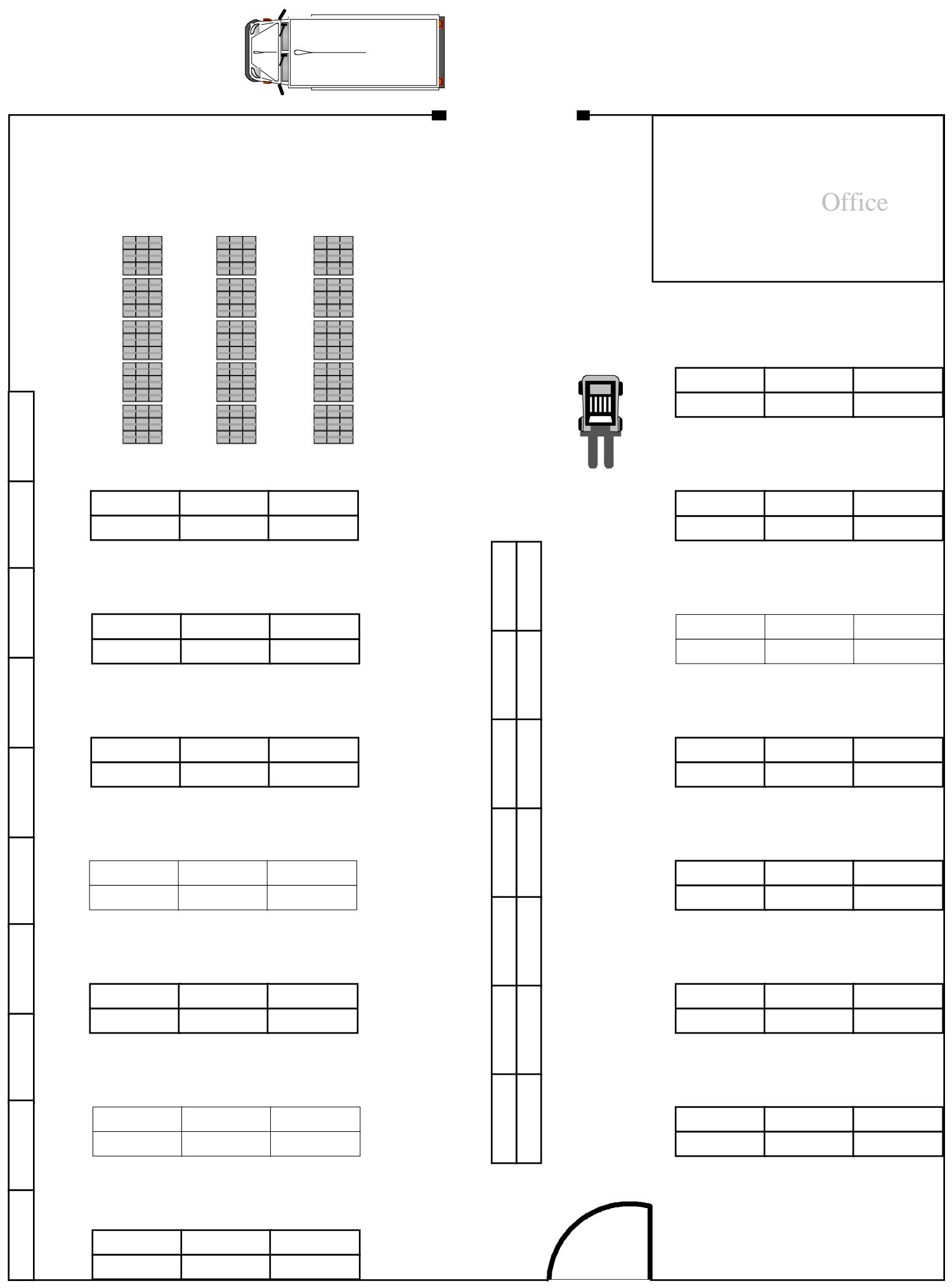

Figure 47. Kendall Park Warehouse 8000 ft2 


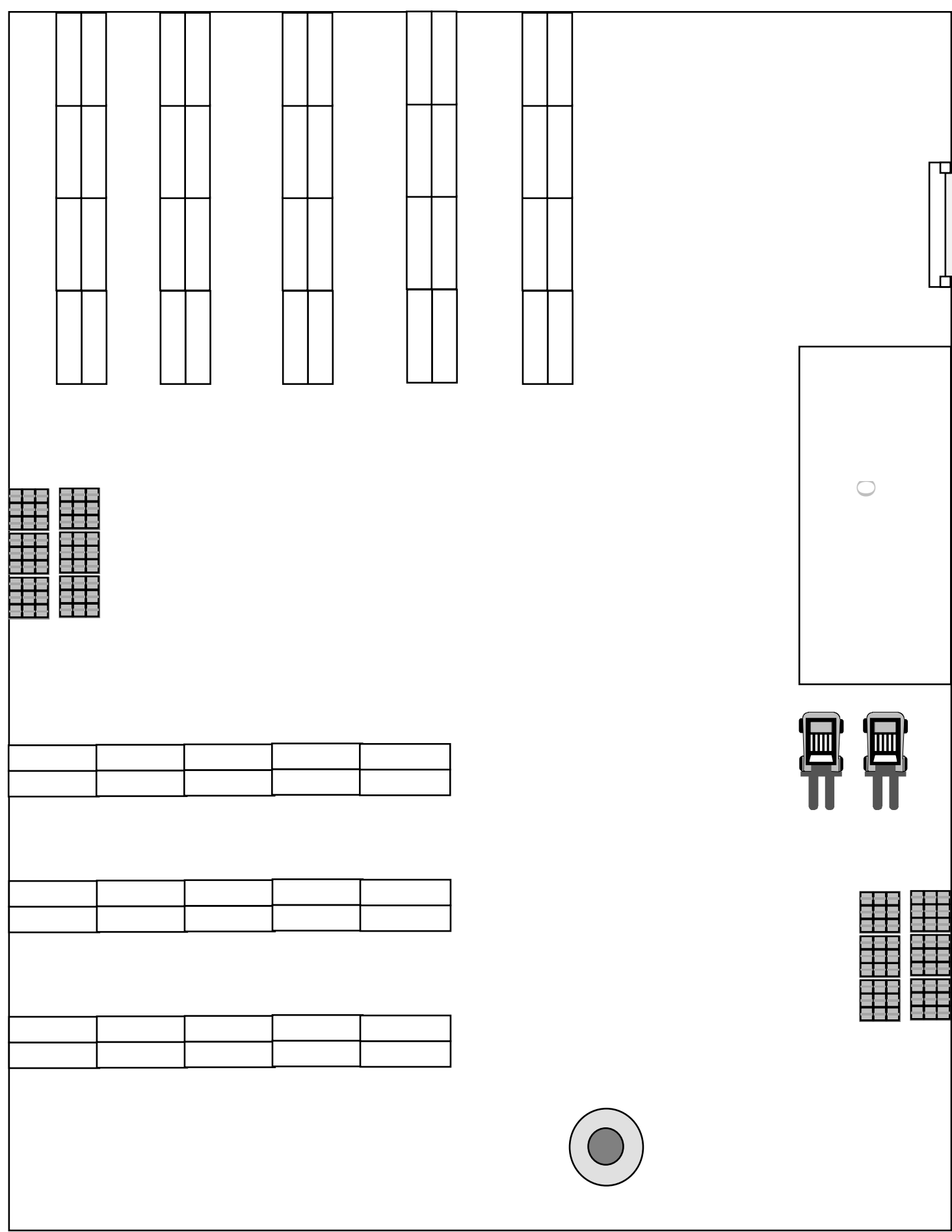

Figure 48. Homestead Warehouse $1800 \mathrm{ft} 2$ 


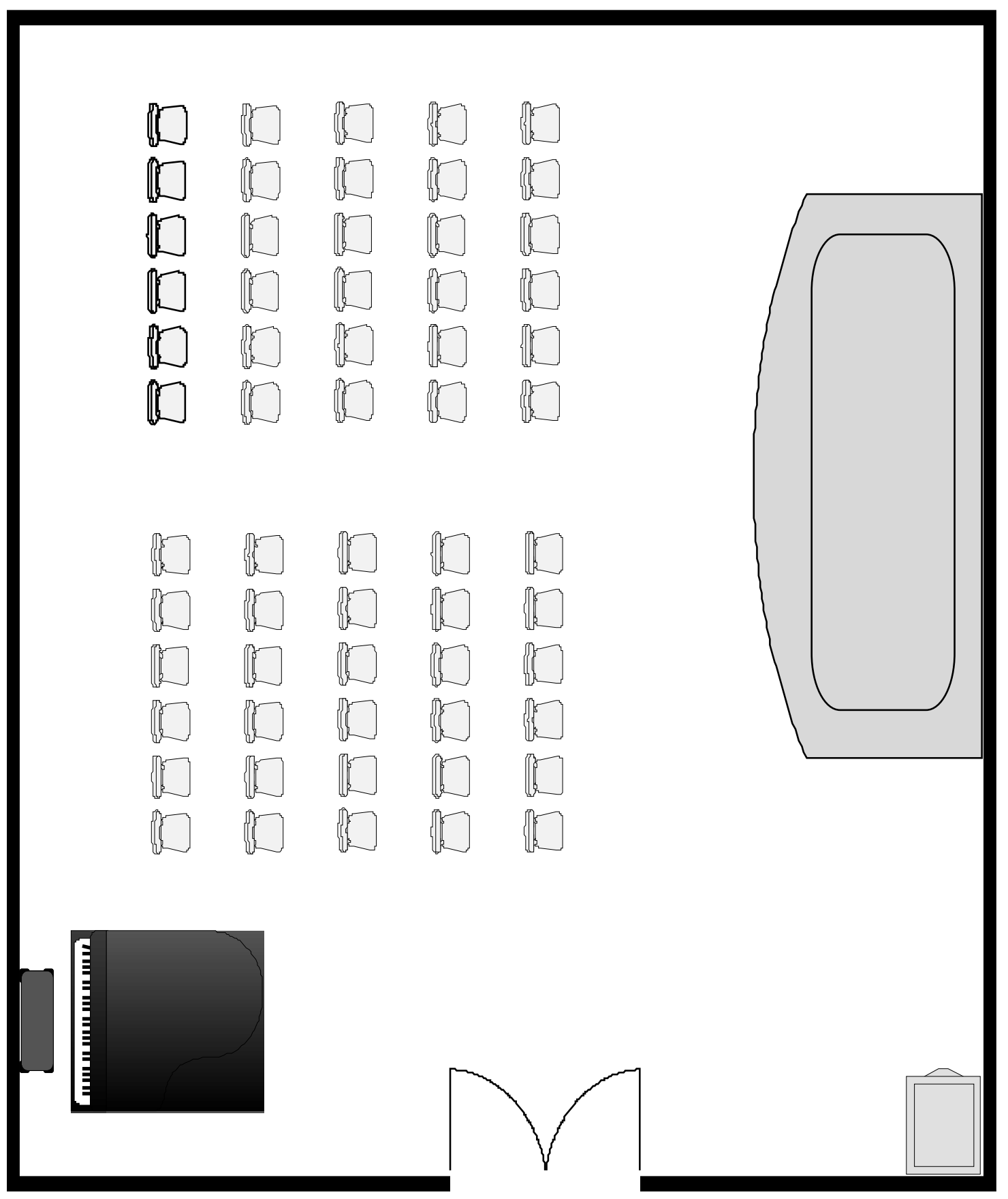

Figure 49. Westwood Christian School $600 \mathrm{ft} 2$ 

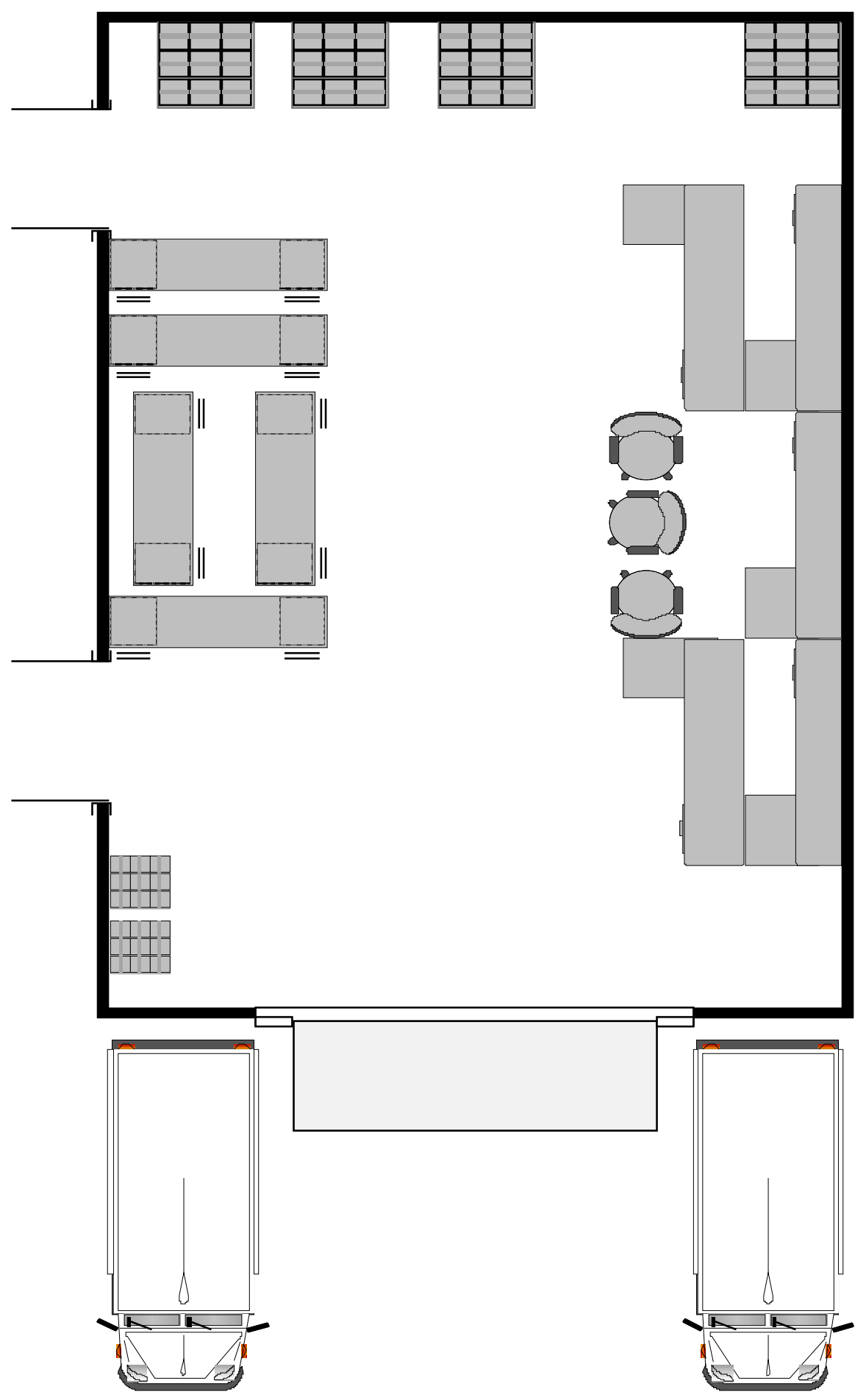

Figure 50. Schoolboard Warehouse 


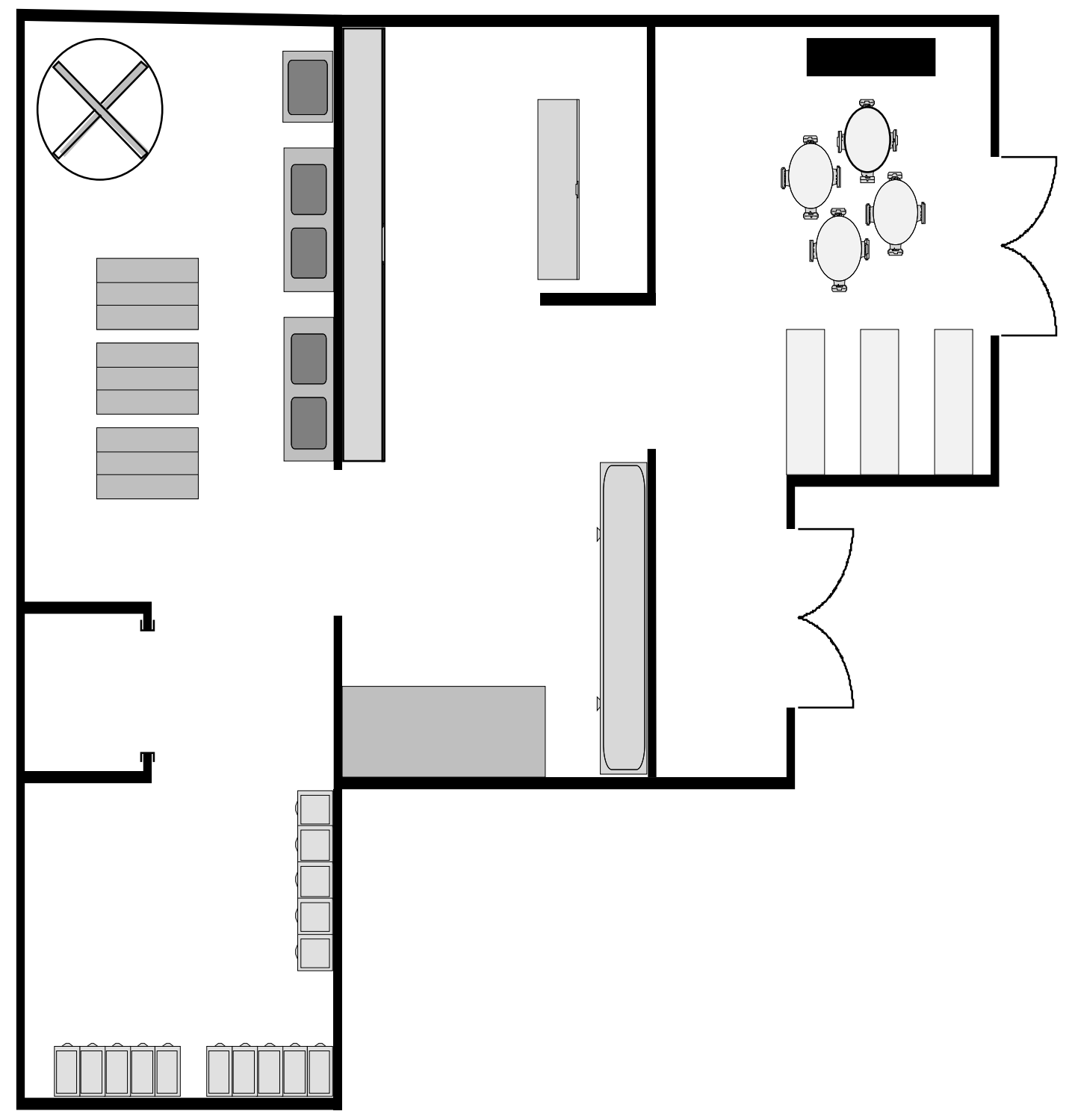

Figure 51. Kendall Warehouse II 
Table 36. Canine rooster and trial schedule

\begin{tabular}{lllll}
\hline FIU Number & Canine Name & Handler & Date & Location \\
\hline 1 & Leo & Nunn & $9 / 15 / 2014$ & Camp Pendleton, CA \\
2 & Denny & Goddard & $9 / 15 / 2014$ & Camp Pendleton, CA \\
3 & Gus & Gardea & $9 / 15 / 2014$ & Camp Pendleton, CA \\
4 & Zidane & Nunn & $9 / 15 / 2014$ & Camp Pendleton, CA \\
5 & Maika & Dunaway & $9 / 15 / 2014$ & Camp Pendleton, CA \\
6 & Red & Crisp & $9 / 15 / 2014$ & Camp Pendleton, CA \\
7 & Freddie & Kosiara & $9 / 15 / 2014$ & Camp Pendleton, CA \\
8 & Lotor & Nunn & $9 / 15 / 2014$ & Camp Pendleton, CA \\
9 & Linda & Gann & $9 / 15 / 2014$ & Camp Pendleton, CA \\
10 & Astor & Kosiara & $9 / 15 / 2014$ & Camp Pendleton, CA \\
11 & Vicky & Shahan & $9 / 15 / 2014$ & Camp Pendleton, CA \\
12 & Largo & Wagman & $9 / 15 / 2014$ & Camp Pendleton, CA
\end{tabular}


Table 37. Canine ORTs Water Gel Aged Dynamic Collected Samples $(n=12)$

\begin{tabular}{cccc}
\hline Canine & Control & WG & Blank \\
\hline 1 & A & I & N \\
2 & A & N & N \\
3 & A & A & N \\
4 & A & I & N \\
5 & A & N & A \\
6 & A & A & N \\
7 & A & I & N \\
8 & A & N & N \\
9 & A & N & N \\
10 & A & A & I \\
11 & A & N & N \\
12 & A & I & N
\end{tabular}

Table 38. Canine ORTs Water Gel Fresh Dynamic Collected Samples (n=12)

\begin{tabular}{cccc}
\hline Canine & Control & WG & Blank \\
\hline 1 & A & A & A \\
2 & A & N & A \\
3 & A & A & N \\
5 & A & A & A \\
6 & A & N & N \\
7 & A & A & N \\
8 & A & A & N \\
9 & A & I & A \\
10 & A & N & A \\
11 & A & A & A \\
12 & A & A & N \\
\hline
\end{tabular}


VITA

\section{ADHLY MARIE HUERTAS RIVERA}

Born, Bayamón, Puerto Rico

2006-2011

B.Sc, Chemistry

University of Puerto Rico

San Juan, PR

(Cum Laude)

2011-2012

Teaching Assistant

Florida International University

Miami, FL

2012-2014

Research Assistant

Florida International University

Miami, FL

2014-2016

Teaching Assistant

Florida International University

Miami, FL

2016

Doctoral Evidence Acquisition Fellowship

Florida International University

Miami, FL

\section{PUBLICATIONS}

Sanchez CL, Huertas AM, Prada PA, Furton KG (2016) A Non-Contact Passive Approach for the Effective Collection of Target Explosive Volatiles for Canine Training Aid Development. J Forensic Sci Criminol 4(2): 205

Huertas AM, Sanchez CL, Prada PA, Furton KG (2016) Transfer Validation and Evaluation of Storage Containment System for Dynamically Collected Explosive Samples. In Preparation

Huertas AM, Furton KG (2016) Evaluation of the Volatile Organic Compounds from an Illicit Substance for the Development of Improved Field Calibrants for Instrumental and Canine Detection. In Preparation 


\section{PRESENTATIONS}

Huertas, Adhly, B.Sc and Furton Kenneth G, Ph.D. (April 6, 2016) Illicit Substance Volatile Organic Compounds (VOCs) Analysis for Canine Detection. Poster session presented at: GSAW Scholarly Forum, Florida International University, Miami, FL.

Huertas, Adhly, B.Sc and Furton Kenneth G, Ph.D. (February 19, 2015) Illicit Substance Volatile Organic Compounds (VOCs) Analysis for Canine Detection. Poster session presented at: AAFS 67th Annual Scientific Meeting, Hyatt Regency Orlando, Orlando, FL.

Huertas, Adhly, B.Sc, Sanchez, Claudia L., B.A., and Furton Kenneth G, Ph.D. (May 8, 2014) Evaluation of the Volatile Organic Compounds from an illicit substance for the Development of Improved Field Calibrants for Instrumental and Canine Detection. Poster session presented at: Florida Annual Meeting and Exposition, Innisbrook, A Salamander ${ }^{\circledR}$ Golf \& Spa Resort, Palm Harbor, FL

Huertas, Adhly, B.Sc, Sanchez, Claudia L., B.A., and Furton Kenneth G, Ph.D. (February $21,2014)$ Evaluation of the Volatile Organic Compounds from an illicit substance for the Development of Improved Field Calibrants for Instrumental and Canine Detection. AAFS 66th Annual Scientific Meeting, Washington State Convention Center, Seattle, WA 